\title{
DFT-Based Stereochemical Rationales for the Bifunctional Brønsted Acid/Base-Catalyzed Diastereodivergent and Enantioselective aza-Henry Reactions of $\alpha$-Nitro Esters
}

\author{
Thomas J. Struble,${ }^{1 \dagger}$ Ivor Smajlagic,${ }^{2 \dagger}$ Hayden Foy,${ }^{2 \dagger}$ Travis Dudding, ${ }^{2 *}$ and Jeffrey N.
} Johnston $^{1 *}$

${ }^{1}$ Department of Chemistry and Vanderbilt Institute of Chemical Biology, Vanderbilt University, Nashville, Tennessee 37235

${ }^{2}$ Department of Chemistry, Brock University, 1812 Sir Isaac Brock Way, St. Catharines, ON L2S 3A1, Canada

*Email: tdudding@brocku.ca

*Email: jeffrey.n.johnston@vanderbilt.edu

\section{SUPPORTING INFORMATION}

Computational Methods S2

Cartesian Coordinates and Energies of Calculated Structures S3

Optimized Truncated Models, syn- and anti-Products, and Transition State Structures Using the $\mathrm{C}_{1}$ -

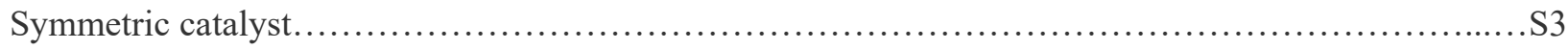

Optimized Precomplex Structures Using the $\mathrm{C}_{1}$-Symmetric Catalyst................................ 72

Parallel Investigation of $C_{2}$-Symmetric PBAM-Catalyzed anti-Selective aza-Henry Reactions ...........S1 15

Optimized Transition State- and Precomplex Structures Using the $\mathrm{C}_{2}$-Symmetric catalyst.............S116

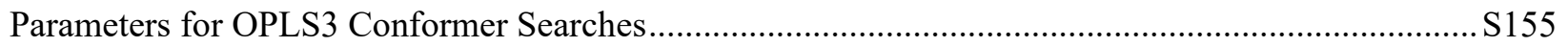

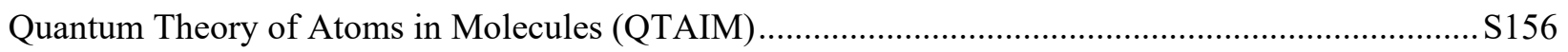

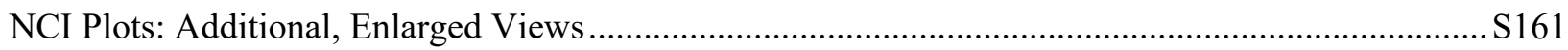

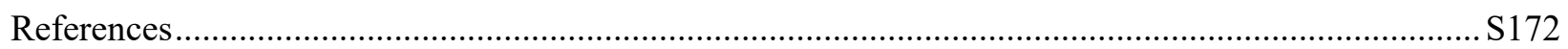




\section{Computational Methods}

Density functional theory (DFT) calculations were performed using Gaussian $09^{1}$ and all geometry optimizations employed the M06-2X $\mathrm{X}^{2}$ functional with a 6-31G(d) basis set. This approach was ultimately arrived at upon screening multiple methods, including the use of the B3LYP and B3LYP-D3 functionals, which includes Becke's three-parameter gradient-corrected exchange functional ${ }^{3}$ and the LYP correlation functional ${ }^{4}$ of Lee, Yang and Parr, as well as Grimme's dispersion correction method $^{5}$ for the latter utilizing a 6-31G(d) basis set. Moreover, the M06-2X functional was found to be optimal and has been proven to be superior to B3LYP in terms of providing accurate model systems taking dispersion and ionic hydrogen-bonding interactions into account. Given the large size of these structures, however, it was not feasible to use a more complex basis set, as the computational demand was too large. The optimized geometries were verified as either transition state structures (one imaginary frequency) or minima (zero imaginary frequencies) by frequency calculations. Intrinsic reaction coordinate (IRC) calculations were performed to confirm that all transition state structures were linked to relevant minima. Notably, numerous transition state geometries and addition modes were considered, from which the lowest energy models were included. The energies obtained from the lowest-energy optimized structures at the M06-2X/6-31G(d) level of theory were further refined by single-point calculations performed at the M06-2X/6-311++G(2d,2p) ${ }^{6}$ level of theory using the solvation model density (SMD) continuum solvation model with the default parameters of toluene $(\mathrm{e}=2.38)$ to account for solvent. ${ }^{7.8}$ The thermal corrections to the Gibbs free energies (temperature $=298.15 \mathrm{~K}$, pressure $=$ $1 \mathrm{~atm})$ computed at the lower level of theory (M06-2X 6-31G(d)) were added to the electronic energies obtained from the single-point calculations to provide the reported Gibbs free energies. The key word integral=grid=ultrafine was used for all calculations. The reported non-covalent interaction ( $\mathrm{NCI}$ ) visualizations (isovalue $=0.3, \min =-0.03$ and $\max =0.07$ ) were generated with Schrödinger Jaguar. ${ }^{9}$ The 3D images of all optimized geometries were generated with CYLview. ${ }^{10}$ GaussView5 ${ }^{11}$ was used to construct all structures prior to optimization and to visualize the output from the Gaussian 09 calculations. Monte Carlo conformational searches (MCCS) with an OPLS3 ${ }^{12}$ force field were performed on the full catalyst systems using the Schrödinger Macromodel program. ${ }^{13}$ 


\section{Cartesian Coordinates and Energies of Calculated Structures}

\section{Optimized Truncated Models, syn- and anti-Products, and Transition State Structures Using the $\mathrm{C}_{1-S y m m e t r i c}$ catalyst.}

Table S1. Single point energies of all structures calculated at the $\operatorname{SMD}_{(\text {Toluene) }} M 06-2 X / 6-311++G(2 d, 2 p)$ level of theory and thermal correction to Gibbs Free Energy and Gibbs Free Energy calculated at the M06-2X/6-31G(d) level of theory. All energies are reported in Hartrees.

\begin{tabular}{|c|c|c|c|c|}
\hline Structure & $\begin{array}{c}\text { Single Point } \\
\text { Energies (E) } \\
\text { SMD }_{\text {(Toluene) M06- }} \\
\text { 2X/6- } \\
\text { 311++G(2d,2p) }\end{array}$ & $\begin{array}{l}\text { Thermal } \\
\text { Corrections } \\
\text { to Gibbs } \\
\text { Free } \\
\text { Energies (G) } \\
\text { M06-2X/6- } \\
\text { 31G(d) }\end{array}$ & $\begin{array}{c}\text { Gibbs Free } \\
\text { Energies (G) } \\
\text { M06-2X/6- } \\
\text { 31G(d) }\end{array}$ & $\begin{array}{c}\text { Gibbs Free } \\
\text { Energies (G) } \\
\text { SMD }_{\text {(Toluene)M06- }} \text { 2X/6- } \\
\text { 2X/6- } \\
\text { 311++G(2d,2p)// } \\
\text { M06-2X/6-31G(d) }\end{array}$ \\
\hline 10 & -881.628493 & 0.280415 & -881.027153 & -881.348078 \\
\hline 11 & -939.234160 & 0.284863 & -938.582963 & -938.949297 \\
\hline 12 & -1820.935106 & 0.593049 & -1819.741323 & -1820.342057 \\
\hline 13 & -1820.939177 & 0.587702 & -1819.752635 & -1820.351475 \\
\hline 14 & -1820.947633 & 0.586816 & -1819.759489 & -1820.360817 \\
\hline 15 & -496.983637 & 0.161812 & -496.612472 & -496.821825 \\
\hline 16 & -1436.291344 & 0.472254 & -1435.330841 & -1435.81909 \\
\hline 17 & -1436.301503 & 0.468886 & -1435.345514 & -1435.832617 \\
\hline 18 & -1436.301520 & 0.466523 & -1435.346346 & -1435.834997 \\
\hline 19 & -1553.122742 & 0.517458 & -1552.080668 & -1552.605014 \\
\hline 20 & -1553.122348 & 0.518435 & -1552.077399 & -1552.603913 \\
\hline syn-product & -1493.305096 & 0.456508 & -1492.344264 & -1492.848588 \\
\hline $\begin{array}{c}\text { anti- } \\
\text { product }\end{array}$ & -1493.302498 & 0.454949 & -1492.340087 & -1492.847549 \\
\hline $\begin{array}{c}\text { anti- } \\
(2 R, 3 R)-\mathrm{TS}\end{array}$ & -3258.781514 & 1.117935 & -3256.586769 & -3257.663579 \\
\hline $\begin{array}{c}s y n-(2 S, 3 R)- \\
\text { TS }\end{array}$ & -3258.790148 & 1.123332 & -3256.597713 & -3257.666816 \\
\hline $\begin{array}{c}s y n-(2 R, 3 S)- \\
\text { TS }\end{array}$ & -3258.785444 & 1.120363 & -3256.591507 & -3257.665081 \\
\hline $\begin{array}{c}\text { anti- } \\
(2 S, 3 S)-\mathrm{TS}\end{array}$ & -3258.788448 & 1.122464 & -3256.597146 & -3257.665984 \\
\hline $\begin{array}{c}\text { anti- } \\
(2 R, 3 R)- \\
\text { TS2 }\end{array}$ & -3258.778203 & 1.120112 & -3256.583866 & -3257.658091 \\
\hline $\begin{array}{c}s y n-(2 R, 3 S)- \\
\text { TS2 }\end{array}$ & -3258.785755 & 1.121053 & -3256.589032 & -3257.664702 \\
\hline
\end{tabular}




\begin{tabular}{|c|c|c|c|c|}
\hline $\begin{array}{c}\text { anti- } \\
(\mathbf{2 S , 3 S ) - T S 2}\end{array}$ & -3258.781579 & 1.120286 & -3256.584495 & -3257.661293 \\
\hline $\begin{array}{c}\text { syn-(2S,3R)- } \\
\text { TS2 }\end{array}$ & -3258.785871 & 1.123500 & -3256.590956 & -3257.662371 \\
\hline
\end{tabular}

Structure 10

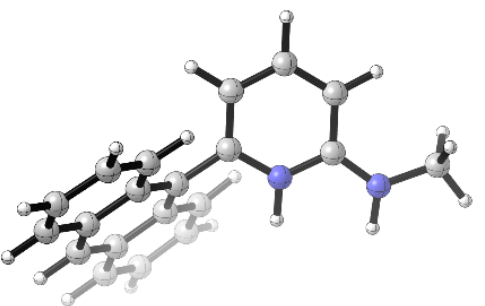

- Thermochemistry -

Zero-point correction=

0.325639 (Hartree/Particle)

Thermal correction to Energy=

0.342937

Thermal correction to Enthalpy=

0.343881

Thermal correction to Gibbs Free Energy=

0.280415

Sum of electronic and zero-point Energies=

$-880.981929$

Sum of electronic and thermal Energies=

$-880.964631$

Sum of electronic and thermal Enthalpies=

$-880.963687$

Sum of electronic and thermal Free Energies=

$-881.027153$

Number of Imaginary Frequencies $=0$

E (Single Point Energy) $\left[\mathrm{SMD}_{\text {(Toluene) }} \mathrm{M} 06-2 \mathrm{X} / 6-311++\mathrm{G}(2 \mathrm{~d}, 2 \mathrm{p})\right]=-881.628493$

$\begin{array}{lrrr}\mathrm{C} & 0.90349500 & -0.46787500 & 0.50413700 \\ \mathrm{C} & 1.35095500 & -1.20983600 & 1.57049300 \\ \mathrm{C} & 2.72686300 & -1.47639200 & 1.68695500 \\ \mathrm{C} & 3.63520800 & -1.01087100 & 0.76232600 \\ \mathrm{H} & 3.08681300 & -2.05635700 & 2.53085300 \\ \mathrm{C} & 3.16963500 & -0.24871400 & -0.32943200 \\ \mathrm{H} & 4.69314100 & -1.21541300 & 0.85779400\end{array}$




$\begin{array}{lrrr}\mathrm{N} & 1.83197400 & -0.02399900 & -0.40406300 \\ \mathrm{H} & 1.46208900 & 0.52588400 & -1.17690900 \\ \mathrm{~N} & 3.96334500 & 0.25621400 & -1.28244700 \\ \mathrm{H} & 3.54863600 & 0.77578600 & -2.04385000 \\ \mathrm{C} & 5.40827800 & 0.06141200 & -1.29887600 \\ \mathrm{H} & 5.80796000 & 0.57005900 & -2.17434900 \\ \mathrm{H} & 5.86787800 & 0.49242300 & -0.40513400 \\ \mathrm{H} & 5.65781400 & -1.00123600 & -1.36750400 \\ \mathrm{H} & 0.63727300 & -1.56115600 & 2.30506300 \\ \mathrm{C} & -0.49843100 & -0.08757000 & 0.23736900 \\ \mathrm{C} & -0.83663000 & 1.28466500 & 0.21244200 \\ \mathrm{C} & -1.45871300 & -1.09531900 & -0.00047200 \\ \mathrm{C} & 0.08757000 & 2.33499000 & 0.52436300 \\ \mathrm{C} & -2.18835000 & 1.65460400 & -0.09575400 \\ \mathrm{C} & -2.81103000 & -0.69909200 & -0.27914000 \\ \mathrm{C} & -1.15645400 & -2.49443100 & -0.01780700 \\ \mathrm{C} & -0.29708700 & 3.64437600 & 0.48026300 \\ \mathrm{H} & 1.09687300 & 2.09051100 & 0.84226200 \\ \mathrm{C} & -2.54639100 & 3.03820600 & -0.13969700 \\ \mathrm{C} & -3.13483400 & 0.65656300 & -0.32895500 \\ \mathrm{C} & -3.80279500 & -1.70112900 & -0.51900300 \\ \mathrm{H} & -0.13781600 & -2.82706300 & 0.14984200 \\ \mathrm{C} & -2.13228800 & -3.41910500 & -0.25420300 \\ \mathrm{C} & -1.62853700 & 4.00846900 & 0.12852800 \\ \mathrm{H} & 0.41606700 & 4.42221800 & 0.73412500 \\ \mathrm{H} & -3.57318600 & 3.29458600 & -0.38417200 \\ \mathrm{H} & -4.15933100 & 0.94494200 & -0.55341000 \\ \mathrm{C} & -3.47864400 & -3.02365800 & -0.50070700 \\ \mathrm{H} & -4.82029400 & -1.37825500 & -0.71928200 \\ \mathrm{H} & -1.87987300 & -4.47459400 & -0.26152000 \\ \mathrm{H} & -1.90741200 & 5.05597700 & 0.09711700 \\ \mathrm{H} & -4.23479000 & -3.77957100 & -0.68233200\end{array}$

\section{Structure 11}

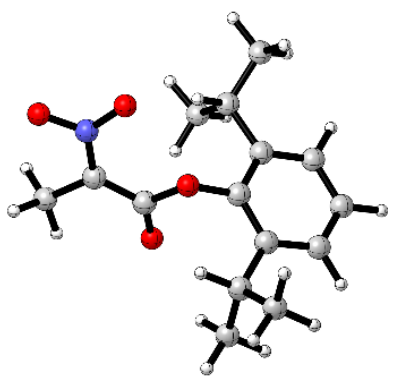

- Thermochemistry - 


$\begin{array}{ll}\text { Zero-point correction }= & 0.334608 \text { (Hartree/Particle) } \\ \text { Thermal correction to Energy= } & 0.355202 \\ \text { Thermal correction to Enthalpy= } & 0.356146 \\ \text { Thermal correction to Gibbs Free Energy= } & 0.284863 \\ \text { Sum of electronic and zero-point Energies }= & -938.533219 \\ \text { Sum of electronic and thermal Energies }= & -938.512624 \\ \text { Sum of electronic and thermal Enthalpies }= & -938.511680 \\ \text { Sum of electronic and thermal Free Energies= } & -938.582963\end{array}$

Number of Imaginary Frequencies $=0$

E (Single Point Energy) $\left[\mathrm{SMD}_{(\text {Toluene) }} \mathrm{M} 06-2 \mathrm{X} / 6-311++\mathrm{G}(2 \mathrm{~d}, 2 \mathrm{p})\right]=-939.234160$

$\begin{array}{lrrr}\mathrm{C} & 1.01214100 & -0.88177900 & 0.50644800 \\ \mathrm{O} & 0.42135600 & -1.17513900 & 1.54487900 \\ \mathrm{O} & 0.27847100 & -0.35540800 & -0.55378500 \\ \mathrm{C} & -0.90789500 & 0.26072200 & -0.23548700 \\ \mathrm{C} & -0.87719000 & 1.64905800 & -0.03118600 \\ \mathrm{C} & -2.09684800 & -0.47228200 & -0.19779700 \\ \mathrm{C} & -2.08568400 & 2.30323000 & 0.20284100 \\ \mathrm{C} & -3.28577200 & 0.22184600 & 0.04226700 \\ \mathrm{C} & -3.28620500 & 1.59750200 & 0.23760200 \\ \mathrm{H} & -2.09020000 & 3.37867800 & 0.36122000 \\ \mathrm{H} & -4.22351200 & -0.32864000 & 0.08344800 \\ \mathrm{H} & -4.22055200 & 2.12150900 & 0.42080100 \\ \mathrm{C} & 2.39915500 & -1.06180300 & 0.27452900 \\ \mathrm{C} & 3.21115300 & -1.80293300 & 1.29079700 \\ \mathrm{H} & 4.01362100 & -1.17845500 & 1.70223500 \\ \mathrm{H} & 2.55205800 & -2.11778500 & 2.10135400 \\ \mathrm{H} & 3.69848300 & -2.68348000 & 0.85528000 \\ \mathrm{~N} & 3.10104200 & -0.55648700 & -0.80897000 \\ \mathrm{O} & 4.31867200 & -0.83244100 & -0.86648300 \\ \mathrm{O} & 2.57325200 & 0.15544000 & -1.68422600 \\ \mathrm{C} & -2.08875900 & -1.97702600 & -0.37984000 \\ \mathrm{H} & -1.07352900 & -2.26071500 & -0.67127300 \\ \mathrm{C} & -3.04702600 & -2.42215200 & -1.48943200 \\ \mathrm{H} & -2.81611300 & -1.92240400 & -2.43511200\end{array}$




$\begin{array}{lrrr}\mathrm{H} & -2.97542200 & -3.50464800 & -1.64428000 \\ \mathrm{H} & -4.08819200 & -2.19392000 & -1.23196600 \\ \mathrm{C} & -2.41042400 & -2.67574800 & 0.94575200 \\ \mathrm{H} & -1.68000500 & -2.37485400 & 1.70023200 \\ \mathrm{H} & -3.41667700 & -2.40612500 & 1.29090400 \\ \mathrm{H} & -2.37515400 & -3.76523100 & 0.82567400 \\ \mathrm{C} & 0.46360700 & 2.35814400 & -0.00774500 \\ \mathrm{H} & 1.15141100 & 1.77233200 & -0.62909200 \\ \mathrm{C} & 0.40189100 & 3.78192600 & -0.56256900 \\ \mathrm{H} & 1.41228100 & 4.19849900 & -0.62940700 \\ \mathrm{H} & -0.04292200 & 3.80182800 & -1.56260900 \\ \mathrm{H} & -0.18313400 & 4.44673400 & 0.08506200 \\ \mathrm{C} & 1.01143700 & 2.36450200 & 1.42837600 \\ \mathrm{H} & 1.03340400 & 1.35460400 & 1.84767400 \\ \mathrm{H} & 2.02753400 & 2.77389400 & 1.45008000 \\ \mathrm{H} & 0.37383000 & 2.98473700 & 2.07213900\end{array}$

Structure 12

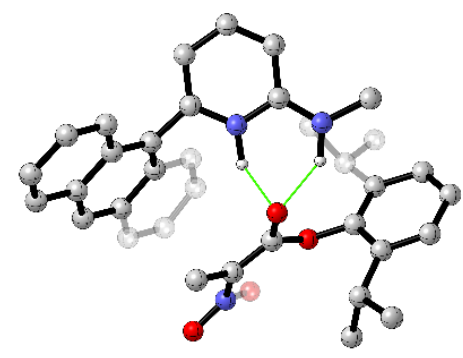

- Thermochemistry -

Zero-point correction=

Thermal correction to Energy=

Thermal correction to Enthalpy=

Thermal correction to Gibbs Free Energy=

Sum of electronic and zero-point Energies $=$

Sum of electronic and thermal Energies=

Sum of electronic and thermal Enthalpies=

Sum of electronic and thermal Free Energies=
0.662501 (Hartree/Particle)

0.700773

0.701717

0.593049

$-1819.671872$

$-1819.633600$

$-1819.632656$

$-1819.741323$

Number of Imaginary Frequencies $=0$ 
E (Single Point Energy) $\left[\mathrm{SMD}_{\text {(Toluene) }} \mathrm{M} 06-2 \mathrm{X} / 6-311++\mathrm{G}(2 \mathrm{~d}, 2 \mathrm{p})\right]=-1820.935106$

$\begin{array}{lrrr}\mathrm{C} & 2.09388300 & 2.06596100 & 0.26814000 \\ \mathrm{C} & 2.49867700 & 3.31451700 & 0.68248200 \\ \mathrm{C} & 1.59621000 & 4.38830600 & 0.55799400 \\ \mathrm{C} & 0.33317900 & 4.21636800 & 0.04099000 \\ \mathrm{H} & 1.89834700 & 5.37630900 & 0.89196600 \\ \mathrm{C} & -0.05823500 & 2.92395800 & -0.38792200 \\ \mathrm{H} & -0.36375600 & 5.04038900 & -0.04024900 \\ \mathrm{~N} & 0.85119600 & 1.92181300 & -0.26173500 \\ \mathrm{H} & 0.51258900 & 0.96784000 & -0.57259100 \\ \mathrm{~N} & -1.24856200 & 2.61318000 & -0.90226000 \\ \mathrm{H} & -1.42017400 & 1.61484400 & -1.07936000 \\ \mathrm{C} & -2.34551800 & 3.55399900 & -0.99168300 \\ \mathrm{H} & -3.20156600 & 3.02461800 & -1.41027100 \\ \mathrm{H} & -2.62929000 & 3.93615400 & -0.00340400 \\ \mathrm{H} & -2.09060400 & 4.39603400 & -1.64411200 \\ \mathrm{C} & -0.88222700 & -1.09568700 & -0.38617500 \\ \mathrm{O} & -0.63745300 & -0.05397300 & -1.07115500 \\ \mathrm{O} & -2.05549600 & -1.12646300 & 0.32116000 \\ \mathrm{C} & -3.10121300 & -0.32673900 & -0.10386600 \\ \mathrm{C} & -3.64223300 & -0.48162600 & -1.39251100 \\ \mathrm{C} & -3.65819900 & 0.53228000 & 0.85211600 \\ \mathrm{C} & -4.72834600 & 0.33259400 & -1.72524500 \\ \mathrm{C} & -4.75696400 & 1.30839500 & 0.47763000 \\ \mathrm{C} & -5.28060500 & 1.22233200 & -0.80704200 \\ \mathrm{H} & -5.16997600 & 0.25239300 & -2.71267900 \\ \mathrm{H} & -5.21425700 & 1.97611600 & 1.20155000 \\ \mathrm{H} & -6.13634200 & 1.82891900 & -1.08848500 \\ \mathrm{C} & -0.03348400 & -2.19288600 & -0.37448600 \\ \mathrm{C} & 1.12746200 & -2.24022500 & -1.32386200 \\ \mathrm{H} & 0.82640000 & -2.58889300 & -2.32023300 \\ \mathrm{H} & 1.56564400 & -1.24450300 & -1.44180100 \\ \mathrm{H} & -1.89067300 & -2.92219700 & -0.95046600 \\ \mathrm{H} & -0.25713100 & -3.32066000 & 0.43893600 \\ \mathrm{~N} & -1.10538800 & -3.28766100 & 1.33721700 \\ \mathrm{O} & 0.45749300 & -4.31207100 & 0.25468200 \\ \mathrm{C} & -3.04749000 & 0.59404600 & 2.23972400 \\ \mathrm{H} & -2.71998600 & -0.42184500 & 2.48845600 \\ \mathrm{H} & -4.02096700 & 1.06835900 & 3.31757300 \\ \mathrm{H} & -3.56049600 & 0.96748000 & 4.30507600 \\ \mathrm{H} & -1.80148900 & 1.48361400 & 2.20376800 \\ \mathrm{H} & & 1.12033800 & 1.43852700\end{array}$




$\begin{array}{lrrr}\mathrm{H} & -2.07504900 & 2.51940400 & 1.96196100 \\ \mathrm{H} & -1.28566600 & 1.48389300 & 3.17162600 \\ \mathrm{C} & -3.11334000 & -1.55567500 & -2.33241000 \\ \mathrm{H} & -2.03132500 & -1.41670900 & -2.42928900 \\ \mathrm{C} & -3.36926300 & -2.95312900 & -1.74237500 \\ \mathrm{H} & -2.93284300 & -3.71810500 & -2.39269100 \\ \mathrm{H} & -2.93759600 & -3.06554300 & -0.74440300 \\ \mathrm{H} & -4.44748100 & -3.13750600 & -1.67495300 \\ \mathrm{C} & -3.69984200 & -1.47080400 & -3.74162700 \\ \mathrm{H} & -3.53757200 & -0.48763700 & -4.19526700 \\ \mathrm{H} & -3.22251300 & -2.21976900 & -4.37993800 \\ \mathrm{H} & -4.77601900 & -1.67776000 & -3.74091800 \\ \mathrm{H} & 3.48356500 & 3.44187900 & 1.11342900 \\ \mathrm{C} & 2.88482500 & 0.81324000 & 0.37162700 \\ \mathrm{C} & 4.03034400 & 0.62712900 & -0.42638900 \\ \mathrm{C} & 2.40290200 & -0.20899600 & 1.21486200 \\ \mathrm{C} & 4.54670600 & 1.62837100 & -1.31092300 \\ \mathrm{C} & 4.70032500 & -0.64277800 & -0.38004200 \\ \mathrm{C} & 3.04945700 & -1.48822400 & 1.20472000 \\ \mathrm{C} & 1.28539400 & -0.02871200 & 2.09005600 \\ \mathrm{C} & 5.66280400 & 1.39509900 & -2.05981400 \\ \mathrm{H} & 4.03685400 & 2.58342300 & -1.38499100 \\ \mathrm{C} & 5.86729900 & -0.84350200 & -1.18203700 \\ \mathrm{C} & 4.18443700 & -1.66767600 & 0.41530000 \\ \mathrm{C} & 2.48760800 & -2.55864800 & 1.97038000 \\ \mathrm{H} & 0.84223400 & 0.95590200 & 2.18212400 \\ \mathrm{C} & 0.78102800 & -1.07268100 & 2.80865900 \\ \mathrm{C} & 6.34115400 & 0.14327000 & -1.99285700 \\ \mathrm{H} & 6.03830100 & 2.16899600 & -2.72207400 \\ \mathrm{H} & 6.36310500 & -1.80901300 & -1.13074300 \\ \mathrm{H} & 4.67256500 & -2.64006000 & 0.40610500 \\ \mathrm{C} & 1.37660600 & -2.36575800 & 2.73488500 \\ \mathrm{H} & 2.94739900 & -3.53993100 & 1.89945600 \\ \mathrm{H} & -0.09003600 & -0.92436100 & 3.44008900 \\ \mathrm{H} & 7.22688900 & -0.01968200 & -2.59810400 \\ \mathrm{H} & 0.92024800 & -3.19625300 & 3.26139400\end{array}$




\section{Structure 13}

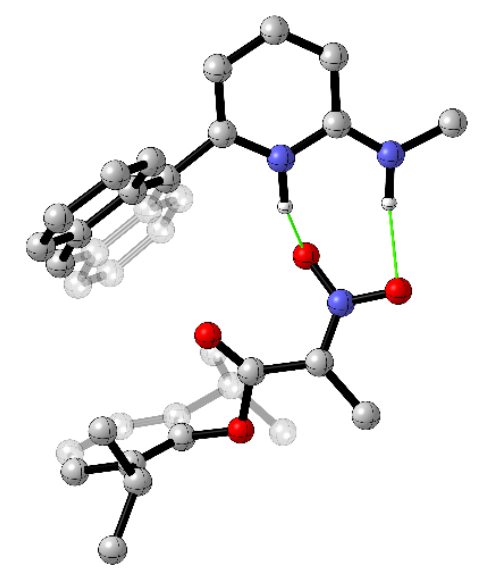

- Thermochemistry -

Zero-point correction $=$

0.660156 (Hartree/Particle)

Thermal correction to Energy=

0.699173

Thermal correction to Enthalpy=

0.700117

Thermal correction to Gibbs Free Energy=

0.587702

Sum of electronic and zero-point Energies $=$

$-1819.680181$

Sum of electronic and thermal Energies=

$-1819.641165$

Sum of electronic and thermal Enthalpies=

$-1819.640220$

Sum of electronic and thermal Free Energies=

$-1819.752635$

Number of Imaginary Frequencies $=0$

E (Single Point Energy) $\left[\mathrm{SMD}_{\text {(Toluene) }} \mathrm{M} 06-2 \mathrm{X} / 6-311++\mathrm{G}(2 \mathrm{~d}, 2 \mathrm{p})\right]=-1820.939177$

$\begin{array}{lrrr}\mathrm{C} & 3.44141900 & 0.21470700 & -0.75597400 \\ \mathrm{C} & 4.69592000 & 0.43219700 & -1.28578500 \\ \mathrm{C} & 5.66683200 & -0.57561600 & -1.15633800 \\ \mathrm{C} & 5.39160200 & -1.75817600 & -0.51166300 \\ \mathrm{H} & 6.65648700 & -0.41905400 & -1.57503000 \\ \mathrm{C} & 4.09272900 & -1.96068900 & 0.02085600 \\ \mathrm{H} & 6.13865200 & -2.53453400 & -0.41102000 \\ \mathrm{~N} & 3.18300500 & -0.96142100 & -0.13086900 \\ \mathrm{~N} & 3.71162100 & -3.05953800 & 0.67046600 \\ \mathrm{H} & 2.74680300 & -3.13024400 & 1.03586800 \\ \mathrm{C} & 4.59937200 & -4.17643100 & 0.90606100\end{array}$




$\begin{array}{lrrr}\mathrm{H} & 4.04326300 & -4.92220100 & 1.47343400 \\ \mathrm{H} & 4.93668200 & -4.63070100 & -0.03282400 \\ \mathrm{H} & 5.47710600 & -3.87740000 & 1.49056900 \\ \mathrm{C} & -1.33535600 & -0.99491200 & 1.09748500 \\ \mathrm{O} & -0.84743600 & -0.15189800 & 0.38738400 \\ \mathrm{O} & -2.69856600 & -1.04907400 & 1.30332600 \\ \mathrm{C} & -3.45732000 & -0.31125800 & 0.39751600 \\ \mathrm{C} & -3.81065400 & -0.94666600 & -0.80123800 \\ \mathrm{C} & -3.83555400 & 0.99128100 & 0.71384900 \\ \mathrm{C} & -4.62544000 & -0.23921000 & -1.68361400 \\ \mathrm{C} & -4.63964700 & 1.66837000 & -0.20923700 \\ \mathrm{C} & -5.03923000 & 1.05923900 & -1.39111200 \\ \mathrm{H} & -4.92948400 & -0.69640900 & -2.62020900 \\ \mathrm{H} & -4.95135400 & 2.68769300 & 0.00352200 \\ \mathrm{H} & -5.67081800 & 1.59625000 & -2.09251900 \\ \mathrm{C} & -0.65685200 & -2.04654900 & 1.85002500 \\ \mathrm{C} & -1.17599600 & -2.65626400 & 3.11231500 \\ \mathrm{H} & -0.52281300 & -2.42014600 & 3.95941700 \\ \mathrm{H} & -2.17944800 & -2.28408600 & 3.32064600 \\ \mathrm{H} & -1.20663400 & -3.74777300 & 3.02982700 \\ \mathrm{~N} & 0.51873400 & -2.46595300 & 1.39540700 \\ \mathrm{O} & 1.22632400 & -3.27082700 & 2.07113400 \\ \mathrm{O} & 0.94880100 & -2.12030300 & 0.22849000 \\ \mathrm{C} & -3.34060600 & 1.67172600 & 1.97498100 \\ \mathrm{C} & -2.91113000 & 0.89837000 & 2.61995300 \\ \mathrm{H} & -4.47070000 & 2.35665200 & 2.74914600 \\ \mathrm{C} & -5.27877300 & 1.65587700 & 2.97843800 \\ \mathrm{H} & -4.08942100 & 2.76431900 & 3.69087300 \\ \mathrm{H} & -4.89662900 & 3.19044300 & 2.18046900 \\ \mathrm{H} & -2.22751600 & 2.66763100 & 1.62273100 \\ \mathrm{C} & -1.41141000 & 2.15894500 & 1.10222700 \\ \mathrm{H} & -2.61856500 & 3.45683200 & 0.96766800 \\ \mathrm{H} & -1.83122200 & 3.14356300 & 2.52650800 \\ \mathrm{H} & -3.28724200 & -2.34440600 & -1.09136700 \\ \mathrm{C} & -2.21081300 & -2.33986000 & -0.86519500 \\ \mathrm{H} & -3.96367800 & -3.38550000 & -0.18708200 \\ \mathrm{H} & -3.81816900 & -3.14882100 & 0.86890000 \\ \mathrm{H} & -3.93979000 & -3.41636600 & -0.39156700 \\ \mathrm{H} & -2.35106000 & -2.75527300 & -2.55559800 \\ \mathrm{H} & -2.02335300 & -3.23604700 \\ \mathrm{H} & -3.874900 & 1.36839500 & -1.78689000 \\ \mathrm{H} & 1.21889800 & -0.80152500 \\ \mathrm{H} & 2.45180000 & -0.13771300\end{array}$




$\begin{array}{lrrr}\mathrm{C} & 1.14589300 & 0.92929300 & -1.48060200 \\ \mathrm{C} & 3.70778800 & 2.77392500 & 0.62269500 \\ \mathrm{C} & 1.48692400 & 3.43067900 & -0.18399900 \\ \mathrm{C} & 0.09844000 & 1.91263000 & -1.48931200 \\ \mathrm{C} & 0.91148200 & -0.29957800 & -2.17593300 \\ \mathrm{C} & 3.83591100 & 3.97987300 & 1.24834500 \\ \mathrm{H} & 4.49995600 & 2.03935000 & 0.71535800 \\ \mathrm{C} & 1.66334000 & 4.68408100 & 0.48076100 \\ \mathrm{C} & 0.30188700 & 3.13618900 & -0.85798800 \\ \mathrm{C} & -1.14278500 & 1.61696100 & -2.13342900 \\ \mathrm{H} & 1.68418200 & -1.06059600 & -2.19946300 \\ \mathrm{C} & -0.28148100 & -0.53844500 & -2.79079700 \\ \mathrm{C} & 2.80420500 & 4.95908300 & 1.17214700 \\ \mathrm{H} & 4.73220700 & 4.19418700 & 1.82230000 \\ \mathrm{H} & 0.85526400 & 5.40875700 & 0.42554900 \\ \mathrm{H} & -0.49547400 & 3.87664400 & -0.87275200 \\ \mathrm{C} & -1.32942500 & 0.42720500 & -2.76642400 \\ \mathrm{H} & -1.93701900 & 2.35810600 & -2.09137600 \\ \mathrm{H} & -0.44094400 & -1.48501400 & -3.29923700 \\ \mathrm{H} & 2.92801000 & 5.91182000 & 1.67676200 \\ \mathrm{H} & -2.27812600 & 0.20971800 & -3.24640200 \\ \mathrm{H} & 2.17577600 & -1.22430200 & 0.16112600\end{array}$

\section{Structure 14}

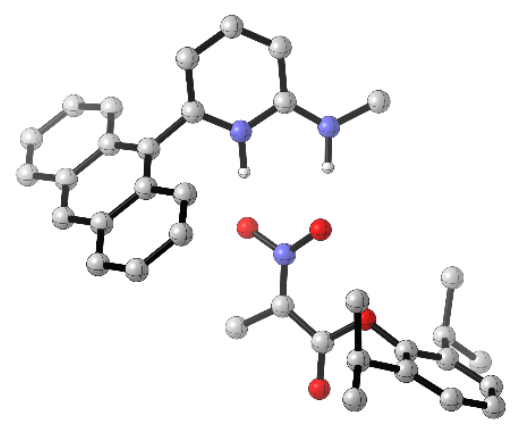

- Thermochemistry -

Zero-point correction=

Thermal correction to Energy=

Thermal correction to Enthalpy=

Thermal correction to Gibbs Free Energy=

Sum of electronic and zero-point Energies=

Sum of electronic and thermal Energies=

Sum of electronic and thermal Enthalpies=

Sum of electronic and thermal Free Energies=
0.659872 (Hartree/Particle)

0.698620

0.699564

0.586816

$-1819.686433$

$-1819.647685$

$-1819.646741$

$-1819.759489$ 
Number of Imaginary Frequencies $=0$

E (Single Point Energy) [SMD ${ }_{\text {(Toluene) }}$ M06-2X/6-311++G(2d,2p)] $=-1820.947633$

$\begin{array}{lrrr}\mathrm{C} & -3.36114200 & 1.21441700 & 0.82316100 \\ \mathrm{C} & -4.15663200 & 1.86468400 & 1.74793800 \\ \mathrm{C} & -3.83699900 & 3.18454500 & 2.09853400 \\ \mathrm{C} & -2.73796800 & 3.81892700 & 1.56393700 \\ \mathrm{H} & -4.45599800 & 3.71112500 & 2.81870700 \\ \mathrm{C} & -1.92310100 & 3.10925700 & 0.65140200 \\ \mathrm{H} & -2.47325500 & 4.82850900 & 1.85015000 \\ \mathrm{~N} & -2.29212400 & 1.85459200 & 0.29486500 \\ \mathrm{H} & -1.66844800 & 1.33520600 & -0.48920700 \\ \mathrm{~N} & -0.80284300 & 3.59063300 & 0.09428000 \\ \mathrm{H} & -0.15664600 & 2.86584300 & -0.25381700 \\ \mathrm{C} & -0.25907200 & 4.88022600 & 0.46166800 \\ \mathrm{H} & 0.66778400 & 5.02520700 & -0.09258200 \\ \mathrm{H} & -0.95020400 & 5.68475900 & 0.19068400 \\ \mathrm{H} & -0.03823300 & 4.94840500 & 1.53469200 \\ \mathrm{C} & 2.26424900 & -0.97066700 & -1.04342100 \\ \mathrm{O} & 2.80369700 & -1.99883600 & -1.38939800 \\ \mathrm{O} & 2.87778500 & -0.06482000 & -0.23977200 \\ \mathrm{C} & 4.22798000 & -0.25548200 & 0.03864500 \\ \mathrm{C} & 5.15557600 & 0.32432100 & -0.82969700 \\ \mathrm{C} & 4.57538900 & -0.93611700 & 1.20563600 \\ \mathrm{C} & 6.50293400 & 0.21845400 & -0.48064100 \\ \mathrm{C} & 5.93427600 & -1.01674800 & 1.51482300 \\ \mathrm{C} & 6.88835200 & -0.44240900 & 0.68149000 \\ \mathrm{H} & 7.26186300 & 0.64955000 & -1.12534500 \\ \mathrm{H} & 6.25289800 & -1.54008100 & 2.41056600 \\ \mathrm{H} & 7.94130400 & -0.51598000 & 0.93602100 \\ \mathrm{C} & 0.91398500 & -0.59824700 & -1.47605300 \\ \mathrm{C} & 0.18609100 & -1.49739900 & -2.42406500 \\ \mathrm{H} & -0.03427000 & -0.98648300 & -3.36645200 \\ \mathrm{H} & 0.80065900 & -2.37547300 & -2.62037100 \\ \mathrm{H} & -0.77252400 & -1.80528600 & -1.99236700 \\ \mathrm{~N} & 0.27100000 & 0.47113000 & -1.03048500 \\ \mathrm{O} & 0.68928700 & 1.26868700 & -0.13351200 \\ \mathrm{O} & -0.91240100 & 0.69725700 & -1.51518000 \\ \mathrm{C} & 3.48172300 & -1.52201800 & 2.08026000 \\ \mathrm{H} & 2.79097900 & -2.04773400 & 1.40581300 \\ \mathrm{H} & 2.70865100 & -0.40214500 & 2.79492900 \\ & 1.93659600 & 0.27574300 & 2.07728900 \\ \mathrm{H} & & -0.82518600 & 3.44287200\end{array}$




$\begin{array}{lrrr}\mathrm{H} & 3.39201800 & 0.17680800 & 3.42617600 \\ \mathrm{C} & 3.99981100 & -2.54114700 & 3.09389500 \\ \mathrm{H} & 4.58883800 & -3.32735000 & 2.61204200 \\ \mathrm{H} & 4.62471300 & -2.06078600 & 3.85523400 \\ \mathrm{H} & 3.15806300 & -3.01142600 & 3.61144700 \\ \mathrm{C} & 4.66669500 & 1.04479800 & -2.07526400 \\ \mathrm{H} & 3.93012800 & 0.38913900 & -2.55895500 \\ \mathrm{C} & 3.95853100 & 2.35805400 & -1.70586700 \\ \mathrm{H} & 3.61289600 & 2.86660700 & -2.61268600 \\ \mathrm{H} & 3.09654200 & 2.17710000 & -1.05808400 \\ \mathrm{H} & 4.65676100 & 3.02536200 & -1.18778000 \\ \mathrm{C} & 5.77851400 & 1.30997400 & -3.08942500 \\ \mathrm{H} & 6.31802700 & 0.39397800 & -3.34887500 \\ \mathrm{H} & 5.35193800 & 1.72721300 & -4.00637500 \\ \mathrm{H} & 6.50157100 & 2.03753800 & -2.70342700 \\ \mathrm{H} & -5.00281800 & 1.34740200 & 2.18245700 \\ \mathrm{C} & -3.60674700 & -0.18037500 & 0.37355400 \\ \mathrm{C} & -2.59391100 & -1.15111800 & 0.53791900 \\ \mathrm{C} & -4.84832000 & -0.51425800 & -0.20898600 \\ \mathrm{C} & -1.32207400 & -0.87066400 & 1.13496600 \\ \mathrm{C} & -2.83881300 & -2.49569200 & 0.09617800 \\ \mathrm{C} & -5.08661100 & -1.87374500 & -0.60753700 \\ \mathrm{C} & -5.88502200 & 0.44204400 & -0.46989900 \\ \mathrm{C} & -0.35318000 & -1.82778500 & 1.20671000 \\ \mathrm{H} & -1.09458300 & 0.12517000 & 1.49755100 \\ \mathrm{C} & -1.80160200 & -3.47554900 & 0.20925400 \\ \mathrm{C} & -4.07922800 & -2.82367900 & -0.44820300 \\ \mathrm{C} & -6.34682800 & -2.22872000 & -1.18386600 \\ \mathrm{H} & -5.71492300 & 1.48637800 & -0.23453000 \\ \mathrm{C} & -7.06996100 & 0.06481300 & -1.03047300 \\ \mathrm{C} & -0.58599100 & -3.15057300 & 0.73026200 \\ \mathrm{H} & 0.61468400 & -1.56931000 & 1.62561700 \\ \mathrm{H} & -2.00642000 & -4.48091500 & -0.14867900 \\ \mathrm{H} & -4.26191600 & -3.84701100 & -0.76961200 \\ \mathrm{C} & -7.31613600 & -1.29378800 & -1.38336000 \\ \mathrm{H} & -6.50524000 & -3.26612500 & -1.46596600 \\ \mathrm{H} & -7.83438300 & 0.81130100 & -1.22263800 \\ \mathrm{H} & 0.20845300 & -3.88798300 & 0.78630300 \\ \mathrm{H} & -8.26818300 & -1.57115100 & -1.82399300\end{array}$




\section{Structure 15}

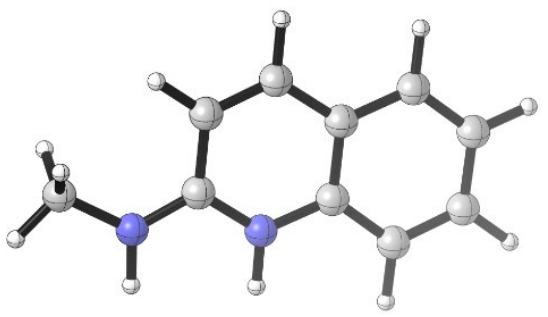

- Thermochemistry -

Zero-point correction $=$

0.196762 (Hartree/Particle)

Thermal correction to Energy=

0.206608

Thermal correction to Enthalpy=

0.207552

Thermal correction to Gibbs Free Energy=

0.161812

Sum of electronic and zero-point Energies $=$

$-496.577522$

Sum of electronic and thermal Energies=

$-496.567677$

Sum of electronic and thermal Enthalpies=

$-496.566732$

Sum of electronic and thermal Free Energies=

$-496.612472$

Number of Imaginary Frequencies $=0$

E (Single Point Energy) [SMD(Toluene)M06-2X/6-311++G(2d,2p)] = -496.983637

$\begin{array}{lrrr}\mathrm{C} & -1.82000100 & -1.49481100 & -0.00001700 \\ \mathrm{C} & -0.75949000 & -0.58175700 & -0.00000700 \\ \mathrm{C} & -2.32340500 & 1.27190400 & -0.00001800 \\ \mathrm{C} & -3.36949900 & 0.37632500 & -0.00002800 \\ \mathrm{C} & -3.11261400 & -1.00777000 & -0.00002700 \\ \mathrm{H} & -1.62724400 & -2.56367300 & -0.00001700 \\ \mathrm{H} & -2.50548900 & 2.34222800 & -0.00001800 \\ \mathrm{H} & -4.39318500 & 0.73274900 & -0.00003600 \\ \mathrm{H} & -3.94215700 & -1.70675300 & -0.00003500 \\ \mathrm{C} & -0.99189300 & 0.80670400 & -0.00000700 \\ \mathrm{C} & 0.14271800 & 1.67245200 & 0.00000400 \\ \mathrm{C} & 1.41677000 & 1.19199700 & 0.00001500 \\ \mathrm{H} & -0.02284300 & 2.74623800 & 0.00000400\end{array}$




$\begin{array}{lrrr}\mathrm{C} & 1.63566200 & -0.21922100 & 0.00001500 \\ \mathrm{H} & 2.27090800 & 1.85558800 & 0.00002400 \\ \mathrm{~N} & 0.55677300 & -1.02670900 & 0.00000400 \\ \mathrm{H} & 0.69758500 & -2.03268100 & 0.00000500 \\ \mathrm{~N} & 2.85612000 & -0.75700700 & 0.00002700 \\ \mathrm{H} & 2.94606400 & -1.76464300 & 0.00002500 \\ \mathrm{C} & 4.09017400 & 0.02569900 & 0.00002700 \\ \mathrm{H} & 4.92723800 & -0.67004100 & 0.00001700 \\ \mathrm{H} & 4.15416700 & 0.64894100 & -0.89545400 \\ \mathrm{H} & 4.15417600 & 0.64892700 & 0.89551700\end{array}$

\section{Structure 16}

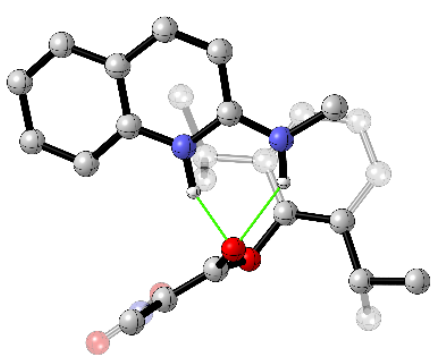

- Thermochemistry -

Zero-point correction $=$

0.533488 (Hartree/Particle)

Thermal correction to Energy=

0.564551

Thermal correction to Enthalpy=

0.565495

Thermal correction to Gibbs Free Energy=

0.472254

Sum of electronic and zero-point Energies $=$

$-1435.269607$

Sum of electronic and thermal Energies $=$

$-1435.238544$

Sum of electronic and thermal Enthalpies=

$-1435.237600$

Sum of electronic and thermal Free Energies=

$-1435.330841$

Number of Imaginary Frequencies $=0$

E (Single Point Energy) $\left[\mathrm{SMD}_{\text {(Toluene) }}\right.$ M06-2X/6-311++G(2d,2p)] $=-1436.291344$ 


$\begin{array}{lrrr}\mathrm{C} & -2.99828400 & 0.94647800 & -0.57129200 \\ \mathrm{C} & -2.66005100 & -0.41469700 & -0.65638600 \\ \mathrm{C} & -4.89451500 & -1.03393700 & 0.06146100 \\ \mathrm{C} & -5.22911300 & 0.29914600 & 0.14014200 \\ \mathrm{C} & -4.27348800 & 1.28758800 & -0.17342900 \\ \mathrm{H} & -2.24358300 & 1.69865500 & -0.79088000 \\ \mathrm{H} & -5.61662100 & -1.80586200 & 0.31206500 \\ \mathrm{H} & -6.22515900 & 0.59404300 & 0.45149500 \\ \mathrm{H} & -4.54004100 & 2.33638500 & -0.09404300 \\ \mathrm{C} & -3.59659300 & -1.41757800 & -0.33547600 \\ \mathrm{C} & -3.15485600 & -2.77543000 & -0.39520700 \\ \mathrm{C} & -1.87551700 & -3.09996600 & -0.72472000 \\ \mathrm{H} & -3.86352000 & -3.56239700 & -0.15057600 \\ \mathrm{C} & -0.94911900 & -2.05271500 & -1.04876100 \\ \mathrm{H} & -1.53882200 & -4.12818100 & -0.74747800 \\ \mathrm{~N} & -1.39076800 & -0.78473200 & -1.02918100 \\ \mathrm{H} & -0.67790300 & -0.06713100 & -1.33448100 \\ \mathrm{~N} & 0.31973600 & -2.26590200 & -1.38576900 \\ \mathrm{H} & 0.90711800 & -1.42974600 & -1.47667900 \\ \mathrm{C} & 0.92117300 & -3.58315900 & -1.44395500 \\ \mathrm{H} & 1.97324100 & -3.45652200 & -1.69623100 \\ \mathrm{H} & 0.86595400 & -4.08694700 & -0.47351800 \\ \mathrm{H} & 0.44316900 & -4.19912200 & -2.21261800 \\ \mathrm{C} & 0.86224300 & 1.48517300 & -0.82321800 \\ \mathrm{H} & 0.78271700 & 0.53193100 & -1.66077100 \\ \mathrm{C} & 1.61144400 & 1.27798300 & 0.30549500 \\ \mathrm{O} & -0.63114500 & 0.90947200 & 1.22861900 \\ \mathrm{C} & 0.16950100 & 1.15033300 & 3.18478100 \\ \mathrm{C} & & & \end{array}$




$\begin{array}{lrrr}\mathrm{H} & 0.98516200 & 1.78947500 & 2.84292400 \\ \mathrm{H} & -0.64338900 & 1.79912200 & 3.52562000 \\ \mathrm{H} & 0.50956700 & 0.53893200 & 4.02889100 \\ \mathrm{C} & -1.51906500 & -0.58958500 & 2.48811000 \\ \mathrm{H} & -1.76816600 & -1.37223100 & 1.76221600 \\ \mathrm{H} & -1.32744100 & -1.07837400 & 3.45030600 \\ \mathrm{H} & -2.39678200 & 0.05248800 & 2.61387600 \\ \mathrm{C} & 3.90684800 & -0.03079600 & -0.68019400 \\ \mathrm{H} & 3.31741000 & 0.52846500 & -1.41562800 \\ \mathrm{C} & 4.73954500 & 0.97329300 & 0.13008800 \\ \mathrm{H} & 5.42942900 & 1.51422300 & -0.52583700 \\ \mathrm{H} & 4.09626900 & 1.70074300 & 0.63092800 \\ \mathrm{H} & 5.32975400 & 0.44862300 & 0.88976800 \\ \mathrm{C} & 4.81942700 & -0.99154700 & -1.44116500 \\ \mathrm{H} & 4.24773600 & -1.74656800 & -1.99267700 \\ \mathrm{H} & 5.42535800 & -0.43507200 & -2.16191700 \\ \mathrm{H} & 5.50933700 & -1.51057100 & -0.76633600\end{array}$

Structure 17

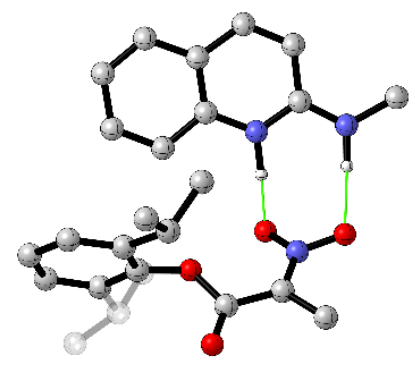

- Thermochemistry -

Zero-point correction=

Thermal correction to Energy=

Thermal correction to Enthalpy=

Thermal correction to Gibbs Free Energy=

Sum of electronic and zero-point Energies $=$

Sum of electronic and thermal Energies=

Sum of electronic and thermal Enthalpies=

Sum of electronic and thermal Free Energies=
0.531833 (Hartree/Particle)

0.563092

0.564036

0.468886

$-1435.282567$

$-1435.251308$

$-1435.250364$

$-1435.345514$ 
Number of Imaginary Frequencies $=0$

E (Single Point Energy) $\left[\operatorname{SMD}_{(\text {Toluene) }}\right.$ M06-2X/6-311++G(2d,2p)] $=-1436.301503$

$\begin{array}{lrrr}\mathrm{C} & -0.45160700 & -1.69624000 & -0.70690400 \\ \mathrm{C} & -1.83678500 & -1.57231700 & -0.49376900 \\ \mathrm{C} & -1.98346000 & -3.93816100 & 0.02150300 \\ \mathrm{C} & -0.62462600 & -4.05428000 & -0.16756900 \\ \mathrm{C} & 0.14273100 & -2.92514900 & -0.53107300 \\ \mathrm{H} & 0.11969600 & -0.81126300 & -0.96955500 \\ \mathrm{H} & -2.58258700 & -4.80090000 & 0.29957000 \\ \mathrm{H} & -0.13818100 & -5.01470600 & -0.03508000 \\ \mathrm{H} & 1.21823300 & -3.01332800 & -0.66187700 \\ \mathrm{C} & -2.61936700 & -2.68639000 & -0.13388200 \\ \mathrm{C} & -4.01668300 & -2.46354100 & 0.06273100 \\ \mathrm{C} & -4.56895600 & -1.22553400 & -0.06281500 \\ \mathrm{H} & -4.64667200 & -3.30903900 & 0.32729600 \\ \mathrm{C} & -3.72546300 & -0.11089000 & -0.39406000 \\ \mathrm{H} & -5.62678500 & -1.06031400 & 0.09716400 \\ \mathrm{~N} & -2.42483600 & -0.33892700 & -0.61142100 \\ \mathrm{H} & -1.78916300 & 0.50884800 & -0.90736300 \\ \mathrm{~N} & -4.16045800 & 1.14505000 & -0.49572800 \\ \mathrm{H} & -3.44452500 & 1.88890000 & -0.58903900 \\ \mathrm{C} & -5.53170100 & 1.53704000 & -0.24479600 \\ \mathrm{H} & -5.59630100 & 2.61700800 & -0.37260000 \\ \mathrm{H} & -6.21740000 & 1.06358100 & -0.95513800 \\ \mathrm{H} & -5.84660500 & 1.28944300 & 0.77545800 \\ \mathrm{C} & 1.34241300 & 2.31457000 & 0.30879100 \\ \mathrm{O} & 2.33130900 & 2.77756900 & 0.83092100 \\ \mathrm{O} & 1.30196900 & 1.03212100 & -0.15170900 \\ \mathrm{C} & 2.28843800 & 0.12105800 & 0.19989100 \\ \mathrm{C} & 2.26999000 & -0.43926100 & 1.48381600 \\ \mathrm{C} & 3.15339600 & -0.29340300 & -0.81638500 \\ \mathrm{C} & 3.14398700 & -1.50288900 & 1.71793200 \\ \mathrm{C} & 4.00476500 & -1.36446700 & -0.53270000 \\ \mathrm{C} & 3.99114300 & -1.97182400 & 0.71836700 \\ \mathrm{H} & 3.16928300 & -1.97086900 & 2.69624400 \\ \mathrm{H} & 4.69533900 & -1.71847600 & -1.29120600 \\ \mathrm{H} & 4.65976800 & -2.80240400 & 0.92363200 \\ \mathrm{C} & 0.06425100 & 3.02387600 & 0.22111600 \\ \mathrm{C} & -0.12763700 & 4.26922100 & 1.02580300 \\ \mathrm{H} & -0.80530500 & 4.09532700 & 1.86936000\end{array}$




$\begin{array}{lrrr}\mathrm{H} & 0.83956900 & 4.60037200 & 1.40339600 \\ \mathrm{H} & -0.57642900 & 5.05677000 & 0.41361000 \\ \mathrm{~N} & -0.99007500 & 2.55799500 & -0.44347100 \\ \mathrm{O} & -0.89959200 & 1.59122700 & -1.29851900 \\ \mathrm{O} & -2.12851900 & 3.09111700 & -0.27355800 \\ \mathrm{C} & 3.14114700 & 0.42526000 & -2.15659900 \\ \mathrm{H} & 3.09784700 & 1.49939200 & -1.93451500 \\ \mathrm{C} & 1.89334500 & 0.07000300 & -2.97960600 \\ \mathrm{H} & 0.98380100 & 0.45113500 & -2.50809700 \\ \mathrm{H} & 1.96401800 & 0.51338000 & -3.97871300 \\ \mathrm{H} & 1.81225900 & -1.01745200 & -3.09916300 \\ \mathrm{C} & 4.40596300 & 0.16813200 & -2.97615600 \\ \mathrm{H} & 5.31341300 & 0.36964900 & -2.39874000 \\ \mathrm{H} & 4.44659500 & -0.86829900 & -3.33065500 \\ \mathrm{H} & 4.41109400 & 0.81437900 & -3.85863300 \\ \mathrm{C} & 1.33780200 & 0.10923200 & 2.55590500 \\ \mathrm{H} & 1.46310100 & 1.19878800 & 2.55546000 \\ \mathrm{C} & -0.13615700 & -0.19919900 & 2.25359400 \\ \mathrm{H} & -0.77476600 & 0.22053800 & 3.03830200 \\ \mathrm{H} & -0.44700800 & 0.22635200 & 1.29573900 \\ \mathrm{H} & -0.30077500 & -1.28279500 & 2.21804300 \\ \mathrm{C} & 1.68950000 & -0.38140600 & 3.96078100 \\ \mathrm{H} & 2.73807700 & -0.18916600 & 4.20658100 \\ \mathrm{H} & 1.06702800 & 0.13522500 & 4.69717300 \\ \mathrm{H} & 1.50106200 & -1.45583900 & 4.06711800\end{array}$

Structure 18
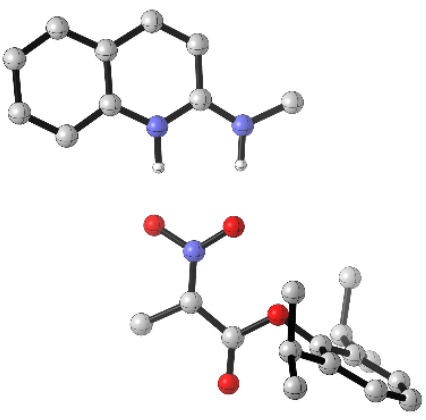

- Thermochemistry -

Zero-point correction=

0.531942 (Hartree/Particle)

Thermal correction to Energy=

0.563542

Thermal correction to Enthalpy=

0.564486

Thermal correction to Gibbs Free Energy=

0.466523 
Sum of electronic and zero-point Energies $=$

Sum of electronic and thermal Energies $=$

Sum of electronic and thermal Enthalpies $=$

Sum of electronic and thermal Free Energies=
$-1435.280927$

$-1435.249327$

$-1435.248382$

$-1435.346346$

Number of Imaginary Frequencies $=0$

E (Single Point Energy) $\left[\mathrm{SMD}_{\text {(Toluene) }} \mathrm{M} 06-2 \mathrm{X} / 6-311++\mathrm{G}(2 \mathrm{~d}, 2 \mathrm{p})\right]=-1436.301520$

$\begin{array}{lrrr}\mathrm{C} & 4.79593200 & -1.61435800 & 0.50675800 \\ \mathrm{C} & 4.67216600 & -0.32989800 & -0.05636300 \\ \mathrm{C} & 7.04239900 & -0.32437900 & -0.59004200 \\ \mathrm{C} & 7.16297000 & -1.57996600 & -0.03992000 \\ \mathrm{C} & 6.03271800 & -2.22098400 & 0.50941500 \\ \mathrm{H} & 3.91410400 & -2.09136000 & 0.92468200 \\ \mathrm{H} & 7.90353100 & 0.18241700 & -1.01672700 \\ \mathrm{H} & 8.12527500 & -2.07999100 & -0.02658700 \\ \mathrm{H} & 6.13832500 & -3.21098400 & 0.94131100 \\ \mathrm{C} & 5.79085800 & 0.32649300 & -0.60770500 \\ \mathrm{C} & 5.58171500 & 1.63080200 & -1.15238100 \\ \mathrm{C} & 4.35477600 & 2.21475200 & -1.14716900 \\ \mathrm{H} & 6.42998000 & 2.15837500 & -1.58086700 \\ \mathrm{C} & 3.23742500 & 1.50744700 & -0.58335900 \\ \mathrm{H} & 4.19608500 & 3.20049900 & -1.56520900 \\ \mathrm{~N} & 3.44667800 & 0.28756200 & -0.06580000 \\ \mathrm{H} & 2.63456200 & -0.23101900 & 0.43509000 \\ \mathrm{~N} & 2.00455600 & 2.00368400 & -0.53891900 \\ \mathrm{H} & 1.26275500 & 1.32787500 & -0.27156400 \\ \mathrm{C} & 1.63394000 & 3.28779100 & -1.09754800 \\ \mathrm{H} & 0.56599900 & 3.42658200 & -0.92953600 \\ \mathrm{H} & 2.17018700 & 4.10402900 & -0.60286400 \\ \mathrm{H} & 1.82511000 & 3.33481700 & -2.17580700 \\ \mathrm{C} & -1.93593300 & -1.14776600 & 1.25217700 \\ \mathrm{O} & -2.83974400 & -1.62171300 & 1.90500000 \\ \mathrm{O} & -2.15928700 & -0.36894100 & 0.16337400 \\ \mathrm{C} & -3.47205900 & -0.01819200 & -0.13202200 \\ \mathrm{C} & -3.93517400 & 1.20372700 & 0.36025300 \\ \mathrm{C} & -4.21410000 & -0.85204900 & -0.96953700 \\ \mathrm{C} & -5.21370700 & 1.60396600 & -0.03146300 \\ \mathrm{C} & -5.48733900 & -0.41094700 & -1.33461900 \\ \mathrm{C} & -5.98101000 & 0.80517600 & -0.87323600\end{array}$




$\begin{array}{lrrr}\mathrm{H} & -5.61968500 & 2.54358500 & 0.32910800 \\ \mathrm{H} & -6.10507000 & -1.02648200 & -1.98049400 \\ \mathrm{H} & -6.97450000 & 1.13013500 & -1.16705900 \\ \mathrm{C} & -0.52376400 & -1.35198100 & 1.58722000 \\ \mathrm{C} & -0.18394000 & -2.16748400 & 2.79442900 \\ \mathrm{H} & 0.33279700 & -1.56148000 & 3.54565300 \\ \mathrm{H} & -1.10323500 & -2.56772400 & 3.22026700 \\ \mathrm{H} & 0.48895100 & -2.98956300 & 2.53147400 \\ \mathrm{~N} & 0.48236900 & -0.81021000 & 0.91043200 \\ \mathrm{O} & 0.36101600 & -0.13186600 & -0.16108300 \\ \mathrm{O} & 1.67782000 & -0.99176600 & 1.36431900 \\ \mathrm{C} & -3.60618000 & -2.15606500 & -1.45550900 \\ \mathrm{H} & -3.16202000 & -2.64551900 & -0.57941000 \\ \mathrm{C} & -2.48647400 & -1.88490200 & -2.47242900 \\ \mathrm{H} & -1.69474900 & -1.26842700 & -2.03898100 \\ \mathrm{H} & -2.04529000 & -2.82893400 & -2.81028800 \\ \mathrm{H} & -2.89435400 & -1.36852300 & -3.34888400 \\ \mathrm{C} & -4.63809800 & -3.11610900 & -2.04566300 \\ \mathrm{H} & -5.46390900 & -3.30002300 & -1.35160600 \\ \mathrm{H} & -5.05489200 & -2.72540800 & -2.98104100 \\ \mathrm{H} & -4.16369000 & -4.07499100 & -2.27466000 \\ \mathrm{C} & -3.03780000 & 2.03096900 & 1.26600700 \\ \mathrm{H} & -2.62585500 & 1.34861500 & 2.02153900 \\ \mathrm{C} & -1.85666100 & 2.61882700 & 0.47593500 \\ \mathrm{H} & -1.21362600 & 3.20631100 & 1.14256100 \\ \mathrm{H} & -1.26037900 & 1.82200900 & 0.02126500 \\ \mathrm{H} & -2.22891600 & 3.28313500 & -0.31319600 \\ \mathrm{C} & -3.78644200 & 3.13930300 & 2.00443100 \\ \mathrm{H} & -4.64809200 & 2.74818100 & 2.55372900 \\ \mathrm{H} & -3.11772500 & 3.62752300 & 2.71971900 \\ \mathrm{H} & -4.14264300 & 3.90833000 & 1.30955100\end{array}$

Structure 19

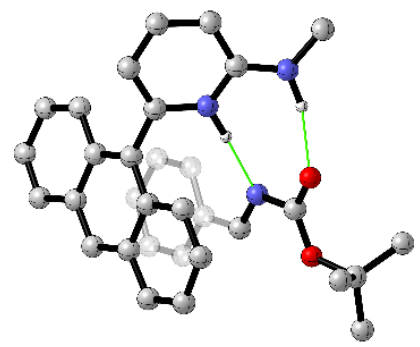

- Thermochemistry - 
Zero-point correction $=$

Thermal correction to Energy=

Thermal correction to Enthalpy=

Thermal correction to Gibbs Free Energy=

Sum of electronic and zero-point Energies=

Sum of electronic and thermal Energies=

Sum of electronic and thermal Enthalpies=

Sum of electronic and thermal Free Energies=
0.580651 (Hartree/Particle)

0.613467

0.614411

0.517458

$-1552.017476$

$-1551.984659$

$-1551.983715$

$-1552.080668$

Number of Imaginary Frequencies $=0$

E (Single Point Energy) $\left[\operatorname{SMD}_{\text {(Toluene) }}\right.$ M06-2X/6-311++G(2d,2p) $]=-1553.122742$

$\begin{array}{lrrr}\mathrm{C} & 0.66901700 & -1.49440800 & -1.10295700 \\ \mathrm{C} & -0.70003500 & -1.69341600 & -1.55406100 \\ \mathrm{C} & -1.24013700 & -2.98495400 & -1.48516200 \\ \mathrm{C} & -1.47443400 & -0.64169300 & -2.06412300 \\ \mathrm{C} & -2.54316800 & -3.22125400 & -1.90339300 \\ \mathrm{C} & -2.77153600 & -0.88172400 & -2.49262500 \\ \mathrm{C} & -3.30682600 & -2.16857700 & -2.40617600 \\ \mathrm{C} & 2.55679100 & -0.23699600 & -0.58662300 \\ \mathrm{O} & 3.04337700 & 0.86306500 & -0.38812900 \\ \mathrm{O} & 3.19146500 & -1.38790900 & -0.49126500 \\ \mathrm{C} & 4.63957100 & -1.46366600 & -0.22081100 \\ \mathrm{C} & 4.92490900 & -0.94194200 & 1.18070500 \\ \mathrm{H} & 4.65559800 & 0.11173500 & 1.27527600 \\ \mathrm{H} & 5.99334500 & -1.04875500 & 1.38942500 \\ \mathrm{H} & 4.37468600 & -1.53030600 & 1.92106500 \\ \mathrm{C} & 5.40361400 & -0.70245300 & -1.29756700 \\ \mathrm{H} & 5.09043900 & -1.03612800 & -2.29143300 \\ \mathrm{H} & 6.47075700 & -0.91612100 & -1.18953100 \\ \mathrm{H} & 5.25477300 & 0.37450400 & -1.21304900 \\ \mathrm{C} & 4.91245700 & -2.95855700 & -0.31261400 \\ \mathrm{H} & 5.96586300 & -3.15504600 & -0.09701100 \\ \mathrm{H} & 4.68673600 & -3.33132000 & -1.31543700 \\ \mathrm{H} & 4.30125600 & -3.50346300 & 0.41257000 \\ \mathrm{~N} & 1.20323200 & -0.32946200 & -0.97981800 \\ \mathrm{H} & 1.24460700 & -2.39427600 & -0.86832000\end{array}$




\begin{tabular}{|c|c|c|c|}
\hline 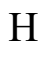 & -4.32416200 & -2.35048300 & -2.73835600 \\
\hline & -2.96105200 & -4.22086900 & -1.84736200 \\
\hline & -3.37158200 & -0.07004900 & -2.88985000 \\
\hline & -1.04301100 & 0.35280500 & -2.13445400 \\
\hline & -0.63222900 & -3.79789200 & -1.09508100 \\
\hline & 2.52551700 & 4.51312400 & -1.53895800 \\
\hline & 2.88850600 & 5.08409200 & -0.67708700 \\
\hline & 1.78775100 & 3.32898600 & -1.13169600 \\
\hline & 2.27816000 & 2.43176100 & -1.12309100 \\
\hline & 0.67086300 & 3.39881300 & -0.40258100 \\
\hline & 0.06208700 & 4.60249500 & 0.02434800 \\
\hline & -1.03500100 & 2.14248800 & 0.74757300 \\
\hline $\mathrm{C}$ & -1.05975600 & 4.53689900 & 0.81638400 \\
\hline $\mathrm{H}$ & 0.50470400 & 5.55080000 & -0.25025200 \\
\hline & -1.61526800 & 3.30279200 & 1.20291200 \\
\hline & -1.51361100 & 5.45821500 & 1.16763800 \\
\hline & -2.47826700 & 3.24629300 & 1.85432100 \\
\hline & 0.06918600 & 2.23137300 & -0.05354100 \\
\hline 0 & -1.50331500 & 0.77238000 & 1.05684100 \\
\hline & -2.81344400 & 0.37886200 & 0.70814700 \\
\hline $\mathrm{C}$ & -0.59872300 & -0.13427700 & 1.65180100 \\
\hline $\mathrm{C}$ & -3.75435500 & 1.23750200 & 0.05261300 \\
\hline $\mathrm{C}$ & -3.21823800 & -0.97548700 & 0.96802400 \\
\hline $\mathrm{C}$ & -0.99980400 & -1.49857700 & 1.83301500 \\
\hline $\mathrm{C}$ & 0.71373200 & 0.23811100 & 2.09248300 \\
\hline $\mathrm{C}$ & -5.00952300 & 0.79883700 & -0.25809700 \\
\hline $\mathrm{H}$ & -3.46508700 & 2.24944600 & -0.20763600 \\
\hline $\mathrm{C}$ & -4.54508100 & -1.39150500 & 0.63367300 \\
\hline $\mathrm{C}$ & -2.30108400 & -1.87857700 & 1.50293400 \\
\hline $\mathrm{C}$ & -0.05145700 & -2.44969100 & 2.32599400 \\
\hline $\mathrm{H}$ & 1.02354700 & 1.27703500 & 2.05175000 \\
\hline $\mathrm{C}$ & 1.58306400 & -0.69433600 & 2.57947300 \\
\hline $\mathrm{C}$ & -5.42249700 & -0.53006100 & 0.04762400 \\
\hline $\mathrm{H}$ & -5.70592500 & 1.47163100 & -0.74887500 \\
\hline $\mathrm{H}$ & -4.83136300 & -2.41598000 & 0.85555400 \\
\hline $\mathrm{H}$ & -2.60586400 & -2.91191300 & 1.65538200 \\
\hline $\mathrm{C}$ & 1.21144600 & -2.06729700 & 2.66979700 \\
\hline $\mathrm{H}$ & -0.36870000 & -3.48386300 & 2.43040700 \\
\hline $\mathrm{H}$ & 2.56567600 & -0.38160100 & 2.91748900 \\
\hline $\mathrm{H}$ & -6.42945800 & -0.84973100 & -0.19903300 \\
\hline $\mathrm{H}$ & 1.92412100 & -2.79408700 & 3.04636800 \\
\hline $\mathrm{H}$ & 0.46897800 & 1.34154200 & -0.41898600 \\
\hline $\mathrm{H}$ & 1.90120900 & 5.15766000 & -2.16358400 \\
\hline $\mathrm{H}$ & 3.38111500 & 4.18972100 & -2.12982200 \\
\hline
\end{tabular}


Structure 20

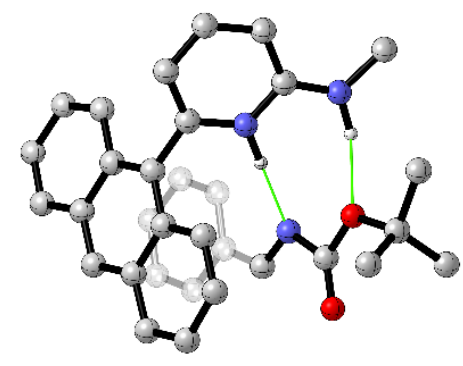

- Thermochemistry -

Zero-point correction $=$ 0.580680 (Hartree/Particle)

Thermal correction to Energy=

0.613253

Thermal correction to Enthalpy=

0.614197

Thermal correction to Gibbs Free Energy=

0.518435

Sum of electronic and zero-point Energies=

$-1552.015154$

Sum of electronic and thermal Energies=

$-1551.982581$

Sum of electronic and thermal Enthalpies=

$-1551.981637$

Sum of electronic and thermal Free Energies=

$-1552.077399$

Number of Imaginary Frequencies $=0$

E (Single Point Energy) $\left[\mathrm{SMD}_{(\text {Toluene) }} \mathrm{M} 06-2 \mathrm{X} / 6-311++\mathrm{G}(2 \mathrm{~d}, 2 \mathrm{p})\right]=-1553.122348$

$\begin{array}{lrrr}\mathrm{C} & -0.03211200 & -1.78782800 & 1.32953500 \\ \mathrm{C} & 1.30533100 & -1.40853400 & 1.75590900 \\ \mathrm{C} & 2.29017200 & -2.40493800 & 1.80499500 \\ \mathrm{C} & 1.62967400 & -0.09289700 & 2.11798100 \\ \mathrm{C} & 3.58623600 & -2.08898500 & 2.19189200 \\ \mathrm{C} & 2.92228300 & 0.21956200 & 2.51080300 \\ \mathrm{C} & 3.90070500 & -0.77678300 & 2.54165200 \\ \mathrm{C} & -2.20536700 & -1.52802100 & 0.69544200 \\ \mathrm{O} & -2.41185800 & -2.70033800 & 0.51863000\end{array}$




\begin{tabular}{|c|c|c|c|}
\hline $\mathrm{O}$ & -3.10066800 & -0.54017200 & 0.54621300 \\
\hline $\mathrm{C}$ & -4.48996100 & -0.85548100 & 0.16967500 \\
\hline $\mathrm{C}$ & -5.12436000 & -1.75848000 & 1.22173400 \\
\hline $\mathrm{H}$ & -4.69033700 & -2.75821300 & 1.20935300 \\
\hline $\mathrm{H}$ & -6.19610700 & -1.83941700 & 1.01854600 \\
\hline $\mathrm{H}$ & -5.00018100 & -1.32427100 & 2.21867400 \\
\hline $\mathrm{C}$ & -4.51599900 & -1.47225900 & -1.22319700 \\
\hline $\mathrm{H}$ & -4.02178700 & -0.80829700 & -1.94052500 \\
\hline $\mathrm{H}$ & -5.55583900 & -1.59788900 & -1.53829500 \\
\hline $\mathrm{H}$ & -4.03025200 & -2.44890100 & -1.23152500 \\
\hline $\mathrm{C}$ & -5.16099200 & 0.51218100 & 0.16216600 \\
\hline $\mathrm{H}$ & -6.19767400 & 0.41180500 & -0.16879900 \\
\hline $\mathrm{H}$ & -4.64346800 & 1.19463600 & -0.52051700 \\
\hline $\mathrm{H}$ & -5.16891700 & 0.94550400 & 1.16788900 \\
\hline $\mathrm{N}$ & -0.97408000 & -0.93997200 & 1.09857400 \\
\hline $\mathrm{H}$ & -0.23331000 & -2.85625100 & 1.19581300 \\
\hline $\mathrm{H}$ & 4.91216600 & -0.52684300 & 2.84632200 \\
\hline $\mathrm{H}$ & 4.34759200 & -2.86103500 & 2.22763600 \\
\hline $\mathrm{H}$ & 3.17457600 & 1.23652100 & 2.79143500 \\
\hline $\mathrm{H}$ & 0.85769300 & 0.67161800 & 2.10730000 \\
\hline $\mathrm{H}$ & 2.03122500 & -3.42443800 & 1.52965000 \\
\hline $\mathrm{C}$ & -3.55920500 & 3.22568300 & 1.65721900 \\
\hline $\mathrm{H}$ & -4.28268300 & 3.46710700 & 0.86985000 \\
\hline $\mathrm{N}$ & -2.54337700 & 2.29383000 & 1.19806100 \\
\hline $\mathrm{H}$ & -2.66738400 & 1.30338800 & 1.39561600 \\
\hline $\mathrm{C}$ & -1.64592800 & 2.61592200 & 0.25648800 \\
\hline $\mathrm{C}$ & -1.57603700 & 3.87310300 & -0.38396300 \\
\hline $\mathrm{C}$ & 0.26459400 & 1.87378200 & -1.00456700 \\
\hline $\mathrm{C}$ & -0.59744800 & 4.08442800 & -1.32861900 \\
\hline $\mathrm{H}$ & -2.30051700 & 4.63928000 & -0.14181100 \\
\hline $\mathrm{C}$ & 0.33656000 & 3.08488000 & -1.65315700 \\
\hline $\mathrm{H}$ & -0.55242900 & 5.04201900 & -1.83777300 \\
\hline $\mathrm{H}$ & 1.10865400 & 3.24478400 & -2.39527700 \\
\hline $\mathrm{N}$ & -0.72494900 & 1.67987100 & -0.08365200 \\
\hline $\mathrm{C}$ & 1.20966000 & 0.74816200 & -1.18841100 \\
\hline $\mathrm{C}$ & 2.56837700 & 0.92798300 & -0.84851600 \\
\hline $\mathrm{C}$ & 0.71784400 & -0.49919000 & -1.62466200 \\
\hline $\mathrm{C}$ & 3.10816900 & 2.15657100 & -0.34722100 \\
\hline $\mathrm{C}$ & 3.45762900 & -0.19550500 & -0.95231800 \\
\hline $\mathrm{C}$ & 1.61160400 & -1.61976100 & -1.66574000 \\
\hline $\mathrm{C}$ & -0.64069000 & -0.71097800 & -2.03257000 \\
\hline $\mathrm{C}$ & 4.43180900 & 2.26601400 & -0.02987100 \\
\hline $\mathrm{H}$ & 2.45715500 & 3.01318600 & -0.21233000 \\
\hline $\mathrm{C}$ & 4.83840600 & -0.03574200 & -0.61694800 \\
\hline $\mathrm{C}$ & 2.95576400 & -1.43632600 & -1.34213100 \\
\hline $\mathrm{C}$ & 1.10335500 & -2.91081000 & -2.01401100 \\
\hline
\end{tabular}




$\begin{array}{lrrc}\mathrm{H} & -1.32590700 & 0.13116300 & -2.07790900 \\ \mathrm{C} & -1.08108500 & -1.95579600 & -2.37823100 \\ \mathrm{C} & 5.31843600 & 1.16071100 & -0.17642600 \\ \mathrm{H} & 4.81733400 & 3.21026300 & 0.34194700 \\ \mathrm{H} & 5.49291700 & -0.89709200 & -0.71911800 \\ \mathrm{H} & 3.63057600 & -2.28889400 & -1.38366100 \\ \mathrm{C} & -0.20763400 & -3.08141400 & -2.34360300 \\ \mathrm{H} & 1.79275800 & -3.75080600 & -2.01627300 \\ \mathrm{H} & -2.10988400 & -2.09694000 & -2.69198300 \\ \mathrm{H} & 6.36808000 & 1.27736700 & 0.07180400 \\ \mathrm{H} & -0.58921500 & -4.06283600 & -2.60305900 \\ \mathrm{H} & -0.76561400 & 0.75183200 & 0.40052700 \\ \mathrm{H} & -3.09248500 & 4.14638700 & 2.01681100 \\ \mathrm{H} & -4.08843600 & 2.76771900 & 2.49208000\end{array}$

\section{Structure syn-product}

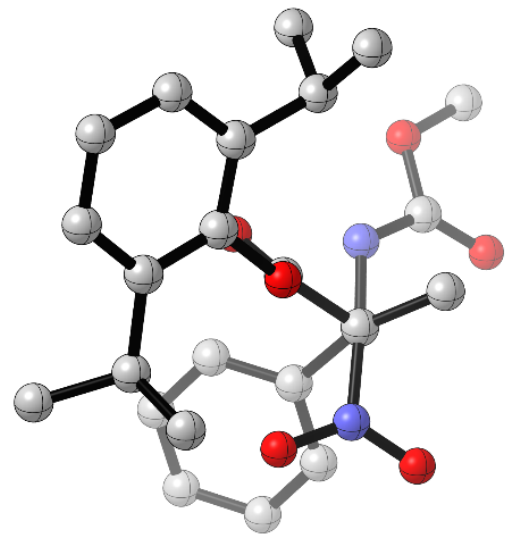

- Thermochemistry -

Zero-point correction $=$

Thermal correction to Energy=

Thermal correction to Enthalpy=

Thermal correction to Gibbs Free Energy=

Sum of electronic and zero-point Energies=

Sum of electronic and thermal Energies=

Sum of electronic and thermal Enthalpies=

Sum of electronic and thermal Free Energies=
0.521135 (Hartree/Particle)

0.552999

0.553943

0.456508

$-1492.279637$

$-1492.247774$

$-1492.246829$

$-1492.344264$ 
Number of Imaginary Frequencies $=0$

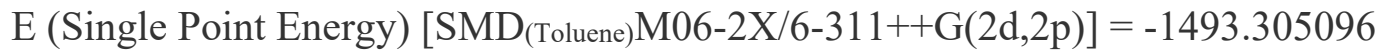

$\begin{array}{lrrr}\mathrm{C} & -0.25122600 & -0.02194600 & 0.12719300 \\ \mathrm{O} & -0.17832800 & -0.04266800 & -1.07398900 \\ \mathrm{O} & -1.39732600 & -0.06162300 & 0.82115500 \\ \mathrm{C} & -2.59837500 & -0.07714500 & 0.08352900 \\ \mathrm{C} & -3.05777800 & -1.29844100 & -0.41277000 \\ \mathrm{C} & -3.27295000 & 1.13553800 & -0.05402300 \\ \mathrm{C} & -4.27218900 & -1.27326600 & -1.09980600 \\ \mathrm{C} & -4.48516700 & 1.10549000 & -0.74767400 \\ \mathrm{C} & -4.97690900 & -0.08509800 & -1.26834800 \\ \mathrm{H} & -4.67208600 & -2.19372100 & -1.51165500 \\ \mathrm{H} & -5.05083500 & 2.02254200 & -0.87772500 \\ \mathrm{H} & -5.92012700 & -0.08988000 & -1.80571300 \\ \mathrm{C} & 0.94164500 & 0.17022600 & 1.06573000 \\ \mathrm{C} & 0.73548200 & 1.40828100 & 1.93550500 \\ \mathrm{H} & 1.63162100 & 1.60678900 & 2.52368100 \\ \mathrm{H} & 0.54204200 & 2.26992900 & 1.29030100 \\ \mathrm{H} & -0.12071000 & 1.26596400 & 2.59778300 \\ \mathrm{~N} & 1.03177400 & -1.04800700 & 1.95774500 \\ \mathrm{O} & 1.70654400 & -0.93627700 & 2.96173300 \\ \mathrm{O} & 0.49198800 & -2.06685100 & 1.57628600 \\ \mathrm{C} & -2.68621100 & 2.41944600 & 0.50739700 \\ \mathrm{H} & -2.11641200 & 2.15121200 & 1.40447000 \\ \mathrm{C} & -3.75525900 & 3.43182000 & 0.92401600 \\ \mathrm{H} & -4.49983700 & 2.98013300 & 1.58589700 \\ \mathrm{H} & -3.28853300 & 4.26943500 & 1.45088800 \\ \mathrm{H} & -4.27581000 & 3.84575700 & 0.05403200 \\ \mathrm{C} & -1.71775100 & 3.05946700 & -0.49943900 \\ \mathrm{H} & -0.92040700 & 2.37386000 & -0.80178600 \\ \mathrm{H} & -2.25880400 & 3.35012400 & -1.40662500 \\ \mathrm{H} & -1.25925600 & 3.95865700 & -0.07298100 \\ \mathrm{C} & -2.27974700 & -2.57554600 & -0.15045000 \\ \mathrm{H} & -1.21643300 & -2.36234000 & -0.30803200 \\ \mathrm{C} & -2.45939800 & -3.00538000 & 1.31263100 \\ \mathrm{H} & -1.84926700 & -3.88826800 & 1.52635100 \\ \mathrm{H} & -2.15415500 & -2.21241100 & 1.99978400 \\ \mathrm{H} & -3.50970100 & -3.25044900 & 1.50672100 \\ \mathrm{C} & -2.66192700 & -3.71719800 & -1.09190900 \\ \mathrm{H} & -2.59168000 & -3.41686700 & -2.14187500 \\ \mathrm{H} & -1.98855600 & -4.56404600 & -0.93229400 \\ \mathrm{H} & -3.68154700 & -4.07000900 & -0.90106600\end{array}$




$\begin{array}{lrrr}\mathrm{C} & 2.29579000 & 0.24509800 & 0.28360300 \\ \mathrm{H} & 3.03518100 & 0.54824700 & 1.03093400 \\ \mathrm{~N} & 2.23257800 & 1.32747800 & -0.66646800 \\ \mathrm{H} & 1.65859200 & 1.23005600 & -1.49254200 \\ \mathrm{C} & 2.77439000 & 2.54614000 & -0.38741500 \\ \mathrm{O} & 3.37811600 & 2.83491200 & 0.62392500 \\ \mathrm{O} & 2.53495000 & 3.40005900 & -1.40198400 \\ \mathrm{C} & 3.06977900 & 4.70691700 & -1.20588000 \\ \mathrm{H} & 4.15487800 & 4.66333400 & -1.09349800 \\ \mathrm{H} & 2.79892600 & 5.27076400 & -2.09714800 \\ \mathrm{H} & 2.63877600 & 5.16705200 & -0.31408400 \\ \mathrm{C} & 2.74770000 & -1.07728600 & -0.31536700 \\ \mathrm{C} & 2.42591900 & -1.47197600 & -1.61521600 \\ \mathrm{C} & 3.52399500 & -1.92697700 & 0.47734200 \\ \mathrm{C} & 2.86942200 & -2.69565400 & -2.10735800 \\ \mathrm{H} & 1.82059700 & -0.83335700 & -2.24831100 \\ \mathrm{C} & 3.95989100 & -3.15415000 & -0.01202000 \\ \mathrm{H} & 3.78713000 & -1.62587600 & 1.48852600 \\ \mathrm{C} & 3.63385200 & -3.54126600 & -1.30801100 \\ \mathrm{H} & 2.61341100 & -2.98850200 & -3.12081000 \\ \mathrm{H} & 4.56050200 & -3.80176400 & 0.61869800 \\ \mathrm{H} & 3.97661800 & -4.49564200 & -1.69542200\end{array}$

\section{Structure anti-product}

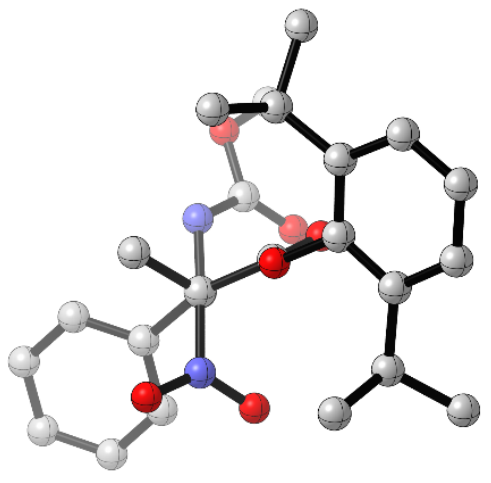

- Thermochemistry -

Zero-point correction $=$

0.520522 (Hartree/Particle)

Thermal correction to Energy=

0.552632

Thermal correction to Enthalpy=

0.553576

Thermal correction to Gibbs Free Energy=

0.454949

Sum of electronic and zero-point Energies=

$-1492.274514$ 
Sum of electronic and thermal Energies=

Sum of electronic and thermal Enthalpies=

Sum of electronic and thermal Free Energies=
$-1492.242403$

$-1492.241459$

$-1492.340087$

Number of Imaginary Frequencies $=0$

E (Single Point Energy) $\left[\mathrm{SMD}_{\text {(Toluene) }} \mathrm{M} 06-2 \mathrm{X} / 6-311++\mathrm{G}(2 \mathrm{~d}, 2 \mathrm{p})\right]=-1493.302498$

$\begin{array}{lrrr}\mathrm{C} & 0.45962200 & 0.10140500 & -0.08850400 \\ \mathrm{O} & 0.57652400 & 0.81426300 & -1.04058200 \\ \mathrm{O} & 1.47675800 & -0.45763100 & 0.60168300 \\ \mathrm{C} & 2.78693900 & -0.21123200 & 0.15275600 \\ \mathrm{C} & 3.45342300 & 0.89485500 & 0.67864500 \\ \mathrm{C} & 3.34445600 & -1.11434100 & -0.75239600 \\ \mathrm{C} & 4.77848500 & 1.07826400 & 0.27992100 \\ \mathrm{C} & 4.67279100 & -0.88865200 & -1.11868900 \\ \mathrm{C} & 5.38232000 & 0.19229500 & -0.60588000 \\ \mathrm{H} & 5.34145400 & 1.92553600 & 0.65728600 \\ \mathrm{H} & 5.15546200 & -1.55913400 & -1.82167800 \\ \mathrm{H} & 6.41340800 & 0.35057000 & -0.90631200 \\ \mathrm{C} & -0.86593100 & -0.31362500 & 0.56781600 \\ \mathrm{C} & -0.91011300 & 0.10546900 & 2.02793400 \\ \mathrm{H} & -0.10357600 & -0.37381200 & 2.58305000 \\ \mathrm{H} & -0.77871500 & 1.18983800 & 2.09235300 \\ \mathrm{H} & -1.86272300 & -0.18028300 & 2.47853700 \\ \mathrm{~N} & -0.87663800 & -1.83349800 & 0.47211100 \\ \mathrm{O} & -0.68661600 & -2.28926600 & -0.64341700 \\ \mathrm{O} & -1.09402500 & -2.48084800 & 1.46937700 \\ \mathrm{C} & 2.51639800 & -2.27483100 & -1.27530400 \\ \mathrm{H} & 1.53455500 & -1.87438600 & -1.55631000 \\ \mathrm{C} & 2.29574400 & -3.32859400 & -0.17965300 \\ \mathrm{H} & 1.83758500 & -2.89677200 & 0.71420100 \\ \mathrm{H} & 1.63626800 & -4.12090500 & -0.54643000 \\ \mathrm{H} & 3.25283700 & -3.77738100 & 0.10891600 \\ \mathrm{C} & 3.11639500 & -2.92712900 & -2.52005300 \\ \mathrm{H} & 3.31035100 & -2.19364100 & -3.30850500 \\ \mathrm{H} & 4.05578300 & -3.44138100 & -2.28764400 \\ \mathrm{H} & 2.42186100 & -3.67510400 & -2.91261100 \\ \mathrm{C} & 2.74765100 & 1.80834800 & 1.66482900 \\ \mathrm{H} & 1.72097900 & 1.95145100 & 1.30159200 \\ \mathrm{C} & 2.68057800 & 1.14245200 & 3.04663600 \\ & & & \\ & & & \end{array}$




$\begin{array}{lrrr}\mathrm{H} & 2.10536900 & 1.75834300 & 3.74638500 \\ \mathrm{H} & 2.21846600 & 0.15305000 & 2.99077700 \\ \mathrm{H} & 3.69101100 & 1.01953300 & 3.45128000 \\ \mathrm{C} & 3.38723400 & 3.19236100 & 1.77103900 \\ \mathrm{H} & 3.49041000 & 3.66410200 & 0.78952100 \\ \mathrm{H} & 2.76960800 & 3.83961800 & 2.40071300 \\ \mathrm{H} & 4.37857900 & 3.13780800 & 2.23359000 \\ \mathrm{C} & -2.06588300 & 0.19468500 & -0.28394600 \\ \mathrm{H} & -1.83844000 & -0.04283700 & -1.32665500 \\ \mathrm{~N} & -2.10181900 & 1.63422400 & -0.21451000 \\ \mathrm{H} & -2.35703800 & 2.10604000 & 0.64059700 \\ \mathrm{C} & -2.11783700 & 2.38690400 & -1.35904500 \\ \mathrm{O} & -2.02751600 & 1.96292500 & -2.48545500 \\ \mathrm{O} & -2.24672300 & 3.69101500 & -1.03676700 \\ \mathrm{C} & -2.25599900 & 4.56698800 & -2.16154100 \\ \mathrm{H} & -3.09277800 & 4.33180500 & -2.82259900 \\ \mathrm{H} & -2.36163000 & 5.57046200 & -1.75171900 \\ \mathrm{H} & -1.32368600 & 4.47590500 & -2.72226200 \\ \mathrm{C} & -3.37207100 & -0.47782000 & 0.11186700 \\ \mathrm{C} & -4.19322300 & 0.04223600 & 1.11480600 \\ \mathrm{C} & -3.75870100 & -1.65600200 & -0.53154000 \\ \mathrm{C} & -5.37109400 & -0.60502000 & 1.47538100 \\ \mathrm{H} & -3.92807500 & 0.96951000 & 1.61686000 \\ \mathrm{C} & -4.93548400 & -2.30572900 & -0.17108600 \\ \mathrm{H} & -3.13303800 & -2.06435000 & -1.32030700 \\ \mathrm{C} & -5.74361300 & -1.78331100 & 0.83431800 \\ \mathrm{H} & -5.99904100 & -0.18556600 & 2.25512000 \\ \mathrm{H} & -5.22276000 & -3.21856200 & -0.68309600 \\ \mathrm{H} & -6.66302300 & -2.28806100 & 1.11356900\end{array}$

\section{Structure anti-(2R,3R)-TS}

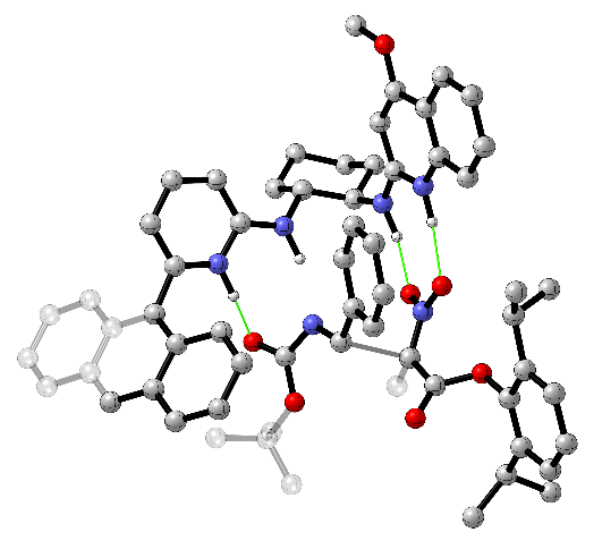

- Thermochemistry - 
Zero-point correction $=$

Thermal correction to Energy=

Thermal correction to Enthalpy=

Thermal correction to Gibbs Free Energy=

Sum of electronic and zero-point Energies=

Sum of electronic and thermal Energies=

Sum of electronic and thermal Enthalpies=

Sum of electronic and thermal Free Energies=
1.223288 (Hartree/Particle)

1.291543

1.292487

1.117935

$-3256.481416$

$-3256.413161$

$-3256.412217$

$-3256.586769$

Number of Imaginary Frequencies $=1$

E (Single Point Energy) [SMD(Toluene) M06-2X/6-311++G(2d,2p)] = -3258.781514

$\begin{array}{llll}\mathrm{O} & -2.43023500 & -3.32394600 & 0.13436000 \\ \mathrm{C} & -2.76324300 & -2.32465700 & 0.72671500 \\ \mathrm{O} & -4.03647200 & -1.93208600 & 0.89652100 \\ \mathrm{C} & -1.76764400 & -1.47831300 & 1.44630800 \\ \mathrm{C} & -1.09482300 & -2.13981200 & 2.61606000 \\ \mathrm{H} & -0.90415400 & -3.18547800 & 2.37056700 \\ \mathrm{H} & -1.73207800 & -2.08222500 & 3.50610500 \\ \mathrm{H} & -0.15227500 & -1.63903500 & 2.84467200 \\ \mathrm{~N} & -2.01873900 & -0.13248700 & 1.59624400 \\ \mathrm{O} & -2.75875200 & 0.48382900 & 0.79725600 \\ \mathrm{O} & -1.35892800 & 0.48055400 & 2.46827100 \\ \mathrm{C} & -0.17885100 & -1.26370700 & 0.00776600 \\ \mathrm{C} & -0.82501100 & -0.47129700 & -1.06547000 \\ \mathrm{C} & -1.69962400 & -1.08795700 & -1.97049300 \\ \mathrm{C} & -0.50966300 & 0.88216200 & -1.23830700\end{array}$




\begin{tabular}{|c|c|c|c|}
\hline $\mathrm{C}$ & -2.25264800 & -0.36068600 & -3.02057900 \\
\hline $\mathrm{C}$ & -1.05936700 & 1.60318900 & -2.29250100 \\
\hline $\mathrm{C}$ & -1.93839500 & 0.98738200 & -3.18232100 \\
\hline $\mathrm{C}$ & 1.80653100 & -1.56369100 & 1.15900900 \\
\hline $\mathrm{O}$ & 2.91880800 & -1.12836400 & 1.50014800 \\
\hline $\mathrm{O}$ & 1.47712300 & -2.84716700 & 1.26282000 \\
\hline $\mathrm{C}$ & 2.40160100 & -3.84875500 & 1.79805200 \\
\hline $\mathrm{C}$ & 2.77462600 & -3.50200000 & 3.23541100 \\
\hline $\mathrm{H}$ & 3.40485900 & -2.61375800 & 3.28139400 \\
\hline $\mathrm{H}$ & 3.31651800 & -4.34413700 & 3.67632800 \\
\hline $\mathrm{H}$ & 1.86897500 & -3.33443600 & 3.82779700 \\
\hline $\mathrm{C}$ & 3.61262300 & -3.97013800 & 0.88214500 \\
\hline $\mathrm{H}$ & 3.28058600 & -4.19804500 & -0.13501900 \\
\hline $\mathrm{H}$ & 4.24756400 & -4.79123300 & 1.22972500 \\
\hline $\mathrm{H}$ & 4.20275200 & -3.05121400 & 0.87051700 \\
\hline $\mathrm{C}$ & 1.56853500 & -5.12454700 & 1.75170700 \\
\hline $\mathrm{H}$ & 2.17135300 & -5.97383800 & 2.08475300 \\
\hline $\mathrm{H}$ & 1.22250400 & -5.31668600 & 0.73220700 \\
\hline $\mathrm{H}$ & 0.69627000 & -5.04177600 & 2.40674000 \\
\hline $\mathrm{N}$ & 0.87718600 & -0.71908000 & 0.62096900 \\
\hline $\mathrm{H}$ & -0.24113500 & -2.34768700 & -0.11295800 \\
\hline $\mathrm{H}$ & -2.36824200 & 1.55421600 & -4.00284700 \\
\hline $\mathrm{H}$ & -2.92409400 & -0.85103300 & -3.71848400 \\
\hline $\mathrm{H}$ & -0.80337800 & 2.65198500 & -2.42269400 \\
\hline $\mathrm{H}$ & 0.18454900 & 1.35192700 & -0.54601600 \\
\hline $\mathrm{H}$ & -1.93900400 & -2.14109100 & -1.85306400 \\
\hline $\mathrm{C}$ & 1.49074500 & 4.59643000 & 4.54487200 \\
\hline $\mathrm{C}$ & 2.79584800 & 4.76701500 & 3.76785500 \\
\hline
\end{tabular}




\begin{tabular}{|c|c|c|c|}
\hline $\mathrm{C}$ & 3.03508700 & 3.56199100 & 2.85901700 \\
\hline $\mathrm{C}$ & 1.87672100 & 3.34565400 & 1.87407000 \\
\hline $\mathrm{C}$ & 0.54467700 & 3.23568300 & 2.63119000 \\
\hline $\mathrm{C}$ & 0.31401500 & 4.41776800 & 3.58467700 \\
\hline $\mathrm{H}$ & 3.13470400 & 2.65160300 & 3.46466200 \\
\hline $\mathrm{H}$ & 3.64051700 & 4.88808200 & 4.45194700 \\
\hline $\mathrm{H}$ & 2.74543900 & 5.68354700 & 3.16275700 \\
\hline $\mathrm{H}$ & 1.30894500 & 5.45789000 & 5.19388000 \\
\hline $\mathrm{H}$ & 1.56835400 & 3.71738300 & 5.19761600 \\
\hline $\mathrm{H}$ & 1.81280400 & 4.20878200 & 1.19751500 \\
\hline $\mathrm{H}$ & -0.61790900 & 4.23617500 & 4.12832300 \\
\hline $\mathrm{H}$ & 0.17254900 & 5.34596800 & 3.01839100 \\
\hline $\mathrm{H}$ & 3.96928700 & 3.67175700 & 2.29646300 \\
\hline $\mathrm{N}$ & 2.08963200 & 2.14642500 & 1.06757100 \\
\hline $\mathrm{H}$ & 1.62789800 & 1.26284200 & 1.31603000 \\
\hline $\mathrm{N}$ & -0.58677900 & 2.96487100 & 1.75390500 \\
\hline $\mathrm{H}$ & -1.02362400 & 2.04316600 & 1.92859400 \\
\hline $\mathrm{C}$ & -1.15990500 & 3.73530200 & 0.82962700 \\
\hline $\mathrm{C}$ & -0.74908100 & 5.05086900 & 0.46712400 \\
\hline $\mathrm{C}$ & -2.91444300 & 3.82796900 & -0.81192000 \\
\hline $\mathrm{H}$ & -2.47529900 & 2.22016100 & 0.43044600 \\
\hline $\mathrm{C}$ & -1.44549900 & 5.73731600 & -0.49927600 \\
\hline $\mathrm{H}$ & 0.09057100 & 5.49534700 & 0.97600000 \\
\hline $\mathrm{C}$ & -3.95203500 & 3.15161600 & -1.47015600 \\
\hline $\mathrm{C}$ & -2.56319300 & 5.13478000 & -1.18268400 \\
\hline $\mathrm{O}$ & -1.15788300 & 6.97588300 & -0.89082400 \\
\hline $\mathrm{C}$ & -4.65049300 & 3.80578900 & -2.46362700 \\
\hline $\mathrm{H}$ & -4.16926700 & 2.12295400 & -1.19526700 \\
\hline
\end{tabular}




\begin{tabular}{|c|c|c|c|}
\hline $\mathrm{C}$ & -3.28846800 & 5.78299500 & -2.20096800 \\
\hline $\mathrm{C}$ & -4.32545500 & 5.12636300 & -2.82837700 \\
\hline $\mathrm{H}$ & -5.46048600 & 3.29128600 & -2.97146900 \\
\hline $\mathrm{H}$ & -3.01549200 & 6.79503100 & -2.47691100 \\
\hline $\mathrm{H}$ & -4.88896300 & 5.62385200 & -3.60971700 \\
\hline $\mathrm{N}$ & -2.21602800 & 3.19431200 & 0.19129200 \\
\hline $\mathrm{C}$ & 3.07788400 & 2.06970500 & 0.15292100 \\
\hline $\mathrm{C}$ & 3.66062700 & 3.18173500 & -0.49229700 \\
\hline $\mathrm{C}$ & 4.48298400 & 0.60182000 & -1.11893900 \\
\hline $\mathrm{C}$ & 4.62091500 & 2.96665200 & -1.45680600 \\
\hline $\mathrm{H}$ & 3.33441100 & 4.18318200 & -0.24560100 \\
\hline $\mathrm{C}$ & 5.03296400 & 1.67073300 & -1.79581500 \\
\hline $\mathrm{H}$ & 5.05617900 & 3.81817700 & -1.97050200 \\
\hline $\mathrm{H}$ & 5.77871500 & 1.48849400 & -2.55932300 \\
\hline $\mathrm{N}$ & 3.54292200 & 0.83876800 & -0.16766300 \\
\hline $\mathrm{C}$ & 4.88480600 & -0.80325500 & -1.37203000 \\
\hline $\mathrm{C}$ & 6.22145800 & -1.18146500 & -1.12364500 \\
\hline $\mathrm{C}$ & 3.95323700 & -1.70809100 & -1.92414500 \\
\hline $\mathrm{C}$ & 7.18433800 & -0.31807400 & -0.50616200 \\
\hline $\mathrm{C}$ & 6.64266900 & -2.50876000 & -1.47406500 \\
\hline $\mathrm{C}$ & 4.40220100 & -3.02429000 & -2.28398500 \\
\hline $\mathrm{C}$ & 2.58282200 & -1.37586400 & -2.18053300 \\
\hline $\mathrm{C}$ & 8.46315900 & -0.73709500 & -0.28286000 \\
\hline $\mathrm{H}$ & 6.88610000 & 0.67739900 & -0.19552600 \\
\hline $\mathrm{C}$ & 7.99447300 & -2.90594800 & -1.22741900 \\
\hline $\mathrm{C}$ & 5.72830400 & -3.38732200 & -2.05350600 \\
\hline $\mathrm{C}$ & 3.47800700 & -3.94952000 & -2.86320200 \\
\hline $\mathrm{H}$ & 2.20501400 & -0.38787200 & -1.93459800 \\
\hline
\end{tabular}




\begin{tabular}{|c|c|c|c|}
\hline $\mathrm{C}$ & 1.73080900 & -2.29203400 & -2.72733700 \\
\hline $\mathrm{C}$ & 8.88263100 & -2.04768400 & -0.65431300 \\
\hline $\mathrm{H}$ & 9.17272100 & -0.06742300 & 0.19265400 \\
\hline $\mathrm{H}$ & 8.29210600 & -3.91248200 & -1.50753700 \\
\hline $\mathrm{H}$ & 6.05780700 & -4.38667000 & -2.32968200 \\
\hline $\mathrm{C}$ & 2.17789800 & -3.60069400 & -3.07183500 \\
\hline $\mathrm{H}$ & 3.84077400 & -4.93926700 & -3.12677200 \\
\hline $\mathrm{H}$ & 0.69780900 & -2.01351000 & -2.91330300 \\
\hline $\mathrm{H}$ & 9.90612400 & -2.35587600 & -0.46935700 \\
\hline $\mathrm{H}$ & 1.48041000 & -4.30798600 & -3.50807500 \\
\hline $\mathrm{C}$ & -7.04198800 & -4.02080800 & -1.06024500 \\
\hline $\mathrm{C}$ & -6.36904100 & -4.62606400 & -0.00803900 \\
\hline $\mathrm{C}$ & -5.34700800 & -3.96199100 & 0.67776700 \\
\hline $\mathrm{C}$ & -5.03450100 & -2.67551500 & 0.24206700 \\
\hline $\mathrm{C}$ & -5.70403200 & -2.01913100 & -0.79509800 \\
\hline $\mathrm{C}$ & -6.71891100 & -2.72168400 & -1.44307500 \\
\hline $\mathrm{H}$ & -7.83059900 & -4.55683900 & -1.57861900 \\
\hline $\mathrm{H}$ & -6.63926600 & -5.63428200 & 0.29398300 \\
\hline $\mathrm{C}$ & -4.65689900 & -4.62715100 & 1.85512700 \\
\hline $\mathrm{C}$ & -5.38215800 & -0.56840300 & -1.10596100 \\
\hline $\mathrm{H}$ & -7.26563400 & -2.25165600 & -2.25432400 \\
\hline $\mathrm{C}$ & -5.66775100 & -4.95810100 & 2.96035600 \\
\hline $\mathrm{H}$ & -3.93446400 & -3.92065200 & 2.27762000 \\
\hline $\mathrm{C}$ & -3.89419200 & -5.88136200 & 1.41480700 \\
\hline $\mathrm{H}$ & -3.36886100 & -6.32479300 & 2.26707900 \\
\hline $\mathrm{H}$ & -3.16605500 & -5.63940400 & 0.63748300 \\
\hline $\mathrm{H}$ & -4.58481300 & -6.63524900 & 1.02146800 \\
\hline H & -6.40381300 & -5.69111300 & 2.61431500 \\
\hline
\end{tabular}




$\begin{array}{llll}\mathrm{H} & -6.20866900 & -4.06462000 & 3.28479400 \\ \mathrm{H} & -5.15456100 & -5.38675700 & 3.82693200 \\ \mathrm{H} & -4.29740500 & -0.43389800 & -1.01053300 \\ \mathrm{C} & -6.05843800 & 0.31978700 & -0.04914500 \\ \mathrm{C} & -5.79484400 & -0.15073700 & -2.51764600 \\ \mathrm{H} & -5.92266200 & 1.38386800 & -0.27271600 \\ \mathrm{H} & -5.64344500 & 0.12254900 & 0.94276200 \\ \mathrm{H} & -7.13538400 & 0.12160600 & -0.02472000 \\ \mathrm{H} & -6.88285300 & -0.06420500 & -2.61162000 \\ \mathrm{H} & -5.44558700 & -0.86500500 & -3.27001400 \\ \mathrm{H} & -5.36473200 & 0.82793500 & -2.76096800 \\ \mathrm{C} & -0.08801800 & 7.66347300 & -0.25698700 \\ \mathrm{H} & -0.05309600 & 8.64798300 & -0.71877700 \\ \mathrm{H} & 0.85896800 & 7.14025200 & -0.42633900 \\ \mathrm{H} & -0.27750000 & 7.76186000 & 0.81667400 \\ \mathrm{H} & 0.61060600 & 2.32955100 & 3.24964400 \\ \mathrm{H} & 3.23798200 & 0.02968200 & 0.45257700\end{array}$

\section{Structure syn-(2S,3R)-TS}

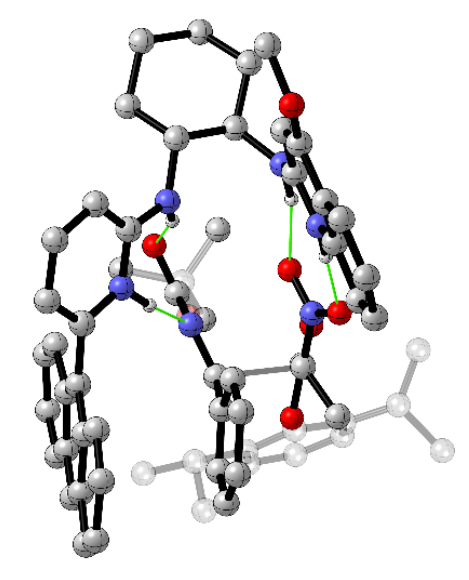

- Thermochemistry - 
Zero-point correction $=$

Thermal correction to Energy=

Thermal correction to Enthalpy=

Thermal correction to Gibbs Free Energy=

Sum of electronic and zero-point Energies=

Sum of electronic and thermal Energies $=$

Sum of electronic and thermal Enthalpies=

Sum of electronic and thermal Free Energies=
1.224025 (Hartree/Particle)

1.291372

1.292316

1.123332

$-3256.497019$

$-3256.429673$

$-3256.428728$

$-3256.597713$

Number of Imaginary Frequencies $=1$

E (Single Point Energy) $\left[\operatorname{SMD}_{\text {(Toluene) }}\right.$ M06-2X/6-311++G(2d,2p) $]=-3258.790148$

$\begin{array}{llll}\mathrm{O} & -2.79527700 & -2.67566400 & 0.73456000 \\ \mathrm{C} & -2.72669100 & -1.56651600 & 1.18942400 \\ \mathrm{O} & -3.78722200 & -0.73997900 & 1.37828700 \\ \mathrm{C} & -1.51819700 & -0.86499700 & 1.72573000 \\ \mathrm{C} & -1.70752600 & -0.22828800 & 3.08379900 \\ \mathrm{H} & -2.56248700 & 0.44758000 & 3.04831300 \\ \mathrm{H} & -0.82107800 & 0.33167500 & 3.37928600 \\ \mathrm{H} & -1.90092900 & -0.99444500 & 3.84390700 \\ \mathrm{~N} & -0.32253800 & -1.57569300 & 1.58410300 \\ \mathrm{O} & -0.09207900 & -2.21306700 & 0.55281000 \\ \mathrm{O} & 0.56029200 & -1.41966100 & 2.46431200 \\ \mathrm{C} & -1.14905500 & 0.60922500 & 0.29783700 \\ \mathrm{C} & -0.52677100 & 1.63589100 & 1.16942300 \\ \mathrm{C} & -1.30373000 & 2.65849200 & 1.71282000 \\ \mathrm{C} & 0.83384700 & 1.55240600 & 1.50224200\end{array}$




\begin{tabular}{|c|c|c|c|}
\hline $\mathrm{C}$ & -0.73328900 & 3.59292700 & 2.57518500 \\
\hline $\mathrm{C}$ & 1.39824000 & 2.47985600 & 2.36815400 \\
\hline $\mathrm{C}$ & 0.61436700 & 3.50320600 & 2.90586400 \\
\hline $\mathrm{C}$ & -0.82743400 & -0.69980300 & -1.65768300 \\
\hline $\mathrm{O}$ & -0.04941800 & -1.12315800 & -2.52014400 \\
\hline $\mathrm{O}$ & -2.12344700 & -1.00312700 & -1.63168000 \\
\hline $\mathrm{C}$ & -2.64764200 & -2.04056600 & -2.54359500 \\
\hline $\mathrm{C}$ & -1.89456200 & -3.35140000 & -2.32726100 \\
\hline $\mathrm{H}$ & -0.89808100 & -3.31878400 & -2.76960300 \\
\hline $\mathrm{H}$ & -2.45997900 & -4.16138300 & -2.79824700 \\
\hline $\mathrm{H}$ & -1.81847800 & -3.55639400 & -1.25588400 \\
\hline $\mathrm{C}$ & -2.57457100 & -1.54750900 & -3.98489700 \\
\hline $\mathrm{H}$ & -3.01220700 & -2.30772100 & -4.63945500 \\
\hline $\mathrm{H}$ & -1.54549000 & -1.36498600 & -4.29610700 \\
\hline $\mathrm{H}$ & -3.16239800 & -0.62999900 & -4.09421800 \\
\hline $\mathrm{C}$ & -4.10567400 & -2.19715000 & -2.13462300 \\
\hline $\mathrm{H}$ & -4.58611100 & -2.91681700 & -2.80421900 \\
\hline $\mathrm{H}$ & -4.63476900 & -1.24436100 & -2.22672300 \\
\hline $\mathrm{H}$ & -4.19472200 & -2.56025200 & -1.10906700 \\
\hline $\mathrm{N}$ & -0.35816400 & 0.13018500 & -0.67516400 \\
\hline $\mathrm{H}$ & -2.22343600 & 0.72131000 & 0.12278800 \\
\hline $\mathrm{H}$ & 1.05922000 & 4.23552400 & 3.57243500 \\
\hline $\mathrm{H}$ & -1.34117500 & 4.39412700 & 2.98371500 \\
\hline $\mathrm{H}$ & 2.45096000 & 2.40759500 & 2.62731600 \\
\hline $\mathrm{H}$ & 1.43089100 & 0.73972300 & 1.09502100 \\
\hline $\mathrm{H}$ & -2.35802800 & 2.72110600 & 1.45469200 \\
\hline $\mathrm{C}$ & 3.57904700 & -4.69352400 & -3.34200400 \\
\hline $\mathrm{C}$ & 4.08445700 & -3.46423200 & -4.10130100 \\
\hline
\end{tabular}




\begin{tabular}{|c|c|c|c|}
\hline $\mathrm{C}$ & 3.30303000 & -2.20996300 & -3.70243600 \\
\hline $\mathrm{C}$ & 3.38013200 & -1.96977300 & -2.18922500 \\
\hline $\mathrm{C}$ & 2.87080500 & -3.21901300 & -1.44082500 \\
\hline $\mathrm{C}$ & 3.65343500 & -4.47638500 & -1.82722400 \\
\hline $\mathrm{H}$ & 2.24429100 & -2.31563500 & -3.97441500 \\
\hline $\mathrm{H}$ & 4.00582600 & -3.62363800 & -5.18053500 \\
\hline $\mathrm{H}$ & 5.15137500 & -3.31498400 & -3.88212200 \\
\hline $\mathrm{H}$ & 4.15752100 & -5.57986200 & -3.61795300 \\
\hline $\mathrm{H}$ & 2.53759500 & -4.89283900 & -3.62628300 \\
\hline $\mathrm{H}$ & 4.42264000 & -1.77751000 & -1.90369500 \\
\hline $\mathrm{H}$ & 3.24130100 & -5.33065100 & -1.28245700 \\
\hline $\mathrm{H}$ & 4.70628500 & -4.38614800 & -1.53026800 \\
\hline $\mathrm{H}$ & 3.68005200 & -1.32671600 & -4.23081800 \\
\hline $\mathrm{N}$ & 2.56610300 & -0.85094000 & -1.74728700 \\
\hline $\mathrm{H}$ & 1.54766300 & -1.02965400 & -1.81895100 \\
\hline $\mathrm{N}$ & 2.73673500 & -2.98809700 & 0.00385100 \\
\hline $\mathrm{H}$ & 1.76699800 & -2.83794800 & 0.29666700 \\
\hline $\mathrm{C}$ & 3.64484800 & -2.36625000 & 0.77558200 \\
\hline $\mathrm{C}$ & 5.04819400 & -2.39798700 & 0.54963200 \\
\hline $\mathrm{C}$ & 3.98107300 & -1.01664200 & 2.73233000 \\
\hline $\mathrm{H}$ & 2.14939900 & -1.66416200 & 2.00419200 \\
\hline $\mathrm{C}$ & 5.89094200 & -1.74336900 & 1.41710600 \\
\hline $\mathrm{H}$ & 5.42642800 & -2.98067600 & -0.27485000 \\
\hline $\mathrm{C}$ & 3.39621700 & -0.31453000 & 3.79917800 \\
\hline $\mathrm{C}$ & 5.37245800 & -1.01103300 & 2.54545900 \\
\hline $\mathrm{O}$ & 7.21594600 & -1.72697900 & 1.30438500 \\
\hline $\mathrm{C}$ & 4.21721700 & 0.37289900 & 4.66963800 \\
\hline $\mathrm{H}$ & 2.31558100 & -0.31940600 & 3.91287900 \\
\hline
\end{tabular}




\begin{tabular}{|c|c|c|c|}
\hline $\mathrm{C}$ & 6.18891900 & -0.30738700 & 3.45132300 \\
\hline $\mathrm{C}$ & 5.61508200 & 0.37559300 & 4.50197400 \\
\hline $\mathrm{H}$ & 3.77538000 & 0.91726900 & 5.49793000 \\
\hline $\mathrm{H}$ & 7.26295200 & -0.31553100 & 3.30531000 \\
\hline $\mathrm{H}$ & 6.24042400 & 0.91737700 & 5.20275200 \\
\hline $\mathrm{N}$ & 3.17933500 & -1.69978000 & 1.84257100 \\
\hline $\mathrm{C}$ & 2.88908600 & 0.44401000 & -1.91580100 \\
\hline $\mathrm{C}$ & 4.12894900 & 0.94881300 & -2.36150300 \\
\hline $\mathrm{C}$ & 2.02548400 & 2.67501200 & -1.75294100 \\
\hline $\mathrm{C}$ & 4.27173600 & 2.31259800 & -2.50510100 \\
\hline $\mathrm{H}$ & 4.92863400 & 0.26702900 & -2.62167200 \\
\hline $\mathrm{C}$ & 3.21820100 & 3.19381100 & -2.21814500 \\
\hline $\mathrm{H}$ & 5.21169400 & 2.71043900 & -2.87506200 \\
\hline $\mathrm{H}$ & 3.31293200 & 4.26128600 & -2.37236300 \\
\hline $\mathrm{N}$ & 1.91373100 & 1.33092900 & -1.59838400 \\
\hline $\mathrm{C}$ & 0.81897300 & 3.47583200 & -1.43882900 \\
\hline $\mathrm{C}$ & -0.39381000 & 3.15230900 & -2.08839000 \\
\hline $\mathrm{C}$ & 0.89296600 & 4.54144200 & -0.51435900 \\
\hline $\mathrm{C}$ & -0.49616100 & 2.16542600 & -3.12326200 \\
\hline $\mathrm{C}$ & -1.58398500 & 3.87502500 & -1.74006900 \\
\hline $\mathrm{C}$ & -0.29862400 & 5.29088000 & -0.22820700 \\
\hline $\mathrm{C}$ & 2.09065300 & 4.91321600 & 0.17838500 \\
\hline $\mathrm{C}$ & -1.69194400 & 1.88379500 & -3.71344000 \\
\hline $\mathrm{H}$ & 0.38615000 & 1.63065700 & -3.45561900 \\
\hline $\mathrm{C}$ & -2.82511700 & 3.53148600 & -2.36623900 \\
\hline $\mathrm{C}$ & -1.50459500 & 4.92388700 & -0.82476900 \\
\hline $\mathrm{C}$ & -0.23769500 & 6.38957400 & 0.68455400 \\
\hline $\mathrm{H}$ & 2.99326800 & 4.33085900 & 0.03198000 \\
\hline
\end{tabular}




\begin{tabular}{|c|c|c|c|}
\hline $\mathrm{C}$ & 2.11064100 & 5.97614000 & 1.03439800 \\
\hline $\mathrm{C}$ & -2.88271300 & 2.55941500 & -3.31931700 \\
\hline $\mathrm{H}$ & -1.73306600 & 1.14206100 & -4.50345500 \\
\hline $\mathrm{H}$ & -3.71495600 & 4.08703700 & -2.08000000 \\
\hline $\mathrm{H}$ & -2.40656800 & 5.48160700 & -0.58000700 \\
\hline $\mathrm{C}$ & 0.93311400 & 6.73488100 & 1.28997400 \\
\hline $\mathrm{H}$ & -1.15095700 & 6.94587600 & 0.87808300 \\
\hline $\mathrm{H}$ & 3.03401300 & 6.24075000 & 1.54045600 \\
\hline $\mathrm{H}$ & -3.82259500 & 2.31349000 & -3.80328400 \\
\hline $\mathrm{H}$ & 0.97334700 & 7.57755900 & 1.97233900 \\
\hline $\mathrm{C}$ & -7.66467600 & -1.93127100 & 0.50848300 \\
\hline $\mathrm{C}$ & -6.94240500 & -2.62461000 & 1.46699900 \\
\hline $\mathrm{C}$ & -5.63428700 & -2.25571300 & 1.80127600 \\
\hline $\mathrm{C}$ & -5.08632200 & -1.18237800 & 1.09894500 \\
\hline $\mathrm{C}$ & -5.79850600 & -0.42419200 & 0.15797000 \\
\hline $\mathrm{C}$ & -7.10083600 & -0.82783500 & -0.12799900 \\
\hline $\mathrm{H}$ & -8.67703800 & -2.23689300 & 0.26472100 \\
\hline $\mathrm{H}$ & -7.39936800 & -3.46629100 & 1.98013100 \\
\hline $\mathrm{C}$ & -4.91195800 & -2.98439700 & 2.92132400 \\
\hline $\mathrm{C}$ & -5.15718100 & 0.79789700 & -0.48140100 \\
\hline $\mathrm{H}$ & -7.68629700 & -0.28238900 & -0.85970800 \\
\hline $\mathrm{C}$ & -5.68480700 & -2.84432800 & 4.23946500 \\
\hline $\mathrm{H}$ & -3.93416100 & -2.51650300 & 3.07297400 \\
\hline $\mathrm{C}$ & -4.68346300 & -4.45892200 & 2.57320200 \\
\hline $\mathrm{H}$ & -4.13107500 & -4.95750500 & 3.37607200 \\
\hline $\mathrm{H}$ & -4.11770400 & -4.55580100 & 1.64421200 \\
\hline $\mathrm{H}$ & -5.63982700 & -4.97968300 & 2.45453900 \\
\hline $\mathrm{H}$ & -6.66439400 & -3.32901800 & 4.17667300 \\
\hline
\end{tabular}




$\begin{array}{llll}\mathrm{H} & -5.84517600 & -1.79332700 & 4.49670700 \\ \mathrm{H} & -5.13051100 & -3.32053600 & 5.05438500 \\ \mathrm{H} & -4.16118700 & 0.50043600 & -0.84055300 \\ \mathrm{C} & -4.97821800 & 1.92042200 & 0.55248000 \\ \mathrm{C} & -5.94411500 & 1.33478900 & -1.67638100 \\ \mathrm{H} & -4.43429300 & 2.76185400 & 0.10478200 \\ \mathrm{H} & -4.42564900 & 1.57538700 & 1.43078300 \\ \mathrm{H} & -5.95462300 & 2.28577500 & 0.88844600 \\ \mathrm{H} & -6.93349800 & 1.69196200 & -1.37163600 \\ \mathrm{H} & -6.08064400 & 0.57836900 & -2.45659200 \\ \mathrm{H} & -5.41228800 & 2.18495600 & -2.11201400 \\ \mathrm{C} & 7.82050300 & -2.44998100 & 0.23932200 \\ \mathrm{H} & 8.89207500 & -2.29624600 & 0.34754500 \\ \mathrm{H} & 7.48455700 & -2.06120100 & -0.72780900 \\ \mathrm{H} & 7.58696400 & -3.51614400 & 0.31823800 \\ \mathrm{H} & 1.83373500 & -3.36899600 & -1.77200900 \\ \mathrm{H} & 0.99196100 & 0.90995700 & -1.19419200\end{array}$

\section{Structure syn-(2R,3S)-TS}

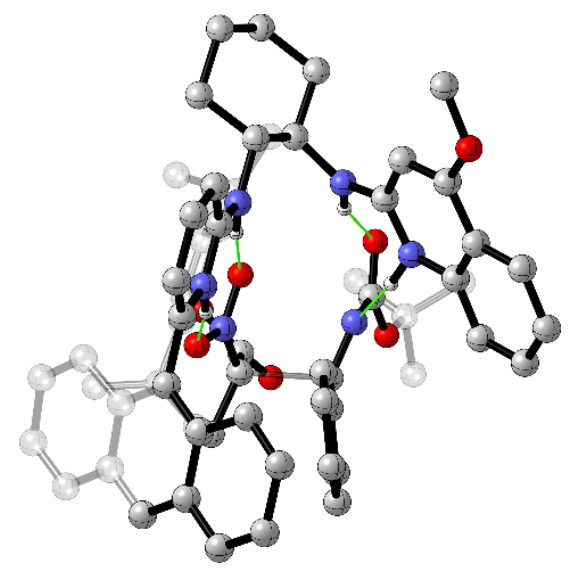

- Thermochemistry - 


$\begin{array}{ll}\text { Zero-point correction= } & 1.223019 \text { (Hartree/Particle) } \\ \text { Thermal correction to Energy= } & 1.291023 \\ \text { Thermal correction to Enthalpy= } & 1.291967 \\ \text { Thermal correction to Gibbs Free Energy= } & 1.120363 \\ \text { Sum of electronic and zero-point Energies= } & -3256.488852 \\ \text { Sum of electronic and thermal Energies }= & -3256.420848 \\ \text { Sum of electronic and thermal Enthalpies }= & -3256.419903 \\ \text { Sum of electronic and thermal Free Energies }= & -3256.591507\end{array}$

Number of Imaginary Frequencies $=1$

E (Single Point Energy) $\left[\operatorname{SMD}_{(\text {Toluene) }}\right.$ M06-2X/6-311++G(2d,2p) $]=-3258.785444$

$\begin{array}{llll}\mathrm{O} & -3.52764600 & 1.62137700 & 1.59568500 \\ \mathrm{C} & -2.86205700 & 1.56683200 & 0.59334300 \\ \mathrm{O} & -3.35827300 & 1.43915200 & -0.65206300 \\ \mathrm{C} & -1.37708000 & 1.78742300 & 0.61797600 \\ \mathrm{C} & -0.99557400 & 3.20309000 & 0.97028200 \\ \mathrm{H} & -1.26596400 & 3.89298200 & 0.16237300 \\ \mathrm{H} & 0.07828300 & 3.28586200 & 1.14155100 \\ \mathrm{H} & -1.53818700 & 3.49327700 & 1.87423400 \\ \mathrm{~N} & -0.65257000 & 1.21257700 & -0.42068000 \\ \mathrm{O} & 0.40967600 & 1.76462600 & -0.78459100 \\ \mathrm{O} & -0.98130500 & 0.09121900 & -0.84948900 \\ \mathrm{C} & -0.79397600 & 0.55190900 & 2.12478500 \\ \mathrm{C} & 0.62726300 & 0.95069400 & 2.29563800 \\ \mathrm{C} & 1.62095900 & 0.36541700 & 1.49920500 \\ \mathrm{C} & 0.99540600 & 1.89531700 & 3.25563300\end{array}$




\begin{tabular}{|c|c|c|c|}
\hline $\mathrm{C}$ & 2.95489200 & 0.70503600 & 1.67599700 \\
\hline $\mathrm{C}$ & 2.33415600 & 2.24257100 & 3.42550600 \\
\hline $\mathrm{C}$ & 3.31653300 & 1.64816400 & 2.63777000 \\
\hline $\mathrm{C}$ & -2.22235800 & -1.32635700 & 1.89962200 \\
\hline $\mathrm{O}$ & -2.51871000 & -2.29749500 & 1.20305300 \\
\hline $\mathrm{O}$ & -2.98296700 & -0.83047200 & 2.86783400 \\
\hline $\mathrm{C}$ & -4.32569300 & -1.34208600 & 3.14939000 \\
\hline $\mathrm{C}$ & -5.22553600 & -1.19462400 & 1.92842300 \\
\hline $\mathrm{H}$ & -6.23268600 & -1.53807000 & 2.18566200 \\
\hline $\mathrm{H}$ & -5.28595900 & -0.14659700 & 1.62142300 \\
\hline $\mathrm{C}$ & -4.79612100 & -0.42459800 & 4.27073900 \\
\hline $\mathrm{H}$ & -4.14080200 & -0.51485600 & 5.14200300 \\
\hline $\mathrm{H}$ & -5.81352500 & -0.69256300 & 4.56888000 \\
\hline $\mathrm{H}$ & -4.78801500 & 0.61366400 & 3.92743500 \\
\hline $\mathrm{C}$ & -4.22491800 & -2.78728300 & 3.62639800 \\
\hline $\mathrm{H}$ & -3.51712500 & -2.86121200 & 4.45798300 \\
\hline $\mathrm{H}$ & -3.90137000 & -3.44639600 & 2.81950700 \\
\hline $\mathrm{H}$ & -5.20523100 & -3.11834400 & 3.98224000 \\
\hline $\mathrm{N}$ & -0.99069800 & -0.72485700 & 1.76240800 \\
\hline $\mathrm{H}$ & -1.52145700 & 1.03381200 & 2.78481400 \\
\hline $\mathrm{H}$ & 4.36111000 & 1.91877600 & 2.76522600 \\
\hline $\mathrm{H}$ & 3.72094800 & 0.23393000 & 1.06603900 \\
\hline $\mathrm{H}$ & 2.60956300 & 2.97449300 & 4.17818000 \\
\hline $\mathrm{H}$ & 0.22861000 & 2.35316200 & 3.87571700 \\
\hline $\mathrm{H}$ & 1.33014900 & -0.35806400 & 0.74050100 \\
\hline $\mathrm{C}$ & -1.26838900 & -4.31826000 & -4.00824000 \\
\hline $\mathrm{C}$ & -0.09095500 & -3.76359500 & -4.81312500 \\
\hline $\mathrm{C}$ & 0.35266100 & -2.40170900 & -4.27328200 \\
\hline
\end{tabular}




\begin{tabular}{|c|c|c|c|}
\hline $\mathrm{C}$ & 0.71639000 & -2.49445500 & -2.78487000 \\
\hline $\mathrm{C}$ & -0.50291100 & -3.01379200 & -1.99581300 \\
\hline $\mathrm{C}$ & -0.92985000 & -4.39239900 & -2.51714100 \\
\hline $\mathrm{H}$ & -0.45519700 & -1.66700500 & -4.38467800 \\
\hline $\mathrm{H}$ & -0.35696300 & -3.67396600 & -5.87022700 \\
\hline $\mathrm{H}$ & 0.75136500 & -4.46786800 & -4.75872300 \\
\hline $\mathrm{H}$ & -1.55238900 & -5.30801700 & -4.37720700 \\
\hline $\mathrm{H}$ & -2.14192200 & -3.66611300 & -4.14559200 \\
\hline $\mathrm{H}$ & 1.54520400 & -3.20510700 & -2.66972800 \\
\hline $\mathrm{H}$ & -1.78858300 & -4.73470900 & -1.93169400 \\
\hline $\mathrm{H}$ & -0.12551800 & -5.12325000 & -2.35668600 \\
\hline $\mathrm{H}$ & 1.20903900 & -2.01710600 & -4.83899700 \\
\hline $\mathrm{N}$ & 1.14661400 & -1.21793600 & -2.22896200 \\
\hline $\mathrm{H}$ & 0.42935300 & -0.65676400 & -1.74458000 \\
\hline $\mathrm{N}$ & -0.35040800 & -2.92930200 & -0.55188900 \\
\hline $\mathrm{H}$ & -1.12671800 & -2.48781700 & -0.03622000 \\
\hline $\mathrm{C}$ & 0.70032000 & -3.29755800 & 0.19235800 \\
\hline $\mathrm{C}$ & 1.71447100 & -4.20679500 & -0.22671500 \\
\hline $\mathrm{C}$ & 1.79226000 & -3.02082300 & 2.30154600 \\
\hline $\mathrm{C}$ & 2.74731200 & -4.50376400 & 0.62940200 \\
\hline $\mathrm{H}$ & 1.60917700 & -4.70249300 & -1.17883900 \\
\hline $\mathrm{C}$ & 1.81215800 & -2.38392000 & 3.55433400 \\
\hline $\mathrm{C}$ & 2.82329400 & -3.90384000 & 1.93719200 \\
\hline $\mathrm{O}$ & 3.73538800 & -5.35303600 & 0.34631500 \\
\hline $\mathrm{C}$ & 2.85094000 & -2.64515200 & 4.42198900 \\
\hline $\mathrm{H}$ & 1.01397200 & -1.69298300 & 3.80915500 \\
\hline $\mathrm{C}$ & 3.86923300 & -4.16312700 & 2.84412900 \\
\hline $\mathrm{C}$ & 3.88076800 & -3.53993700 & 4.07276900 \\
\hline
\end{tabular}




\begin{tabular}{|c|c|c|c|}
\hline $\mathrm{H}$ & 2.87221500 & -2.15414000 & 5.38931700 \\
\hline $\mathrm{H}$ & 4.65309600 & -4.85479000 & 2.55735400 \\
\hline $\mathrm{H}$ & 4.68386100 & -3.73725800 & 4.77415000 \\
\hline $\mathrm{N}$ & 0.77290100 & -2.75455500 & 1.41730400 \\
\hline $\mathrm{C}$ & 2.36392000 & -0.69870600 & -2.41450000 \\
\hline $\mathrm{C}$ & 3.41179900 & -1.31047900 & -3.14570600 \\
\hline $\mathrm{C}$ & 3.80989100 & 1.17957500 & -1.94881500 \\
\hline $\mathrm{C}$ & 4.61495300 & -0.65405000 & -3.26149000 \\
\hline $\mathrm{H}$ & 3.24721900 & -2.26585500 & -3.62555500 \\
\hline $\mathrm{C}$ & 4.83081600 & 0.60323900 & -2.66960800 \\
\hline $\mathrm{H}$ & 5.41183100 & -1.11265700 & -3.83871300 \\
\hline $\mathrm{H}$ & 5.76893400 & 1.13187200 & -2.78145100 \\
\hline $\mathrm{N}$ & 2.62650600 & 0.50630800 & -1.84375600 \\
\hline $\mathrm{C}$ & 3.88003900 & 2.49000300 & -1.26183000 \\
\hline $\mathrm{C}$ & 2.95008500 & 3.49517300 & -1.60971200 \\
\hline $\mathrm{C}$ & 4.84845600 & 2.70106400 & -0.25501300 \\
\hline $\mathrm{C}$ & 2.03208100 & 3.37603600 & -2.70287400 \\
\hline $\mathrm{C}$ & 2.94663000 & 4.72373500 & -0.86813400 \\
\hline $\mathrm{C}$ & 4.84489300 & 3.95112900 & 0.45185400 \\
\hline $\mathrm{C}$ & 5.81756600 & 1.72190900 & 0.14175400 \\
\hline $\mathrm{C}$ & 1.14934600 & 4.37438300 & -2.99141900 \\
\hline $\mathrm{H}$ & 2.04700500 & 2.48580000 & -3.32312100 \\
\hline $\mathrm{C}$ & 1.99344000 & 5.73970300 & -1.19189900 \\
\hline $\mathrm{C}$ & 3.88704600 & 4.91597300 & 0.14234400 \\
\hline $\mathrm{C}$ & 5.81372100 & 4.18605700 & 1.47801500 \\
\hline $\mathrm{H}$ & 5.82836300 & 0.75208400 & -0.34341500 \\
\hline $\mathrm{C}$ & 6.72571000 & 1.98359400 & 1.12709100 \\
\hline $\mathrm{C}$ & 1.11342800 & 5.57002200 & -2.21710000 \\
\hline
\end{tabular}




\begin{tabular}{|c|c|c|c|}
\hline $\mathrm{H}$ & 0.46749600 & 4.26277700 & -3.82827100 \\
\hline $\mathrm{H}$ & 2.00136600 & 6.65547200 & -0.60755900 \\
\hline F & 3.88248800 & 5.85176600 & 0.69701100 \\
\hline $\mathrm{C}$ & 6.73553900 & 3.23770000 & 1.80448400 \\
\hline F & 5.79524400 & 5.14478000 & 1.98879000 \\
\hline F & 7.45099100 & 1.22460000 & 1.40313600 \\
\hline $\mathrm{H}$ & 0.39799700 & 6.34738300 & -2.46376200 \\
\hline $\mathrm{H}$ & 7.47151700 & 3.42523700 & 2.57906400 \\
\hline C & -7.38674300 & 0.52812700 & -1.07810600 \\
\hline C & -7.02244600 & 1.78173700 & -0.60878400 \\
\hline $\mathrm{C}$ & -5.67775200 & 2.13857400 & -0.45679700 \\
\hline C & -4.73290300 & 1.16782100 & -0.78368000 \\
\hline $\mathrm{C}$ & -5.05644900 & -0.09522300 & -1.29337300 \\
\hline $\mathrm{C}$ & -6.40898500 & -0.39878900 & -1.43218600 \\
\hline $\mathrm{H}$ & -8.43644100 & 0.27263700 & -1.18192100 \\
\hline $\mathrm{H}$ & -7.79225600 & 2.50512700 & -0.35434000 \\
\hline C & -5.30976900 & 3.53548800 & 0.00997700 \\
\hline C & -3.94910400 & -1.04042200 & -1.72504700 \\
\hline $\mathrm{H}$ & -6.70676300 & -1.37042200 & -1.81210500 \\
\hline $\mathrm{C}$ & -5.81959500 & 4.58648100 & -0.98464100 \\
\hline $\mathrm{H}$ & -4.21828700 & 3.61635400 & 0.03846500 \\
\hline $\mathrm{C}$ & -5.84435300 & 3.81036000 & 1.41955300 \\
\hline $\mathrm{H}$ & -5.52851900 & 4.80211700 & 1.75889900 \\
\hline $\mathrm{H}$ & -5.47745700 & 3.06294700 & 2.12674900 \\
\hline $\mathrm{H}$ & -6.93941700 & 3.78984400 & 1.42951600 \\
\hline $\mathrm{H}$ & -5.50997400 & 5.58807000 & -0.66982300 \\
\hline $\mathrm{H}$ & -6.91310300 & 4.57821700 & -1.03912400 \\
\hline $\mathrm{H}$ & -5.43135300 & 4.40317300 & -1.99070000 \\
\hline
\end{tabular}




$\begin{array}{llll}\mathrm{H} & -3.16427100 & -1.00894700 & -0.95901200 \\ \mathrm{C} & -3.35568700 & -0.52822400 & -3.04713100 \\ \mathrm{C} & -4.40714000 & -2.49050300 & -1.86915000 \\ \mathrm{H} & -2.59674300 & -1.21984800 & -3.43347700 \\ \mathrm{H} & -2.89051500 & 0.45191800 & -2.91607200 \\ \mathrm{H} & -4.14138600 & -0.44645500 & -3.80606500 \\ \mathrm{H} & -5.10422200 & -2.61420800 & -2.70542300 \\ \mathrm{H} & -4.88808500 & -2.85914400 & -0.95837300 \\ \mathrm{H} & -3.54243500 & -3.13365400 & -2.06995400 \\ \mathrm{C} & 3.72754500 & -6.00733100 & -0.91349500 \\ \mathrm{H} & 4.61933800 & -6.63062200 & -0.93125700 \\ \mathrm{H} & 3.76907400 & -5.27469000 & -1.72730500 \\ \mathrm{H} & 2.83528000 & -6.63265100 & -1.01831200 \\ \mathrm{H} & -1.32372400 & -2.31505900 & -2.20481400 \\ \mathrm{H} & 1.84548300 & 0.96614100 & -1.33137700 \\ \mathrm{H} & -4.85814500 & -1.79525800 & 1.09420700 \\ \mathrm{H} & 0.09196100 & -1.94115200 & 1.62104900\end{array}$

\section{Structure anti-(2S,3S)-TS}

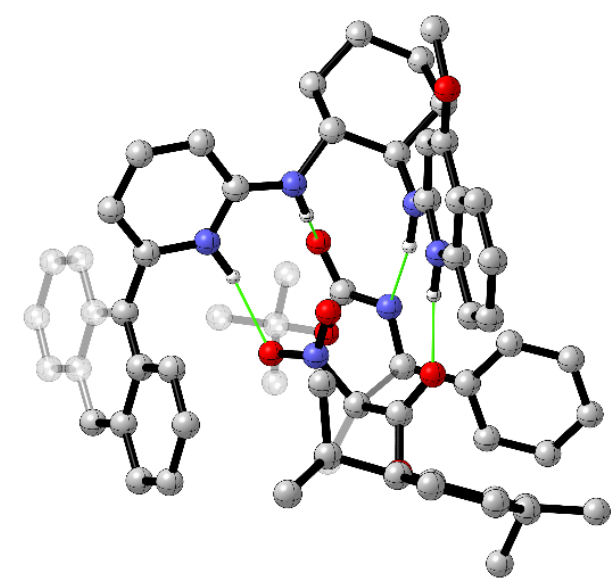

- Thermochemistry - 
Zero-point correction $=$

Thermal correction to Energy=

Thermal correction to Enthalpy=

Thermal correction to Gibbs Free Energy=

Sum of electronic and zero-point Energies=

Sum of electronic and thermal Energies=

Sum of electronic and thermal Enthalpies=

Sum of electronic and thermal Free Energies=
1.224929 (Hartree/Particle)

1.292780

1.293724

1.122464

$-3256.494681$

$-3256.426831$

$-3256.425887$

$-3256.597146$

Number of Imaginary Frequencies $=1$

E (Single Point Energy) $\left[\operatorname{SMD}_{\text {(Toluene) }}\right.$ M06-2X/6-311++G(2d,2p) $]=-3258.788448$

$\begin{array}{lrrr}\mathrm{O} & -2.49844900 & -0.65991600 & 0.36092100 \\ \mathrm{C} & -1.85914700 & -1.68989800 & 0.32270300 \\ \mathrm{O} & -2.41174100 & -2.84664700 & -0.08238000 \\ \mathrm{C} & -0.43916000 & -1.84758600 & 0.73590000 \\ \mathrm{C} & 0.10360000 & -3.25391100 & 0.80287400 \\ \mathrm{H} & 1.09895000 & -3.24745100 & 1.24880000 \\ \mathrm{H} & 0.16673900 & -3.71555500 & -0.18832900 \\ \mathrm{H} & -0.56520800 & -3.85878500 & 1.42116400 \\ \mathrm{~N} & 0.41632300 & -0.92850200 & 0.06632000 \\ \mathrm{O} & -0.00469600 & 0.14376400 & -0.36250900 \\ \mathrm{O} & 1.63185500 & -1.16991700 & 0.10208100 \\ \mathrm{C} & -0.20665600 & -1.05632400 & 2.55971900 \\ \mathrm{C} & -1.54568400 & -1.13211800 & 3.20743900 \\ \mathrm{C} & -1.90919200 & -2.31805700 & 3.85318600 \\ \mathrm{C} & -2.39895500 & -0.02713100 & 3.28293900 \\ \mathrm{C} & -3.09246600 & -2.39323700 & 4.58406900 \\ \mathrm{C} & -3.59194000 & -0.10888400 & 3.99041500 \\ \mathrm{C} & -3.93596500 & -1.28785800 & 4.65228700 \\ \mathrm{C} & 1.73217000 & 0.19564400 & 2.51604200 \\ \mathrm{O} & 2.39795300 & 1.19036700 & 2.17897700 \\ \mathrm{O} & 2.32689800 & -0.89127300 & 3.03314600 \\ \mathrm{C} & 3.77638000 & -1.04212400 & 3.06597300 \\ \mathrm{C} & 4.39664500 & 0.00132400 & 3.99003800 \\ \mathrm{H} & 4.29923500 & 1.00392500 & 3.57396300 \\ \mathrm{H} & 5.45801600 & -0.22603800 & 4.13011800\end{array}$




\begin{tabular}{|c|c|c|c|}
\hline $\mathrm{H}$ & 3.90804100 & -0.02938900 & 4.96865900 \\
\hline $\mathrm{C}$ & 4.35606900 & -0.99128600 & 1.65452500 \\
\hline $\mathrm{H}$ & 3.84153100 & -1.70818000 & 1.00584000 \\
\hline $\mathrm{H}$ & 5.41868000 & -1.25812200 & 1.68485400 \\
\hline $\mathrm{H}$ & 4.26951600 & 0.01043700 & 1.22719100 \\
\hline $\mathrm{C}$ & 3.94682300 & -2.43953100 & 3.65319600 \\
\hline $\mathrm{H}$ & 3.47385900 & -3.18497800 & 3.00645600 \\
\hline $\mathrm{H}$ & 3.48856300 & -2.49477600 & 4.64456100 \\
\hline $\mathrm{H}$ & 5.00928200 & -2.68133500 & 3.74418600 \\
\hline $\mathrm{N}$ & 0.37586800 & 0.16401600 & 2.44603700 \\
\hline $\mathrm{H}$ & 0.43697600 & -1.88672000 & 2.85367900 \\
\hline $\mathrm{H}$ & -4.85612800 & -1.33953600 & 5.22617800 \\
\hline $\mathrm{H}$ & -3.34817200 & -3.30918400 & 5.10837200 \\
\hline $\mathrm{H}$ & -4.24665200 & 0.75496500 & 4.04772300 \\
\hline $\mathrm{H}$ & -2.10394700 & 0.90492800 & 2.81079600 \\
\hline $\mathrm{H}$ & -1.23876400 & -3.17404600 & 3.81858500 \\
\hline $\mathrm{C}$ & 1.07246600 & 5.64811600 & 2.89850300 \\
\hline $\mathrm{C}$ & 2.06584700 & 5.98599800 & 1.78475300 \\
\hline $\mathrm{C}$ & 2.55467300 & 4.71318300 & 1.09117800 \\
\hline $\mathrm{C}$ & 1.38487100 & 3.91558300 & 0.49866400 \\
\hline $\mathrm{C}$ & 0.34191900 & 3.62522900 & 1.60383700 \\
\hline $\mathrm{C}$ & -0.13166500 & 4.88284600 & 2.34183600 \\
\hline $\mathrm{H}$ & 3.06312000 & 4.06292800 & 1.81514100 \\
\hline $\mathrm{H}$ & 2.91928800 & 6.53910600 & 2.18751500 \\
\hline $\mathrm{H}$ & 1.58147700 & 6.64520100 & 1.04966800 \\
\hline $\mathrm{H}$ & 0.73074600 & 6.55889400 & 3.39893700 \\
\hline $\mathrm{H}$ & 1.57522500 & 5.03564000 & 3.65772500 \\
\hline $\mathrm{H}$ & 0.92594600 & 4.47625100 & -0.32766800 \\
\hline $\mathrm{H}$ & 0.87135600 & 3.01136300 & 2.34364200 \\
\hline $\mathrm{H}$ & -0.80761700 & 4.57366700 & 3.14496400 \\
\hline $\mathrm{H}$ & -0.70255500 & 5.55399600 & 1.69132400 \\
\hline $\mathrm{H}$ & 3.28035000 & 4.94565000 & 0.30332100 \\
\hline $\mathrm{N}$ & 1.80001300 & 2.60836200 & 0.01529300 \\
\hline $\mathrm{H}$ & 1.84909800 & 1.90642200 & 0.78145300 \\
\hline $\mathrm{N}$ & -0.71988500 & 2.73151400 & 1.13775300 \\
\hline $\mathrm{H}$ & -0.56568700 & 1.74776300 & 1.41536700 \\
\hline $\mathrm{C}$ & -1.63032400 & 2.99768200 & 0.19460400 \\
\hline $\mathrm{C}$ & -1.80464500 & 4.27940300 & -0.40443500 \\
\hline $\mathrm{C}$ & -3.49520500 & 2.15830100 & -1.08305600 \\
\hline $\mathrm{H}$ & -2.27037200 & 1.04957500 & 0.16358500 \\
\hline $\mathrm{C}$ & -2.81019200 & 4.47886900 & -1.31622700 \\
\hline $\mathrm{H}$ & -1.14186300 & 5.07947300 & -0.12572400 \\
\hline $\mathrm{C}$ & -4.34839100 & 1.08137500 & -1.38187600 \\
\hline $\mathrm{C}$ & -3.70703300 & 3.41255000 & -1.67974800 \\
\hline $\mathrm{O}$ & -3.04735400 & 5.64032500 & -1.92372200 \\
\hline $\mathrm{C}$ & -5.38202800 & 1.27707100 & -2.27578900 \\
\hline
\end{tabular}




\begin{tabular}{|c|c|c|c|}
\hline $\mathrm{H}$ & -4.19075500 & 0.11646500 & -0.91006800 \\
\hline $\mathrm{C}$ & -4.76789700 & 3.58623100 & -2.58827800 \\
\hline $\mathrm{C}$ & -5.59639200 & 2.52615700 & -2.88525900 \\
\hline $\mathrm{H}$ & -6.03740600 & 0.44166500 & -2.50203600 \\
\hline $\mathrm{H}$ & -4.91658800 & 4.56090200 & -3.03841400 \\
\hline $\mathrm{H}$ & -6.41643600 & 2.65573100 & -3.58284400 \\
\hline $\mathrm{N}$ & -2.44712400 & 2.00046800 & -0.19043900 \\
\hline $\mathrm{C}$ & 2.53450200 & 2.33715700 & -1.05468100 \\
\hline $\mathrm{C}$ & 2.92960800 & 3.24568700 & -2.06941400 \\
\hline $\mathrm{C}$ & 3.78418100 & 0.59393100 & -2.15938700 \\
\hline $\mathrm{H}$ & 2.56811000 & 0.34294200 & -0.52646300 \\
\hline $\mathrm{C}$ & 3.74068300 & 2.79983400 & -3.08394300 \\
\hline $\mathrm{H}$ & 2.60280800 & 4.27655600 & -2.01365900 \\
\hline $\mathrm{C}$ & 4.19833900 & 1.46505600 & -3.13480400 \\
\hline $\mathrm{H}$ & 4.05061600 & 3.49621500 & -3.85697400 \\
\hline $\mathrm{H}$ & 4.86385900 & 1.11809800 & -3.91472100 \\
\hline $\mathrm{N}$ & 2.95083800 & 1.04686300 & -1.18133300 \\
\hline $\mathrm{C}$ & 4.19310000 & -0.82852400 & -2.02428300 \\
\hline $\mathrm{C}$ & 5.45883800 & -1.12194700 & -1.48221000 \\
\hline $\mathrm{C}$ & 3.27387300 & -1.84226500 & -2.34226100 \\
\hline $\mathrm{C}$ & 6.40504200 & -0.11336400 & -1.10946300 \\
\hline $\mathrm{C}$ & 5.80593600 & -2.49540600 & -1.24743300 \\
\hline $\mathrm{C}$ & 3.63271800 & -3.20868600 & -2.09282800 \\
\hline $\mathrm{C}$ & 1.98620600 & -1.57033600 & -2.90574500 \\
\hline $\mathrm{C}$ & 7.60630500 & -0.45079500 & -0.55826600 \\
\hline $\mathrm{H}$ & 6.15701300 & 0.93176800 & -1.26514800 \\
\hline $\mathrm{C}$ & 7.07876200 & -2.80482500 & -0.67302900 \\
\hline $\mathrm{C}$ & 4.88651000 & -3.49892400 & -1.55469800 \\
\hline $\mathrm{C}$ & 2.69378000 & -4.24436900 & -2.39903000 \\
\hline $\mathrm{H}$ & 1.69598200 & -0.54258900 & -3.10760700 \\
\hline $\mathrm{C}$ & 1.12763400 & -2.58807200 & -3.19659100 \\
\hline $\mathrm{C}$ & 7.95391600 & -1.81608300 & -0.33710600 \\
\hline $\mathrm{H}$ & 8.30993200 & 0.32770500 & -0.28112400 \\
\hline $\mathrm{H}$ & 7.32756500 & -3.84938100 & -0.50779800 \\
\hline $\mathrm{H}$ & 5.15314900 & -4.53614700 & -1.36363100 \\
\hline $\mathrm{C}$ & 1.47742500 & -3.94667600 & -2.93564400 \\
\hline $\mathrm{H}$ & 2.98053600 & -5.27357900 & -2.20188000 \\
\hline $\mathrm{H}$ & 0.16765400 & -2.36739800 & -3.64873600 \\
\hline $\mathrm{H}$ & 8.91684600 & -2.05905500 & 0.09961200 \\
\hline $\mathrm{H}$ & 0.77165000 & -4.73548000 & -3.17783900 \\
\hline $\mathrm{C}$ & -6.13625000 & -2.51054500 & -1.88505200 \\
\hline $\mathrm{C}$ & -4.98210500 & -2.40374700 & -2.64980400 \\
\hline $\mathrm{C}$ & -3.71806000 & -2.51744400 & -2.06127700 \\
\hline $\mathrm{C}$ & -3.68173800 & -2.73558200 & -0.68470500 \\
\hline $\mathrm{C}$ & -4.81643000 & -2.85287000 & 0.12333400 \\
\hline $\mathrm{C}$ & -6.05247900 & -2.73601800 & -0.51293000 \\
\hline
\end{tabular}




\begin{tabular}{lrrr}
$\mathrm{H}$ & -7.10964600 & -2.42685600 & -2.35887000 \\
$\mathrm{H}$ & -5.05925900 & -2.23312800 & -3.71964600 \\
$\mathrm{C}$ & -2.45110600 & -2.37355300 & -2.88681800 \\
$\mathrm{C}$ & -4.66819300 & -3.10648100 & 1.61188800 \\
$\mathrm{H}$ & -6.96392600 & -2.81573600 & 0.06886700 \\
$\mathrm{C}$ & -2.54477600 & -3.13890900 & -4.21140400 \\
$\mathrm{H}$ & -1.62631500 & -2.80626100 & -2.30718900 \\
$\mathrm{C}$ & -2.13889900 & -0.88956600 & -3.13523700 \\
$\mathrm{H}$ & -2.99040200 & -0.39938000 & -3.62064600 \\
$\mathrm{H}$ & -1.26986100 & -0.77566400 & -3.79295300 \\
$\mathrm{H}$ & -1.92815500 & -0.35819200 & -2.20126100 \\
$\mathrm{H}$ & -1.57849200 & -3.13550900 & -4.72682700 \\
$\mathrm{H}$ & -3.26812800 & -2.67687800 & -4.89068300 \\
$\mathrm{H}$ & -2.84393000 & -4.17834500 & -4.05085300 \\
$\mathrm{H}$ & -3.93515500 & -2.38239700 & 1.99254200 \\
$\mathrm{C}$ & -4.12693000 & -4.52099000 & 1.86632800 \\
$\mathrm{C}$ & -5.96661400 & -2.89984900 & 2.38865500 \\
$\mathrm{H}$ & -3.97589900 & -4.67844100 & 2.93916000 \\
$\mathrm{H}$ & -3.17499100 & -4.68994400 & 1.35597900 \\
$\mathrm{H}$ & -4.84317500 & -5.26919700 & 1.51013600 \\
$\mathrm{H}$ & -6.72872400 & -3.62919100 & 2.09285600 \\
$\mathrm{H}$ & -6.37224500 & -1.89346900 & 2.24405300 \\
$\mathrm{H}$ & -5.77863600 & -3.03968500 & 3.45648400 \\
$\mathrm{C}$ & -2.23549500 & 6.75914700 & -1.59712500 \\
$\mathrm{H}$ & -1.18941000 & 6.56535700 & -1.85696600 \\
$\mathrm{H}$ & -2.31983000 & 6.99784400 & -0.53185900 \\
$\mathrm{H}$ & -2.61619200 & 7.58627700 & -2.19285600 \\
$\mathrm{St}$ & $-161-(2 R, 3 R)$ & -152 & \\
\hline
\end{tabular}

\section{Structure anti-(2R,3R)-TS2}

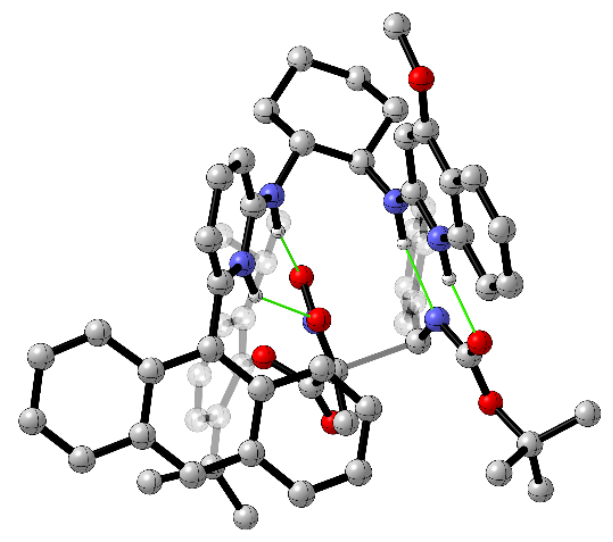

- Thermochemistry -

Zero-point correction=

1.224932 (Hartree/Particle) 
Thermal correction to Energy $=\quad 1.292984$

Thermal correction to Enthalpy= $\quad 1.293928$

Thermal correction to Gibbs Free Energy= $\quad 1.120112$

Sum of electronic and zero-point Energies $=\quad-3256.479046$

Sum of electronic and thermal Energies $=\quad-3256.410994$

Sum of electronic and thermal Enthalpies $=\quad-3256.410050$

Sum of electronic and thermal Free Energies $=\quad-3256.583866$

Number of Imaginary Frequencies $=1$

E (Single Point Energy) $\left[\operatorname{SMD}_{\text {(Toluene) }}\right.$ M06-2X/6-311++G(2d,2p) $]=-3258.778203$

$\begin{array}{lrrr}\mathrm{O} & -4.14964000 & 0.59401200 & 1.83958500 \\ \mathrm{C} & -3.39428100 & 0.54867000 & 0.89817000 \\ \mathrm{O} & -3.75277400 & 0.72185600 & -0.38251400 \\ \mathrm{C} & -1.92938400 & 0.37629700 & 1.11405300 \\ \mathrm{C} & -1.33460200 & 1.31749700 & 2.12966400 \\ \mathrm{H} & -1.16296100 & 2.31003900 & 1.69660100 \\ \mathrm{H} & -0.36704600 & 0.95027000 & 2.47797200 \\ \mathrm{H} & -2.02710200 & 1.40801100 & 2.96850900 \\ \mathrm{~N} & -1.11232300 & 0.11716000 & 0.02067700 \\ \mathrm{O} & 0.09378800 & 0.44433300 & 0.10890500 \\ \mathrm{O} & -1.50152000 & -0.57463700 & -0.93865300 \\ \mathrm{C} & -1.71205500 & -1.47307100 & 2.02342200 \\ \mathrm{C} & -2.57992700 & -2.42541100 & 1.28321900 \\ \mathrm{C} & -3.96934100 & -2.40898900 & 1.47157200 \\ \mathrm{C} & -2.01729300 & -3.45135500 & 0.51547800 \\ \mathrm{C} & -4.77409600 & -3.36632200 & 0.86308300 \\ \mathrm{C} & -2.82278800 & -4.40350200 & -0.09887200 \\ \mathrm{C} & -4.20596100 & -4.35852500 & 0.06722400 \\ \mathrm{C} & 0.32341900 & -1.33446100 & 3.12134200 \\ \mathrm{O} & 1.56426500 & -1.26839600 & 3.12236800 \\ \mathrm{O} & -0.39554900 & -1.10983500 & 4.22788900 \\ \mathrm{C} & 0.21124600 & -0.81133600 & 5.51894500 \\ \mathrm{C} & 1.01619300 & 0.48269800 & 5.44529300 \\ \mathrm{H} & 1.95797700 & 0.33891900 & 4.91293400 \\ \mathrm{H} & 1.23668500 & 0.83047100 & 6.45925900 \\ \mathrm{H} & 0.42733300 & 1.25524500 & 4.93854200 \\ \mathrm{C} & 1.05108100 & -1.99450400 & 5.98754000\end{array}$




$\begin{array}{lrrr}\mathrm{H} & 0.44373100 & -2.90429000 & 5.99930700 \\ \mathrm{H} & 1.40448300 & -1.80579600 & 7.00580300 \\ \mathrm{H} & 1.91408500 & -2.14904700 & 5.33842300 \\ \mathrm{C} & -1.00660100 & -0.62439200 & 6.41732600 \\ \mathrm{H} & -1.61989700 & -1.52934400 & 6.41812200 \\ \mathrm{H} & -1.61820500 & 0.21072000 & 6.06284500 \\ \mathrm{H} & -0.68832100 & -0.41548800 & 7.44216900 \\ \mathrm{~N} & -0.38384600 & -1.69469500 & 2.02302400 \\ \mathrm{H} & -2.18143800 & -1.05206000 & 2.91515500 \\ \mathrm{H} & -4.83632200 & -5.10740900 & -0.40248400 \\ \mathrm{H} & -5.84794500 & -3.34063000 & 1.01966900 \\ \mathrm{H} & -2.37068900 & -5.20677900 & -0.67477600 \\ \mathrm{H} & -0.93751000 & -3.53876900 & 0.47306000 \\ \mathrm{H} & -4.41877000 & -1.65284800 & 2.10550200 \\ \mathrm{C} & -0.40325000 & -4.71008800 & -3.16848900 \\ \mathrm{C} & 0.01301200 & -3.90827500 & -4.40363100 \\ \mathrm{C} & 0.02072000 & -2.40640600 & -4.10947600 \\ \mathrm{C} & 0.93832600 & -2.08400800 & -2.92775200 \\ \mathrm{C} & 0.50754700 & -2.90394500 & -1.68885400 \\ \mathrm{C} & 0.51468500 & -4.40897100 & -1.97981300 \\ \mathrm{H} & -0.99147800 & -2.06294000 & -3.86125400 \\ \mathrm{H} & -0.65976700 & -4.11724100 & -5.24018400 \\ \mathrm{H} & 1.01719100 & -4.22406200 & -4.72106800 \\ \mathrm{H} & -0.39359000 & -5.78229400 & -3.38570500 \\ \mathrm{C} & -1.43477700 & -4.44561200 & -2.89759400 \\ \mathrm{H} & 1.96578600 & -2.33456200 & -3.20561900 \\ \mathrm{H} & -0.53470700 & -2.62199300 & -1.50197300 \\ \mathrm{H} & 0.18282900 & -4.94106900 & -1.08152300 \\ \mathrm{H} & 1.52986200 & -4.76576600 & -2.19836900 \\ \mathrm{H} & 0.34820600 & -1.83201400 & -4.98262600 \\ \mathrm{H} & 0.89397500 & -0.67220300 & -2.57385500 \\ \mathrm{~N} & 0.10501700 & -0.43262400 & -1.96258900 \\ \mathrm{H} & 1.19339800 & -2.51080100 & -0.45631500 \\ \mathrm{~N} & 0.60210700 & -2.12546800 & 0.30209800 \\ \mathrm{H} & 2.51247700 & -2.45718100 & -0.24584400 \\ \mathrm{C} & 3.48711700 & -2.94132000 & -1.16914400 \\ \mathrm{C} & 5.27153000 & -1.78041400 & 1.24220600 \\ \mathrm{C} & 5.83535000 & -1.63088200 & 1.65524100 \\ \mathrm{H} & & & \end{array}$




\begin{tabular}{|c|c|c|c|}
\hline & 6.97130300 & -1.47950300 & 1.88253100 \\
\hline & 6.25212500 & -0.65012800 & 3.74195500 \\
\hline & 7.37724600 & -2.36808900 & -0.03282500 \\
\hline & 8.01620900 & -1.35634400 & 2.14450100 \\
\hline $\mathbf{N}$ & 2.93662600 & -1.91403000 & 0.90968300 \\
\hline & 1.92186000 & 0.17546700 & -2.63564300 \\
\hline $\mathrm{C}$ & 3.08125300 & 0.00532200 & -3.43184900 \\
\hline & 2.77517700 & 2.31290800 & -1.89181400 \\
\hline $\mathrm{H}$ & 1.04946100 & 1.38571000 & -1.24566900 \\
\hline $\mathrm{C}$ & 4.04509500 & 0.98426600 & -3.42781600 \\
\hline $\mathrm{H}$ & 3.17153400 & -0.86904500 & -4.06261000 \\
\hline $\mathrm{C}$ & 3.90492400 & 2.15414200 & -2.65803500 \\
\hline $\mathrm{H}$ & 4.93072600 & 0.85627000 & -4.04240900 \\
\hline $\mathrm{H}$ & 4.66181400 & 2.92806700 & -2.65298100 \\
\hline $\mathrm{N}$ & 1.83609800 & 1.32011700 & -1.90515100 \\
\hline $\mathrm{C}$ & 2.50072900 & 3.48957600 & -1.03646700 \\
\hline $\mathrm{C}$ & 2.39710000 & 4.76409300 & -1.63550800 \\
\hline $\mathrm{C}$ & 2.35879300 & 3.31349700 & 0.35783800 \\
\hline $\mathrm{C}$ & 2.48003200 & 4.98738300 & -3.04843300 \\
\hline $\mathrm{C}$ & 2.15771000 & 5.90325100 & -0.79421000 \\
\hline $\mathrm{C}$ & 2.09922000 & 4.46553200 & 1.17510300 \\
\hline $\mathrm{C}$ & 2.48656200 & 2.04700800 & 1.01439700 \\
\hline $\mathrm{C}$ & 2.37264800 & 6.24407700 & -3.56906300 \\
\hline $\mathrm{H}$ & 2.61555300 & 4.14421000 & -3.71659800 \\
\hline $\mathrm{C}$ & 2.05733400 & 7.20393000 & -1.38060200 \\
\hline $\mathrm{C}$ & 2.01328400 & 5.72389500 & 0.58074600 \\
\hline $\mathrm{C}$ & 1.94914300 & 4.30689300 & 2.58810200 \\
\hline $\mathrm{H}$ & 2.70812000 & 1.15439400 & 0.43759200 \\
\hline $\mathrm{C}$ & 2.32137200 & 1.93353300 & 2.36381800 \\
\hline $\mathrm{C}$ & 2.16808300 & 7.37511400 & -2.72681000 \\
\hline $\mathrm{H}$ & 2.43518800 & 6.38660500 & -4.64318800 \\
\hline $\mathrm{H}$ & 1.88507400 & 8.05074700 & -0.72235300 \\
\hline $\mathrm{H}$ & 1.82662300 & 6.59214000 & 1.20901000 \\
\hline $\mathrm{C}$ & 2.05020100 & 3.07845700 & 3.16788500 \\
\hline $\mathrm{H}$ & 1.75730600 & 5.19349000 & 3.18589100 \\
\hline $\mathrm{H}$ & 2.38117500 & 0.95161000 & 2.82429100 \\
\hline $\mathrm{H}$ & 2.09007800 & 8.36400900 & -3.16558300 \\
\hline $\mathrm{H}$ & 1.93897300 & 2.96646700 & 4.24154100 \\
\hline $\mathrm{C}$ & -7.77415500 & 0.85942800 & -1.39865400 \\
\hline $\mathrm{C}$ & -7.22988600 & 1.89317100 & -0.65094300 \\
\hline $\mathrm{C}$ & -5.88000700 & 1.89108800 & -0.28367600 \\
\hline $\mathrm{C}$ & -5.12304000 & 0.79322700 & -0.68909200 \\
\hline $\mathrm{C}$ & -5.62091500 & -0.25205700 & -1.47729900 \\
\hline $\mathrm{C}$ & -6.97124900 & -0.19761700 & -1.81986400 \\
\hline $\mathrm{H}$ & -8.82505700 & 0.88105400 & -1.66911600 \\
\hline $\mathrm{H}$ & -7.85869800 & 2.72496300 & -0.34529200 \\
\hline
\end{tabular}




$\begin{array}{lrrr}\mathrm{C} & -5.29926300 & 3.07025900 & 0.47664200 \\ \mathrm{C} & -4.68675600 & -1.33314700 & -1.99446000 \\ \mathrm{H} & -7.40460900 & -0.98778500 & -2.42370400 \\ \mathrm{C} & -5.44213400 & 4.36095000 & -0.34033300 \\ \mathrm{H} & -4.22876000 & 2.89627800 & 0.62449300 \\ \mathrm{C} & -5.95078600 & 3.21922500 & 1.85550500 \\ \mathrm{H} & -5.49208800 & 4.04785300 & 2.40486700 \\ \mathrm{H} & -5.83608400 & 2.30294300 & 2.43824700 \\ \mathrm{H} & -7.01961700 & 3.43781900 & 1.75677700 \\ \mathrm{H} & -4.97479000 & 5.19773900 & 0.18864200 \\ \mathrm{H} & -6.49583900 & 4.61393000 & -0.49651500 \\ \mathrm{H} & -4.97050400 & 4.26422400 & -1.32260800 \\ \mathrm{H} & -4.01419200 & -1.62552100 & -1.17882500 \\ \mathrm{C} & -3.81484600 & -0.76414900 & -3.12285400 \\ \mathrm{C} & -5.42290100 & -2.57896400 & -2.48562300 \\ \mathrm{H} & -3.14726900 & -1.54537200 & -3.50568300 \\ \mathrm{H} & -3.20363500 & 0.06856400 & -2.76712700 \\ \mathrm{H} & -4.44142300 & -0.41728000 & -3.95197900 \\ \mathrm{H} & -5.98420700 & -2.37445900 & -3.40423600 \\ \mathrm{H} & -6.12168000 & -2.96247800 & -1.73569900 \\ \mathrm{H} & -4.69973200 & -3.36989800 & -2.70974600 \\ \mathrm{C} & 5.45885600 & -3.88168900 & -2.89903300 \\ \mathrm{H} & 4.87392300 & -3.21636600 & -3.54428600 \\ \mathrm{H} & 4.89364800 & -4.79674400 & -2.69558900 \\ \mathrm{H} & 6.40164900 & -4.12938500 & -3.38251000\end{array}$

\section{Structure syn-(2R,3S)-TS2}

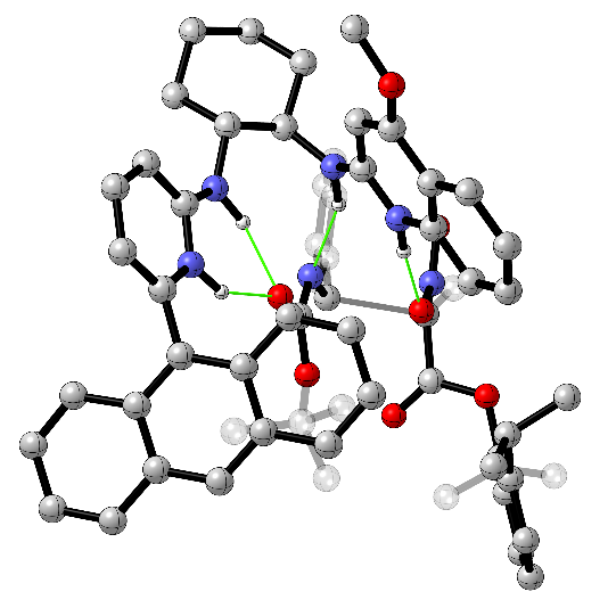

- Thermochemistry -

Zero-point correction=

1.223987 (Hartree/Particle) 


$\begin{array}{ll}\text { Thermal correction to Energy= } & 1.292090 \\ \text { Thermal correction to Enthalpy= } & 1.293034 \\ \text { Thermal correction to Gibbs Free Energy= } & 1.121053 \\ \text { Sum of electronic and zero-point Energies }= & -3256.486098 \\ \text { Sum of electronic and thermal Energies }= & -3256.417995 \\ \text { Sum of electronic and thermal Enthalpies }= & -3256.417051 \\ \text { Sum of electronic and thermal Free Energies }= & -3256.589032\end{array}$

Number of Imaginary Frequencies $=1$

E (Single Point Energy) $\left[\mathrm{SMD}_{\text {(Toluene) }} \mathrm{M} 06-2 \mathrm{X} / 6-311++\mathrm{G}(2 \mathrm{~d}, 2 \mathrm{p})\right]=-3258.785755$

$\begin{array}{llll}\mathrm{O} & 4.06814600 & -0.10187300 & -1.47546100 \\ \mathrm{C} & 3.96473900 & 0.39809400 & -0.38595600 \\ \mathrm{O} & 4.54861900 & -0.08709000 & 0.73401500 \\ \mathrm{C} & 3.30265000 & 1.71637600 & -0.10219500 \\ \mathrm{C} & 4.22815900 & 2.89739400 & -0.04438200 \\ \mathrm{H} & 4.92024900 & 2.79449800 & 0.80054100 \\ \mathrm{H} & 3.66541600 & 3.82468500 & 0.06875100 \\ \mathrm{H} & 4.81365400 & 2.95003500 & -0.96673900 \\ \mathrm{~N} & 2.27596200 & 1.66760200 & 0.79672300 \\ \mathrm{O} & 1.78151000 & 2.70380100 & 1.26612800 \\ \mathrm{O} & 1.68870400 & 0.55451500 & 0.97369300 \\ \mathrm{C} & 1.82577600 & 1.95195900 & -1.77935000 \\ \mathrm{C} & 2.15968900 & 3.34696800 & -2.11275000 \\ \mathrm{C} & 1.53591200 & 4.42621400 & -1.47379000 \\ \mathrm{C} & 3.13970000 & 3.59468300 & -3.07829800 \\ \mathrm{C} & 1.88218000 & 5.72952900 & -1.80856800\end{array}$




\begin{tabular}{|c|c|c|c|}
\hline $\mathrm{C}$ & 3.47621200 & 4.90065700 & -3.42121500 \\
\hline $\mathrm{C}$ & 2.84691300 & 5.96950100 & -2.78769600 \\
\hline $\mathrm{C}$ & 0.18923100 & 0.39077500 & -1.25192200 \\
\hline $\mathrm{O}$ & -0.97245200 & 0.13245400 & -0.87972600 \\
\hline $\mathrm{O}$ & 1.04817000 & -0.53893800 & -1.63172900 \\
\hline $\mathrm{C}$ & 0.76412800 & -1.96877200 & -1.49321400 \\
\hline $\mathrm{C}$ & 0.33569100 & -2.30269300 & -0.06844000 \\
\hline $\mathrm{H}$ & -0.67889200 & -1.97260300 & 0.15598200 \\
\hline $\mathrm{H}$ & 0.38787100 & -3.38733900 & 0.06705700 \\
\hline $\mathrm{C}$ & 2.11206500 & -2.61303700 & -1.79543500 \\
\hline $\mathrm{H}$ & 2.84921200 & -2.34986900 & -1.03053800 \\
\hline $\mathrm{H}$ & 2.49367100 & -2.27234500 & -2.76158500 \\
\hline $\mathrm{H}$ & 2.00060800 & -3.70117400 & -1.82007500 \\
\hline $\mathrm{C}$ & -0.27740800 & -2.38010800 & -2.52805000 \\
\hline $\mathrm{H}$ & -0.43579600 & -3.46216500 & -2.47503400 \\
\hline $\mathrm{H}$ & 0.06450600 & -2.12915500 & -3.53645600 \\
\hline $\mathrm{H}$ & -1.23813300 & -1.89151800 & -2.34816200 \\
\hline $\mathrm{N}$ & 0.60367900 & 1.69244100 & -1.31508300 \\
\hline $\mathrm{H}$ & 2.38824500 & 1.17434100 & -2.29805400 \\
\hline $\mathrm{H}$ & 3.11240900 & 6.98868000 & -3.04998200 \\
\hline $\mathrm{H}$ & 1.40726800 & 6.56358900 & -1.30047400 \\
\hline $\mathrm{H}$ & 4.23070300 & 5.08417600 & -4.17925700 \\
\hline $\mathrm{H}$ & 3.63552800 & 2.75461700 & -3.55928600 \\
\hline $\mathrm{H}$ & 0.81926800 & 4.23204100 & -0.68226800 \\
\hline $\mathrm{C}$ & -2.72391000 & 6.13880200 & -0.69903400 \\
\hline $\mathrm{C}$ & -4.03228200 & 5.58555500 & -1.27128200 \\
\hline $\mathrm{C}$ & -3.85144600 & 4.16287300 & -1.81070600 \\
\hline $\mathrm{C}$ & -3.32157200 & 3.24146300 & -0.70513500 \\
\hline
\end{tabular}




\begin{tabular}{|c|c|c|c|}
\hline $\mathrm{C}$ & -1.96742900 & 3.79975900 & -0.21702500 \\
\hline $\mathrm{C}$ & -2.13688100 & 5.20546900 & 0.36499900 \\
\hline $\mathrm{H}$ & -3.13316000 & 4.15521300 & -2.64000000 \\
\hline $\mathrm{H}$ & -4.40515800 & 6.23778400 & -2.06605300 \\
\hline $\mathrm{H}$ & -4.79949400 & 5.57868900 & -0.48430500 \\
\hline $\mathrm{H}$ & -2.88521800 & 7.13434400 & -0.27561900 \\
\hline $\mathrm{H}$ & -1.99319100 & 6.25277500 & -1.51080200 \\
\hline $\mathrm{H}$ & -4.04651300 & 3.23901200 & 0.12352800 \\
\hline $\mathrm{H}$ & -1.16081800 & 5.56281000 & 0.70785600 \\
\hline $\mathrm{H}$ & -2.79453200 & 5.17952900 & 1.24442500 \\
\hline $\mathrm{H}$ & -4.79575400 & 3.77716800 & -2.20952700 \\
\hline $\mathrm{N}$ & -3.10418600 & 1.86627500 & -1.12910800 \\
\hline $\mathrm{H}$ & -2.13854600 & 1.50596400 & -1.08619000 \\
\hline $\mathrm{N}$ & -1.19061800 & 2.87784700 & 0.60232200 \\
\hline $\mathrm{H}$ & -0.33728600 & 2.53997500 & 0.12458800 \\
\hline $\mathrm{C}$ & -1.55848900 & 2.19780300 & 1.69099300 \\
\hline $\mathrm{C}$ & -2.74752800 & 2.44614300 & 2.43804600 \\
\hline $\mathrm{C}$ & -0.97100700 & 0.43100600 & 3.20081300 \\
\hline $\mathrm{H}$ & 0.15784200 & 1.04465500 & 1.58878100 \\
\hline $\mathrm{C}$ & -3.02344800 & 1.68425500 & 3.54757300 \\
\hline $\mathrm{H}$ & -3.38242900 & 3.26688900 & 2.14294000 \\
\hline $\mathrm{C}$ & -0.03878000 & -0.56011800 & 3.55788700 \\
\hline $\mathrm{C}$ & -2.12910400 & 0.63401000 & 3.96884800 \\
\hline $\mathrm{O}$ & -4.09891800 & 1.84146800 & 4.31831900 \\
\hline $\mathrm{C}$ & -0.27424700 & -1.32162200 & 4.68327300 \\
\hline $\mathrm{H}$ & 0.85297600 & -0.68903400 & 2.94973800 \\
\hline $\mathrm{C}$ & -2.34866100 & -0.15836600 & 5.11351100 \\
\hline $\mathrm{C}$ & -1.42956300 & -1.12452300 & 5.46623000 \\
\hline
\end{tabular}




\begin{tabular}{|c|c|c|c|}
\hline $\mathrm{H}$ & 0.44825700 & -2.07974900 & 4.97171800 \\
\hline $\mathrm{H}$ & -3.24002800 & 0.01198800 & 5.70659300 \\
\hline & -1.59185700 & -1.72986000 & 6.35125300 \\
\hline $\mathrm{N}$ & -0.74086000 & 1.21074600 & 2.09247200 \\
\hline $\mathrm{C}$ & -4.05260900 & 0.92703700 & -1.15665500 \\
\hline $\mathrm{C}$ & -5.44865200 & 1.15740900 & -1.11808100 \\
\hline $\mathrm{C}$ & -4.46886600 & -1.44667800 & -1.25822900 \\
\hline $\mathrm{C}$ & -6.30530800 & 0.08310900 & -1.18507200 \\
\hline $\mathrm{H}$ & -5.81982700 & 2.17207400 & -1.05952500 \\
\hline $\mathrm{C}$ & -5.82684000 & -1.23902600 & -1.26239000 \\
\hline $\mathrm{H}$ & -7.37616500 & 0.26042700 & -1.16669700 \\
\hline $\mathrm{H}$ & -6.49597000 & -2.08966500 & -1.28786500 \\
\hline $\mathrm{N}$ & -3.63842700 & -0.36761100 & -1.22450000 \\
\hline $\mathrm{C}$ & -3.80199900 & -2.76927900 & -1.20192600 \\
\hline $\mathrm{C}$ & -3.79459400 & -3.61298400 & -2.32812300 \\
\hline $\mathrm{C}$ & -3.16375900 & -3.12971300 & 0.00135800 \\
\hline $\mathrm{C}$ & -4.41109200 & -3.26454800 & -3.57197000 \\
\hline $\mathrm{C}$ & -3.11014300 & -4.87214300 & -2.23836700 \\
\hline $\mathrm{C}$ & -2.47245900 & -4.38563500 & 0.06732600 \\
\hline $\mathrm{C}$ & -3.19229700 & -2.31257100 & 1.17948300 \\
\hline $\mathrm{C}$ & -4.36582200 & -4.11742300 & -4.63576100 \\
\hline $\mathrm{H}$ & -4.91631300 & -2.30837400 & -3.66559300 \\
\hline $\mathrm{C}$ & -3.08915600 & -5.73629900 & -3.37771200 \\
\hline $\mathrm{C}$ & -2.46490200 & -5.22092500 & -1.05040500 \\
\hline $\mathrm{C}$ & -1.80639100 & -4.75315000 & 1.27960300 \\
\hline $\mathrm{H}$ & -3.74289100 & -1.37632100 & 1.17379400 \\
\hline $\mathrm{C}$ & -2.54843500 & -2.70223000 & 2.31665900 \\
\hline $\mathrm{C}$ & -3.69985600 & -5.37446200 & -4.54039400 \\
\hline
\end{tabular}




\begin{tabular}{|c|c|c|c|}
\hline $\mathrm{H}$ & -4.83915800 & -3.83738400 & -5.57135800 \\
\hline $\mathrm{H}$ & -2.57190600 & -6.68776300 & -3.29222500 \\
\hline $\mathrm{H}$ & -1.94207400 & -6.17344900 & -0.99440900 \\
\hline $\mathrm{C}$ & -1.83124600 & -3.93355300 & 2.36776200 \\
\hline $\mathrm{H}$ & -1.27958800 & -5.70315300 & 1.30844000 \\
\hline $\mathrm{H}$ & -2.59151100 & -2.07625400 & 3.20248200 \\
\hline $\mathrm{H}$ & -3.67972300 & -6.03519400 & -5.40046100 \\
\hline $\mathrm{H}$ & -1.32204500 & -4.21627100 & 3.28371100 \\
\hline C & 6.52310900 & -3.73748000 & 0.61858800 \\
\hline $\mathrm{C}$ & 7.07306900 & -2.64649000 & -0.03984500 \\
\hline $\mathrm{C}$ & 6.44075100 & -1.39881500 & -0.03423500 \\
\hline $\mathrm{C}$ & 5.22943600 & -1.31253800 & 0.65029000 \\
\hline $\mathrm{C}$ & 4.65329200 & -2.38196300 & 1.34590400 \\
\hline $\mathrm{C}$ & 5.32487200 & -3.60272200 & 1.31510700 \\
\hline $\mathrm{H}$ & 7.03303400 & -4.69538700 & 0.59906300 \\
\hline $\mathrm{H}$ & 8.01552500 & -2.75788600 & -0.56917200 \\
\hline $\mathrm{C}$ & 7.08720800 & -0.21129900 & -0.72409000 \\
\hline $\mathrm{C}$ & 3.38234200 & -2.15892300 & 2.14433500 \\
\hline $\mathrm{H}$ & 4.91008600 & -4.45781700 & 1.83944800 \\
\hline $\mathrm{C}$ & 8.46741800 & 0.08251600 & -0.12314800 \\
\hline $\mathrm{H}$ & 6.46317400 & 0.67161800 & -0.55025900 \\
\hline $\mathrm{C}$ & 7.18288600 & -0.43673900 & -2.23701000 \\
\hline $\mathrm{H}$ & 7.61224300 & 0.44370300 & -2.72617200 \\
\hline $\mathrm{H}$ & 6.19611300 & -0.63225200 & -2.66277100 \\
\hline $\mathrm{H}$ & 7.83228500 & -1.29045200 & -2.45967100 \\
\hline $\mathrm{H}$ & 8.90114900 & 0.97326300 & -0.58841700 \\
\hline $\mathrm{H}$ & 9.15719900 & -0.75046400 & -0.29407000 \\
\hline $\mathrm{H}$ & 8.40351700 & 0.25134300 & 0.95553700 \\
\hline
\end{tabular}




\begin{tabular}{|c|c|c|c|}
\hline $\mathrm{H}$ & 2.76412000 & -1.45756900 & 1.57328700 \\
\hline $\mathrm{C}$ & 3.72195100 & -1.47734500 & 3.47798900 \\
\hline $\mathrm{C}$ & 2.58080000 & -3.43959000 & 2.37719200 \\
\hline $\mathrm{H}$ & 2.81652800 & -1.30332000 & 4.07183500 \\
\hline $\mathrm{H}$ & 4.20825300 & -0.51277900 & 3.31002400 \\
\hline $\mathrm{H}$ & 4.39667100 & -2.10752700 & 4.06746200 \\
\hline $\mathrm{H}$ & 3.08055300 & -4.10181600 & 3.09278300 \\
\hline $\mathrm{H}$ & 2.42872800 & -3.99452100 & 1.44543200 \\
\hline $\mathrm{H}$ & 1.59252500 & -3.19580700 & 2.78692100 \\
\hline $\mathrm{C}$ & -5.01928900 & 2.87470900 & 3.99766900 \\
\hline $\mathrm{H}$ & -5.80605500 & 2.81543100 & 4.74694600 \\
\hline $\mathrm{H}$ & -5.44260100 & 2.71535500 & 2.99982100 \\
\hline $\mathrm{H}$ & -4.53171000 & 3.85345900 & 4.04676100 \\
\hline $\mathrm{H}$ & -1.34783100 & 3.89165300 & -1.12034700 \\
\hline $\mathrm{H}$ & -2.61835500 & -0.52998800 & -1.18059000 \\
\hline $\mathrm{H}$ & 1.02378600 & -1.83318400 & 0.63932300 \\
\hline
\end{tabular}

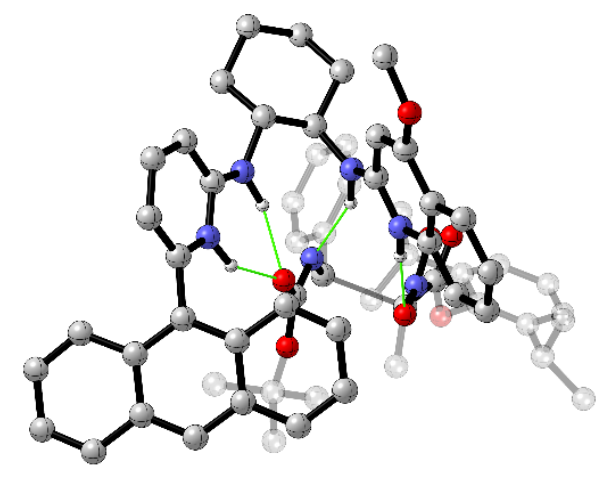

- Thermochemistry -

Zero-point correction= 1.224266 (Hartree/Particle)

Thermal correction to Energy= 1.292523 
Thermal correction to Enthalpy $=\quad 1.293468$

Thermal correction to Gibbs Free Energy= $\quad 1.120286$

Sum of electronic and zero-point Energies $=\quad-3256.480515$

Sum of electronic and thermal Energies $=\quad-3256.412258$

Sum of electronic and thermal Enthalpies $=\quad-3256.411313$

Sum of electronic and thermal Free Energies $=\quad-3256.584495$

Number of Imaginary Frequencies $=1$

E (Single Point Energy) $\left[\mathrm{SMD}_{\text {(Toluene) }} \mathrm{M} 06-2 \mathrm{X} / 6-311++\mathrm{G}(2 \mathrm{~d}, 2 \mathrm{p})\right]=-3258.781579$

$\begin{array}{lrrr}\mathrm{O} & 4.60470100 & 0.54207000 & 0.52195800 \\ \mathrm{C} & 4.29368200 & -0.57929200 & 0.22149100 \\ \mathrm{O} & 5.20344700 & -1.53181400 & -0.13273200 \\ \mathrm{C} & 2.92449600 & -1.17114900 & 0.17273300 \\ \mathrm{C} & 2.79328800 & -2.66231400 & 0.00584200 \\ \mathrm{H} & 3.15219800 & -3.20219800 & 0.88947700 \\ \mathrm{H} & 3.39715900 & -2.98028000 & -0.84613400 \\ \mathrm{H} & 1.75219800 & -2.92657000 & -0.16928200 \\ \mathrm{~N} & 2.00786500 & -0.57879500 & 1.03786100 \\ \mathrm{O} & 0.97201700 & -1.22844800 & 1.33551600 \\ \mathrm{O} & 2.09056500 & 0.60716300 & 1.36813700 \\ \mathrm{C} & 1.84129700 & -0.39214400 & -1.58224000 \\ \mathrm{C} & 2.77995100 & 0.60726800 & -2.13568200 \\ \mathrm{C} & 3.41056700 & 0.32860200 & -3.35023800 \\ \mathrm{C} & 2.97966600 & 1.85019000 & -1.51956500 \\ \mathrm{C} & 4.19155600 & 1.29885700 & -3.97378000 \\ \mathrm{C} & 3.78653300 & 2.80284400 & -2.12907600 \\ \mathrm{C} & 4.37947800 & 2.53603900 & -3.36440200 \\ \mathrm{C} & -0.38602700 & -0.78882700 & -0.99117900 \\ \mathrm{O} & -1.48724200 & -0.35603700 & -0.60258200 \\ \mathrm{O} & -0.18662800 & -2.06018900 & -1.32467100 \\ \mathrm{C} & -1.19048700 & -3.10002100 & -1.09193900 \\ \mathrm{C} & -2.40281300 & -2.87386800 & -1.98878600 \\ \mathrm{H} & -2.98617000 & -2.00929500 & -1.67117700 \\ \mathrm{H} & -3.05546700 & -3.75143200 & -1.94331300 \\ \mathrm{H} & -2.08772400 & -2.73440800 & -3.02739600 \\ \mathrm{C} & -1.55059600 & -3.15751600 & 0.38880500 \\ \mathrm{H} & -0.63988000 & -3.24670300 & 0.98925500\end{array}$




$\begin{array}{lrrr}\mathrm{H} & -2.17883600 & -4.03612300 & 0.56728400 \\ \mathrm{H} & -2.09989300 & -2.26811400 & 0.70799100 \\ \mathrm{C} & -0.46237400 & -4.37040200 & -1.51877500 \\ \mathrm{H} & 0.41111400 & -4.55059800 & -0.88664100 \\ \mathrm{H} & -0.13222600 & -4.29091000 & -2.55841900 \\ \mathrm{H} & -1.13405100 & -5.22861800 & -1.43086400 \\ \mathrm{~N} & 0.66019500 & 0.06968500 & -1.15507000 \\ \mathrm{H} & 1.89595400 & -1.39277800 & -2.01614400 \\ \mathrm{H} & 5.00203900 & 3.28668600 & -3.84161000 \\ \mathrm{H} & 4.66011400 & 1.08369300 & -4.92949800 \\ \mathrm{H} & 3.96748400 & 3.75231400 & -1.63414600 \\ \mathrm{H} & 2.55380100 & 2.03157100 & -0.53651700 \\ \mathrm{H} & 3.26922600 & -0.64152300 & -3.82041900 \\ \mathrm{C} & 0.56552100 & 5.57508700 & -1.46832700 \\ \mathrm{C} & -0.81235400 & 5.76545500 & -2.10981000 \\ \mathrm{C} & -1.47814100 & 4.42073600 & -2.42105400 \\ \mathrm{C} & -1.58968900 & 3.58035100 & -1.14385500 \\ \mathrm{C} & -0.17064400 & 3.35636000 & -0.58221700 \\ \mathrm{C} & 0.48845300 & 4.68881900 & -0.22046200 \\ \mathrm{H} & -0.88429200 & 3.85898900 & -3.15271900 \\ \mathrm{H} & -0.72632600 & 6.35579500 & -3.02644000 \\ \mathrm{H} & -1.45591200 & 6.33762800 & -1.42677200 \\ \mathrm{H} & 1.00199200 & 6.54474500 & -1.21216600 \\ \mathrm{H} & -24170800 & 5.10183400 & -2.19273900 \\ \mathrm{H} & -2.82186200 & 1.31098700 & 5.16897600 \\ \mathrm{H} & -2.19772800 & 4.13552600 & -0.41302000 \\ \mathrm{H} & 0.41148900 & 2.92100100 & -1.40717500 \\ \mathrm{H} & 1.48640800 & 4.48783900 & 0.18139500 \\ \mathrm{H} & -0.08018200 & 5.19873900 & 0.56921700 \\ \mathrm{H} & -2.46746700 & 4.57159700 & -2.86663800 \\ \mathrm{~N} & -2.19765000 & 2.27202400 & -1.34372400 \\ \mathrm{H} & -1.61265800 & 1.44951300 & -1.14646300 \\ \mathrm{~N} & -0.08654400 & 2.31826900 & 0.43719000 \\ \mathrm{H} & 0.41955000 & 1.48264900 & 0.10014700 \\ \mathrm{C} & -0.79047200 & 2.16337100 & 1.55800500 \\ \mathrm{C} & -1.64166200 & 3.15872400 & 2.12234300 \\ \mathrm{H} & -1.31740400 & 0.67947500 & 3.36968800 \\ \mathrm{H} & -0.04346000 & 0.25993300 & 1.80783900 \\ \mathrm{H} & -2.32328200 & 2.89122800 & 3.28411000 \\ \mathrm{H} & -1.69321100 & 4.12437300 & 1.64398600 \\ \mathrm{H} & -1.08759400 & -0.56236300 & 3.98660900 \\ \mathrm{H} & -17877600 & 1.62180800 & 3.95473500 \\ \mathrm{H} & -1430400 & 3.75017400 & 3.89017600 \\ \mathrm{H} & -0.09238400 & 5.77400300\end{array}$




\begin{tabular}{|c|c|c|c|}
\hline $\mathrm{H}$ & -1.53920700 & -1.79131000 & 5.66987500 \\
\hline $\mathrm{H}$ & -3.48107800 & 2.04600400 & 5.61665000 \\
\hline $\mathrm{H}$ & -3.08056000 & -0.14567700 & 6.71357700 \\
\hline $\mathrm{N}$ & -0.68615800 & 0.97696500 & 2.18446700 \\
\hline $\mathrm{C}$ & -3.51398600 & 2.04651600 & -1.33313100 \\
\hline $\mathrm{C}$ & -4.52389500 & 3.02950300 & -1.46114100 \\
\hline $\mathrm{C}$ & -5.21599100 & 0.34747200 & -1.15066200 \\
\hline $\mathrm{H}$ & -3.17354700 & 0.05697300 & -1.02261100 \\
\hline $\mathrm{C}$ & -5.84213900 & 2.63520400 & -1.45649300 \\
\hline $\mathrm{H}$ & -4.24574800 & 4.06823300 & -1.58157000 \\
\hline $\mathrm{C}$ & -6.20938400 & 1.28414600 & -1.30575900 \\
\hline $\mathrm{H}$ & -6.61761300 & 3.38743800 & -1.56292500 \\
\hline $\mathrm{H}$ & -7.24578400 & 0.97251100 & -1.27768400 \\
\hline $\mathrm{N}$ & -3.91738700 & 0.75677000 & -1.18550800 \\
\hline $\mathrm{C}$ & -5.41796200 & -1.09446800 & -0.87731700 \\
\hline $\mathrm{C}$ & -5.93166500 & -1.94124400 & -1.87797700 \\
\hline $\mathrm{C}$ & -5.03459500 & -1.58549900 & 0.38765100 \\
\hline $\mathrm{C}$ & -6.30098700 & -1.48213100 & -3.18272300 \\
\hline $\mathrm{C}$ & -6.05946000 & -3.34294700 & -1.59226000 \\
\hline $\mathrm{C}$ & -5.16053700 & -2.99080900 & 0.64990600 \\
\hline $\mathrm{C}$ & -4.56269600 & -0.74266800 & 1.44711100 \\
\hline $\mathrm{C}$ & -6.78475500 & -2.35020300 & -4.11744200 \\
\hline $\mathrm{H}$ & -6.18932000 & -0.43109500 & -3.42877800 \\
\hline $\mathrm{C}$ & -6.57341000 & -4.21707000 & -2.60096400 \\
\hline $\mathrm{C}$ & -5.66503300 & -3.82925500 & -0.34487400 \\
\hline $\mathrm{C}$ & -4.78016800 & -3.50015900 & 1.93205100 \\
\hline $\mathrm{H}$ & -4.49641400 & 0.33066600 & 1.29495500 \\
\hline $\mathrm{C}$ & -4.22768500 & -1.26585100 & 2.66130100 \\
\hline $\mathrm{C}$ & -6.92995400 & -3.73803300 & -3.82521200 \\
\hline $\mathrm{H}$ & -7.06053600 & -1.98349900 & -5.10094800 \\
\hline $\mathrm{H}$ & -6.67072600 & -5.27307200 & -2.36528400 \\
\hline $\mathrm{H}$ & -5.76064500 & -4.89352500 & -0.13981200 \\
\hline $\mathrm{C}$ & -4.32140800 & -2.66701700 & 2.90728500 \\
\hline $\mathrm{H}$ & -4.87555700 & -4.56802600 & 2.10902600 \\
\hline $\mathrm{H}$ & -3.90159900 & -0.60706400 & 3.45991000 \\
\hline $\mathrm{H}$ & -7.31969300 & -4.40665400 & -4.58527200 \\
\hline $\mathrm{H}$ & -4.03937500 & -3.05740700 & 3.87974500 \\
\hline $\mathrm{C}$ & 9.19626500 & -0.86245300 & 0.76939300 \\
\hline $\mathrm{C}$ & 8.67140500 & -0.37161400 & -0.42187500 \\
\hline $\mathrm{C}$ & 7.32837200 & -0.56268500 & -0.74977800 \\
\hline $\mathrm{C}$ & 6.54792700 & -1.26471800 & 0.17079400 \\
\hline $\mathrm{C}$ & 7.03714500 & -1.77797700 & 1.37398500 \\
\hline $\mathrm{C}$ & 8.38740200 & -1.56069600 & 1.65675200 \\
\hline $\mathrm{H}$ & 10.24371900 & -0.70144700 & 1.00415200 \\
\hline $\mathrm{H}$ & 9.31750600 & 0.17272600 & -1.10207600 \\
\hline $\mathrm{C}$ & 6.72282600 & -0.08378300 & -2.0563150 \\
\hline
\end{tabular}




$\begin{array}{lrrr}\mathrm{C} & 6.11686900 & -2.50808900 & 2.33607400 \\ \mathrm{H} & 8.81049200 & -1.94524800 & 2.57935600 \\ \mathrm{C} & 7.57123000 & 0.97373000 & -2.76044400 \\ \mathrm{H} & 5.75358800 & 0.37400800 & -1.81945400 \\ \mathrm{C} & 6.48499000 & -1.27710400 & -2.99293800 \\ \mathrm{H} & 6.05081700 & -0.94121800 & -3.94104700 \\ \mathrm{H} & 5.80912500 & -2.00945400 & -2.54198400 \\ \mathrm{H} & 7.43414800 & -1.77757700 & -3.21353100 \\ \mathrm{H} & 7.03998800 & 1.34395400 & -3.64237100 \\ \mathrm{H} & 8.52470000 & 0.55838100 & -3.10491100 \\ \mathrm{H} & 7.78093000 & 1.82475500 & -2.10511400 \\ \mathrm{C} & 5.43175700 & -1.51341600 & 3.28637000 \\ \mathrm{C} & 6.83242400 & -3.59671100 & 3.13892300 \\ \mathrm{H} & 4.72413900 & -2.03393400 & 3.94096100 \\ \mathrm{H} & 4.89708900 & -0.72485800 & 2.74875400 \\ \mathrm{H} & 6.18285200 & -1.02573500 & 3.91753600 \\ \mathrm{H} & 7.53227000 & -3.16651300 & 3.86284600 \\ \mathrm{H} & 7.38931500 & -4.27643800 & 2.48750100 \\ \mathrm{H} & 6.10212400 & -4.18268100 & 3.70529300 \\ \mathrm{C} & -3.31593800 & 5.03923300 & 3.31931100 \\ \mathrm{H} & -3.73038800 & 4.95764000 & 2.30842100 \\ \mathrm{H} & -2.36491400 & 5.58038800 & 3.29290900 \\ \mathrm{H} & -4.01921800 & 5.55861700 & 3.96700100 \\ \mathrm{H} & 5.34230900 & -2.99535900 & 1.73296100\end{array}$

\section{Structure syn-(2S,3R)-TS2}

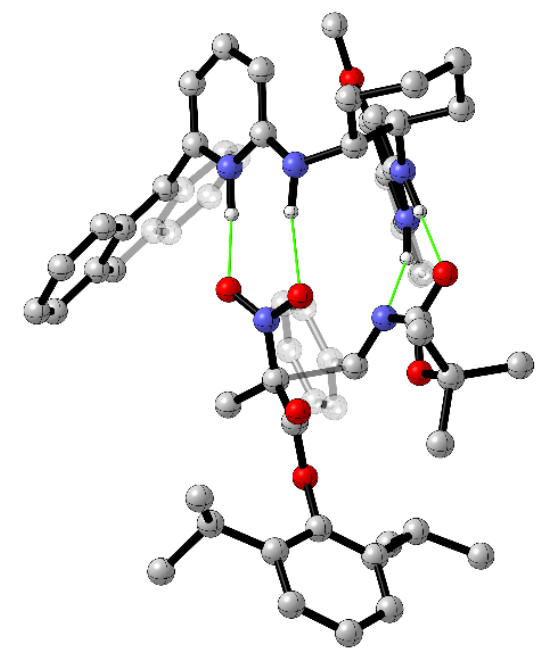

- Thermochemistry -

Zero-point correction=

1.224269 (Hartree/Particle) 


$\begin{array}{ll}\text { Thermal correction to Energy= } & 1.291714 \\ \text { Thermal correction to Enthalpy= } & 1.292659 \\ \text { Thermal correction to Gibbs Free Energy= } & 1.123500 \\ \text { Sum of electronic and zero-point Energies= } & -3256.490186 \\ \text { Sum of electronic and thermal Energies= } & -3256.422741 \\ \text { Sum of electronic and thermal Enthalpies= } & -3256.421797 \\ \text { Sum of electronic and thermal Free Energies= } & -3256.590956\end{array}$

Number of Imaginary Frequencies $=1$

E (Single Point Energy) [SMD(Toluene)M06-2X/6-311++G(2d,2p)] = -3258.785871

$\begin{array}{lrrr}\mathrm{O} & -3.69360900 & 0.36844000 & -1.43504500 \\ \mathrm{C} & -3.31088700 & -0.43992600 & -0.63396400 \\ \mathrm{O} & -4.11692200 & -1.11656700 & 0.22478900 \\ \mathrm{C} & -1.91624800 & -0.95451800 & -0.44066700 \\ \mathrm{C} & -1.79058600 & -2.46061600 & -0.41085300 \\ \mathrm{H} & -2.08673500 & -2.89026500 & -1.37545000 \\ \mathrm{H} & -2.44711700 & -2.85922800 & 0.36388200 \\ \mathrm{H} & -0.76459300 & -2.76324500 & -0.20498900 \\ \mathrm{~N} & -0.94787600 & -0.30098200 & -1.20250500 \\ \mathrm{O} & -1.02289600 & 0.91899800 & -1.38362800 \\ \mathrm{O} & 0.06810800 & -0.95119700 & -1.55423500 \\ \mathrm{C} & -1.36792800 & -0.16626900 & 1.41303000 \\ \mathrm{C} & -0.49711200 & -1.25907100 & 1.92230400 \\ \mathrm{C} & -0.98938800 & -2.14480900 & 2.88305500 \\ \mathrm{C} & 0.82075900 & -1.40405000 & 1.46965600 \\ \mathrm{C} & -0.17114300 & -3.14362200 & 3.40713600 \\ \mathrm{C} & 1.63568000 & -2.40023800 & 1.99220500 \\ \mathrm{C} & 1.14273300 & -3.27010200 & 2.96388100 \\ \mathrm{C} & -1.49546200 & 2.15524400 & 0.98643300 \\ \mathrm{O} & -0.92373500 & 3.24339100 & 0.84384600 \\ \mathrm{O} & -2.81663700 & 2.02094900 & 0.89514500 \\ \mathrm{C} & -3.63985600 & 3.17235500 & 0.47237200 \\ \mathrm{C} & -3.19935200 & 3.63870200 & -0.91326000 \\ \mathrm{H} & -3.96654600 & 4.30451900 & -1.32021500 \\ \mathrm{H} & -3.10214400 & 2.77235200 & -1.57422700 \\ \mathrm{H} & -2.25440900 & 4.18256200 & -0.87202400\end{array}$




\begin{tabular}{|c|c|c|c|}
\hline $\mathrm{C}$ & -3.56665200 & 4.27033400 & 1.52929900 \\
\hline $\mathrm{H}$ & -2.57213500 & 4.71113300 & 1.58905400 \\
\hline $\mathrm{H}$ & -3.84186800 & 3.86446300 & 2.50839200 \\
\hline $\mathrm{H}$ & -4.28687700 & 5.05377700 & 1.27404800 \\
\hline $\mathrm{C}$ & -5.05483900 & 2.61436100 & 0.40592800 \\
\hline $\mathrm{H}$ & -5.73884400 & 3.41634700 & 0.11317100 \\
\hline $\mathrm{H}$ & -5.36919900 & 2.24381500 & 1.38464700 \\
\hline $\mathrm{H}$ & -5.12891500 & 1.80673200 & -0.32531200 \\
\hline $\mathrm{N}$ & -0.76971700 & 1.02811700 & 1.26740600 \\
\hline $\mathrm{H}$ & -2.41119100 & -0.21498400 & 1.73736800 \\
\hline $\mathrm{H}$ & 1.78744200 & -4.04168500 & 3.37429300 \\
\hline $\mathrm{H}$ & -0.56195600 & -3.81960700 & 4.16113800 \\
\hline $\mathrm{H}$ & 2.66775900 & -2.48030600 & 1.66730600 \\
\hline $\mathrm{H}$ & 1.20185500 & -0.72763100 & 0.70936500 \\
\hline $\mathrm{H}$ & -2.01458400 & -2.04174800 & 3.22835900 \\
\hline $\mathrm{C}$ & 1.19156100 & 6.02431200 & -2.64724000 \\
\hline $\mathrm{C}$ & 2.06427700 & 6.36435000 & -1.43737900 \\
\hline $\mathrm{C}$ & 1.83045200 & 5.37377000 & -0.29499200 \\
\hline $\mathrm{C}$ & 2.08750900 & 3.92858200 & -0.74187800 \\
\hline $\mathrm{C}$ & 1.23625900 & 3.60418500 & -1.98856000 \\
\hline $\mathrm{C}$ & 1.46364200 & 4.59738700 & -3.13096200 \\
\hline $\mathrm{H}$ & 0.79020800 & 5.43919900 & 0.05185000 \\
\hline $\mathrm{H}$ & 1.85769700 & 7.38069500 & -1.09002300 \\
\hline $\mathrm{H}$ & 3.12254000 & 6.34179800 & -1.73342800 \\
\hline $\mathrm{H}$ & 1.37016200 & 6.73192900 & -3.46185900 \\
\hline $\mathrm{H}$ & 0.13308400 & 6.11694800 & -2.37052100 \\
\hline $\mathrm{H}$ & 3.15013500 & 3.81030700 & -0.98014100 \\
\hline $\mathrm{H}$ & 0.80975300 & 4.32774500 & -3.96549600 \\
\hline $\mathrm{H}$ & 2.49454600 & 4.53837200 & -3.50163200 \\
\hline $\mathrm{H}$ & 2.47002100 & 5.59815600 & 0.56475300 \\
\hline $\mathrm{N}$ & 1.71860800 & 3.00151300 & 0.31414700 \\
\hline $\mathrm{H}$ & 0.71004300 & 3.05619000 & 0.58339600 \\
\hline $\mathrm{N}$ & 1.35511000 & 2.19141600 & -2.36416600 \\
\hline $\mathrm{H}$ & 0.53615500 & 1.63406500 & -2.09340700 \\
\hline $\mathrm{C}$ & 2.50845400 & 1.51649400 & -2.46219700 \\
\hline $\mathrm{C}$ & 3.76786300 & 2.05216600 & -2.82558400 \\
\hline $\mathrm{C}$ & 3.51861700 & -0.66351200 & -2.25955000 \\
\hline $\mathrm{H}$ & 1.52871800 & -0.21467900 & -1.92189600 \\
\hline $\mathrm{C}$ & 4.85399200 & 1.21055500 & -2.91274900 \\
\hline $\mathrm{H}$ & 3.85097700 & 3.09985700 & -3.07689300 \\
\hline $\mathrm{C}$ & 4.74799600 & -0.16112500 & -2.62508900 \\
\hline $\mathrm{N}$ & 2.45742200 & 0.18508300 & -2.20023500 \\
\hline $\mathrm{C}$ & 2.49176600 & 2.22744200 & 1.07040300 \\
\hline $\mathrm{C}$ & 3.90069800 & 2.05688800 & 0.90675300 \\
\hline $\mathrm{C}$ & 2.52087600 & 0.77752200 & 2.98317000 \\
\hline $\mathrm{C}$ & 4.57762200 & 1.23405900 & 1.77300100 \\
\hline
\end{tabular}




\begin{tabular}{|c|c|c|c|}
\hline $\mathrm{H}$ & 4.40294100 & 2.56654500 & 0.09738800 \\
\hline $\mathrm{C}$ & 3.90783800 & 0.59157600 & 2.87705300 \\
\hline $\mathrm{N}$ & 1.86084400 & 1.54681100 & 2.04960100 \\
\hline $\mathrm{C}$ & 1.80150700 & 0.17858800 & 4.02987700 \\
\hline $\mathrm{C}$ & 2.47188200 & -0.60466900 & 4.94398400 \\
\hline $\mathrm{C}$ & 3.86295500 & -0.79877500 & 4.84806400 \\
\hline $\mathrm{C}$ & -8.23375200 & -0.58007300 & 0.19568400 \\
\hline $\mathrm{C}$ & -7.60777400 & -1.09035600 & -0.93116400 \\
\hline $\mathrm{C}$ & -6.22344000 & -1.29281300 & -0.96690500 \\
\hline $\mathrm{C}$ & -5.50546800 & -0.92732700 & 0.17163100 \\
\hline $\mathrm{C}$ & -6.10461200 & -0.44650100 & 1.34469600 \\
\hline $\mathrm{C}$ & -7.48696300 & -0.27550900 & 1.33130000 \\
\hline $\mathrm{H}$ & -9.30875900 & -0.43147800 & 0.19927400 \\
\hline $\mathrm{H}$ & -8.20049300 & -1.34884000 & -1.80437500 \\
\hline $\mathrm{C}$ & -5.58815900 & -1.93138000 & -2.18966700 \\
\hline $\mathrm{C}$ & -5.25691500 & -0.17317400 & 2.57572600 \\
\hline $\mathrm{H}$ & -7.99030500 & 0.10386600 & 2.21399800 \\
\hline $\mathrm{C}$ & -6.17383300 & -3.32823700 & -2.43394800 \\
\hline $\mathrm{H}$ & -4.51762600 & -2.06154800 & -2.00165300 \\
\hline $\mathrm{C}$ & -5.74818100 & -1.04409400 & -3.42859900 \\
\hline $\mathrm{H}$ & -5.25487200 & -1.50320600 & -4.29147100 \\
\hline $\mathrm{H}$ & -5.31313500 & -0.05706000 & -3.25798000 \\
\hline $\mathrm{H}$ & -6.80719700 & -0.91902700 & -3.67897000 \\
\hline $\mathrm{H}$ & -6.05484600 & -3.96832900 & -1.55498100 \\
\hline $\mathrm{H}$ & -5.67210600 & -3.80553900 & -3.28178700 \\
\hline $\mathrm{H}$ & -7.24170500 & -3.27354300 & -2.66861500 \\
\hline $\mathrm{H}$ & -4.37465800 & 0.39065100 & 2.24346800 \\
\hline $\mathrm{C}$ & -4.78296400 & -1.49686100 & 3.19481200 \\
\hline $\mathrm{C}$ & -5.97081600 & 0.66117500 & 3.63993200 \\
\hline $\mathrm{H}$ & -4.14301900 & -1.30540800 & 4.06430100 \\
\hline $\mathrm{H}$ & -4.22681700 & -2.10140900 & 2.47221400 \\
\hline $\mathrm{H}$ & -5.64363800 & -2.08290200 & 3.53408800 \\
\hline $\mathrm{H}$ & -6.79668700 & 0.10226800 & 4.09297500 \\
\hline $\mathrm{H}$ & -6.37707400 & 1.59277000 & 3.23374200 \\
\hline $\mathrm{H}$ & -5.27069600 & 0.91638300 & 4.44090500 \\
\hline $\mathrm{H}$ & 0.18852900 & 3.69884300 & -1.67195100 \\
\hline $\mathrm{H}$ & 0.81473200 & 1.42363300 & 1.92971700 \\
\hline $\mathrm{O}$ & 5.88195400 & 0.95785700 & 1.68148800 \\
\hline $\mathrm{C}$ & 6.63174500 & 1.56354100 & 0.63847200 \\
\hline $\mathrm{H}$ & 6.23148200 & 1.27290800 & -0.34025000 \\
\hline $\mathrm{H}$ & 6.61934600 & 2.65348800 & 0.73825400 \\
\hline $\mathrm{H}$ & 7.64899000 & 1.19256500 & 0.74824800 \\
\hline $\mathrm{C}$ & 4.57476100 & -0.20370400 & 3.82935000 \\
\hline $\mathrm{H}$ & 0.72721900 & 0.32471600 & 4.08742000 \\
\hline $\mathrm{H}$ & 1.91629300 & -1.07683600 & 5.74754200 \\
\hline $\mathrm{H}$ & 4.37501300 & -1.40826200 & 5.58439100 \\
\hline
\end{tabular}




$\begin{array}{lrrr}\mathrm{H} & 5.64972700 & -0.32312500 & 3.75397900 \\ \mathrm{C} & 3.25775000 & -2.06978600 & -1.87665900 \\ \mathrm{C} & 2.34718500 & -2.84161400 & -2.62701800 \\ \mathrm{C} & 3.90987700 & -2.59335500 & -0.74047200 \\ \mathrm{C} & 1.69778200 & -2.36176100 & -3.80952600 \\ \mathrm{C} & 2.07226600 & -4.18634800 & -2.20764000 \\ \mathrm{C} & 3.64883100 & -3.95358600 & -0.36180400 \\ \mathrm{C} & 4.78908700 & -1.82701300 & 0.09319000 \\ \mathrm{C} & 0.81409700 & -3.14624600 & -4.48993800 \\ \mathrm{H} & 1.91846300 & -1.36501700 & -4.17783800 \\ \mathrm{C} & 1.13399300 & -4.97351200 & -2.94646000 \\ \mathrm{C} & 2.73396600 & -4.70759800 & -1.09643200 \\ \mathrm{C} & 4.30632000 & -4.50130900 & 0.78494000 \\ \mathrm{H} & 4.96665300 & -0.78173200 & -0.13709100 \\ \mathrm{C} & 5.39314000 & -2.38446700 & 1.18287100 \\ \mathrm{C} & 0.51579100 & -4.46887000 & -4.04981300 \\ \mathrm{H} & 0.33381200 & -2.76334800 & -5.38457000 \\ \mathrm{H} & 0.93404200 & -5.98610500 & -2.60781600 \\ \mathrm{H} & 2.52904000 & -5.73163600 & -0.79219700 \\ \mathrm{C} & 5.15854900 & -3.74602500 & 1.53442400 \\ \mathrm{H} & 4.10230400 & -5.53604200 & 1.04619000 \\ \mathrm{H} & 6.05392300 & -1.77618600 & 1.79250500 \\ \mathrm{H} & -0.19292500 & -5.07150500 & -4.60778700 \\ \mathrm{H} & 5.65334100 & -4.17012300 & 2.40176800 \\ \mathrm{H} & 5.59823200 & -0.82854400 & -2.69079900 \\ \mathrm{H} & 5.81116200 & 1.61726000 & -3.22632100\end{array}$




\section{Optimized Precomplex Structures Using the $\mathrm{C}_{1}-$ Symmetric Catalyst}

Table S2. Single point energies of all structures calculated at the $\mathrm{SMD}_{\text {(Toluene) }} \mathrm{M} 06-2 \mathrm{X} / 6$ $311++\mathrm{G}(2 \mathrm{~d}, 2 \mathrm{p})$ level of theory and thermal correction to Gibbs Free Energy and Gibbs Free Energy calculated at the M06-2X/6-31G(d) level of theory. All energies are reported in Hartrees.

\begin{tabular}{|c|c|c|c|c|}
\hline Structure & $\begin{array}{c}\text { Single Point } \\
\text { Energies (E) } \\
\text { SMD }_{\text {(Toluene) M06- }} \\
\text { 2X/6- } \\
\text { 311++G(2d,2p) }\end{array}$ & $\begin{array}{c}\text { Thermal } \\
\text { Corrections } \\
\text { to Gibbs Free } \\
\text { Energies (G) } \\
\text { M06-2X/6- } \\
\text { 31G(d) }\end{array}$ & $\begin{array}{c}\text { Gibbs Free } \\
\text { Energies (G) } \\
\text { M06-2X/6- } \\
\text { 31G(d) }\end{array}$ & $\begin{array}{c}\text { Gibbs Free Energies (G) } \\
\text { SMD }_{(\text {Toluene) }} \text { M06-2X/6- } \\
\text { 311++G(2d,2p)// M06- }_{\text {2X/6-31G(d) }}\end{array}$ \\
\hline $\begin{array}{c}\text { anti- }(2 R, 3 R)- \\
\text { TS } \\
\text { Precomplex }\end{array}$ & -3258.800595 & 1.114708 & -3256.607967 & -3257.685887 \\
\hline $\begin{array}{c}\text { syn-(2S,3R)- } \\
\text { TS } \\
\text { Precomplex } \\
\end{array}$ & -3258.79932 & 1.115466 & -3256.609059 & -3257.683854 \\
\hline $\begin{array}{c}\text { syn-(2R,3S)- } \\
\text { TS } \\
\text { Precomplex }\end{array}$ & -3258.803935 & 1.115626 & -3256.609983 & -3257.688309 \\
\hline $\begin{array}{c}\text { anti-(2S,3S)- } \\
\text { TS } \\
\text { Precomplex }\end{array}$ & -3258.813529 & 1.12113 & -3256.622811 & -3257.692399 \\
\hline $\begin{array}{c}\text { anti-(2R,3R)- } \\
\text { TS2 } \\
\text { Precomplex } \\
\end{array}$ & -3258.796537 & 1.116495 & -3256.607377 & -3257.680042 \\
\hline $\begin{array}{c}\text { syn- }(2 S, 3 R)- \\
\text { TS2 } \\
\text { Precomplex }\end{array}$ & -3258.803853 & 1.115434 & -3256.612920 & -3257.688419 \\
\hline $\begin{array}{l}\text { anti-(2R,3S)- } \\
\text { TS2 } \\
\text { Precomplex }\end{array}$ & -3258.798629 & 1.116957 & -3256.602929 & -3257.681672 \\
\hline $\begin{array}{c}\text { syn-(2S,3S)- } \\
\text { TS2 } \\
\text { Precomplex }\end{array}$ & -3258.798883 & 1.115461 & -3256.602169 & -3257.683422 \\
\hline
\end{tabular}




\section{Structure anti-(2R,3R)-TS Precomplex}

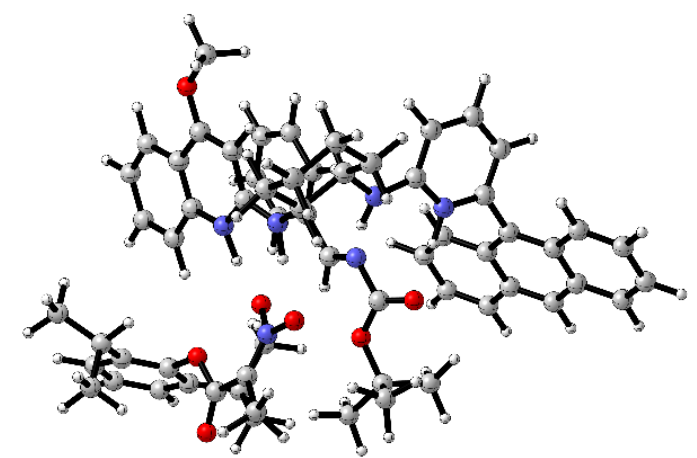

- Thermochemistry -

Zero-point correction $=$

1.222021 (Hartree/Particle)

Thermal correction to Energy=

1.291519

Thermal correction to Enthalpy=

1.292464

Thermal correction to Gibbs Free Energy=

1.114708

Sum of electronic and zero-point Energies=

$-3256.500654$

Sum of electronic and thermal Energies=

$-3256.431156$

Sum of electronic and thermal Enthalpies=

$-3256.430212$

Sum of electronic and thermal Free Energies=

$-3256.607967$

Number of Imaginary Frequencies $=0$

E (Single Point Energy) [SMD(Toluene) M06-2X/6-311++G(2d,2p)] = -3258.800595

$\begin{array}{llll}\mathrm{O} & -3.92746200 & -3.23761700 & 2.57695300 \\ \mathrm{C} & -3.42810600 & -2.27467700 & 2.04359900 \\ \mathrm{O} & -3.91568300 & -1.71910000 & 0.90909900 \\ \mathrm{C} & -2.27074700 & -1.58143400 & 2.64296700 \\ \mathrm{C} & -1.97854600 & -1.87569900 & 4.08123400 \\ \mathrm{H} & -2.91662600 & -1.98027200 & 4.63040500 \\ \mathrm{H} & -1.37886700 & -1.07468400 & 4.51247100 \\ \mathrm{H} & -1.43504200 & -2.82108800 & 4.19532700\end{array}$




\begin{tabular}{|c|c|c|c|}
\hline $\mathrm{N}$ & -1.49700700 & -0.75771900 & 1.96039300 \\
\hline $\mathrm{O}$ & -1.69587300 & -0.52530000 & 0.70165700 \\
\hline $\mathrm{O}$ & -0.50620100 & -0.15370600 & 2.50733400 \\
\hline $\mathrm{C}$ & 0.25337200 & -0.43279800 & -1.04408500 \\
\hline $\mathrm{C}$ & -0.23877800 & 0.53116800 & -2.02715900 \\
\hline $\mathrm{C}$ & -1.26201100 & 0.13396900 & -2.89449900 \\
\hline $\mathrm{C}$ & 0.29615900 & 1.82417900 & -2.12747400 \\
\hline $\mathrm{C}$ & -1.75659800 & 1.01908300 & -3.84875000 \\
\hline $\mathrm{C}$ & -0.19522200 & 2.70143100 & -3.08245500 \\
\hline $\mathrm{C}$ & -1.22196300 & 2.30016000 & -3.94332600 \\
\hline $\mathrm{C}$ & 1.60456200 & -1.16348200 & 0.66657400 \\
\hline $\mathrm{O}$ & 2.68323900 & -1.02829900 & 1.23300000 \\
\hline $\mathrm{O}$ & 0.81542900 & -2.20547000 & 0.76522400 \\
\hline $\mathrm{C}$ & 1.18815600 & -3.36732800 & 1.59168600 \\
\hline $\mathrm{C}$ & -0.05037900 & -4.24935300 & 1.49795800 \\
\hline $\mathrm{H}$ & -0.91947000 & -3.74681600 & 1.93490100 \\
\hline $\mathrm{H}$ & 0.12080000 & -5.18299900 & 2.04041100 \\
\hline $\mathrm{H}$ & -0.27327600 & -4.48962300 & 0.45366100 \\
\hline $\mathrm{C}$ & 1.43743200 & -2.92390200 & 3.03012700 \\
\hline $\mathrm{H}$ & 2.43045400 & -2.49090600 & 3.15245500 \\
\hline $\mathrm{H}$ & 1.34563400 & -3.79180800 & 3.69046000 \\
\hline $\mathrm{H}$ & 0.69216400 & -2.17716100 & 3.31970600 \\
\hline $\mathrm{C}$ & 2.39659500 & -4.04533500 & 0.95794400 \\
\hline $\mathrm{H}$ & 2.63192600 & -4.95450800 & 1.51963600 \\
\hline $\mathrm{H}$ & 3.27163100 & -3.39117700 & 0.97489200 \\
\hline $\mathrm{H}$ & 2.17847600 & -4.32396400 & -0.07782400 \\
\hline $\mathrm{N}$ & 1.15883200 & -0.13568300 & -0.18545100 \\
\hline $\mathrm{H}$ & -0.16488400 & -1.43986600 & -1.09718700 \\
\hline $\mathrm{H}$ & -1.60607900 & 2.99325700 & -4.68544400 \\
\hline $\mathrm{H}$ & -2.56061700 & 0.71264900 & -4.50956100 \\
\hline
\end{tabular}




\begin{tabular}{|c|c|c|c|}
\hline $\mathrm{H}$ & 0.21609900 & 3.70355000 & -3.16335100 \\
\hline $\mathrm{H}$ & 1.08705400 & 2.12279700 & -1.44335400 \\
\hline & -1.67813400 & -0.86646900 & -2.80358700 \\
\hline & 0.95977200 & 5.08494700 & 3.87714900 \\
\hline $\mathrm{C}$ & 2.21882500 & 5.48308200 & 3.10725200 \\
\hline $\mathrm{C}$ & 2.84255800 & 4.26434500 & 2.42578300 \\
\hline C & 1.85746500 & 3.59266700 & 1.45726300 \\
\hline C & 0.57414700 & 3.21616800 & 2.22759900 \\
\hline C & -0.05310500 & 4.41953000 & 2.94365700 \\
\hline H & 3.12193300 & 3.51826900 & 3.18105500 \\
\hline $\mathrm{H}$ & 2.95079500 & 5.94265000 & 3.77748700 \\
\hline $\mathrm{H}$ & 1.96402900 & 6.23955100 & 2.35166400 \\
\hline $\mathrm{H}$ & 0.50532200 & 5.95859400 & 4.35329200 \\
\hline $\mathrm{H}$ & 1.23071900 & 4.38888100 & 4.68129700 \\
\hline $\mathrm{H}$ & 1.60164500 & 4.28937000 & 0.64029100 \\
\hline $\mathrm{H}$ & -0.92914700 & 4.06697400 & 3.49660500 \\
\hline $\mathrm{H}$ & -0.41081400 & 5.16335900 & 2.22362400 \\
\hline $\mathrm{H}$ & 3.76442000 & 4.54452100 & 1.90714700 \\
\hline $\mathrm{N}$ & 2.40492200 & 2.35691300 & 0.90966800 \\
\hline $\mathrm{H}$ & 1.80149200 & 1.52906500 & 0.89538600 \\
\hline $\mathrm{N}$ & -0.35785000 & 2.42578800 & 1.43232600 \\
\hline $\mathrm{H}$ & -0.45817100 & 1.44135700 & 1.75188900 \\
\hline $\mathrm{C}$ & -1.26020000 & 2.84792000 & 0.52852800 \\
\hline C & -1.30356700 & 4.14358700 & -0.06132200 \\
\hline $\mathrm{C}$ & -3.11710700 & 2.15857300 & -0.81399900 \\
\hline $\mathrm{H}$ & -2.02983500 & 0.92052300 & 0.47559100 \\
\hline C & -2.25246000 & 4.41306600 & -1.02099800 \\
\hline $\mathrm{H}$ & -0.56293700 & 4.87609900 & 0.21752700 \\
\hline C & -3.99119500 & 1.11339500 & -1.16635400 \\
\hline $\mathrm{C}$ & -3.20630900 & 3.41556800 & -1.43137100 \\
\hline
\end{tabular}




\begin{tabular}{|c|c|c|c|}
\hline $\mathrm{O}$ & -2.35990400 & 5.57972800 & -1.65905400 \\
\hline $\mathrm{C}$ & -4.93778500 & 1.34337700 & -2.14165600 \\
\hline $\mathrm{H}$ & -3.89592300 & 0.15104900 & -0.66604000 \\
\hline $\mathrm{C}$ & -4.17855000 & 3.62379800 & -2.42987200 \\
\hline $\mathrm{C}$ & -5.03095300 & 2.59793600 & -2.77941200 \\
\hline $\mathrm{H}$ & -5.61971200 & 0.54323400 & -2.41502400 \\
\hline $\mathrm{H}$ & -4.24350400 & 4.59495400 & -2.90766500 \\
\hline $\mathrm{H}$ & -5.78410800 & 2.75771600 & -3.54335400 \\
\hline $\mathrm{N}$ & -2.16390900 & 1.93711800 & 0.14731600 \\
\hline $\mathrm{C}$ & 3.52148800 & 2.26260600 & 0.16735500 \\
\hline $\mathrm{C}$ & 4.21781200 & 3.34031700 & -0.42015500 \\
\hline $\mathrm{C}$ & 5.11619500 & 0.72806400 & -0.77559400 \\
\hline $\mathrm{C}$ & 5.34462000 & 3.07771100 & -1.16979000 \\
\hline $\mathrm{H}$ & 3.84135900 & 4.34811200 & -0.30260800 \\
\hline $\mathrm{C}$ & 5.80838500 & 1.76648700 & -1.36069500 \\
\hline $\mathrm{H}$ & 5.87191200 & 3.90294100 & -1.63818900 \\
\hline $\mathrm{H}$ & 6.68541000 & 1.55139300 & -1.95813600 \\
\hline $\mathrm{N}$ & 4.01588800 & 1.01416500 & -0.03085900 \\
\hline $\mathrm{C}$ & 5.48926200 & -0.70356500 & -0.88653800 \\
\hline $\mathrm{C}$ & 4.62044100 & -1.59124100 & -1.55530800 \\
\hline $\mathrm{C}$ & 6.70049600 & -1.14712900 & -0.31767300 \\
\hline $\mathrm{C}$ & 3.40381600 & -1.17420900 & -2.18518500 \\
\hline $\mathrm{C}$ & 4.98170600 & -2.97720200 & -1.64823100 \\
\hline $\mathrm{C}$ & 7.04823900 & -2.53650800 & -0.43148900 \\
\hline $\mathrm{C}$ & 7.59666100 & -0.29092800 & 0.40123700 \\
\hline $\mathrm{C}$ & 2.59953100 & -2.07347500 & -2.82411200 \\
\hline $\mathrm{H}$ & 3.11430000 & -0.12781500 & -2.16017800 \\
\hline $\mathrm{C}$ & 4.11192500 & -3.88586000 & -2.32894800 \\
\hline $\mathrm{C}$ & 6.18216500 & -3.41142200 & -1.08567900 \\
\hline $\mathrm{C}$ & 8.27557500 & -3.00207100 & 0.13715400 \\
\hline
\end{tabular}




\begin{tabular}{|c|c|c|c|}
\hline $\mathrm{H}$ & 7.34383000 & 0.75537900 & 0.53437800 \\
\hline $\mathrm{C}$ & 8.75600400 & -0.77589500 & 0.93166400 \\
\hline $\mathrm{C}$ & 2.95135100 & -3.45253300 & -2.89705100 \\
\hline $\mathrm{H}$ & 1.68407700 & -1.72936400 & -3.29872800 \\
\hline $\mathrm{H}$ & 4.40432200 & -4.93076000 & -2.38545400 \\
\hline $\mathrm{H}$ & 6.45255500 & -4.46221400 & -1.16339300 \\
\hline $\mathrm{C}$ & 9.10974900 & -2.14995100 & 0.79412600 \\
\hline $\mathrm{H}$ & 8.52217100 & -4.05485500 & 0.03291700 \\
\hline $\mathrm{H}$ & 9.41894200 & -0.10914600 & 1.47376700 \\
\hline $\mathrm{H}$ & 2.29876300 & -4.14686700 & -3.41595000 \\
\hline $\mathrm{H}$ & 10.03896900 & -2.50966600 & 1.22281000 \\
\hline $\mathrm{C}$ & -7.12548800 & -3.28654600 & -1.17815000 \\
\hline $\mathrm{C}$ & -5.89207800 & -3.92936200 & -1.23549900 \\
\hline $\mathrm{C}$ & -4.79555300 & -3.44839700 & -0.51875800 \\
\hline $\mathrm{C}$ & -5.00547700 & -2.30906000 & 0.26533500 \\
\hline $\mathrm{C}$ & -6.22597200 & -1.63496800 & 0.34910500 \\
\hline $\mathrm{C}$ & -7.28966400 & -2.14909600 & -0.39688900 \\
\hline $\mathrm{H}$ & -7.96360800 & -3.67797300 & -1.74601600 \\
\hline $\mathrm{H}$ & -5.78494500 & -4.81784300 & -1.84851500 \\
\hline $\mathrm{C}$ & -3.41439700 & -4.07820800 & -0.60534400 \\
\hline $\mathrm{C}$ & -6.37504800 & -0.44018700 & 1.27396100 \\
\hline $\mathrm{H}$ & -8.25731600 & -1.65868700 & -0.36004300 \\
\hline $\mathrm{C}$ & -3.42292100 & -5.47744000 & -1.21917300 \\
\hline $\mathrm{H}$ & -3.03217500 & -4.17540700 & 0.41799400 \\
\hline $\mathrm{C}$ & -2.46290600 & -3.15834400 & -1.38756500 \\
\hline $\mathrm{H}$ & -1.46934600 & -3.61708300 & -1.47118400 \\
\hline $\mathrm{H}$ & -2.35678600 & -2.19035200 & -0.88695700 \\
\hline $\mathrm{H}$ & -2.84893600 & -3.00385300 & -2.40311400 \\
\hline $\mathrm{H}$ & -3.69491100 & -5.44808200 & -2.28016700 \\
\hline $\mathrm{H}$ & -4.12195100 & -6.14092200 & -0.70212700 \\
\hline
\end{tabular}




\begin{tabular}{lrrr}
$\mathrm{H}$ & -2.42307600 & -5.91680500 & -1.14906300 \\
$\mathrm{H}$ & -5.40968300 & 0.07981900 & 1.29798000 \\
$\mathrm{C}$ & -6.68264900 & -0.92833400 & 2.69803700 \\
$\mathrm{C}$ & -7.43401100 & 0.55944100 & 0.80846900 \\
$\mathrm{H}$ & -6.72165000 & -0.08503000 & 3.39541100 \\
$\mathrm{H}$ & -5.93274500 & -1.64309400 & 3.04916800 \\
$\mathrm{H}$ & -7.65454800 & -1.43335900 & 2.71752600 \\
$\mathrm{H}$ & -8.44213800 & 0.13711600 & 0.87278100 \\
$\mathrm{H}$ & -7.26169400 & 0.87990200 & -0.22418600 \\
$\mathrm{H}$ & -7.41420400 & 1.44645200 & 1.44870100 \\
$\mathrm{C}$ & -1.47851000 & 6.63363700 & -1.30301900 \\
$\mathrm{H}$ & -1.76716200 & 7.48428100 & -1.91724700 \\
$\mathrm{H}$ & -0.44017100 & 6.35742600 & -1.51696200 \\
$\mathrm{H}$ & -1.58747600 & 6.88607300 & -0.24317100 \\
$\mathrm{H}$ & 0.89821600 & 2.51514500 & 3.00910100 \\
$\mathrm{H}$ & 3.53047100 & 0.22045700 & 0.45350800 \\
\hline & & &
\end{tabular}

\section{Structure syn-(2S,3R)-TS Precomplex}

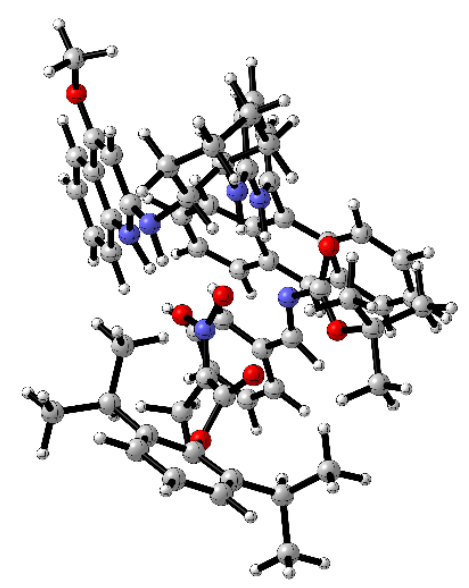

- Thermochemistry -

Zero-point correction $=$ 1.222407 (Hartree/Particle)

Thermal correction to Energy= 1.291877 Thermal correction to Enthalpy= 1.292821 
Thermal correction to Gibbs Free Energy=

1.115466

Sum of electronic and zero-point Energies $=$ $-3256.502118$

Sum of electronic and thermal Energies= $-3256.432648$

Sum of electronic and thermal Enthalpies= $-3256.431704$

Sum of electronic and thermal Free Energies= $-3256.609059$

Number of Imaginary Frequencies $=0$

$E$ (Single Point Energy) [SMD(Toluene)M06-2X/6-311++G(2d,2p)] = -3258.799320

\begin{tabular}{|c|c|c|c|}
\hline $\mathrm{O}$ & -3.85467900 & -0.55524700 & 0.31387800 \\
\hline $\mathrm{C}$ & -3.55250100 & -0.74582200 & -0.83729100 \\
\hline $\mathrm{O}$ & -4.48382900 & -0.91379700 & -1.82040900 \\
\hline $\mathrm{C}$ & -2.21092700 & -0.77024600 & -1.44905300 \\
\hline $\mathrm{C}$ & -1.95199600 & -1.34637900 & -2.80727800 \\
\hline $\mathrm{H}$ & -2.76987500 & -2.00533600 & -3.09543100 \\
\hline $\mathrm{H}$ & -1.01119400 & -1.90370400 & -2.80888200 \\
\hline $\mathrm{H}$ & -1.85937900 & -0.55414700 & -3.55951100 \\
\hline $\mathrm{N}$ & -1.25365800 & -0.05352400 & -0.89054400 \\
\hline $\mathrm{O}$ & -1.37089800 & 0.53756900 & 0.24449300 \\
\hline $\mathrm{O}$ & -0.14415000 & 0.10497800 & -1.53499200 \\
\hline $\mathrm{C}$ & 0.40027100 & -2.73693800 & -0.01283700 \\
\hline $\mathrm{C}$ & 1.19281600 & -2.94445200 & -1.22206000 \\
\hline $\mathrm{C}$ & 1.15177100 & -4.19995600 & -1.83989900 \\
\hline $\mathrm{C}$ & 1.98677900 & -1.92663800 & -1.76787100 \\
\hline $\mathrm{C}$ & 1.90426100 & -4.44351100 & -2.98367000 \\
\hline $\mathrm{C}$ & 2.72981400 & -2.17040100 & -2.91288900 \\
\hline $\mathrm{C}$ & 2.69376200 & -3.42748600 & -3.51870800 \\
\hline $\mathrm{C}$ & -0.29147300 & -1.58476200 & 1.88881700 \\
\hline $\mathrm{O}$ & 0.07823900 & -0.87126700 & 2.7987110 \\
\hline
\end{tabular}




\begin{tabular}{|c|c|c|c|}
\hline $\mathrm{O}$ & -1.39654800 & -2.30036700 & 1.84251100 \\
\hline $\mathrm{C}$ & -2.32379700 & -2.32429600 & 2.98507800 \\
\hline $\mathrm{C}$ & -2.78383300 & -0.91017300 & 3.32779400 \\
\hline $\mathrm{H}$ & -2.01582400 & -0.36799300 & 3.88110700 \\
\hline $\mathrm{H}$ & -3.68306100 & -0.97736800 & 3.94837500 \\
\hline $\mathrm{H}$ & -3.03121200 & -0.37513700 & 2.40748200 \\
\hline $\mathrm{C}$ & -1.63597700 & -3.01772000 & 4.15487100 \\
\hline $\mathrm{H}$ & -2.35230100 & -3.13857600 & 4.97296500 \\
\hline $\mathrm{H}$ & -0.79218300 & -2.42663000 & 4.51783700 \\
\hline $\mathrm{H}$ & -1.28463500 & -4.01117300 & 3.85689600 \\
\hline $\mathrm{C}$ & -3.47743000 & -3.15768000 & 2.44595600 \\
\hline $\mathrm{H}$ & -4.24778600 & -3.26727000 & 3.21441700 \\
\hline $\mathrm{H}$ & -3.12893400 & -4.15391300 & 2.15624700 \\
\hline $\mathrm{H}$ & -3.91573800 & -2.66106900 & 1.57654100 \\
\hline $\mathrm{N}$ & 0.51347500 & -1.69467900 & 0.72429800 \\
\hline $\mathrm{H}$ & -0.30740800 & -3.52724100 & 0.25522500 \\
\hline $\mathrm{H}$ & 3.28543900 & -3.61416600 & -4.40984700 \\
\hline $\mathrm{H}$ & 1.87267900 & -5.41861400 & -3.45835200 \\
\hline $\mathrm{H}$ & 3.36732100 & -1.38883500 & -3.31770400 \\
\hline $\mathrm{H}$ & 1.99354500 & -0.95058500 & -1.29488200 \\
\hline $\mathrm{H}$ & 0.53318200 & -4.98575800 & -1.41339000 \\
\hline $\mathrm{C}$ & -2.01903200 & 4.66270100 & 3.38328800 \\
\hline $\mathrm{C}$ & -0.85975000 & 4.20639800 & 4.27479900 \\
\hline $\mathrm{C}$ & -0.27360600 & 2.87349400 & 3.79823400 \\
\hline $\mathrm{C}$ & 0.15379300 & 2.98015200 & 2.33171900 \\
\hline $\mathrm{C}$ & -1.05310400 & 3.37916400 & 1.46482600 \\
\hline $\mathrm{C}$ & -1.60121500 & 4.73382100 & 1.90994000 \\
\hline $\mathrm{H}$ & -1.01380600 & 2.06816700 & 3.88497000 \\
\hline
\end{tabular}




\begin{tabular}{|c|c|c|c|}
\hline $\mathrm{H}$ & -1.19165200 & 4.11654000 & 5.31309400 \\
\hline $\mathrm{H}$ & -0.07076500 & 4.97189800 & 4.26246400 \\
\hline $\mathrm{H}$ & -2.39118000 & 5.63702700 & 3.71291800 \\
\hline $\mathrm{H}$ & -2.85232200 & 3.95512800 & 3.48192400 \\
\hline $\mathrm{H}$ & 0.90426400 & 3.77614200 & 2.24172000 \\
\hline $\mathrm{H}$ & -2.44690500 & 5.00207500 & 1.27017100 \\
\hline $\mathrm{H}$ & -0.83702900 & 5.51352800 & 1.78206600 \\
\hline $\mathrm{H}$ & 0.58715000 & 2.57998400 & 4.40996100 \\
\hline $\mathrm{N}$ & 0.74354400 & 1.75647400 & 1.80651500 \\
\hline $\mathrm{H}$ & 0.10583700 & 1.07478500 & 1.37117100 \\
\hline $\mathrm{N}$ & -0.77867700 & 3.25267700 & 0.02053400 \\
\hline $\mathrm{C}$ & 0.45759300 & 3.43136100 & -0.50624400 \\
\hline $\mathrm{C}$ & 1.27417700 & 4.55277800 & -0.20599000 \\
\hline $\mathrm{C}$ & 2.13486700 & 2.56419600 & -1.96594200 \\
\hline $\mathrm{C}$ & 2.52875900 & 4.64267300 & -0.76403700 \\
\hline $\mathrm{H}$ & 0.86560600 & 5.32833000 & 0.42560900 \\
\hline $\mathrm{C}$ & 2.52081100 & 1.54723700 & -2.85806400 \\
\hline $\mathrm{C}$ & 3.00319500 & 3.63021500 & -1.67386500 \\
\hline $\mathrm{O}$ & 3.38194400 & 5.63902200 & -0.54226100 \\
\hline $\mathrm{C}$ & 3.76832700 & 1.61413300 & -3.44178200 \\
\hline $\mathrm{H}$ & 1.82592700 & 0.73880500 & -3.07260500 \\
\hline $\mathrm{C}$ & 4.27205300 & 3.67570300 & -2.28482100 \\
\hline $\mathrm{C}$ & 4.64821000 & 2.67753800 & -3.15731700 \\
\hline $\mathrm{H}$ & 4.07414500 & 0.84003100 & -4.13896500 \\
\hline $\mathrm{H}$ & 4.93174100 & 4.50693300 & -2.06339300 \\
\hline $\mathrm{H}$ & 5.62002100 & 2.71637800 & -3.63796700 \\
\hline $\mathrm{N}$ & 0.90201100 & 2.49968800 & -1.35705700 \\
\hline $\mathrm{C}$ & 2.03320200 & 1.44170800 & 1.93289400 \\
\hline
\end{tabular}




\begin{tabular}{|c|c|c|c|}
\hline $\mathrm{C}$ & 3.00775000 & 2.26909300 & 2.55008600 \\
\hline $\mathrm{C}$ & 3.75554900 & -0.16457200 & 1.44088900 \\
\hline $\mathrm{C}$ & 4.32012600 & 1.86449900 & 2.56943100 \\
\hline $\mathrm{H}$ & 2.70641100 & 3.19461400 & 3.02127600 \\
\hline $\mathrm{C}$ & 4.71657000 & 0.63827200 & 2.00655900 \\
\hline $\mathrm{H}$ & 5.06131300 & 2.49686300 & 3.04820100 \\
\hline $\mathrm{H}$ & 5.74408000 & 0.29867700 & 2.03613000 \\
\hline $\mathrm{N}$ & 2.46095300 & 0.26299100 & 1.40734700 \\
\hline $\mathrm{C}$ & 4.03347400 & -1.50518300 & 0.86842600 \\
\hline $\mathrm{C}$ & 3.48243500 & -2.64280600 & 1.49187800 \\
\hline $\mathrm{C}$ & 4.82421500 & -1.61408500 & -0.29218100 \\
\hline $\mathrm{C}$ & 2.71395300 & -2.57538400 & 2.69908000 \\
\hline $\mathrm{C}$ & 3.69100500 & -3.93021700 & 0.89457300 \\
\hline $\mathrm{C}$ & 5.08131900 & -2.91957600 & -0.83268500 \\
\hline $\mathrm{C}$ & 5.33628800 & -0.48117700 & -1.00568600 \\
\hline $\mathrm{C}$ & 2.13692200 & -3.69472900 & 3.22338700 \\
\hline $\mathrm{H}$ & 2.56192000 & -1.61975300 & 3.18951300 \\
\hline $\mathrm{C}$ & 3.06107100 & -5.07764100 & 1.47126400 \\
\hline $\mathrm{C}$ & 4.49403500 & -4.03712600 & -0.24117600 \\
\hline $\mathrm{C}$ & 5.88853200 & -3.04630000 & -2.00642800 \\
\hline $\mathrm{H}$ & 5.11262300 & 0.52105100 & -0.65224100 \\
\hline $\mathrm{C}$ & 6.09311200 & -0.64593200 & -2.13006800 \\
\hline $\mathrm{C}$ & 2.29635300 & -4.96480700 & 2.59387800 \\
\hline $\mathrm{H}$ & 1.55054500 & -3.61937500 & 4.13383500 \\
\hline $\mathrm{H}$ & 3.21408400 & -6.04165900 & 0.99389300 \\
\hline $\mathrm{H}$ & 4.65804100 & -5.01727400 & -0.68383500 \\
\hline $\mathrm{C}$ & 6.38898400 & -1.94626600 & -2.63535900 \\
\hline $\mathrm{H}$ & 6.08261400 & -4.04460600 & -2.38915900 \\
\hline
\end{tabular}




\begin{tabular}{|c|c|c|c|}
\hline $\mathrm{H}$ & 6.47651800 & 0.22764000 & -2.64939700 \\
\hline $\mathrm{H}$ & 1.82289600 & -5.84019600 & 3.02652800 \\
\hline $\mathrm{H}$ & 7.00331200 & -2.05055900 & -3.52364200 \\
\hline $\mathrm{C}$ & -8.22448400 & 0.79629900 & -1.29360100 \\
\hline $\mathrm{C}$ & -7.19981300 & 1.51397500 & -1.90569200 \\
\hline $\mathrm{C}$ & -5.93257600 & 0.95552800 & -2.06252000 \\
\hline $\mathrm{C}$ & -5.74318100 & -0.34779900 & -1.58429900 \\
\hline $\mathrm{C}$ & -6.73465400 & -1.08804800 & -0.94798500 \\
\hline $\mathrm{C}$ & -7.99082900 & -0.48706100 & -0.81920200 \\
\hline $\mathrm{H}$ & -9.20756100 & 1.24343200 & -1.18564600 \\
\hline $\mathrm{H}$ & -7.39411600 & 2.51758200 & -2.27081800 \\
\hline $\mathrm{C}$ & -4.77502600 & 1.71880400 & -2.68543400 \\
\hline $\mathrm{C}$ & -6.47701900 & -2.47490500 & -0.39390200 \\
\hline $\mathrm{H}$ & -8.79395700 & -1.03599800 & -0.33463000 \\
\hline $\mathrm{C}$ & -5.22191700 & 2.71512300 & -3.75707400 \\
\hline $\mathrm{H}$ & -4.12109000 & 0.98128000 & -3.16527500 \\
\hline $\mathrm{C}$ & -3.95630500 & 2.44460000 & -1.60254800 \\
\hline $\mathrm{H}$ & -3.08812100 & 2.94199400 & -2.05192200 \\
\hline $\mathrm{H}$ & -3.60512900 & 1.76712400 & -0.81596100 \\
\hline $\mathrm{H}$ & -4.57339800 & 3.21218700 & -1.12132700 \\
\hline $\mathrm{H}$ & -5.77516800 & 3.55332200 & -3.32064200 \\
\hline $\mathrm{H}$ & -5.86051900 & 2.23759400 & -4.50550100 \\
\hline $\mathrm{H}$ & -4.34843800 & 3.13354100 & -4.26645200 \\
\hline $\mathrm{H}$ & -5.42365300 & -2.71799700 & -0.57034900 \\
\hline $\mathrm{C}$ & -7.33275700 & -3.52683700 & -1.10822800 \\
\hline $\mathrm{C}$ & -6.72174000 & -2.49758200 & 1.11920500 \\
\hline $\mathrm{H}$ & -7.11711000 & -4.52642100 & -0.71738600 \\
\hline $\mathrm{H}$ & -7.14207900 & -3.52742900 & -2.18514500 \\
\hline
\end{tabular}




$\begin{array}{lrrr}\mathrm{H} & -8.39958300 & -3.33127000 & -0.95524300 \\ \mathrm{H} & -7.77569200 & -2.30605500 & 1.34785900 \\ \mathrm{H} & -6.11817500 & -1.73329400 & 1.61808000 \\ \mathrm{H} & -6.46358000 & -3.47787700 & 1.53443400 \\ \mathrm{C} & 2.98224900 & 6.69537900 & 0.32244800 \\ \mathrm{H} & 3.82748400 & 7.37896300 & 0.36646000 \\ \mathrm{H} & 2.76243800 & 6.30835300 & 1.32323000 \\ \mathrm{H} & 2.10609200 & 7.21147200 & -0.08137300 \\ \mathrm{H} & -1.83716800 & 2.62925500 & 1.63664800 \\ \mathrm{H} & 1.74492900 & -0.40233800 & 1.01869800 \\ \mathrm{H} & -1.20606800 & 2.37892600 & -0.29655300 \\ \mathrm{H} & 0.37672000 & 1.56538000 & -1.43142000\end{array}$

Structure syn-(2R,3S)-TS Precomplex

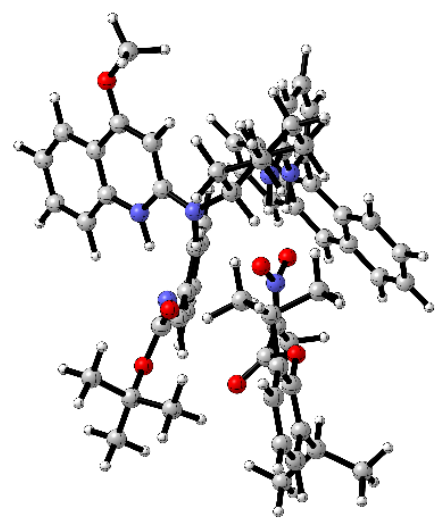

- Thermochemistry -

Zero-point correction=

Thermal correction to Energy=

Thermal correction to Enthalpy=

Thermal correction to Gibbs Free Energy=

Sum of electronic and zero-point Energies=

Sum of electronic and thermal Energies=
1.221508 (Hartree/Particle)

1.291090

1.292034

1.115626

$-3256.504101$

$-3256.434520$ 
Sum of electronic and thermal Enthalpies $=\quad-3256.433575$

Sum of electronic and thermal Free Energies $=\quad-3256.609983$

Number of Imaginary Frequencies $=0$

$E$ (Single Point Energy) $[S M D($ Toluene) M06-2X/6-311++G(2d,2p)] $=-3258.803935$

$\begin{array}{lrrr}\mathrm{O} & 3.21363200 & -1.64405900 & 1.37533700 \\ \mathrm{C} & 2.62862800 & -1.76966200 & 0.32306200 \\ \mathrm{O} & 3.22448800 & -1.68924000 & -0.88090100 \\ \mathrm{C} & 1.20176400 & -2.16135300 & 0.22226800 \\ \mathrm{C} & 0.71890100 & -3.46203400 & 0.77741400 \\ \mathrm{H} & 0.51370500 & -4.18221700 & -0.02346300 \\ \mathrm{H} & -0.21829100 & -3.31678400 & 1.32488800 \\ \mathrm{H} & 1.47613600 & -3.87862100 & 1.44663800 \\ \mathrm{~N} & 0.36717800 & -1.37016400 & -0.40589500 \\ \mathrm{O} & -0.84389300 & -1.77380900 & -0.56810300 \\ \mathrm{O} & 0.70402200 & -0.18683900 & -0.80088500 \\ \mathrm{C} & 0.79024700 & -0.35652600 & 2.58440900 \\ \mathrm{C} & -0.60206800 & -0.79860100 & 2.65056100 \\ \mathrm{C} & -1.56730400 & -0.23765700 & 1.80261300 \\ \mathrm{C} & -0.97005300 & -1.80321200 & 3.55297800 \\ \mathrm{C} & -2.88866900 & -0.64759800 & 1.89123700 \\ \mathrm{C} & -2.29664300 & -2.21270700 & 3.63583700 \\ \mathrm{C} & -3.25745300 & -1.63043000 & 2.80975900 \\ \mathrm{C} & 2.44049900 & 1.18351400 & 1.94930400 \\ \mathrm{O} & 2.76306900 & 1.88440500 & 1.00825900 \\ \mathrm{O} & 3.19082800 & 0.80120600 & 2.95898300 \\ \mathrm{C} & 4.60435800 & 1.20304800 & 3.07568900 \\ \mathrm{C} & 5.39985400 & 0.77571600 & 1.85036800 \\ \mathrm{H} & 6.46241300 & 0.95041800 & 2.04718200 \\ \mathrm{H} & 5.25252800 & -0.28807900 & 1.64338400 \\ \mathrm{C} & 5.05703000 & 0.43646600 & 4.31035300 \\ \mathrm{H} & 4.44975100 & 0.70778600 & 5.17850900 \\ \mathrm{H} & 6.10254900 & 0.67060300 & 4.52752700 \\ \mathrm{H} & 4.96695800 & -0.63976200 & 4.13708100 \\ \mathrm{C} & 4.65438100 & 2.70915700 & 3.30327300 \\ \mathrm{H} & 4.04848900 & 2.98592000 & 4.17142900 \\ \mathrm{H} & 4.29617200 & 3.24921500 & 2.42414200 \\ \mathrm{H} & 5.68825500 & 3.00947200 & 3.49676100 \\ \mathrm{~N} & 1.08979500 & 0.77925700 & 2.06658800 \\ \mathrm{H} & 1.56967200 & -1.01305600 & 2.98086800 \\ \mathrm{H} & -4.29395000 & -1.95232000 & 2.86674500\end{array}$




\begin{tabular}{|c|c|c|c|}
\hline $\mathrm{H}$ & -3.63712100 & -0.21700400 & 1.23309800 \\
\hline $\mathrm{H}$ & -2.58174300 & -2.98628100 & 4.34121400 \\
\hline $\mathrm{H}$ & -0.21412700 & -2.25380900 & 4.19133000 \\
\hline $\mathrm{H}$ & -1.25260700 & 0.46810400 & 1.03840600 \\
\hline $\mathrm{C}$ & 1.36635700 & 3.98231900 & -4.24555900 \\
\hline $\mathrm{C}$ & 0.12741000 & 3.48235800 & -4.99273400 \\
\hline $\mathrm{C}$ & -0.39810600 & 2.18077100 & -4.38303600 \\
\hline $\mathrm{C}$ & -0.71061500 & 2.35713900 & -2.88961400 \\
\hline $\mathrm{C}$ & 0.57396800 & 2.81266200 & -2.16625800 \\
\hline $\mathrm{C}$ & 1.08026600 & 4.13875100 & -2.74928900 \\
\hline $\mathrm{H}$ & 0.34962000 & 1.38328200 & -4.48276500 \\
\hline $\mathrm{H}$ & 0.35492200 & 3.33171100 & -6.05190900 \\
\hline $\mathrm{H}$ & -0.65912600 & 4.24904700 & -4.94570300 \\
\hline $\mathrm{H}$ & 1.70590100 & 4.93573300 & -4.66077900 \\
\hline $\mathrm{H}$ & 2.18838200 & 3.26664400 & -4.38202900 \\
\hline $\mathrm{H}$ & -1.47989800 & 3.13571000 & -2.78378200 \\
\hline $\mathrm{H}$ & 1.97546400 & 4.44801500 & -2.20202000 \\
\hline $\mathrm{H}$ & 0.33454000 & 4.93165700 & -2.60704500 \\
\hline $\mathrm{H}$ & -1.29634600 & 1.83776500 & -4.90856300 \\
\hline $\mathrm{N}$ & -1.20064200 & 1.14437800 & -2.26321300 \\
\hline $\mathrm{H}$ & -0.50809500 & 0.56250000 & -1.73840500 \\
\hline $\mathrm{N}$ & 0.47289600 & 2.78590200 & -0.70894800 \\
\hline $\mathrm{H}$ & 1.12088400 & 2.14759200 & -0.23518000 \\
\hline $\mathrm{C}$ & -0.46916600 & 3.34857000 & 0.05226800 \\
\hline $\mathrm{C}$ & -1.37762300 & 4.35175200 & -0.39406800 \\
\hline $\mathrm{C}$ & -1.47513800 & 3.40291800 & 2.23230200 \\
\hline $\mathrm{C}$ & -2.30911200 & 4.86071700 & 0.47714400 \\
\hline $\mathrm{H}$ & -1.28517400 & 4.72744400 & -1.39934700 \\
\hline $\mathrm{C}$ & -1.50908200 & 2.87940400 & 3.53455100 \\
\hline $\mathrm{C}$ & -2.39329200 & 4.38869700 & 1.83702700 \\
\hline $\mathrm{O}$ & -3.18998200 & 5.80792500 & 0.16171400 \\
\hline $\mathrm{C}$ & -2.45110300 & 3.35600000 & 4.42256700 \\
\hline $\mathrm{H}$ & -0.80015200 & 2.10708500 & 3.81763800 \\
\hline $\mathrm{C}$ & -3.34142300 & 4.86463800 & 2.76177700 \\
\hline $\mathrm{C}$ & -3.36772100 & 4.35319700 & 4.04160100 \\
\hline $\mathrm{H}$ & -2.48305100 & 2.95435900 & 5.42997200 \\
\hline $\mathrm{H}$ & -4.04053400 & 5.63143900 & 2.44836200 \\
\hline $\mathrm{H}$ & -4.09629700 & 4.71741500 & 4.75721300 \\
\hline $\mathrm{N}$ & -0.54665700 & 2.93249000 & 1.32847700 \\
\hline $\mathrm{C}$ & -2.44678700 & 0.68121700 & -2.40615800 \\
\hline $\mathrm{C}$ & -3.48119900 & 1.30283800 & -3.14794800 \\
\hline $\mathrm{C}$ & -3.94004700 & -1.10811700 & -1.83079200 \\
\hline $\mathrm{C}$ & -4.71222200 & 0.68995100 & -3.20917500 \\
\hline $\mathrm{H}$ & -3.28929300 & 2.22866200 & -3.67446000 \\
\hline $\mathrm{C}$ & -4.95959300 & -0.52996400 & -2.55698200 \\
\hline $\mathrm{H}$ & -5.50427200 & 1.15253000 & -3.79010600 \\
\hline
\end{tabular}




\begin{tabular}{|c|c|c|c|}
\hline $\mathrm{H}$ & -5.91769400 & -1.02959900 & -2.62590700 \\
\hline $\mathrm{N}$ & -2.73619800 & -0.48075200 & -1.76844100 \\
\hline $\mathrm{C}$ & -4.05235100 & -2.39854700 & -1.10463900 \\
\hline $\mathrm{C}$ & -3.21008100 & -3.46976500 & -1.47288800 \\
\hline $\mathrm{C}$ & -4.99226700 & -2.53212600 & -0.05996700 \\
\hline $\mathrm{C}$ & -2.30892500 & -3.41601100 & -2.58527200 \\
\hline $\mathrm{C}$ & -3.27813100 & -4.69482700 & -0.72856200 \\
\hline $\mathrm{C}$ & -5.06277700 & -3.77678600 & 0.65290300 \\
\hline $\mathrm{C}$ & -5.86616600 & -1.47696400 & 0.36411100 \\
\hline $\mathrm{C}$ & -1.51788100 & -4.47982800 & -2.90150400 \\
\hline $\mathrm{H}$ & -2.26171000 & -2.51918500 & -3.19351600 \\
\hline $\mathrm{C}$ & -2.41748600 & -5.78208500 & -1.08072300 \\
\hline $\mathrm{C}$ & -4.19718900 & -4.81535500 & 0.31193100 \\
\hline $\mathrm{C}$ & -6.01172300 & -3.93016800 & 1.71283300 \\
\hline $\mathrm{H}$ & -5.81859300 & -0.51393000 & -0.13322900 \\
\hline $\mathrm{C}$ & -6.75309600 & -1.65956100 & 1.38583900 \\
\hline $\mathrm{C}$ & -1.55881800 & -5.67975300 & -2.13251900 \\
\hline $\mathrm{H}$ & -0.84748100 & -4.41712700 & -3.75257600 \\
\hline $\mathrm{H}$ & -2.47964300 & -6.69578200 & -0.49626900 \\
\hline $\mathrm{H}$ & -4.24946500 & -5.75014000 & 0.86597800 \\
\hline $\mathrm{C}$ & -6.83917800 & -2.90792900 & 2.06934700 \\
\hline $\mathrm{H}$ & -6.05494300 & -4.88685200 & 2.22623300 \\
\hline $\mathrm{H}$ & -7.40431900 & -0.84399300 & 1.68422400 \\
\hline $\mathrm{H}$ & -0.91561400 & -6.51122700 & -2.40141900 \\
\hline $\mathrm{H}$ & -7.55930800 & -3.03370000 & 2.87098900 \\
\hline $\mathrm{C}$ & 7.26002700 & -0.73749000 & -1.02243200 \\
\hline $\mathrm{C}$ & 6.87017900 & -1.97625500 & -0.53170100 \\
\hline $\mathrm{C}$ & 5.52100200 & -2.33884600 & -0.46873700 \\
\hline $\mathrm{C}$ & 4.59541400 & -1.39044100 & -0.90362800 \\
\hline $\mathrm{C}$ & 4.94639100 & -0.14199400 & -1.42390700 \\
\hline $\mathrm{C}$ & 6.30480400 & 0.16744100 & -1.47813500 \\
\hline $\mathrm{H}$ & 8.31307200 & -0.47729500 & -1.06155700 \\
\hline $\mathrm{H}$ & 7.62386100 & -2.68185900 & -0.19262800 \\
\hline $\mathrm{C}$ & 5.12185300 & -3.71322200 & 0.03773400 \\
\hline $\mathrm{C}$ & 3.86205300 & 0.78491700 & -1.94221900 \\
\hline $\mathrm{H}$ & 6.62449600 & 1.12866900 & -1.86672500 \\
\hline $\mathrm{C}$ & 5.74112000 & -4.81451300 & -0.83201200 \\
\hline $\mathrm{H}$ & 4.03396600 & -3.81028900 & -0.04340600 \\
\hline $\mathrm{C}$ & 5.51016700 & -3.89446800 & 1.50941700 \\
\hline $\mathrm{H}$ & 5.18134500 & -4.87375600 & 1.87227400 \\
\hline $\mathrm{H}$ & 5.05497900 & -3.11866900 & 2.13029000 \\
\hline $\mathrm{H}$ & 6.59757600 & -3.84283500 & 1.63124700 \\
\hline $\mathrm{H}$ & 5.40426400 & -5.79925900 & -0.49320100 \\
\hline $\mathrm{H}$ & 6.83414600 & -4.79775600 & -0.76989600 \\
\hline $\mathrm{H}$ & 5.46127600 & -4.69488900 & -1.88245000 \\
\hline $\mathrm{H}$ & 3.01698400 & 0.72411700 & -1.24452600 \\
\hline
\end{tabular}




$\begin{array}{lrrr}\mathrm{C} & 3.37995600 & 0.27937100 & -3.31028400 \\ \mathrm{C} & 4.31436800 & 2.24079400 & -2.02611500 \\ \mathrm{H} & 2.62261800 & 0.95136100 & -3.73341000 \\ \mathrm{H} & 2.94026100 & -0.71750400 & -3.22426600 \\ \mathrm{H} & 4.21528500 & 0.23579400 & -4.01760500 \\ \mathrm{H} & 5.09834000 & 2.37922800 & -2.77861300 \\ \mathrm{H} & 4.68398000 & 2.60456800 & -1.06241800 \\ \mathrm{H} & 3.47367100 & 2.87654300 & -2.32250700 \\ \mathrm{C} & -3.17162400 & 6.34425200 & -1.15393000 \\ \mathrm{H} & -3.96782400 & 7.08519200 & -1.18381400 \\ \mathrm{H} & -3.36655700 & 5.55811100 & -1.89139700 \\ \mathrm{H} & -2.20948200 & 6.82344400 & -1.36070900 \\ \mathrm{H} & 1.33037500 & 2.04552800 & -2.37640500 \\ \mathrm{H} & -1.92588800 & -0.95306500 & -1.22308500 \\ \mathrm{H} & 5.11330900 & 1.34824800 & 0.96781000 \\ \mathrm{H} & 0.06322100 & 2.12858500 & 1.62032400\end{array}$

\section{Structure anti-(2S,3S)-TS Precomplex}

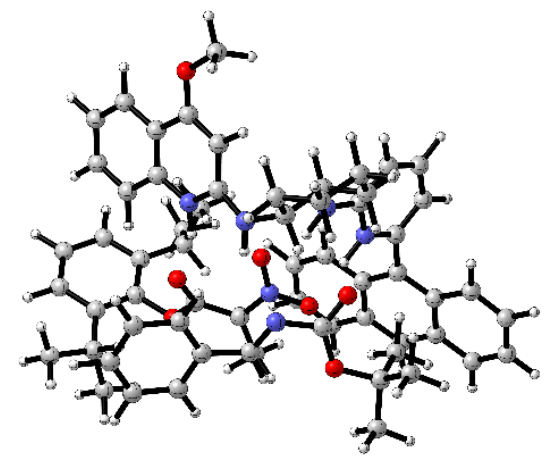

- Thermochemistry -

Zero-point correction $=$

Thermal correction to Energy=

Thermal correction to Enthalpy=

Thermal correction to Gibbs Free Energy=

Sum of electronic and zero-point Energies=

Sum of electronic and thermal Energies=

Sum of electronic and thermal Enthalpies=

Sum of electronic and thermal Free Energies=
1.224850 (Hartree/Particle)

1.293946

1.294890

1.121130

$-3256.519092$

$-3256.449995$

$-3256.449051$

$-3256.622811$ 
Number of Imaginary Frequencies $=0$

E (Single Point Energy) [SMD(Toluene)M06-2X/6-311++G(2d,2p)] = -3258.813529

$\begin{array}{lrrr}\mathrm{O} & -2.48665900 & -0.80010900 & 0.50555300 \\ \mathrm{C} & -1.79283800 & -1.79952500 & 0.40762200 \\ \mathrm{O} & -2.36236800 & -3.01218900 & 0.17095400 \\ \mathrm{C} & -0.34894700 & -1.90116300 & 0.51252000 \\ \mathrm{C} & 0.38176700 & -3.17573400 & 0.79865200 \\ \mathrm{H} & 0.98955200 & -3.07857800 & 1.70548100 \\ \mathrm{H} & 1.06730500 & -3.42364300 & -0.01843000 \\ \mathrm{H} & -0.32824200 & -3.99190100 & 0.93111900 \\ \mathrm{~N} & 0.38357100 & -0.81053900 & 0.26255500 \\ \mathrm{O} & -0.11667600 & 0.29995700 & -0.10033000 \\ \mathrm{O} & 1.64986300 & -0.89187000 & 0.37227500 \\ \mathrm{C} & 0.09577600 & -0.26889700 & 3.19634000 \\ \mathrm{C} & -1.31401100 & -0.34212700 & 3.57773200 \\ \mathrm{C} & -1.78760600 & -1.54173600 & 4.12006800 \\ \mathrm{C} & -2.17111300 & 0.76160400 & 3.47937100 \\ \mathrm{C} & -3.09633200 & -1.63363300 & 4.58010300 \\ \mathrm{C} & -3.48465200 & 0.65877300 & 3.91402100 \\ \mathrm{C} & -3.94615500 & -0.53502500 & 4.47178200 \\ \mathrm{C} & 2.04525900 & 0.76832600 & 2.51995800 \\ \mathrm{O} & 2.57644000 & 1.60856600 & 1.82286700 \\ \mathrm{O} & 2.66853500 & -0.18880600 & 3.19283100 \\ \mathrm{C} & 4.11313300 & -0.40220800 & 3.07154800 \\ \mathrm{C} & 4.85487800 & 0.81172300 & 3.61996400 \\ \mathrm{H} & 4.69307800 & 1.68951500 & 2.99295400 \\ \mathrm{H} & 5.92652500 & 0.59314500 & 3.64910500 \\ \mathrm{H} & 4.52215000 & 1.02998400 & 4.63922100 \\ \mathrm{C} & 4.48149600 & -0.70846700 & 1.62564300 \\ \mathrm{H} & 3.85762900 & -1.51756700 & 1.23265700 \\ \mathrm{H} & 5.53104400 & -1.01907600 & 1.58031200 \\ \mathrm{H} & 4.35707500 & 0.17336800 & 0.99246100 \\ \mathrm{C} & 4.34157200 & -1.62235300 & 3.95481400 \\ \mathrm{H} & 3.77620400 & -2.47825400 & 3.57461200 \\ \mathrm{H} & 4.02428700 & -1.41827400 & 4.98123800 \\ \mathrm{H} & 5.40354900 & -1.88183900 & 3.96168900 \\ \mathrm{~N} & 0.64477600 & 0.80053000 & 2.74837700 \\ \mathrm{H} & 0.68441400 & -1.17832400 & 3.35367100 \\ \mathrm{H} & -4.96989000 & -0.60615000 & 4.82647900 \\ \mathrm{H} & -3.45413500 & -2.56069900 & 5.01787600\end{array}$




$\begin{array}{lrrr}\mathrm{H} & -4.14948300 & 1.51312100 & 3.83879600 \\ \mathrm{H} & -1.78873400 & 1.70016000 & 3.08923000 \\ \mathrm{H} & -1.11884400 & -2.39614000 & 4.19543100 \\ \mathrm{C} & 1.04913500 & 5.83134800 & 2.19635800 \\ \mathrm{C} & 2.02834800 & 6.01198200 & 1.03514400 \\ \mathrm{C} & 2.41184400 & 4.66550300 & 0.41527200 \\ \mathrm{C} & 1.16548800 & 3.92051800 & -0.08700100 \\ \mathrm{C} & 0.20976400 & 3.73809200 & 1.11427500 \\ \mathrm{C} & -0.20127400 & 5.07466500 & 1.73918500 \\ \mathrm{H} & 2.90086400 & 4.02311300 & 1.15770300 \\ \mathrm{H} & 2.93040600 & 6.52938500 & 1.37461300 \\ \mathrm{H} & 1.56936000 & 6.65153700 & 0.26772900 \\ \mathrm{H} & 0.76526500 & 6.80161300 & 2.61453900 \\ \mathrm{H} & 1.53941600 & 5.26665800 & 2.99942900 \\ \mathrm{H} & 0.67566900 & 4.51415000 & -0.87573300 \\ \mathrm{H} & 0.79745400 & 3.20093000 & 1.86724100 \\ \mathrm{H} & -0.87126300 & 4.87300400 & 2.58079200 \\ \mathrm{H} & -0.75528100 & 5.70361100 & 1.03240600 \\ \mathrm{H} & 3.12616100 & 4.81858200 & -0.39915200 \\ \mathrm{~N} & 1.44914400 & 2.58074800 & -0.59095900 \\ \mathrm{H} & 1.03878000 & 1.80200500 & -0.06925100 \\ \mathrm{~N} & -0.88891900 & 2.80790600 & 0.84799900 \\ \mathrm{H} & -0.70572400 & 1.86170200 & 1.19314500 \\ \mathrm{C} & -1.86065800 & 2.91624800 & -0.07083900 \\ \mathrm{C} & -2.13908600 & 4.11496900 & -0.78256100 \\ \mathrm{C} & -3.73259500 & 1.84152500 & -1.12844900 \\ \mathrm{C} & -2.35000200 & 0.92694700 & 0.12047000 \\ \mathrm{H} & -3.20246200 & 4.15909300 & -1.64968800 \\ \mathrm{C} & -1.50923000 & 4.97281000 & -0.61967700 \\ \mathrm{H} & -4.53522800 & 0.69445400 & -1.25863500 \\ \mathrm{C} & -4.05276900 & 3.01275300 & -1.83746000 \\ \mathrm{C} & -3.54146700 & 5.23137000 & -2.36235100 \\ \mathrm{H} & -5.63056300 & 0.73945200 & -2.09754800 \\ \mathrm{C} & -4.28856000 & -0.20417000 & -0.70091200 \\ \mathrm{H} & -5.17420100 & 3.03121800 & -2.68850400 \\ \mathrm{C} & -5.95453400 & 1.90356700 & -2.81759600 \\ \mathrm{C} & -6.24721600 & -0.14854100 & -2.19378700 \\ \mathrm{H} & -5.40647400 & 3.94224700 & -3.22771600 \\ \mathrm{H} & -6.82113300 & 1.91394900 & -3.46946000 \\ \mathrm{H} & 2.62630300 & 1.83398700 & -0.29443600 \\ \mathrm{H} & 2.06119600 & 3.06318700 & -2.36429800 \\ \mathrm{H} & 0.40127300 & -2.11789900 \\ \mathrm{H} & 0.30000200 & -0.62303200 \\ \mathrm{H} & 2.596000 & 2.54244300 & -3.17303500 \\ \mathrm{H} & 4.08577400 & -2.46805500\end{array}$




\begin{tabular}{|c|c|c|c|}
\hline & 4.44676000 & 1.19963700 & -3.05815800 \\
\hline & 4.50474800 & 3.17652000 & -3.92303100 \\
\hline & 5.22217400 & 0.78155600 & -3.68758000 \\
\hline & 2.85937300 & 0.93223800 & -1.33247500 \\
\hline & 4.20431700 & -1.01474600 & -1.86841400 \\
\hline & 5.50120100 & -1.31035400 & -1.39724000 \\
\hline & 3.25842500 & -2.03228800 & -2.10233200 \\
\hline & 6.48718500 & -0.30997700 & -1.10997700 \\
\hline & 5.84577300 & -2.68018200 & -1.13757900 \\
\hline & 3.62978200 & -3.39662100 & -1.85604100 \\
\hline & 1.94753300 & -1.77869900 & -2.62160500 \\
\hline & 7.71618200 & -0.65131200 & -0.62600600 \\
\hline & 6.24502700 & 0.73617400 & -1.26084300 \\
\hline & 7.14635300 & -2.99477400 & -0.63159600 \\
\hline & 4.90626300 & -3.68303100 & -1.37392200 \\
\hline & 2.69212100 & -4.44293100 & -2.13005200 \\
\hline 1 & 1.63506700 & -0.75910800 & -2.82676800 \\
\hline$C$ & 1.09030100 & -2.80570700 & -2.88517900 \\
\hline - & 8.05860300 & -2.01399000 & -0.38499600 \\
\hline $\mathrm{H}$ & 8.44391200 & 0.12518300 & -0.41244700 \\
\hline $\mathrm{H}$ & 7.38598700 & -4.03842300 & -0.44796200 \\
\hline & 5.17976100 & -4.71865900 & -1.18383500 \\
\hline $\mathrm{C}$ & 1.45921700 & -4.16034600 & -2.63514300 \\
\hline $\mathrm{H}$ & 2.99579200 & -5.46796900 & -1.93640900 \\
\hline $\mathrm{H}$ & 0.11523800 & -2.59246600 & -3.30718000 \\
\hline H & 9.04335500 & -2.26062900 & -0.00236500 \\
\hline $\mathrm{H}$ & 0.75338700 & -4.95598300 & -2.85272200 \\
\hline $\mathrm{C}$ & -6.14694300 & -2.96691600 & -1.52737300 \\
\hline $\mathrm{C}$ & -5.02082900 & -2.84938500 & -2.33283100 \\
\hline $\mathrm{C}$ & -3.73769000 & -2.85493700 & -1.77729000 \\
\hline $\mathrm{C}$ & -3.64715200 & -2.97927100 & -0.38960500 \\
\hline $\mathrm{C}$ & -4.75447100 & -3.09514200 & 0.45496700 \\
\hline & -6.01345400 & -3.08804800 & -0.14622200 \\
\hline $\mathrm{H}$ & -7.13593100 & -2.97059700 & -1.97595800 \\
\hline $\mathrm{H}$ & -5.13672400 & -2.75743600 & -3.40887700 \\
\hline $\mathrm{C}$ & -2.49696200 & -2.70349300 & -2.64036100 \\
\hline $\mathrm{C}$ & -4.54496000 & -3.22704100 & 1.95036400 \\
\hline $\mathrm{H}$ & -6.90293900 & -3.17320600 & 0.46856300 \\
\hline $\mathrm{C}$ & -2.59891000 & -3.50070400 & -3.94474700 \\
\hline $\mathrm{H}$ & -1.65216700 & -3.10342800 & -2.06684300 \\
\hline $\mathrm{C}$ & -2.21928800 & -1.22024700 & -2.93798800 \\
\hline $\mathrm{H}$ & -3.10074300 & -0.75626200 & -3.39515600 \\
\hline $\mathrm{H}$ & -1.38689100 & -1.11332700 & -3.64290400 \\
\hline $\mathrm{H}$ & -1.96777900 & -0.65677800 & -2.03271200 \\
\hline $\mathrm{H}$ & -1.63685800 & -3.49696700 & -4.46870500 \\
\hline & -3.33442800 & -3.06256000 & -4.62720400 \\
\hline
\end{tabular}




$\begin{array}{lrrr}\mathrm{H} & -2.88346300 & -4.53984600 & -3.75739100 \\ \mathrm{H} & -3.79814400 & -2.47341200 & 2.22778100 \\ \mathrm{C} & -3.98200500 & -4.61172500 & 2.30063500 \\ \mathrm{C} & -5.80944800 & -2.95042000 & 2.76089700 \\ \mathrm{H} & -3.77442700 & -4.67822700 & 3.37444300 \\ \mathrm{H} & -3.05593000 & -4.81699000 & 1.75758900 \\ \mathrm{H} & -4.70794100 & -5.39211500 & 2.04870200 \\ \mathrm{H} & -6.57682900 & -3.71009600 & 2.57683100 \\ \mathrm{H} & -6.23289200 & -1.96757100 & 2.52979800 \\ \mathrm{H} & -5.57985100 & -2.98050600 & 3.83053400 \\ \mathrm{C} & -2.76830100 & 6.41505500 & -2.22204200 \\ \mathrm{H} & -1.73108000 & 6.23482400 & -2.52310800 \\ \mathrm{H} & -2.80265900 & 6.77676900 & -1.18924900 \\ \mathrm{H} & -3.22372900 & 7.14726700 & -2.88563800\end{array}$

Structure anti-(2R,3R)-TS2 Precomplex

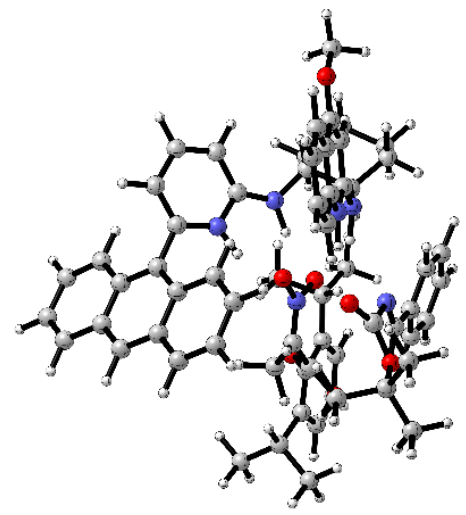

- Thermochemistry -

Zero-point correction $=$

Thermal correction to Energy=

Thermal correction to Enthalpy=

Thermal correction to Gibbs Free Energy=

Sum of electronic and zero-point Energies=

Sum of electronic and thermal Energies=

Sum of electronic and thermal Enthalpies=

Sum of electronic and thermal Free Energies=
1.223350 (Hartree/Particle)

1.292838

1.293782

1.116495

$-3256.500522$

$-3256.431034$

$-3256.430090$

$-3256.607377$ 
Number of Imaginary Frequencies $=0$

E (Single Point Energy) [SMD(Toluene)M06-2X/6-311++G(2d,2p)] = -3258.796537

$\begin{array}{llll}\mathrm{O} & -3.58537100 & 0.16054700 & 1.91607200 \\ \mathrm{C} & -2.95357900 & 0.44225100 & 0.92344200 \\ \mathrm{O} & -3.46267300 & 0.51017200 & -0.31498500 \\ \mathrm{C} & -1.52090100 & 0.83838100 & 0.97321600 \\ \mathrm{C} & -1.03896300 & 1.90393000 & 1.90252000 \\ \mathrm{H} & -0.83044900 & 2.83922200 & 1.36690400 \\ \mathrm{H} & -0.10301800 & 1.59739600 & 2.37886500 \\ \mathrm{H} & -1.80329000 & 2.09702800 & 2.65886200 \\ \mathrm{~N} & -0.66494800 & 0.17298800 & 0.24474700 \\ \mathrm{O} & 0.59978600 & 0.46768500 & 0.28548200 \\ \mathrm{O} & -1.00089800 & -0.83261800 & -0.49393700 \\ \mathrm{C} & -1.41907100 & -2.03575900 & 2.35054000 \\ \mathrm{C} & -2.04824500 & -3.02104700 & 1.47377600 \\ \mathrm{C} & -3.43160800 & -2.96315500 & 1.26854100 \\ \mathrm{C} & -1.29201800 & -4.03769900 & 0.87328200 \\ \mathrm{C} & -4.05292800 & -3.90561100 & 0.45438800 \\ \mathrm{C} & -1.91807900 & -4.98153000 & 0.07175600 \\ \mathrm{C} & -3.29839100 & -4.91400200 & -0.13918400 \\ \mathrm{C} & 0.36329800 & -0.97529500 & 3.34569600 \\ \mathrm{O} & 1.42594600 & -0.40614800 & 3.14949300 \\ \mathrm{O} & -0.37246700 & -0.85286000 & 4.43853400 \\ \mathrm{C} & 0.09008200 & -0.09032400 & 5.60683900 \\ \mathrm{C} & 0.27396000 & 1.38025500 & 5.25123300\end{array}$




\begin{tabular}{|c|c|c|c|}
\hline $\mathrm{H}$ & 1.08109400 & 1.52049400 & 4.52986600 \\
\hline $\mathrm{H}$ & 0.51504000 & 1.93458000 & 6.16360800 \\
\hline $\mathrm{H}$ & -0.65121200 & 1.79110000 & 4.83763300 \\
\hline $\mathrm{C}$ & 1.36853500 & -0.72402400 & 6.14451000 \\
\hline $\mathrm{H}$ & 1.21377600 & -1.79178300 & 6.32495400 \\
\hline $\mathrm{H}$ & 1.62839400 & -0.25105400 & 7.09593200 \\
\hline $\mathrm{H}$ & 2.20295800 & -0.59480700 & 5.45309800 \\
\hline $\mathrm{C}$ & -1.05841400 & -0.26772100 & 6.59139700 \\
\hline $\mathrm{H}$ & -1.20783600 & -1.32649600 & 6.81888100 \\
\hline $\mathrm{H}$ & -1.98496300 & 0.13498900 & 6.17317900 \\
\hline $\mathrm{H}$ & -0.83435200 & 0.26222700 & 7.52100500 \\
\hline $\mathrm{N}$ & -0.14245200 & -1.88776500 & 2.40823900 \\
\hline $\mathrm{H}$ & -2.09054400 & -1.42007000 & 2.95600800 \\
\hline $\mathrm{H}$ & -3.78459600 & -5.65776300 & -0.76358500 \\
\hline $\mathrm{H}$ & -5.12297700 & -3.84550600 & 0.28113800 \\
\hline $\mathrm{H}$ & -1.34383900 & -5.78937700 & -0.37344200 \\
\hline $\mathrm{H}$ & -0.22414600 & -4.08425500 & 1.07157500 \\
\hline $\mathrm{H}$ & -4.01207100 & -2.16796100 & 1.72866000 \\
\hline $\mathrm{C}$ & 0.37976800 & -5.00060300 & -3.01541700 \\
\hline $\mathrm{C}$ & 0.73625500 & -4.18238900 & -4.25964800 \\
\hline $\mathrm{C}$ & 0.46630100 & -2.69286700 & -4.03341100 \\
\hline $\mathrm{C}$ & 1.23700200 & -2.17027900 & -2.81713100 \\
\hline $\mathrm{C}$ & 0.88157300 & -3.01728700 & -1.57374700 \\
\hline C & 1.16091800 & -4.50690300 & -1.79434900 \\
\hline $\mathrm{H}$ & -0.60446300 & -2.53376400 & -3.85399300 \\
\hline $\mathrm{H}$ & 0.16678800 & -4.53440900 & -5.12458000 \\
\hline $\mathrm{H}$ & 1.79867700 & -4.33196500 & -4.50008800 \\
\hline $\mathrm{H}$ & 0.58261100 & -6.06253900 & -3.18367600 \\
\hline
\end{tabular}




\begin{tabular}{|c|c|c|c|}
\hline $\mathrm{H}$ & -0.69629700 & -4.90674300 & -2.81323400 \\
\hline $\mathrm{H}$ & 2.31311200 & -2.24584000 & -3.02102400 \\
\hline $\mathrm{H}$ & -0.20344600 & -2.91720900 & -1.43637300 \\
\hline $\mathrm{H}$ & 0.87494000 & -5.05464200 & -0.89068700 \\
\hline $\mathrm{H}$ & 2.22911700 & -4.69949800 & -1.95213400 \\
\hline $\mathrm{H}$ & 0.73201000 & -2.10050600 & -4.91609600 \\
\hline $\mathrm{N}$ & 0.92862900 & -0.78644300 & -2.49248900 \\
\hline $\mathrm{H}$ & 0.18374700 & -0.67647300 & -1.77428700 \\
\hline $\mathrm{N}$ & 1.42163500 & -2.45621000 & -0.33385400 \\
\hline $\mathrm{H}$ & 0.72531600 & -1.94907900 & 0.22152800 \\
\hline $\mathrm{C}$ & 2.69931900 & -2.17232600 & -0.06764600 \\
\hline $\mathrm{C}$ & 3.79079800 & -2.65348000 & -0.84915100 \\
\hline $\mathrm{C}$ & 4.25133800 & -1.11292100 & 1.43080500 \\
\hline $\mathrm{H}$ & 2.18724700 & -1.01591900 & 1.56580300 \\
\hline $\mathrm{C}$ & 5.07825500 & -2.33935700 & -0.49840700 \\
\hline $\mathrm{H}$ & 3.58186400 & -3.28248100 & -1.69715500 \\
\hline $\mathrm{C}$ & 4.46015800 & -0.38387200 & 2.61396700 \\
\hline $\mathrm{C}$ & 5.35189800 & -1.54965900 & 0.67412100 \\
\hline $\mathrm{O}$ & 6.15629500 & -2.73257300 & -1.17413900 \\
\hline $\mathrm{C}$ & 5.75072700 & -0.08340400 & 3.00089800 \\
\hline $\mathrm{H}$ & 3.60436100 & -0.09032100 & 3.21025800 \\
\hline $\mathrm{C}$ & 6.65876900 & -1.23234000 & 1.08908200 \\
\hline $\mathrm{C}$ & 6.85635800 & -0.49948000 & 2.23886900 \\
\hline $\mathrm{H}$ & 5.91124500 & 0.47789900 & 3.91573300 \\
\hline $\mathrm{H}$ & 7.49456600 & -1.57914300 & 0.49243600 \\
\hline $\mathrm{H}$ & 7.86141100 & -0.25291200 & 2.56222800 \\
\hline $\mathrm{N}$ & 2.96623100 & -1.40898100 & 1.00920900 \\
\hline C & 1.71378400 & 0.26009500 & -2.75743300 \\
\hline
\end{tabular}




\begin{tabular}{|c|c|c|c|}
\hline $\mathrm{C}$ & 2.71918900 & 0.30141000 & -3.75407700 \\
\hline $\mathrm{C}$ & 2.12811400 & 2.57788800 & -2.25301200 \\
\hline $\mathrm{H}$ & 0.95525000 & 1.25069300 & -1.13536900 \\
\hline $\mathrm{C}$ & 3.39894200 & 1.47830900 & -3.96143100 \\
\hline $\mathrm{H}$ & 2.90443200 & -0.57456700 & -4.36319100 \\
\hline $\mathrm{C}$ & 3.09899000 & 2.64084300 & -3.22588300 \\
\hline $\mathrm{H}$ & 4.16570200 & 1.51912900 & -4.72894300 \\
\hline $\mathrm{H}$ & 3.60323200 & 3.57999400 & -3.41554600 \\
\hline $\mathrm{N}$ & 1.50508600 & 1.38772000 & -2.02719200 \\
\hline $\mathrm{C}$ & 1.66289100 & 3.74216200 & -1.45614700 \\
\hline $\mathrm{C}$ & 1.03976800 & 4.81122900 & -2.13804200 \\
\hline $\mathrm{C}$ & 1.81030300 & 3.75211500 & -0.05307700 \\
\hline $\mathrm{C}$ & 0.81232700 & 4.82746900 & -3.55365500 \\
\hline $\mathrm{C}$ & 0.56869800 & 5.93582400 & -1.37808800 \\
\hline $\mathrm{C}$ & 1.30383400 & 4.87389600 & 0.68838100 \\
\hline $\mathrm{C}$ & 2.46438600 & 2.70983400 & 0.68012800 \\
\hline $\mathrm{C}$ & 0.20767000 & 5.89052600 & -4.15799400 \\
\hline $\mathrm{H}$ & 1.11320600 & 3.97627900 & -4.15360800 \\
\hline $\mathrm{C}$ & -0.05315700 & 7.03343500 & -2.05252200 \\
\hline $\mathrm{C}$ & 0.70691400 & 5.93451000 & 0.00854200 \\
\hline $\mathrm{C}$ & 1.42552500 & 4.89052400 & 2.11333500 \\
\hline $\mathrm{H}$ & 2.88066400 & 1.85799400 & 0.15062600 \\
\hline $\mathrm{C}$ & 2.56454400 & 2.76557900 & 2.03904100 \\
\hline $\mathrm{C}$ & -0.22435500 & 7.01916300 & -3.40288600 \\
\hline $\mathrm{H}$ & 0.04470800 & 5.87565600 & -5.23094500 \\
\hline $\mathrm{H}$ & -0.38990600 & 7.87638600 & -1.45570400 \\
\hline $\mathrm{H}$ & 0.33673900 & 6.78488500 & 0.57708000 \\
\hline $\mathrm{C}$ & 2.03168900 & 3.86480700 & 2.77301500 \\
\hline
\end{tabular}




\begin{tabular}{|c|c|c|c|}
\hline $\mathrm{H}$ & 1.02885900 & 5.74640600 & 2.65220500 \\
\hline $\mathrm{H}$ & 3.05920600 & 1.96043100 & 2.57108900 \\
\hline $\mathrm{H}$ & -0.69772400 & 7.85426400 & -3.90809800 \\
\hline $\mathrm{H}$ & 2.12521100 & 3.88500600 & 3.85424900 \\
\hline C & -7.26241400 & -0.91829500 & -1.18593900 \\
\hline $\mathrm{C}$ & -7.12751700 & 0.17012600 & -0.33837600 \\
\hline $\mathrm{C}$ & -5.86818300 & 0.69212900 & -0.02020800 \\
\hline $\mathrm{C}$ & -4.76246600 & 0.04239600 & -0.56786400 \\
\hline $\mathrm{C}$ & -4.85462000 & -1.03111400 & -1.46812800 \\
\hline $\mathrm{C}$ & -6.13436200 & -1.50118300 & -1.76013900 \\
\hline $\mathrm{H}$ & -8.24931100 & -1.30426000 & -1.42084900 \\
\hline $\mathrm{H}$ & -8.01363000 & 0.63911700 & 0.08047000 \\
\hline $\mathrm{C}$ & -5.76718500 & 1.94761900 & 0.82867600 \\
\hline $\mathrm{C}$ & -3.60132500 & -1.59699600 & -2.11973100 \\
\hline $\mathrm{H}$ & -6.25834200 & -2.32912100 & -2.44989300 \\
\hline $\mathrm{C}$ & -6.47872600 & 3.11810800 & 0.13690000 \\
\hline $\mathrm{H}$ & -4.71253500 & 2.22105600 & 0.92439700 \\
\hline $\mathrm{C}$ & -6.32955800 & 1.72048000 & 2.23558900 \\
\hline $\mathrm{H}$ & -6.21670000 & 2.62639700 & 2.83985400 \\
\hline $\mathrm{H}$ & -5.80939800 & 0.89932000 & 2.73291500 \\
\hline $\mathrm{H}$ & -7.39784000 & 1.48192300 & 2.19265800 \\
\hline $\mathrm{H}$ & -6.35503400 & 4.03456700 & 0.72259200 \\
\hline $\mathrm{H}$ & -7.55256600 & 2.92699700 & 0.04146400 \\
\hline $\mathrm{H}$ & -6.07690000 & 3.29061500 & -0.86567900 \\
\hline $\mathrm{H}$ & -2.88740600 & -1.82396000 & -1.31858600 \\
\hline $\mathrm{C}$ & -2.95027700 & -0.54824100 & -3.03578400 \\
\hline $\mathrm{C}$ & -3.86377900 & -2.87238200 & -2.92031200 \\
\hline $\mathrm{H}$ & -2.04048100 & -0.95544200 & -3.49173900 \\
\hline
\end{tabular}




$\begin{array}{llll}\mathrm{H} & -2.67274400 & 0.34979900 & -2.48258500 \\ \mathrm{H} & -3.63719600 & -0.27043900 & -3.84298800 \\ \mathrm{H} & -4.46267500 & -2.66531900 & -3.81457800 \\ \mathrm{H} & -4.38215100 & -3.63363900 & -2.33107400 \\ \mathrm{H} & -2.91179400 & -3.30142000 & -3.25407300 \\ \mathrm{C} & 5.97734800 & -3.53697600 & -2.33129300 \\ \mathrm{H} & 5.38431100 & -3.00299500 & -3.08189600 \\ \mathrm{H} & 5.49086800 & -4.48266700 & -2.07164500 \\ \mathrm{H} & 6.97630700 & -3.72894500 & -2.71743000\end{array}$

\section{Structure syn-(2S,3R)-TS2 Precomplex}

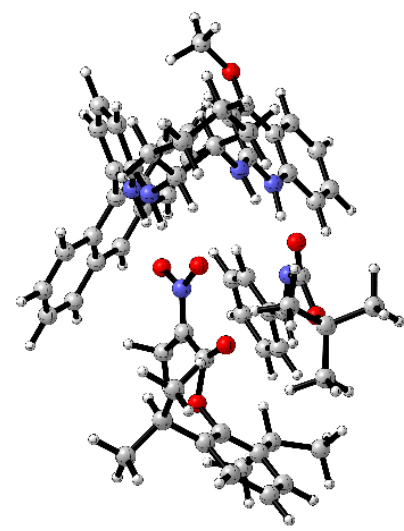

- Thermochemistry -
Zero-point correction $=$

Thermal correction to Energy=

Thermal correction to Enthalpy=

Thermal correction to Gibbs Free Energy=

Sum of electronic and zero-point Energies=

Sum of electronic and thermal Energies=

Sum of electronic and thermal Enthalpies=
1.222071 (Hartree/Particle)

1.291490

1.292434

1.115434

$-3256.506282$

$-3256.436863$

$-3256.435919$ 
Sum of electronic and thermal Free Energies $=\quad-3256.612920$

Number of Imaginary Frequencies $=0$

E (Single Point Energy) [SMD(Toluene)M06-2X/6-311++G(2d,2p)] = -3258.803853

$\begin{array}{lrrr}\mathrm{O} & 3.25173900 & 0.52001600 & 0.36394300 \\ \mathrm{C} & 3.00613200 & -0.59255800 & 0.77286900 \\ \mathrm{O} & 3.99875200 & -1.49333700 & 1.05190800 \\ \mathrm{C} & 1.69717900 & -1.19070100 & 1.03234000 \\ \mathrm{C} & 1.48612500 & -2.63200000 & 1.38154600 \\ \mathrm{H} & 1.02532600 & -2.72990600 & 2.37035800 \\ \mathrm{H} & 2.43635600 & -3.16323800 & 1.36949200 \\ \mathrm{H} & 0.79916700 & -3.09908700 & 0.66606900 \\ \mathrm{~N} & 0.61593300 & -0.41204900 & 0.99525000 \\ \mathrm{O} & 0.61999300 & 0.81862000 & 0.67988200 \\ \mathrm{O} & -0.51534900 & -0.95715700 & 1.28789600 \\ \mathrm{C} & 1.57109100 & -0.69437200 & -2.07581900 \\ \mathrm{C} & 0.80720200 & -1.94252500 & -2.02334700 \\ \mathrm{C} & 1.42225200 & -3.14071200 & -2.40779200 \\ \mathrm{C} & -0.51282500 & -1.95971400 & -1.55362700 \\ \mathrm{C} & 0.71535500 & -4.33630900 & -2.35476700 \\ \mathrm{C} & -1.21526500 & -3.15604000 & -1.49599200 \\ \mathrm{C} & -0.60404400 & -4.34218600 & -1.90285200 \\ \mathrm{C} & 1.79666600 & 1.62527000 & -2.04620300 \\ \mathrm{O} & 1.25368300 & 2.69793100 & -1.84075000 \\ \mathrm{O} & 3.06999700 & 1.43578200 & -2.27909800 \\ \mathrm{C} & 4.07183100 & 2.50934100 & -2.12567200 \\ \mathrm{C} & 3.80701300 & 3.37427700 & -0.89445600 \\ \mathrm{H} & 4.74460700 & 3.86939300 & -0.62265200 \\ \mathrm{H} & 3.48864100 & 2.74723900 & -0.05909600 \\ \mathrm{H} & 3.05048000 & 4.13602400 & -1.08495200 \\ \mathrm{C} & 4.05172600 & 3.32238700 & -3.41215800 \\ \mathrm{H} & 3.07552100 & 3.79636600 & -3.54854200 \\ \mathrm{H} & 4.26772300 & 2.68692900 & -4.27570000 \\ \mathrm{H} & 4.81228700 & 4.10713300 & -3.35989600 \\ \mathrm{C} & 5.36420100 & 1.72227800 & -1.95270800 \\ \mathrm{H} & 6.21483900 & 2.40925800 & -1.92588100 \\ \mathrm{H} & 5.50600300 & 1.02734700 & -2.78628800 \\ \mathrm{H} & 5.33282900 & 1.15451200 & -1.01734800 \\ \mathrm{~N} & 0.99752300 & 0.45326800 & -2.10311600 \\ \mathrm{H} & 2.66358000 & -0.76598800 & -2.08940600 \\ \mathrm{H} & -1.15332100 & -5.27759400 & -1.85456300 \\ \mathrm{H} & 1.19201200 & -5.26158500 & -2.66144900 \\ & & & \\ & & & \end{array}$




$\begin{array}{lrrr}\mathrm{H} & -2.23481000 & -3.16321100 & -1.12271000 \\ \mathrm{H} & -0.95878800 & -1.04571600 & -1.17579000 \\ \mathrm{H} & 2.44983600 & -3.12684400 & -2.76109800 \\ \mathrm{C} & -0.27522300 & 6.22503500 & 1.90873500 \\ \mathrm{C} & -1.05654500 & 6.52419300 & 0.62711800 \\ \mathrm{C} & -0.98038400 & 5.35433800 & -0.35711700 \\ \mathrm{C} & -1.46786800 & 4.05214000 & 0.28883100 \\ \mathrm{C} & -0.68918700 & 3.77395100 & 1.59070900 \\ \mathrm{C} & -0.78666400 & 4.94316900 & 2.57296100 \\ \mathrm{H} & 0.05730100 & 5.20435100 & -0.68525900 \\ \mathrm{H} & -0.67518000 & 7.43107100 & 0.14907600 \\ \mathrm{H} & -2.10771700 & 6.72045700 & 0.88039300 \\ \mathrm{H} & -0.35316000 & 7.06241800 & 2.60804500 \\ \mathrm{H} & 0.78970600 & 6.10889100 & 1.66845300 \\ \mathrm{H} & -2.53289400 & 4.14641800 & 0.52666400 \\ \mathrm{H} & -0.20998500 & 4.69902800 & 3.46981700 \\ \mathrm{H} & -1.82421700 & 5.10276400 & 2.89094800 \\ \mathrm{H} & -1.57354400 & 5.55223300 & -1.25573900 \\ \mathrm{~N} & -1.28197000 & 2.93041600 & -0.62026800 \\ \mathrm{H} & -0.31074600 & 2.76568300 & -0.92537800 \\ \mathrm{~N} & -1.00997200 & 2.45260400 & 2.13450800 \\ \mathrm{H} & -0.34551300 & 1.74928200 & 1.76765100 \\ \mathrm{C} & -2.25541500 & 1.96578600 & 2.29566400 \\ \mathrm{C} & -3.39806300 & 2.70373300 & 2.68321100 \\ \mathrm{C} & -3.56848700 & -0.04189500 & 2.29406700 \\ \mathrm{C} & -1.56342500 & 0.04642700 & 1.71938500 \\ \mathrm{H} & -4.58801000 & 2.03849200 & 2.88734000 \\ \mathrm{C} & -3.31330200 & 3.76544000 & 2.86800300 \\ \mathrm{C} & -4.69102900 & 0.65126500 & 2.70027000 \\ \mathrm{C} & -2.40824500 & 0.63195000 & 2.09576600 \\ \mathrm{~N} & -2.20717900 & 2.18364400 & -1.21704600 \\ \mathrm{C} & -3.61408300 & 2.29762200 & -0.99586800 \\ \mathrm{C} & -2.60277900 & 0.50954900 & -2.89690900 \\ \mathrm{C} & -4.47675700 & 1.53664000 & -1.74425500 \\ \mathrm{C} & -3.97501000 & 2.98260100 & -0.24341500 \\ \mathrm{C} & -3.99299700 & 0.64116500 & -2.76572100 \\ \mathrm{C} & -1.76580600 & 1.26022100 & -2.09516900 \\ \mathrm{C} & -2.05959200 & -0.36597100 & -3.85061200 \\ \mathrm{C} & -4.30669900 & -0.95550400 & -4.54573300 \\ \mathrm{C} & -31900800 & 0.07117000 & 1.03544900 \\ \mathrm{C} & -0.24298400 & 1.46491300 \\ \mathrm{C} & -1.09097200 & -4.65817300 \\ \mathrm{C} & -1.12135400 & 0.67067800 \\ \mathrm{C} & -1.6937400 & -1.35406100 & -0.89407500\end{array}$




\begin{tabular}{|c|c|c|c|}
\hline $\mathrm{H}$ & 8.84743800 & -0.21456400 & -0.44588900 \\
\hline $\mathrm{H}$ & 7.92180400 & 0.75531900 & 1.62686600 \\
\hline $\mathrm{C}$ & 5.46556800 & 0.35922900 & 2.73902600 \\
\hline $\mathrm{C}$ & 4.92219100 & -2.67454200 & -1.27974100 \\
\hline $\mathrm{H}$ & 7.50079900 & -1.77338500 & -1.80217900 \\
\hline $\mathrm{C}$ & 6.31884000 & -0.02897700 & 3.95236900 \\
\hline $\mathrm{H}$ & 4.46167500 & -0.04847300 & 2.89470000 \\
\hline $\mathrm{C}$ & 5.34645400 & 1.88172000 & 2.61377600 \\
\hline $\mathrm{H}$ & 4.90013200 & 2.30623900 & 3.51905300 \\
\hline $\mathrm{H}$ & 4.72378800 & 2.14748000 & 1.75567800 \\
\hline $\mathrm{H}$ & 6.33273600 & 2.34014300 & 2.48112700 \\
\hline $\mathrm{H}$ & 6.39730800 & -1.11530100 & 4.05085000 \\
\hline $\mathrm{H}$ & 5.87784900 & 0.37085000 & 4.87096400 \\
\hline $\mathrm{H}$ & 7.33273800 & 0.37604700 & 3.86674600 \\
\hline $\mathrm{H}$ & 3.88445600 & -2.32423800 & -1.20083000 \\
\hline $\mathrm{C}$ & 4.97094300 & -4.06060900 & -0.62298700 \\
\hline $\mathrm{C}$ & 5.28860000 & -2.76208300 & -2.76070900 \\
\hline $\mathrm{H}$ & 4.28708300 & -4.75188000 & -1.12850700 \\
\hline $\mathrm{H}$ & 4.68850000 & -4.00359600 & 0.43185500 \\
\hline $\mathrm{H}$ & 5.98359200 & -4.47333500 & -0.68345000 \\
\hline $\mathrm{H}$ & 6.28275800 & -3.19888100 & -2.89934600 \\
\hline $\mathrm{H}$ & 5.27961300 & -1.77716300 & -3.23944400 \\
\hline $\mathrm{H}$ & 4.58555400 & -3.41281100 & -3.29176400 \\
\hline $\mathrm{H}$ & 0.36798700 & 3.68100400 & 1.30468900 \\
\hline $\mathrm{H}$ & -0.74371300 & 1.05077300 & -2.13979000 \\
\hline $\mathrm{O}$ & -5.80386600 & 1.54881200 & -1.60442400 \\
\hline $\mathrm{C}$ & -6.37545000 & 2.40182700 & -0.62189100 \\
\hline $\mathrm{H}$ & -6.00320400 & 2.13887900 & 0.37524500 \\
\hline $\mathrm{H}$ & -6.14782500 & 3.44940100 & -0.84288400 \\
\hline $\mathrm{H}$ & -7.44985900 & 2.23675800 & -0.67344700 \\
\hline $\mathrm{C}$ & -4.84262000 & -0.09681900 & -3.61027000 \\
\hline $\mathrm{H}$ & -0.98146100 & -0.45505500 & -3.93923700 \\
\hline $\mathrm{H}$ & -2.49448800 & -1.76721700 & -5.39709900 \\
\hline $\mathrm{H}$ & -4.95892300 & -1.52204900 & -5.20092500 \\
\hline $\mathrm{H}$ & -5.91491000 & 0.03278100 & -3.51820200 \\
\hline $\mathrm{C}$ & -3.53373500 & -1.50251600 & 2.03237000 \\
\hline $\mathrm{C}$ & -2.70155800 & -2.32858300 & 2.81450500 \\
\hline $\mathrm{C}$ & -4.31645300 & -2.02848900 & 0.98438600 \\
\hline $\mathrm{C}$ & -1.92830500 & -1.84279000 & 3.91764400 \\
\hline $\mathrm{C}$ & -2.63657900 & -3.73011500 & 2.51401200 \\
\hline $\mathrm{C}$ & -4.26093900 & -3.43899300 & 0.71925200 \\
\hline $\mathrm{C}$ & -5.15179400 & -1.22541500 & 0.14222300 \\
\hline $\mathrm{C}$ & -1.13665400 & -2.68308300 & 4.64276200 \\
\hline $\mathrm{H}$ & -1.98235000 & -0.79357300 & 4.18888900 \\
\hline $\mathrm{C}$ & -1.78276400 & -4.57536200 & 3.29072300 \\
\hline $\mathrm{C}$ & -3.41764400 & -4.24783800 & 1.48161400 \\
\hline
\end{tabular}




$\begin{array}{lrrr}\mathrm{C} & -5.05266200 & -3.98470500 & -0.34075800 \\ \mathrm{H} & -5.19062400 & -0.15300900 & 0.30058200 \\ \mathrm{C} & -5.89512200 & -1.78435600 & -0.85528400 \\ \mathrm{C} & -1.05095800 & -4.07022600 & 4.32184700 \\ \mathrm{H} & -0.56375700 & -2.29523600 & 5.47900000 \\ \mathrm{H} & -1.74220100 & -5.63216400 & 3.04233800 \\ \mathrm{H} & -3.37071500 & -5.31346400 & 1.26661700 \\ \mathrm{C} & -5.85021000 & -3.18677200 & -1.10473400 \\ \mathrm{H} & -5.00119100 & -5.05535300 & -0.51958300 \\ \mathrm{H} & -6.52572000 & -1.14824000 & -1.46848000 \\ \mathrm{H} & -0.40982600 & -4.71747600 & 4.91100100 \\ \mathrm{H} & -6.45010100 & -3.61031800 & -1.90336700 \\ \mathrm{H} & -5.61587200 & 0.11554400 & 2.87569800 \\ \mathrm{H} & -5.45641900 & 2.59935600 & 3.22117200\end{array}$

\section{Structure anti-(2R,3S)-TS2 Precomplex}

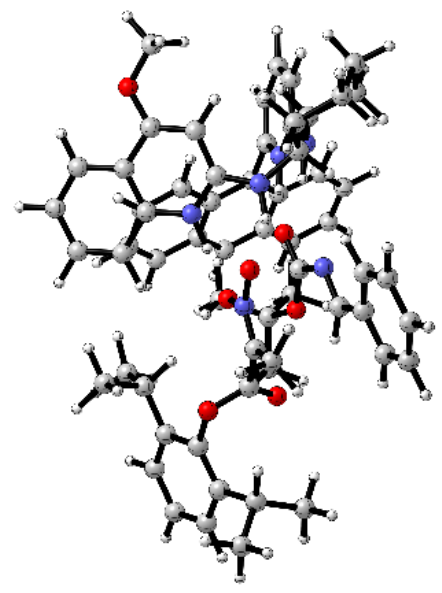

- Thermochemistry -

Zero-point correction=

Thermal correction to Energy=

Thermal correction to Enthalpy=

Thermal correction to Gibbs Free Energy=

Sum of electronic and zero-point Energies=
1.222572 (Hartree/Particle)

1.292021

1.292966

1.116957

$-3256.497313$ 
Sum of electronic and thermal Energies=

$-3256.427864$

Sum of electronic and thermal Enthalpies=

$-3256.426920$

Sum of electronic and thermal Free Energies=

$-3256.602929$

Number of Imaginary Frequencies $=0$

E (Single Point Energy) [SMD(Toluene)M06-2X/6-311++G(2d,2p)] = -3258.798629

\begin{tabular}{|c|c|c|c|}
\hline $\mathrm{O}$ & 3.92812400 & -0.24317400 & -1.16688000 \\
\hline $\mathrm{C}$ & 4.05670500 & 0.27744300 & -0.08689300 \\
\hline $\mathrm{O}$ & 4.76886900 & -0.26076000 & 0.92545500 \\
\hline $\mathrm{C}$ & 3.53642700 & 1.62672600 & 0.28016600 \\
\hline $\mathrm{C}$ & 4.42180000 & 2.82665500 & 0.27371400 \\
\hline $\mathrm{H}$ & 4.97529100 & 2.91211500 & 1.21641900 \\
\hline $\mathrm{H}$ & 3.81762000 & 3.72652700 & 0.14055000 \\
\hline $\mathrm{H}$ & 5.14691600 & 2.75858200 & -0.54313000 \\
\hline $\mathrm{N}$ & 2.28917200 & 1.68598500 & 0.66061900 \\
\hline $\mathrm{O}$ & 1.70821600 & 2.78500400 & 0.95051000 \\
\hline $\mathrm{O}$ & 1.57985900 & 0.58193000 & 0.72399200 \\
\hline $\mathrm{C}$ & 1.79576800 & 1.81091000 & -2.20080600 \\
\hline $\mathrm{C}$ & 2.45478000 & 3.10123000 & -2.37703600 \\
\hline $\mathrm{C}$ & 1.90624300 & 4.28753900 & -1.87381700 \\
\hline $\mathrm{C}$ & 3.69697800 & 3.12857800 & -3.02229500 \\
\hline $\mathrm{C}$ & 2.58739100 & 5.48639700 & -2.03113300 \\
\hline $\mathrm{C}$ & 4.37353000 & 4.33194000 & -3.18671400 \\
\hline $\mathrm{C}$ & 3.81723800 & 5.51002500 & -2.69197800 \\
\hline $\mathrm{C}$ & 0.10211700 & 0.41081100 & -1.54667800 \\
\hline $\mathrm{O}$ & -1.04407100 & 0.26832800 & -1.11573500 \\
\hline C & 0.89273800 & -0.58045600 & -1.87489600 \\
\hline
\end{tabular}




\begin{tabular}{|c|c|c|c|}
\hline $\mathrm{C}$ & 0.56844000 & -1.98257000 & -1.57721400 \\
\hline $\mathrm{C}$ & 0.22424100 & -2.15179600 & -0.10292800 \\
\hline $\mathrm{H}$ & -0.72840600 & -1.69008000 & 0.16230000 \\
\hline $\mathrm{H}$ & 0.16109500 & -3.22155000 & 0.11864100 \\
\hline $\mathrm{C}$ & 1.87232800 & -2.70209700 & -1.89889200 \\
\hline $\mathrm{H}$ & 2.67815500 & -2.34525600 & -1.25081100 \\
\hline $\mathrm{H}$ & 2.16348100 & -2.52318600 & -2.93796000 \\
\hline $\mathrm{H}$ & 1.74138900 & -3.77843200 & -1.75249300 \\
\hline $\mathrm{C}$ & -0.55239400 & -2.43981600 & -2.50328500 \\
\hline $\mathrm{H}$ & -0.70708100 & -3.51577800 & -2.37751000 \\
\hline $\mathrm{H}$ & -0.28769500 & -2.24923500 & -3.54751600 \\
\hline $\mathrm{H}$ & -1.49645000 & -1.93699900 & -2.27761100 \\
\hline $\mathrm{N}$ & 0.59228400 & 1.70915200 & -1.74976600 \\
\hline $\mathrm{H}$ & 2.38631500 & 0.92598700 & -2.44981900 \\
\hline $\mathrm{H}$ & 4.34665400 & 6.44993100 & -2.81312100 \\
\hline $\mathrm{H}$ & 2.16926500 & 6.40589200 & -1.63389600 \\
\hline $\mathrm{H}$ & 5.33344500 & 4.35248800 & -3.69203400 \\
\hline $\mathrm{H}$ & 4.12963400 & 2.19767300 & -3.38171200 \\
\hline $\mathrm{H}$ & 0.96730300 & 4.24149000 & -1.33401700 \\
\hline $\mathrm{C}$ & -2.33416300 & 6.34082500 & -0.29493600 \\
\hline $\mathrm{C}$ & -3.69741900 & 5.92441800 & -0.85556300 \\
\hline $\mathrm{C}$ & -3.64027500 & 4.53292200 & -1.49491200 \\
\hline $\mathrm{C}$ & -3.14118600 & 3.50824500 & -0.47012100 \\
\hline $\mathrm{C}$ & -1.73160300 & 3.92196800 & -0.00254100 \\
\hline $\mathrm{C}$ & -1.77737800 & 5.29457000 & 0.67657100 \\
\hline $\mathrm{H}$ & -2.95764000 & 4.53061000 & -2.35381200 \\
\hline $\mathrm{H}$ & -4.04613400 & 6.65621000 & -1.58976000 \\
\hline $\mathrm{H}$ & -4.43761400 & 5.91708700 & -0.04312000 \\
\hline
\end{tabular}




\begin{tabular}{|c|c|c|c|}
\hline $\mathrm{H}$ & -2.40889900 & 7.31201900 & 0.20263000 \\
\hline $\mathrm{H}$ & -1.62615100 & 6.46436300 & -1.12535700 \\
\hline $\mathrm{H}$ & -3.83619600 & 3.51823200 & 0.38130300 \\
\hline $\mathrm{H}$ & -0.76650200 & 5.55840000 & 1.00241400 \\
\hline $\mathrm{H}$ & -2.40181300 & 5.24285000 & 1.57931900 \\
\hline $\mathrm{H}$ & -4.62516100 & 4.23752900 & -1.87353900 \\
\hline $\mathrm{N}$ & -3.06115400 & 2.14631300 & -0.97478000 \\
\hline $\mathrm{H}$ & -2.11892200 & 1.73970300 & -1.06856700 \\
\hline $\mathrm{N}$ & -1.01354200 & 2.89273300 & 0.73131300 \\
\hline $\mathrm{H}$ & -0.01268100 & 2.77319700 & 0.48488500 \\
\hline $\mathrm{C}$ & -1.41374700 & 2.13516300 & 1.75650100 \\
\hline $\mathrm{C}$ & -2.62224600 & 2.32585800 & 2.49205200 \\
\hline $\mathrm{C}$ & -0.83720300 & 0.29071000 & 3.15937100 \\
\hline $\mathrm{H}$ & 0.29932900 & 0.95463300 & 1.52168800 \\
\hline $\mathrm{C}$ & -2.90769900 & 1.49863600 & 3.55046900 \\
\hline $\mathrm{H}$ & -3.24559000 & 3.17450900 & 2.25892500 \\
\hline $\mathrm{C}$ & 0.09898900 & -0.71176400 & 3.47706400 \\
\hline $\mathrm{C}$ & -2.00158800 & 0.44518100 & 3.93216900 \\
\hline $\mathrm{O}$ & -3.99855400 & 1.60146200 & 4.31183500 \\
\hline $\mathrm{C}$ & -0.13216100 & -1.52203900 & 4.56859000 \\
\hline $\mathrm{H}$ & 0.99240500 & -0.80427400 & 2.86387400 \\
\hline $\mathrm{C}$ & -2.21526800 & -0.39361900 & 5.04479500 \\
\hline $\mathrm{C}$ & -1.28779200 & -1.36382100 & 5.36089100 \\
\hline $\mathrm{H}$ & 0.59424100 & -2.28769700 & 4.82598600 \\
\hline $\mathrm{H}$ & -3.10940500 & -0.25552700 & 5.64223700 \\
\hline $\mathrm{H}$ & -1.44420300 & -2.00472300 & 6.22163700 \\
\hline $\mathrm{N}$ & -0.60027700 & 1.12119000 & 2.09324500 \\
\hline $\mathrm{C}$ & -4.06878800 & 1.27378500 & -0.92772000 \\
\hline
\end{tabular}




\begin{tabular}{|c|c|c|c|}
\hline $\mathrm{C}$ & -5.43206200 & 1.60200900 & -0.72553000 \\
\hline $\mathrm{C}$ & -4.67749000 & -1.05743400 & -1.07017200 \\
\hline C & -6.37009900 & 0.59651300 & -0.72752000 \\
\hline $\mathrm{H}$ & -5.71707000 & 2.63914900 & -0.60524400 \\
\hline $\mathrm{C}$ & -6.00607900 & -0.75293200 & -0.90478400 \\
\hline $\mathrm{H}$ & -7.41594200 & 0.85044900 & -0.58510300 \\
\hline $\mathrm{H}$ & -6.73773600 & -1.55058000 & -0.88870300 \\
\hline $\mathrm{N}$ & -3.76690700 & -0.04370600 & -1.08712400 \\
\hline $\mathrm{C}$ & -4.11470800 & -2.42573400 & -1.17058200 \\
\hline $\mathrm{C}$ & -4.27848900 & -3.17211400 & -2.35206200 \\
\hline $\mathrm{C}$ & -3.40129000 & -2.93004000 & -0.06475300 \\
\hline $\mathrm{C}$ & -4.97752900 & -2.67882800 & -3.49996400 \\
\hline $\mathrm{C}$ & -3.69686800 & -4.48337500 & -2.42276400 \\
\hline $\mathrm{C}$ & -2.82035900 & -4.23939600 & -0.15727000 \\
\hline $\mathrm{C}$ & -3.25546300 & -2.21250000 & 1.16713700 \\
\hline $\mathrm{C}$ & -5.10127900 & -3.44331600 & -4.62283600 \\
\hline $\mathrm{H}$ & -5.41034100 & -1.68373200 & -3.47220000 \\
\hline $\mathrm{C}$ & -3.85203300 & -5.25075500 & -3.61981600 \\
\hline $\mathrm{C}$ & -2.98526200 & -4.97911800 & -1.32944500 \\
\hline $\mathrm{C}$ & -2.09572800 & -4.76283100 & 0.96005000 \\
\hline $\mathrm{H}$ & -3.71306500 & -1.23313800 & 1.27869700 \\
\hline $\mathrm{C}$ & -2.56093100 & -2.75005500 & 2.21094900 \\
\hline $\mathrm{C}$ & -4.53466500 & -4.75034900 & -4.68698700 \\
\hline $\mathrm{H}$ & -5.63533600 & -3.05384100 & -5.48359000 \\
\hline $\mathrm{H}$ & -3.41036300 & -6.24259400 & -3.65564800 \\
\hline $\mathrm{H}$ & -2.54681500 & -5.97295600 & -1.39251600 \\
\hline $\mathrm{C}$ & -1.96165900 & -4.03918300 & 2.10701900 \\
\hline $\mathrm{H}$ & -1.65894500 & -5.75377000 & 0.87036100 \\
\hline
\end{tabular}




\begin{tabular}{|c|c|c|c|}
\hline $\mathrm{H}$ & -2.47245100 & -2.19794200 & 3.14053400 \\
\hline $\mathrm{H}$ & -4.64843600 & -5.33829300 & -5.59144000 \\
\hline $\mathrm{H}$ & -1.41077900 & -4.44023600 & 2.95187600 \\
\hline C & 6.33629200 & -4.06662100 & 0.41516100 \\
\hline C & 6.98345900 & -2.98034900 & -0.15827100 \\
\hline C & 6.47828900 & -1.68353200 & -0.02264000 \\
\hline C & 5.30358700 & -1.54118400 & 0.71164200 \\
\hline C & 4.61782600 & -2.60780900 & 1.30082100 \\
\hline C & 5.16192900 & -3.88118700 & 1.14078300 \\
\hline $\mathrm{H}$ & 6.74670800 & -5.06452400 & 0.29780400 \\
\hline $\mathrm{H}$ & 7.89661300 & -3.13690800 & -0.72611500 \\
\hline C & 7.18096300 & -0.50219900 & -0.66473200 \\
\hline $\mathrm{C}$ & 3.36322000 & -2.33548000 & 2.11084700 \\
\hline $\mathrm{H}$ & 4.66477200 & -4.73880200 & 1.58290900 \\
\hline C & 8.63892800 & -0.39899500 & -0.20302000 \\
\hline $\mathrm{H}$ & 6.67024900 & 0.41215800 & -0.34275300 \\
\hline $\mathrm{C}$ & 7.09179600 & -0.58670500 & -2.19335400 \\
\hline $\mathrm{H}$ & 7.55637200 & 0.29253300 & -2.65213300 \\
\hline $\mathrm{H}$ & 6.04992300 & -0.64868000 & -2.51813500 \\
\hline $\mathrm{H}$ & 7.61953700 & -1.47356400 & -2.56106200 \\
\hline $\mathrm{H}$ & 9.10927200 & 0.49204100 & -0.63047300 \\
\hline $\mathrm{H}$ & 9.21948300 & -1.26795300 & -0.52973400 \\
\hline $\mathrm{H}$ & 8.70708300 & -0.33722400 & 0.88661100 \\
\hline $\mathrm{H}$ & 2.81488900 & -1.53267800 & 1.59909700 \\
\hline $\mathrm{C}$ & 3.74711700 & -1.81602800 & 3.50422500 \\
\hline C & 2.44504700 & -3.55319400 & 2.21934200 \\
\hline $\mathrm{H}$ & 2.85481900 & -1.59040000 & 4.10011500 \\
\hline $\mathrm{H}$ & 4.34510300 & -0.90412300 & 3.43037400 \\
\hline
\end{tabular}




$\begin{array}{llll}\mathrm{H} & 4.33208000 & -2.57044500 & 4.04129800 \\ \mathrm{H} & 2.87321200 & -4.31884600 & 2.87579400 \\ \mathrm{H} & 2.26106400 & -4.00595200 & 1.23943100 \\ \mathrm{H} & 1.47546100 & -3.26018300 & 2.64017000 \\ \mathrm{C} & -4.93994500 & 2.61960800 & 4.00937500 \\ \mathrm{H} & -5.75211700 & 2.49672300 & 4.72313900 \\ \mathrm{H} & -5.31876800 & 2.49964500 & 2.98781700 \\ \mathrm{H} & -4.48939400 & 3.61032500 & 4.12744900 \\ \mathrm{H} & -1.14251400 & 4.02223100 & -0.92458900 \\ \mathrm{H} & -2.76881800 & -0.28525000 & -1.17375900 \\ \mathrm{H} & 1.01628800 & -1.70968200 & 0.50745100\end{array}$

\section{Structure syn-(2S,3S)-TS2 Precomplex}

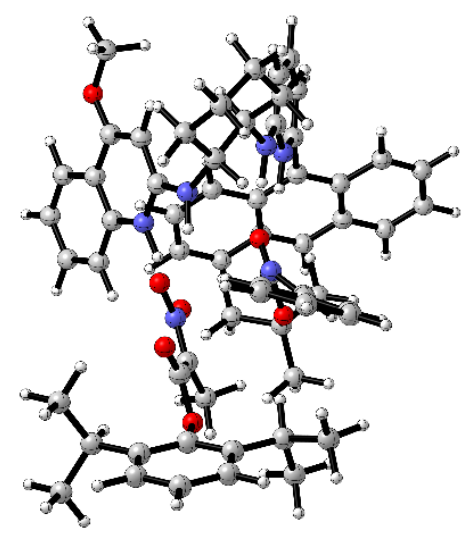

- Thermochemistry -

Zero-point correction $=$ Thermal correction to Energy= Thermal correction to Enthalpy=
1.222881 (Hartree/Particle)

1.292488

1.293432 
Thermal correction to Gibbs Free Energy=

Sum of electronic and zero-point Energies $=$

1.115461

Sum of electronic and thermal Energies $=$

$-3256.494749$

Sum of electronic and thermal Enthalpies=

$-3256.425142$

$-3256.424198$

Sum of electronic and thermal Free Energies=

$-3256.602169$

Number of Imaginary Frequencies $=0$

E (Single Point Energy) [SMD(Toluene)M06-2X/6-311++G(2d,2p)] = -3258.798883

$\begin{array}{llll}\mathrm{O} & -4.68367000 & 0.99595800 & -0.22975800 \\ \mathrm{C} & -4.37526500 & 0.02705900 & -0.87903000 \\ \mathrm{O} & -5.28840400 & -0.92264900 & -1.25293100 \\ \mathrm{C} & -3.05125900 & -0.35081800 & -1.39568600 \\ \mathrm{C} & -2.78860400 & -1.59308600 & -2.19176700 \\ \mathrm{H} & -2.30943400 & -1.34419900 & -3.14440300 \\ \mathrm{H} & -3.71910500 & -2.12183600 & -2.38634100 \\ \mathrm{H} & -2.10288900 & -2.25472100 & -1.65015700 \\ \mathrm{~N} & -2.01855300 & 0.45380900 & -1.17844400 \\ \mathrm{O} & -0.86120700 & 0.11993500 & -1.66293300 \\ \mathrm{O} & -2.07892500 & 1.53251800 & -0.51326500 \\ \mathrm{C} & -1.42532800 & -1.74333900 & 1.56138700 \\ \mathrm{C} & -2.60391600 & -1.35455800 & 2.32708600 \\ \mathrm{C} & -3.17918600 & -2.27059300 & 3.21818900 \\ \mathrm{C} & -3.18533000 & -0.09371300 & 2.13543900 \\ \mathrm{C} & -4.30424600 & -1.91021000 & 3.94798700 \\ \mathrm{C} & -4.34091700 & 0.24020400 & 2.83103400 \\ \mathrm{C} & -4.88736500 & -0.65785200 & 3.74784400 \\ \mathrm{C} & 0.41176700 & -1.32108400 & 0.23549000 \\ \mathrm{O} & 1.47607200 & -0.70446700 & 0.22879500\end{array}$




\begin{tabular}{|c|c|c|c|}
\hline $\mathrm{O}$ & 0.16590200 & -2.41082000 & -0.45489400 \\
\hline $\mathrm{C}$ & 1.12910700 & -2.95244100 & -1.42966800 \\
\hline $\mathrm{C}$ & 2.35060100 & -3.48501200 & -0.69007000 \\
\hline $\mathrm{H}$ & 2.93958400 & -2.67999900 & -0.24488800 \\
\hline $\mathrm{H}$ & 2.99377800 & -4.01602600 & -1.39815800 \\
\hline $\mathrm{H}$ & 2.04899000 & -4.18856200 & 0.09205800 \\
\hline $\mathrm{C}$ & 1.47190100 & -1.89204500 & -2.46955200 \\
\hline $\mathrm{H}$ & 0.54976100 & -1.45642400 & -2.86569300 \\
\hline $\mathrm{H}$ & 2.02534600 & -2.36925600 & -3.28441800 \\
\hline $\mathrm{H}$ & 2.09148300 & -1.09401100 & -2.05324600 \\
\hline $\mathrm{C}$ & 0.34735300 & -4.09599600 & -2.06375100 \\
\hline $\mathrm{H}$ & -0.53316800 & -3.71436900 & -2.58719200 \\
\hline $\mathrm{H}$ & 0.02299100 & -4.81070200 & -1.30207100 \\
\hline $\mathrm{H}$ & 0.98144100 & -4.61906800 & -2.78456000 \\
\hline $\mathrm{N}$ & -0.64210700 & -0.87402400 & 1.03280900 \\
\hline $\mathrm{H}$ & -1.23014600 & -2.81748900 & 1.45760600 \\
\hline $\mathrm{H}$ & -5.78776300 & -0.39175000 & 4.29345300 \\
\hline $\mathrm{H}$ & -4.74551800 & -2.61024300 & 4.65003000 \\
\hline $\mathrm{H}$ & -4.82703800 & 1.18866700 & 2.62952400 \\
\hline $\mathrm{H}$ & -2.77557200 & 0.58892300 & 1.39339000 \\
\hline $\mathrm{H}$ & -2.73687300 & -3.25627600 & 3.34142300 \\
\hline $\mathrm{C}$ & -0.73531900 & 3.61816700 & 4.38035300 \\
\hline $\mathrm{C}$ & 0.57671500 & 3.29696900 & 5.10314700 \\
\hline $\mathrm{C}$ & 1.21988400 & 2.01404700 & 4.56343500 \\
\hline $\mathrm{C}$ & 1.44437400 & 2.14470700 & 3.05326700 \\
\hline $\mathrm{C}$ & 0.08148600 & 2.36680100 & 2.36981800 \\
\hline C & -0.54777800 & 3.67437200 & 2.86034400 \\
\hline $\mathrm{H}$ & 0.56959400 & 1.14977700 & 4.74719400 \\
\hline
\end{tabular}




\begin{tabular}{|c|c|c|c|}
\hline $\mathrm{H}$ & 0.40425300 & 3.19999200 & 6.17878500 \\
\hline $\mathrm{H}$ & 1.27862100 & 4.13249600 & 4.97174900 \\
\hline H & -1.14453400 & 4.56553400 & 4.74265500 \\
\hline $\mathrm{H}$ & -1.47662600 & 2.84286900 & 4.61442400 \\
\hline H & 2.09143300 & 3.01739100 & 2.88338500 \\
\hline F & -0.56299500 & 1.54202600 & 2.70713000 \\
\hline $\mathrm{H}$ & -1.50606500 & 3.81465600 & 2.35167600 \\
\hline $\mathrm{H}$ & 0.09286700 & 4.52375200 & 2.58475200 \\
\hline $\mathrm{H}$ & 2.16887600 & 1.81092500 & 5.07226400 \\
\hline r & 2.07636900 & 0.98670100 & 2.43507700 \\
\hline $\mathrm{H}$ & 1.51413300 & 0.47283100 & 1.74661300 \\
\hline 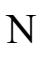 & 0.10398200 & 2.18823400 & 0.92832600 \\
\hline $\mathrm{H}$ & -0.70427900 & 1.67314200 & 0.52899300 \\
\hline C & 0.92467500 & 2.69021000 & 0.00337300 \\
\hline C & 1.91406100 & 3.69236600 & 0.23360400 \\
\hline C & 1.50964700 & 2.62384600 & -2.31312400 \\
\hline $\mathrm{H}$ & 0.08584600 & 1.39394400 & -1.38898600 \\
\hline $\mathrm{C}$ & 2.67740400 & 4.14140400 & -0.81662600 \\
\hline $\mathrm{H}$ & 1.98227200 & 4.14126300 & 1.21221300 \\
\hline C & 1.25682200 & 2.07608000 & -3.58436300 \\
\hline C & 2.48777300 & 3.62026800 & -2.14751600 \\
\hline $\mathrm{O}$ & 3.61610100 & 5.08326600 & -0.71425600 \\
\hline $\mathrm{C}$ & 1.97037300 & 2.54412500 & -4.66732200 \\
\hline $\mathrm{H}$ & 0.49241500 & 1.31007100 & -3.67919400 \\
\hline C & 3.20551000 & 4.08133900 & -3.26933800 \\
\hline C & 2.94653700 & 3.54992300 & -4.51537000 \\
\hline $\mathrm{H}$ & 1.77057500 & 2.13665500 & -5.65326500 \\
\hline $\mathrm{H}$ & 3.94967000 & 4.85806100 & -3.13380000 \\
\hline
\end{tabular}




\begin{tabular}{|c|c|c|c|}
\hline $\mathrm{H}$ & 3.49042000 & 3.90929000 & -5.38200400 \\
\hline $\mathrm{N}$ & 0.79019100 & 2.18069700 & -1.23302700 \\
\hline $\mathrm{C}$ & 3.39846600 & 0.82075300 & 2.34492200 \\
\hline $\mathrm{C}$ & 4.37183100 & 1.52248900 & 3.09628200 \\
\hline $\mathrm{C}$ & 5.16382900 & -0.40185500 & 1.24034700 \\
\hline $\mathrm{H}$ & 3.14680000 & -0.56983200 & 0.86984600 \\
\hline $\mathrm{C}$ & 5.70216200 & 1.22571600 & 2.90736000 \\
\hline $\mathrm{H}$ & 4.05587500 & 2.25990300 & 3.82261500 \\
\hline $\mathrm{C}$ & 6.11992000 & 0.25415500 & 1.97688500 \\
\hline $\mathrm{H}$ & 6.44819300 & 1.75858400 & 3.48879300 \\
\hline $\mathrm{H}$ & 7.16651600 & 0.03043900 & 1.81383700 \\
\hline $\mathrm{N}$ & 3.85229500 & -0.10403300 & 1.45791600 \\
\hline $\mathrm{C}$ & 5.41800300 & -1.39478300 & 0.16988100 \\
\hline $\mathrm{C}$ & 5.91569800 & -2.67064400 & 0.49774800 \\
\hline $\mathrm{C}$ & 5.11015400 & -1.03525600 & -1.15928400 \\
\hline $\mathrm{C}$ & 6.21226600 & -3.07900200 & 1.83788800 \\
\hline $\mathrm{C}$ & 6.11179000 & -3.62421700 & -0.55849700 \\
\hline $\mathrm{C}$ & 5.30510600 & -2.00596000 & -2.19871300 \\
\hline $\mathrm{C}$ & 4.64923600 & 0.26879500 & -1.53479300 \\
\hline $\mathrm{C}$ & 6.68917000 & -4.33040300 & 2.09748600 \\
\hline $\mathrm{H}$ & 6.05126600 & -2.38319600 & 2.65490500 \\
\hline $\mathrm{C}$ & 6.61531300 & -4.92497800 & -0.24111800 \\
\hline $\mathrm{C}$ & 5.80022600 & -3.26907000 & -1.87122200 \\
\hline $\mathrm{C}$ & 5.00331700 & -1.65372500 & -3.55201700 \\
\hline $\mathrm{H}$ & 4.52710300 & 1.03817000 & -0.77740100 \\
\hline $\mathrm{C}$ & 4.38905700 & 0.56953100 & -2.84005200 \\
\hline $\mathrm{C}$ & 6.90003600 & -5.26939500 & 1.04536800 \\
\hline $\mathrm{H}$ & 6.90965700 & -4.62084300 & 3.1196870 \\
\hline
\end{tabular}




\begin{tabular}{|c|c|c|c|}
\hline $\mathrm{H}$ & 6.76433900 & -5.63060800 & -1.05349600 \\
\hline $\mathrm{H}$ & 5.95099700 & -3.99849400 & -2.66444300 \\
\hline $\mathrm{C}$ & 4.55450900 & -0.40611200 & -3.86548300 \\
\hline $\mathrm{H}$ & 5.15218200 & -2.40445200 & -4.32339100 \\
\hline $\mathrm{H}$ & 4.06619700 & 1.56982200 & -3.10789600 \\
\hline $\mathrm{H}$ & 7.28295200 & -6.25711500 & 1.27857600 \\
\hline $\mathrm{H}$ & 4.33194600 & -0.14075400 & -4.89390800 \\
\hline $\mathrm{C}$ & -9.25849000 & -0.46685000 & -0.14992100 \\
\hline $\mathrm{C}$ & -8.45526700 & -1.40929500 & 0.48725000 \\
\hline $\mathrm{C}$ & -7.11172000 & -1.55458900 & 0.14389200 \\
\hline $\mathrm{C}$ & -6.61530300 & -0.72314900 & -0.86808600 \\
\hline $\mathrm{C}$ & -7.39033300 & 0.22567000 & -1.53405800 \\
\hline $\mathrm{C}$ & -8.72907700 & 0.33940700 & -1.14725600 \\
\hline $\mathrm{H}$ & -10.30132600 & -0.36544100 & 0.13385400 \\
\hline $\mathrm{H}$ & -8.88365400 & -2.03288600 & 1.26463300 \\
\hline $\mathrm{C}$ & -6.20060600 & -2.58289500 & 0.79378600 \\
\hline $\mathrm{C}$ & -6.82919300 & 1.10821500 & -2.63369200 \\
\hline $\mathrm{H}$ & -9.36226700 & 1.07284900 & -1.63948600 \\
\hline $\mathrm{C}$ & -6.78045900 & -3.16959800 & 2.08007900 \\
\hline $\mathrm{H}$ & -5.25789100 & -2.07374200 & 1.04515900 \\
\hline $\mathrm{C}$ & -5.88064700 & -3.72164800 & -0.18632100 \\
\hline $\mathrm{H}$ & -5.18548900 & -4.43494600 & 0.27038300 \\
\hline $\mathrm{H}$ & -5.43461100 & -3.34322300 & -1.10833300 \\
\hline $\mathrm{H}$ & -6.79810200 & -4.26082600 & -0.44600700 \\
\hline $\mathrm{H}$ & -6.04108400 & -3.82080200 & 2.55681700 \\
\hline $\mathrm{H}$ & -7.66710200 & -3.77805700 & 1.87020800 \\
\hline $\mathrm{H}$ & -7.06076400 & -2.38992700 & 2.79531500 \\
\hline C & -6.79068200 & 2.57439900 & -2.18751200 \\
\hline
\end{tabular}




$\begin{array}{llll}\mathrm{C} & -7.62618100 & 0.94936300 & -3.93387600 \\ \mathrm{H} & -6.35517000 & 3.20050300 & -2.97332900 \\ \mathrm{H} & -6.19468200 & 2.68389200 & -1.27850800 \\ \mathrm{H} & -7.80347500 & 2.94243500 & -1.98826700 \\ \mathrm{H} & -8.65771200 & 1.29577200 & -3.81091700 \\ \mathrm{H} & -7.65782800 & -0.09551000 & -4.25580400 \\ \mathrm{H} & -7.17094300 & 1.54439800 & -4.73204500 \\ \mathrm{C} & 3.85498100 & 5.66404300 & 0.55886800 \\ \mathrm{H} & 4.15292100 & 4.89500700 & 1.28079900 \\ \mathrm{H} & 2.96363600 & 6.18876000 & 0.91728300 \\ \mathrm{H} & 4.66776200 & 6.37320600 & 0.41543600 \\ \mathrm{H} & -5.80229900 & 0.78844000 & -2.83905100\end{array}$


Parallel Investigation of $\boldsymbol{C}_{2}$-Symmetric PBAM-Catalyzed anti-Selective aza-Henry Reactions With respect to the activation barriers of the diastereodivergent cases $($ anti- $(2 R, 3 R), \boldsymbol{s y n}-(\mathbf{2 S}, \mathbf{3 R})$, anti-(2S,3S), syn-(2R,3S)) arising from the use of $C_{2}$-symmetrical catalyst 3 , it can be seen that similar activation barriers were observed, respectively, corresponding to $8.8 \mathrm{kcal} \mathrm{mol}^{-1}, 11.8 \mathrm{kcal}$ $\mathrm{mol}^{-1}, 14.2 \mathrm{kcal} \mathrm{mol}^{-1}$ and $9.5 \mathrm{kcal} \mathrm{mol}^{-1}$. The most energetically favorable of these structures being anti-(2R,3R)-TS, displaying a carbon-carbon bond forming distance of $2.21 \AA$ (Figure S1). Moreover, this transition state exhibited two different hydrogen bonding substrate activation modes for each respective substrate. Two-point hydrogen bonding was found to persist as the dominant binding mode allowing for imine activation, while simultaneously locking this substrate in place as a result of favorable dispersion interactions between the quinolinium moiety and the neighboring phenyl ring - a stereochemical determining scenario reminiscent to the observed $s y n-(2 S, 3 R)$ product using the $C_{1}$-symmetric catalyst. Comprising this two-point hydrogen bond contact was homo/heteronuclear hydrogen bonds measuring $1.88 \AA$ and $1.75 \AA$. Interestingly, a bifurcated hydrogen bond to the carbonyl oxygen atom of the aryl ester played a key role in stabilizing this transition state, having bond distances of $1.71 \AA$ and $1.92 \AA$. A further heteronuclear stabilizing hydrogen bond contact was found to exist with the nitronate oxygen atom, measuring $2.14 \AA$. Meanwhile, both amidine arms of catalyst were pointed in the same direction, somewhat like a crown, while the large tert-butyl of the $N$-Boc group and large diisopropyl ester of the nitronate were oriented away from the catalyst backbone, thus, minimizing steric interactions. This favorable hydrogen bond network in conjunction with dispersion interactions and minimization of steric strain helped govern stereoinduction.
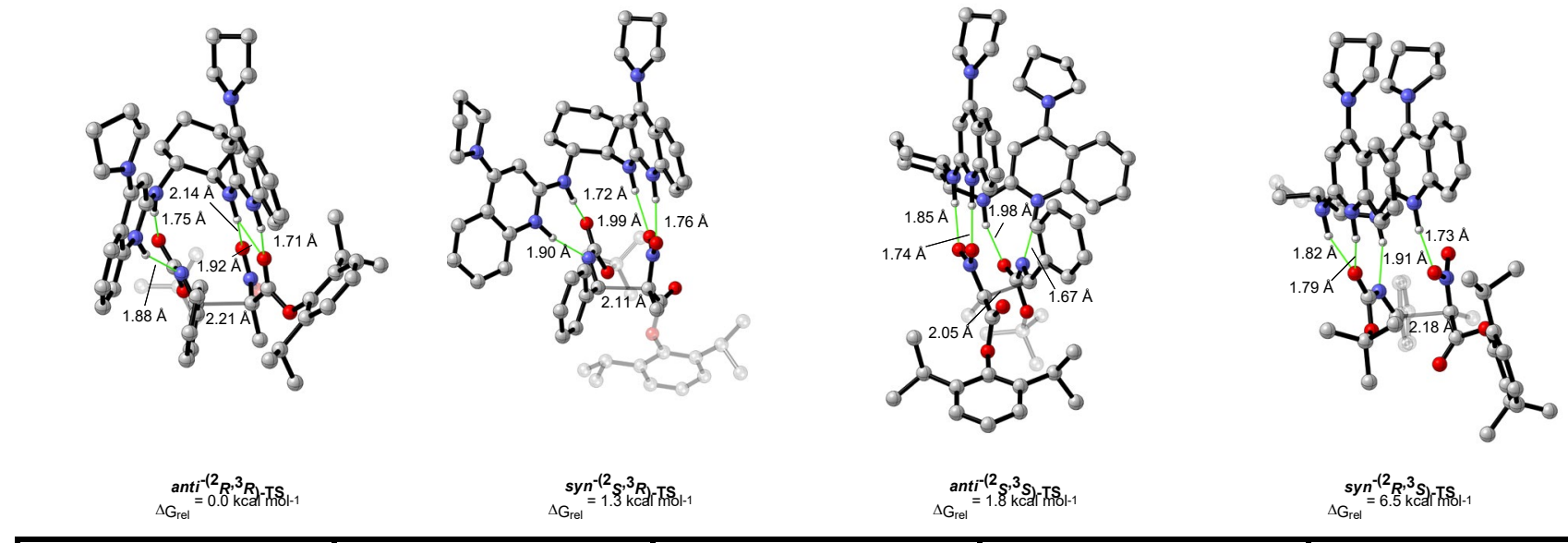

\begin{tabular}{|c|c|c|c|c|}
\hline Structure & $\begin{array}{c}\text { Nitro Group } \\
\text { Heteronuclear } \\
\text { Hydrogen Bond } \\
\text { Distances } \\
(\AA, N-\mathrm{H}-\mathrm{O})\end{array}$ & $\begin{array}{c}\text { Imine Group } \\
\text { Homonuclear } \\
\text { Hydrogen Bond } \\
\text { Distances } \\
(\AA ̊ N-\mathrm{H}-\mathrm{N})\end{array}$ & $\begin{array}{l}\text { Imine Group } \\
\text { Heteronuclear } \\
\text { Hydrogen Bond } \\
\text { Distances } \\
(\AA, \mathrm{N}-\mathrm{H}-\mathrm{O})\end{array}$ & $\begin{array}{c}\text { Transition State C-C } \\
\text { Bond Forming } \\
\text { Distances } \\
(\AA)\end{array}$ \\
\hline anti-(2R,3R)-TS $($ eq) & $1.71,1.92,2.14$ & 1.88 & 1.75 & 2.21 \\
\hline syn- $(2 S, 3 R)-\mathrm{TS}_{(\mathrm{eq})}$ & $1.76,1.99$ & 1.90 & 1.72 & 2.11 \\
\hline anti-(2S,3S)-TS $($ eq) & $1.74,1.85$ & 1.67 & 1.98 & 2.05 \\
\hline syn- $(2 R, 3 S)-\mathrm{TS}_{(\mathrm{eq})}$ & 1.73 & 1.91 & $1.79,1.82$ & 2.18 \\
\hline
\end{tabular}


Figure S1. Transition state geometries computed at the M06-2X/6-31G(d) level, providing reported free energies relative to anti-(2R,3R)-TS. Bonds in green represent the various homo- and heteronuclear hydrogen bonding interactions. Bond distances are reported in Ångstroms.

Optimized Transition State- and Precomplex Structures Using the $\mathrm{C}_{2-}$ Symmetric catalyst.

Table S3. Single point energies of all structures calculated at the $\mathrm{SMD}_{\text {(Toluene) }} \mathrm{M} 06-2 \mathrm{X} / 6-$ $311++\mathrm{G}(2 \mathrm{~d}, 2 \mathrm{p})$ level of theory and thermal correction to Gibbs Free Energy and Gibbs Free Energy calculated at the M06-2X/6-31G(d) level of theory. All energies are reported in Hartrees.

\begin{tabular}{|c|c|c|c|c|}
\hline Structure & $\begin{array}{c}\text { Single Point } \\
\text { Energies (E) } \\
\text { SMD }_{\text {(Toluene) M06- }} \text { 2X/6- } \\
\text { 2X/6) } \\
\text { 311++G(2d,2p) }\end{array}$ & $\begin{array}{c}\text { Thermal } \\
\text { Corrections to } \\
\text { Gibbs Free } \\
\text { Energies (G) } \\
\text { M06-2X/6-31G(d) }\end{array}$ & $\begin{array}{c}\text { Gibbs Free } \\
\text { Energies (G) } \\
\text { M06-2X/6- } \\
\text { 31G(d) }\end{array}$ & $\begin{array}{c}\text { Gibbs Free } \\
\text { Energies (G) } \\
\text { SMD }_{\text {(Toluene) M06 }} \\
-2 X / 6- \\
\text { 311++G(2d,2p)// } \\
\text { M06-2X/6- } \\
\text { 31G(d) } \\
\end{array}$ \\
\hline \multirow[t]{2}{*}{$\begin{array}{c}\mathrm{C} 2 \text { anti- } \\
(2 R, 3 R)-\mathrm{TS}\end{array}$} & & & & \\
\hline & -3182.376310 & 1.184436 & -3180.139794 & -3181.191874 \\
\hline \multirow[t]{2}{*}{$\begin{array}{c}\mathrm{C} 2 \text { anti- } \\
(2 S, 3 S)-\mathrm{TS}\end{array}$} & & & & \\
\hline & -3182.367690 & 1.178713 & -3180.129050 & -3181.188977 \\
\hline \multirow[t]{2}{*}{$\begin{array}{c}\text { C2 syn- } \\
(2 S, 3 R)-\mathrm{TS}\end{array}$} & & & & \\
\hline & -3182.371031 & 1.181168 & -3180.134395 & -3181.189863 \\
\hline \multirow[t]{2}{*}{$\begin{array}{c}\text { C2 syn- } \\
(2 R, 3 S)-\mathrm{TS}\end{array}$} & & & & \\
\hline & -3182.366929 & 1.185392 & -3180.128689 & -3181.181537 \\
\hline $\begin{array}{c}\text { C2 anti- } \\
(2 R, 3 R)-T S \\
\text { Precomplex }\end{array}$ & -3182.386712 & & -3180.150691 & -3181.205897 \\
\hline
\end{tabular}




\begin{tabular}{|c|c|c|c|c|}
\hline & & 1.180815 & & \\
\hline $\begin{array}{c}\text { C2 } \text { anti- } \\
\text { (2S,3S)-TS } \\
\text { Precomplex }\end{array}$ & & & & \\
& -3182.385705 & 1.174029 & -3180.146142 & -3181.211676 \\
\hline $\begin{array}{c}\text { C2 syn- } \\
(2 S, 3 R)-T S 2\end{array}$ & & & & \\
Precomplex & & & & \\
\hline $\begin{array}{c}\text { C2 syn- } \\
\text { Pr,3S)-TS2 }\end{array}$ & -3182.38548 & 1.17684 & -3180.149718 & -3181.20864 \\
\hline Precomplex & & & & \\
\end{tabular}

Structure C2 anti-(2R,3R)-TS

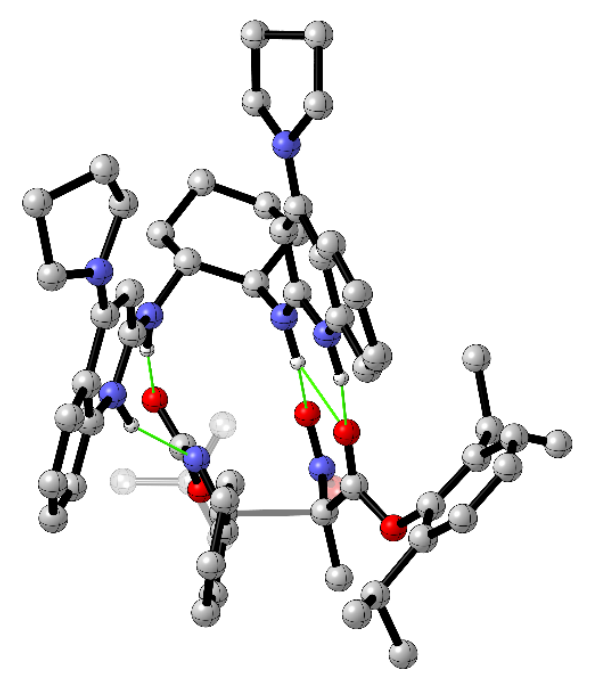

- Thermochemistry -

Zero-point correction= 1.286829 (Hartree/Particle) 
Thermal correction to Energy=

Thermal correction to Enthalpy=

Thermal correction to Gibbs Free Energy=

Sum of electronic and zero-point Energies=

Sum of electronic and thermal Energies=

Sum of electronic and thermal Enthalpies=

Sum of electronic and thermal Free Energies=
1.354432

1.355377

1.184436

$-3180.037401$

$-3179.969798$

$-3179.968853$

$-3180.139794$

Number of Imaginary Frequencies $=1$

E (Single Point Energy) [SMD(Toluene)M06-2X/6-311++G(2d,2p)] = -3182.376310

$\begin{array}{lrrr}\mathrm{O} & 1.65135800 & 0.90832300 & -1.10240300 \\ \mathrm{C} & 2.81916000 & 1.10895300 & -0.79594800 \\ \mathrm{O} & 3.30894400 & 2.36647100 & -0.70847400 \\ \mathrm{C} & 3.81132600 & 0.09774100 & -0.49507100 \\ \mathrm{C} & 5.20452100 & 0.52805800 & -0.14332000 \\ \mathrm{H} & 5.77960600 & -0.33709300 & 0.19568900 \\ \mathrm{H} & 5.73745500 & 0.97356000 & -0.98953800 \\ \mathrm{H} & 5.15449600 & 1.26710300 & 0.66231800 \\ \mathrm{~N} & 3.73959500 & -1.02527600 & -1.37654800 \\ \mathrm{O} & 2.63700100 & -1.50145600 & -1.67941500 \\ \mathrm{O} & 4.79535000 & -1.50283600 & -1.77976000 \\ \mathrm{C} & 3.01491300 & -0.82728600 & 1.35380400 \\ \mathrm{C} & 2.43871600 & 0.34028200 & 2.06983600 \\ \mathrm{C} & 3.17451000 & 0.95364600 & 3.08820600 \\ \mathrm{C} & 1.12163400 & 0.75914500 & 1.83003500 \\ \mathrm{C} & 2.58957500 & 1.93497600 & 3.88715400 \\ \mathrm{C} & 0.55108500 & 1.76234900 & 2.60437900 \\ \mathrm{C} & 1.27651600 & 2.33464300 & 3.65004600 \\ \mathrm{C} & 2.68142800 & -3.02702100 & 0.64785300 \\ \mathrm{O} & 1.93319100 & -3.98508000 & 0.45338100 \\ \mathrm{O} & 4.00126000 & -3.08220400 & 0.52540400 \\ \mathrm{C} & 4.67723300 & -4.29931300 & 0.05289100 \\ \mathrm{C} & 6.13680500 & -3.86598400 & -0.00575300 \\ \mathrm{H} & 6.47382600 & -3.53364600 & 0.98118000 \\ \mathrm{H} & 6.75883400 & -4.70973500 & -0.31702400 \\ \mathrm{H} & 6.26037700 & -3.04899700 & -0.72030700 \\ \mathrm{C} & 4.48463700 & -5.41389200 & 1.07524000 \\ \mathrm{H} & 3.43938000 & -5.71906300 & 1.13743300 \\ \mathrm{H} & 5.08591200 & -6.27803300 & 0.77671400 \\ \mathrm{H} & 4.82620700 & -5.08668400 & 2.06235000 \\ \mathrm{C} & 4.16619400 & -4.67740100 & -1.33420900 \\ \mathrm{H} & 4.16860800 & -3.79532700 & -1.97982000 \\ \mathrm{H} & 4.83684900 & -5.42883400 & -1.76246000\end{array}$




\begin{tabular}{|c|c|c|c|}
\hline $\mathrm{H}$ & 3.15903300 & -5.09404800 & -1.28661900 \\
\hline $\mathrm{N}$ & 2.17255500 & -1.80857600 & 1.05966500 \\
\hline $\mathrm{H}$ & 4.06331800 & -1.04703100 & 1.56718900 \\
\hline $\mathrm{H}$ & 0.81885700 & 3.09509400 & 4.27579800 \\
\hline $\mathrm{H}$ & 3.16025700 & 2.38524300 & 4.69376100 \\
\hline $\mathrm{H}$ & -0.47343700 & 2.07302900 & 2.41704800 \\
\hline $\mathrm{H}$ & 0.54996400 & 0.26281100 & 1.05079000 \\
\hline $\mathrm{H}$ & 4.19594800 & 0.63253000 & 3.27790500 \\
\hline $\mathrm{C}$ & -1.47031200 & -5.04979100 & -2.78925600 \\
\hline $\mathrm{C}$ & -0.58059400 & -4.22887700 & -3.72410200 \\
\hline $\mathrm{C}$ & -0.67848600 & -2.73746900 & -3.39712700 \\
\hline $\mathrm{C}$ & -0.33519600 & -2.48994400 & -1.92281400 \\
\hline $\mathrm{C}$ & -1.19620100 & -3.33074100 & -0.95156400 \\
\hline $\mathrm{C}$ & -1.09126300 & -4.81338300 & -1.32665800 \\
\hline $\mathrm{H}$ & 0.46154900 & -4.55472400 & -3.61131600 \\
\hline $\mathrm{H}$ & -0.85660900 & -4.39984400 & -4.76894300 \\
\hline $\mathrm{H}$ & -2.52215600 & -4.76815400 & -2.94576000 \\
\hline $\mathrm{H}$ & -1.39276300 & -6.11559100 & -3.02311200 \\
\hline $\mathrm{H}$ & 0.69604600 & -2.83030200 & -1.76534500 \\
\hline $\mathrm{H}$ & -2.24747000 & -3.03077300 & -0.99963200 \\
\hline $\mathrm{H}$ & -0.05537600 & -5.13540300 & -1.15267600 \\
\hline $\mathrm{H}$ & -1.72591300 & -5.39427400 & -0.65028200 \\
\hline $\mathrm{N}$ & -0.28018800 & -1.07406600 & -1.56328600 \\
\hline $\mathrm{N}$ & -0.70869700 & -3.13948000 & 0.40088000 \\
\hline $\mathrm{H}$ & 0.26427700 & -3.46973100 & 0.53189500 \\
\hline $\mathrm{C}$ & -1.19580400 & -2.32744100 & 1.34117100 \\
\hline $\mathrm{C}$ & -2.50926500 & -1.81574800 & 1.38260000 \\
\hline $\mathrm{C}$ & -0.72052900 & -1.28445500 & 3.44479700 \\
\hline $\mathrm{C}$ & -2.96536300 & -1.06816300 & 2.46594900 \\
\hline $\mathrm{H}$ & -3.18054000 & -2.09522200 & 0.58695900 \\
\hline $\mathrm{C}$ & 0.24991700 & -1.03400000 & 4.42900700 \\
\hline $\mathrm{C}$ & -2.01359900 & -0.72618000 & 3.52775800 \\
\hline $\mathrm{C}$ & -0.03536300 & -0.18297300 & 5.47183500 \\
\hline $\mathrm{H}$ & 1.22726100 & -1.49688600 & 4.32826400 \\
\hline $\mathrm{C}$ & -2.24904200 & 0.18262800 & 4.58421500 \\
\hline $\mathrm{C}$ & -1.28664700 & 0.44861300 & 5.53610400 \\
\hline $\mathrm{H}$ & 0.71891600 & 0.01920800 & 6.22507300 \\
\hline $\mathrm{H}$ & -3.18735800 & 0.71217200 & 4.64789800 \\
\hline $\mathrm{H}$ & -1.50140900 & 1.15181800 & 6.33326100 \\
\hline $\mathrm{N}$ & -0.35547000 & -2.05306800 & 2.36347800 \\
\hline $\mathrm{C}$ & -1.29004400 & -0.19931400 & -1.52879800 \\
\hline $\mathrm{C}$ & -2.64278000 & -0.50196100 & -1.81193400 \\
\hline $\mathrm{C}$ & -1.93772500 & 2.05831600 & -1.02459600 \\
\hline $\mathrm{C}$ & -3.64867900 & 0.45616800 & -1.73207400 \\
\hline $\mathrm{H}$ & -2.86614700 & -1.49393300 & -2.16124600 \\
\hline $\mathrm{C}$ & -3.30599800 & 1.78512100 & -1.22440500 \\
\hline
\end{tabular}




\begin{tabular}{|c|c|c|c|}
\hline $\mathrm{N}$ & -0.99226700 & 1.07991400 & -1.21077700 \\
\hline $\mathrm{C}$ & -1.49046000 & 3.31898100 & -0.59597100 \\
\hline $\mathrm{C}$ & -2.41047900 & 4.30159400 & -0.30741600 \\
\hline $\mathrm{C}$ & -3.78421800 & 4.03207200 & -0.41587400 \\
\hline $\mathrm{H}$ & -4.51018300 & 4.79139700 & -0.14693000 \\
\hline $\mathrm{C}$ & -4.21927900 & 2.80084200 & -0.86242000 \\
\hline $\mathrm{H}$ & -5.28281000 & 2.61898300 & -0.90729200 \\
\hline $\mathrm{C}$ & 0.90746500 & 5.57146900 & -1.80871700 \\
\hline $\mathrm{C}$ & 1.22277200 & 4.60062600 & -2.75029300 \\
\hline $\mathrm{C}$ & 2.03449200 & 3.50796600 & -2.42679100 \\
\hline $\mathrm{C}$ & 2.47372000 & 3.41966900 & -1.10144400 \\
\hline $\mathrm{C}$ & 2.20455600 & 4.39323800 & -0.13097900 \\
\hline $\mathrm{C}$ & 1.41027900 & 5.47624700 & -0.51337100 \\
\hline $\mathrm{H}$ & 0.28588500 & 6.41638700 & -2.08879900 \\
\hline $\mathrm{H}$ & 0.85419300 & 4.69983200 & -3.76768000 \\
\hline $\mathrm{C}$ & 2.46808200 & 2.54050600 & -3.51749100 \\
\hline $\mathrm{C}$ & 2.82448400 & 4.26724500 & 1.24687700 \\
\hline $\mathrm{H}$ & 1.18414100 & 6.25518400 & 0.20733900 \\
\hline $\mathrm{C}$ & 3.25141200 & 3.28998100 & -4.60442800 \\
\hline $\mathrm{H}$ & 3.15059600 & 1.80195800 & -3.08528600 \\
\hline $\mathrm{C}$ & 1.28217300 & 1.78661400 & -4.12916900 \\
\hline $\mathrm{H}$ & 1.62956300 & 1.13156500 & -4.93420200 \\
\hline $\mathrm{H}$ & 0.77789400 & 1.16847900 & -3.38165400 \\
\hline $\mathrm{H}$ & 0.55323900 & 2.48423500 & -4.55622600 \\
\hline $\mathrm{H}$ & 3.63432700 & 2.58346400 & -5.34694100 \\
\hline $\mathrm{H}$ & 2.61365000 & 4.01106900 & -5.12605700 \\
\hline $\mathrm{H}$ & 4.09762900 & 3.83478100 & -4.17719800 \\
\hline $\mathrm{H}$ & 2.71482800 & 3.22128800 & 1.55422200 \\
\hline $\mathrm{C}$ & 4.32777700 & 4.57808200 & 1.18860500 \\
\hline $\mathrm{C}$ & 2.14693200 & 5.15040400 & 2.29184800 \\
\hline $\mathrm{H}$ & 4.78149000 & 4.42383900 & 2.17349200 \\
\hline $\mathrm{H}$ & 4.49115000 & 5.62119700 & 0.89722600 \\
\hline $\mathrm{H}$ & 4.84158900 & 3.93647100 & 0.46834000 \\
\hline $\mathrm{H}$ & 2.32030500 & 6.21292000 & 2.08866600 \\
\hline $\mathrm{H}$ & 2.56124100 & 4.93670100 & 3.28167500 \\
\hline $\mathrm{H}$ & 1.06570000 & 4.97726400 & 2.33019100 \\
\hline $\mathrm{H}$ & -2.06529400 & 5.27650700 & 0.02210800 \\
\hline $\mathrm{H}$ & -0.42170000 & 3.49039700 & -0.48322200 \\
\hline $\mathrm{C}$ & -5.06279900 & -0.35320400 & 3.69857800 \\
\hline $\mathrm{C}$ & -5.14855100 & -0.95233300 & 1.35814200 \\
\hline $\mathrm{C}$ & -6.48746100 & -0.74177400 & 3.30356300 \\
\hline $\mathrm{H}$ & -5.02136600 & 0.72135700 & 3.91164500 \\
\hline $\mathrm{H}$ & -4.69185000 & -0.90085000 & 4.56778500 \\
\hline $\mathrm{C}$ & -6.50756000 & -0.42725700 & 1.80763500 \\
\hline $\mathrm{H}$ & -5.20073300 & -2.03614100 & 1.17284900 \\
\hline $\mathrm{H}$ & -4.76566500 & -0.46463100 & 0.45582000 \\
\hline
\end{tabular}




$\begin{array}{lrrr}\mathrm{H} & -6.64507200 & -1.81299900 & 3.46505200 \\ \mathrm{H} & -7.23514300 & -0.19268000 & 3.87866400 \\ \mathrm{H} & -7.33189300 & -0.90357400 & 1.27298000 \\ \mathrm{H} & -6.56743600 & 0.65471500 & 1.64694300 \\ \mathrm{C} & -5.99096400 & 1.02663700 & -2.51914200 \\ \mathrm{C} & -5.22658600 & -1.26854400 & -2.47541500 \\ \mathrm{C} & -6.82281700 & 0.17314600 & -3.47581200 \\ \mathrm{H} & -5.56907400 & 1.91759400 & -2.98925900 \\ \mathrm{H} & -6.60871700 & 1.33369300 & -1.66649600 \\ \mathrm{C} & -6.70143400 & -1.22073700 & -2.86021300 \\ \mathrm{H} & -5.00950800 & -1.95779300 & -1.64983100 \\ \mathrm{H} & -4.61101100 & -1.56392600 & -3.33772700 \\ \mathrm{H} & -7.85130900 & 0.52998100 & -3.55352100 \\ \mathrm{H} & -6.37679200 & 0.18439800 & -4.47544400 \\ \mathrm{H} & -7.32920600 & -1.29792100 & -1.96586900 \\ \mathrm{H} & -6.97504500 & -2.02636100 & -3.54328200 \\ \mathrm{~N} & -4.91755200 & 0.11231100 & -2.07863900 \\ \mathrm{~N} & -4.26533000 & -0.67667300 & 2.49884700 \\ \mathrm{H} & -1.69005700 & -2.37958400 & -3.62953400 \\ \mathrm{H} & 0.01491800 & -2.15285900 & -4.01011300 \\ \mathrm{H} & 0.65399100 & -2.12478700 & 2.12870700 \\ \mathrm{H} & -0.00461900 & 1.33265400 & -1.08862700 \\ \mathrm{H} & 0.66410700 & -0.71941700 & -1.36848000\end{array}$

\section{Structure C2 anti-(2S,3S)-TS}

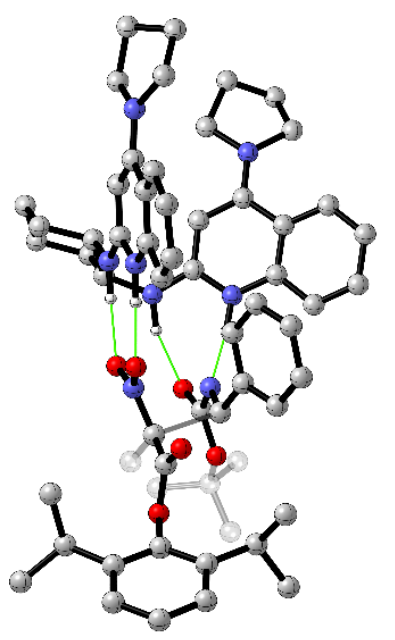


- Thermochemistry -

Zero-point correction=

Thermal correction to Energy=

Thermal correction to Enthalpy=

Thermal correction to Gibbs Free Energy=

Sum of electronic and zero-point Energies=

Sum of electronic and thermal Energies $=$

Sum of electronic and thermal Enthalpies=

Sum of electronic and thermal Free Energies=
1.285661 (Hartree/Particle)

1.353838

1.354782

1.178713

$-3180.022103$

$-3179.953926$

$-3179.952982$

$-3180.129050$

Number of Imaginary Frequencies $=1$

E (Single Point Energy) [SMD(Toluene)M06-2X/6-311++G(2d,2p)] = -3182.367690

$\begin{array}{llll}\mathrm{O} & 3.75332400 & 1.64760100 & -0.50039300 \\ \mathrm{C} & 3.92999500 & 0.67173500 & 0.17863800 \\ \mathrm{O} & 5.16333500 & 0.17356400 & 0.46829500 \\ \mathrm{C} & 2.89277400 & -0.22308400 & 0.78246100 \\ \mathrm{C} & 3.36014300 & -1.30443400 & 1.72550600 \\ \mathrm{H} & 2.52704300 & -1.94394500 & 2.01486700 \\ \mathrm{H} & 3.78448500 & -0.86261200 & 2.63425300 \\ \mathrm{H} & 4.13306500 & -1.90220400 & 1.24266300 \\ \mathrm{~N} & 1.72547100 & 0.42944400 & 1.21522400 \\ \mathrm{O} & 1.45587800 & 1.58098500 & 0.83895100 \\ \mathrm{O} & 0.92189400 & -0.22728000 & 1.90201700 \\ \mathrm{C} & 2.07107300 & -1.25347800 & -0.78214300 \\ \mathrm{C} & 1.55050900 & -0.19095200 & -1.67875300 \\ \mathrm{C} & 2.31701100 & 0.26328600 & -2.75177000 \\ \mathrm{C} & 0.26586600 & 0.33506000 & -1.48587000\end{array}$




\begin{tabular}{|c|c|c|c|}
\hline $\mathrm{C}$ & 1.81027000 & 1.21758400 & -3.62844000 \\
\hline $\mathrm{C}$ & -0.24400900 & 1.28265200 & -2.36425900 \\
\hline $\mathrm{C}$ & 0.52810300 & 1.72614900 & -3.43809500 \\
\hline $\mathrm{C}$ & 1.48313600 & -3.28573500 & 0.28352100 \\
\hline $\mathrm{O}$ & 0.68735300 & -3.88755900 & 1.00078800 \\
\hline $\mathrm{O}$ & 2.69293700 & -3.75233200 & -0.05407800 \\
\hline $\mathrm{C}$ & 3.18477100 & -5.03110100 & 0.45312500 \\
\hline $\mathrm{C}$ & 2.29712600 & -6.16416600 & -0.05193500 \\
\hline $\mathrm{H}$ & 1.30321900 & -6.11382900 & 0.39376800 \\
\hline $\mathrm{H}$ & 2.75451800 & -7.12392300 & 0.20691200 \\
\hline $\mathrm{H}$ & 2.20706000 & -6.11170100 & -1.14138500 \\
\hline $\mathrm{C}$ & 3.27313000 & -5.00357700 & 1.97608100 \\
\hline $\mathrm{H}$ & 3.90108900 & -4.17047400 & 2.30722700 \\
\hline $\mathrm{H}$ & 3.73518600 & -5.93272400 & 2.32340400 \\
\hline $\mathrm{H}$ & 2.28443000 & -4.91319300 & 2.42740500 \\
\hline $\mathrm{C}$ & 4.57605000 & -5.12158700 & -0.16262300 \\
\hline $\mathrm{H}$ & 4.51292100 & -5.10578600 & -1.25431900 \\
\hline $\mathrm{H}$ & 5.06201300 & -6.05108100 & 0.14624100 \\
\hline $\mathrm{H}$ & 5.19516100 & -4.28022300 & 0.16362900 \\
\hline $\mathrm{N}$ & 1.14474300 & -2.07841200 & -0.26683700 \\
\hline $\mathrm{H}$ & 3.05432600 & -1.65664300 & -1.04650300 \\
\hline $\mathrm{H}$ & 0.12983200 & 2.46815500 & -4.12327600 \\
\hline $\mathrm{H}$ & 2.41921500 & 1.56257500 & -4.45834800 \\
\hline $\mathrm{H}$ & -1.24619600 & 1.67515000 & -2.21440800 \\
\hline $\mathrm{H}$ & -0.32676800 & -0.01039300 & -0.64109900 \\
\hline $\mathrm{H}$ & 3.31444500 & -0.13936200 & -2.90397000 \\
\hline $\mathrm{C}$ & -3.34550600 & -2.13034200 & 4.94046600 \\
\hline $\mathrm{C}$ & -3.96291300 & -0.73200900 & 4.87351700 \\
\hline
\end{tabular}




\begin{tabular}{|c|c|c|c|}
\hline $\mathrm{C}$ & -3.03896000 & 0.23523700 & 4.13097600 \\
\hline $\mathrm{C}$ & -2.73514300 & -0.27114300 & 2.71477500 \\
\hline $\mathrm{C}$ & -2.10375900 & -1.67781300 & 2.78894600 \\
\hline $\mathrm{C}$ & -3.01868900 & -2.65548500 & 3.54017400 \\
\hline $\mathrm{H}$ & -2.08909500 & 0.33937900 & 4.67130700 \\
\hline $\mathrm{H}$ & -4.16778700 & -0.35396600 & 5.87937700 \\
\hline $\mathrm{H}$ & -4.92993200 & -0.78559300 & 4.35282600 \\
\hline $\mathrm{H}$ & -4.01969000 & -2.82475100 & 5.45047900 \\
\hline $\mathrm{H}$ & -2.42375400 & -2.08970900 & 5.53552800 \\
\hline $\mathrm{H}$ & -3.67611200 & -0.33191400 & 2.15249700 \\
\hline $\mathrm{H}$ & -1.18584600 & -1.56861100 & 3.38292100 \\
\hline $\mathrm{H}$ & -2.51911600 & -3.62751200 & 3.58875200 \\
\hline $\mathrm{H}$ & -3.95092100 & -2.80679000 & 2.98044800 \\
\hline $\mathrm{H}$ & -3.47940200 & 1.23704300 & 4.07083400 \\
\hline $\mathrm{N}$ & -1.83206500 & 0.60358100 & 1.98794900 \\
\hline $\mathrm{H}$ & -0.84013100 & 0.33900900 & 1.96711400 \\
\hline $\mathrm{N}$ & -1.63104300 & -2.16051700 & 1.49915600 \\
\hline $\mathrm{H}$ & -0.67583700 & -2.53258900 & 1.45674800 \\
\hline $\mathrm{C}$ & -2.29406100 & -2.15614600 & 0.32850400 \\
\hline $\mathrm{C}$ & -3.69344900 & -2.04881800 & 0.18750700 \\
\hline $\mathrm{C}$ & -2.05373500 & -2.22746400 & -2.04037100 \\
\hline $\mathrm{H}$ & -0.47832500 & -2.22573000 & -0.63890400 \\
\hline $\mathrm{C}$ & -4.30062200 & -2.10799900 & -1.06568600 \\
\hline $\mathrm{H}$ & -4.28804800 & -2.06418500 & 1.08454800 \\
\hline $\mathrm{C}$ & -1.15390400 & -2.23415600 & -3.12201800 \\
\hline $\mathrm{C}$ & -3.44258600 & -2.08493000 & -2.24769600 \\
\hline $\mathrm{C}$ & -1.62301000 & -2.04361900 & -4.40139500 \\
\hline $\mathrm{H}$ & -0.09428800 & -2.35431800 & -2.91639300 \\
\hline
\end{tabular}




\begin{tabular}{|c|c|c|c|}
\hline $\mathrm{C}$ & -3.87997000 & -1.85060000 & -3.57088300 \\
\hline $\mathrm{C}$ & -2.99308800 & -1.83037200 & -4.62578100 \\
\hline $\mathrm{H}$ & -0.92705600 & -2.03317800 & -5.23357800 \\
\hline $\mathrm{H}$ & -4.92176000 & -1.64365000 & -3.76869200 \\
\hline $\mathrm{H}$ & -3.35610900 & -1.63940400 & -5.62958400 \\
\hline $\mathrm{N}$ & -1.53534200 & -2.30046400 & -0.77114100 \\
\hline $\mathrm{C}$ & -2.17824500 & 1.73416700 & 1.35779000 \\
\hline C & -3.48527600 & 2.25206100 & 1.30434200 \\
\hline $\mathrm{C}$ & -1.38657200 & 3.57078200 & 0.04439200 \\
\hline $\mathrm{H}$ & -0.22644400 & 2.02043000 & 0.76924200 \\
\hline $\mathrm{C}$ & -3.78015800 & 3.40932700 & 0.57855200 \\
\hline $\mathrm{H}$ & -4.26307200 & 1.70135300 & 1.80838400 \\
\hline $\mathrm{C}$ & -2.66910900 & 4.15617800 & -0.01627800 \\
\hline $\mathrm{N}$ & -1.19470600 & 2.37909300 & 0.70320500 \\
\hline $\mathrm{C}$ & -0.26319400 & 4.18070400 & -0.54172700 \\
\hline $\mathrm{C}$ & -0.40000700 & 5.41588100 & -1.13397100 \\
\hline $\mathrm{C}$ & -1.64486600 & 6.06525700 & -1.12935400 \\
\hline $\mathrm{H}$ & -1.74009300 & 7.06012400 & -1.54993700 \\
\hline $\mathrm{C}$ & -2.75069300 & 5.44919000 & -0.58298400 \\
\hline $\mathrm{H}$ & -3.68092900 & 5.99586900 & -0.56386100 \\
\hline $\mathrm{C}$ & 8.51053600 & 2.50754200 & -0.28684100 \\
\hline $\mathrm{C}$ & 7.99762700 & 2.40231600 & 1.00335200 \\
\hline $\mathrm{C}$ & 6.86267800 & 1.63558700 & 1.26103400 \\
\hline $\mathrm{C}$ & 6.27050500 & 0.98222300 & 0.17322400 \\
\hline $\mathrm{C}$ & 6.76003300 & 1.05332700 & -1.12852700 \\
\hline $\mathrm{C}$ & 7.89831800 & 1.83830600 & -1.33688900 \\
\hline $\mathrm{H}$ & 9.39509800 & 3.10928200 & -0.46961300 \\
\hline $\mathrm{H}$ & 8.49280400 & 2.91918200 & 1.81881100 \\
\hline
\end{tabular}




\begin{tabular}{|c|c|c|c|}
\hline $\mathrm{C}$ & 6.25246900 & 1.51432500 & 2.64683700 \\
\hline $\mathrm{C}$ & 6.11005200 & 0.32144000 & -2.28638700 \\
\hline $\mathrm{H}$ & 8.30852500 & 1.92150800 & -2.33985500 \\
\hline $\mathrm{C}$ & 7.26365000 & 1.73811000 & 3.77209500 \\
\hline $\mathrm{H}$ & 5.86914900 & 0.49140000 & 2.74014800 \\
\hline $\mathrm{C}$ & 5.07035100 & 2.48397000 & 2.80311300 \\
\hline $\mathrm{H}$ & 4.59479300 & 2.35274800 & 3.78103000 \\
\hline $\mathrm{H}$ & 4.30921700 & 2.35119200 & 2.02803700 \\
\hline $\mathrm{H}$ & 5.42605600 & 3.51771300 & 2.73386500 \\
\hline $\mathrm{H}$ & 6.80388400 & 1.50407800 & 4.73682400 \\
\hline $\mathrm{H}$ & 7.59109300 & 2.78227100 & 3.81441000 \\
\hline $\mathrm{H}$ & 8.14833500 & 1.10624000 & 3.65078300 \\
\hline $\mathrm{H}$ & 5.28011600 & -0.27178800 & -1.88411500 \\
\hline $\mathrm{C}$ & 7.09075000 & -0.65067400 & -2.95223000 \\
\hline $\mathrm{C}$ & 5.54343300 & 1.31920500 & -3.30333100 \\
\hline $\mathrm{H}$ & 6.59033300 & -1.21228000 & -3.74784700 \\
\hline $\mathrm{H}$ & 7.49602700 & -1.36263600 & -2.22775200 \\
\hline $\mathrm{H}$ & 7.93147600 & -0.11389800 & -3.40363800 \\
\hline $\mathrm{H}$ & 4.80175300 & 1.97029600 & -2.83167900 \\
\hline $\mathrm{H}$ & 5.07495600 & 0.79093700 & -4.14276100 \\
\hline $\mathrm{H}$ & 6.34334500 & 1.94417300 & -3.71461900 \\
\hline $\mathrm{H}$ & 0.46371600 & 5.89561500 & -1.58204000 \\
\hline $\mathrm{H}$ & 0.69388300 & 3.66566400 & -0.50546500 \\
\hline $\mathrm{C}$ & -6.42679600 & -2.74450500 & -2.27497800 \\
\hline $\mathrm{C}$ & -6.47344600 & -2.14184500 & 0.06668600 \\
\hline $\mathrm{C}$ & -7.65572500 & -3.34982700 & -1.59812100 \\
\hline $\mathrm{H}$ & -6.73782300 & -1.95498400 & -2.96903400 \\
\hline $\mathrm{H}$ & -5.82766900 & -3.47649700 & -2.82075100 \\
\hline
\end{tabular}




\begin{tabular}{|c|c|c|c|}
\hline $\mathrm{C}$ & -7.89352900 & -2.38896000 & -0.43300600 \\
\hline $\mathrm{H}$ & -6.16407600 & -2.94438000 & 0.75223500 \\
\hline $\mathrm{H}$ & -6.34666300 & -1.18381400 & 0.58368800 \\
\hline $\mathrm{H}$ & -7.42337400 & -4.35071400 & -1.22041300 \\
\hline $\mathrm{H}$ & -8.50311600 & -3.42748200 & -2.28162200 \\
\hline $\mathrm{H}$ & -8.53807100 & -2.79844600 & 0.34659100 \\
\hline $\mathrm{H}$ & -8.33826000 & -1.45602400 & -0.79467400 \\
\hline $\mathrm{C}$ & -6.13529700 & 3.07641200 & 1.17036800 \\
\hline $\mathrm{C}$ & -5.64467900 & 4.68780900 & -0.56644800 \\
\hline $\mathrm{C}$ & -7.41685300 & 3.78414500 & 0.74126500 \\
\hline $\mathrm{H}$ & -6.14412300 & 2.02511800 & 0.84540600 \\
\hline $\mathrm{H}$ & -5.96325400 & 3.10050600 & 2.25111600 \\
\hline $\mathrm{C}$ & -7.08815300 & 4.20266900 & -0.69134500 \\
\hline $\mathrm{H}$ & -5.63239100 & 5.73217900 & -0.23404600 \\
\hline $\mathrm{H}$ & -5.07969600 & 4.60509300 & -1.49727500 \\
\hline $\mathrm{H}$ & -7.58846200 & 4.66679100 & 1.36530400 \\
\hline $\mathrm{H}$ & -8.29080500 & 3.13506400 & 0.81814900 \\
\hline $\mathrm{H}$ & -7.74741100 & 4.97922300 & -1.08314200 \\
\hline $\mathrm{H}$ & -7.13715900 & 3.33711200 & -1.36030600 \\
\hline $\mathrm{N}$ & -5.07010400 & 3.80614800 & 0.46860800 \\
\hline $\mathrm{N}$ & -5.65372900 & -2.17547600 & -1.15190100 \\
\hline
\end{tabular}




\section{Structure C2 syn-(2S,3R)-TS}

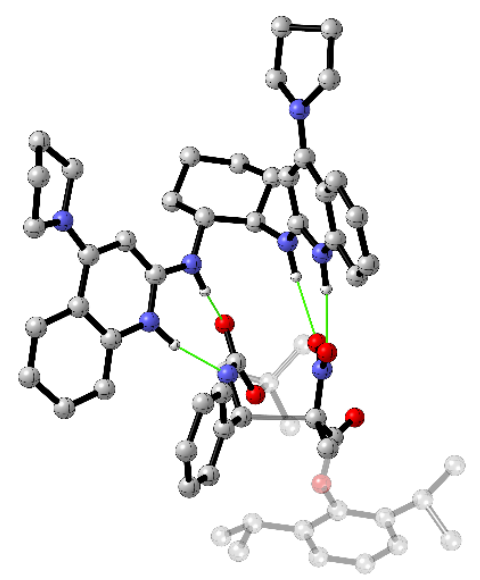

- Thermochemistry -

Zero-point correction $=$

Thermal correction to Energy=

Thermal correction to Enthalpy=

Thermal correction to Gibbs Free Energy=

Sum of electronic and zero-point Energies=

Sum of electronic and thermal Energies=

Sum of electronic and thermal Enthalpies=

Sum of electronic and thermal Free Energies=
1.286994 (Hartree/Particle)

1.354868

1.355812

1.181168

$-3180.028569$

$-3179.960695$

$-3179.959750$

$-3180.134395$

Number of Imaginary Frequencies $=1$

E (Single Point Energy) [SMD(Toluene)M06-2X/6-311++G(2d,2p)] = -3182.371031

$\begin{array}{lrrr}\mathrm{O} & 4.06151100 & -1.95815100 & 0.27952400 \\ \mathrm{C} & 3.94578900 & -1.05140900 & -0.49954300 \\ \mathrm{O} & 4.96010300 & -0.21644000 & -0.85593200 \\ \mathrm{C} & 2.74307900 & -0.67336600 & -1.30394200 \\ \mathrm{C} & 2.99122300 & -0.48740900 & -2.78234300 \\ \mathrm{H} & 3.79365100 & 0.23834500 & -2.92265200 \\ \mathrm{H} & 2.09395400 & -0.12989900 & -3.28584600 \\ \mathrm{H} & 3.29391500 & -1.43344900 & -3.24731900\end{array}$




\begin{tabular}{|c|c|c|c|}
\hline $\mathrm{N}$ & 1.58363500 & -1.38083700 & -0.98413900 \\
\hline $\mathrm{O}$ & 1.34958000 & -1.69356000 & 0.18735000 \\
\hline $\mathrm{O}$ & 0.71824200 & -1.54488100 & -1.87790800 \\
\hline $\mathrm{C}$ & 2.15871200 & 1.17142800 & -0.46058200 \\
\hline $\mathrm{C}$ & 1.66446100 & 1.88031900 & -1.66857200 \\
\hline $\mathrm{C}$ & 2.46650800 & 2.83310500 & -2.29888300 \\
\hline $\mathrm{C}$ & 0.37592200 & 1.62938300 & -2.15906900 \\
\hline $\mathrm{C}$ & 1.97862500 & 3.55274000 & -3.38818900 \\
\hline $\mathrm{C}$ & -0.10200800 & 2.33747900 & -3.25473900 \\
\hline $\mathrm{C}$ & 0.69431900 & 3.30694300 & -3.86585900 \\
\hline $\mathrm{C}$ & 1.60418400 & 0.49202100 & 1.72123800 \\
\hline $\mathrm{O}$ & 0.74502200 & 0.28576900 & 2.58620000 \\
\hline $\mathrm{O}$ & 2.90289400 & 0.27795600 & 1.92863100 \\
\hline $\mathrm{C}$ & 3.34961800 & -0.36211100 & 3.18048800 \\
\hline $\mathrm{C}$ & 2.73460400 & -1.75417200 & 3.29340000 \\
\hline $\mathrm{H}$ & 1.66469700 & -1.70353800 & 3.50100600 \\
\hline $\mathrm{H}$ & 3.22298100 & -2.28825500 & 4.11444900 \\
\hline $\mathrm{H}$ & 2.90310700 & -2.30499300 & 2.36436700 \\
\hline $\mathrm{C}$ & 3.02203700 & 0.53767300 & 4.36925000 \\
\hline $\mathrm{H}$ & 3.41851600 & 1.54360000 & 4.19607200 \\
\hline $\mathrm{H}$ & 3.50854500 & 0.13218700 & 5.26181300 \\
\hline $\mathrm{H}$ & 1.94944600 & 0.60079900 & 4.54845200 \\
\hline $\mathrm{C}$ & 4.85993600 & -0.47149600 & 3.01990300 \\
\hline $\mathrm{H}$ & 5.28079300 & -0.94071100 & 3.91405900 \\
\hline $\mathrm{H}$ & 5.30799700 & 0.52009800 & 2.91530000 \\
\hline $\mathrm{H}$ & 5.12070300 & -1.07930500 & 2.15115700 \\
\hline $\mathrm{N}$ & 1.23877300 & 0.97972800 & 0.49405300 \\
\hline $\mathrm{H}$ & 3.20162500 & 1.36471100 & -0.19511600 \\
\hline $\mathrm{H}$ & 0.31509100 & 3.86481400 & -4.71628700 \\
\hline $\mathrm{H}$ & 2.60425200 & 4.30078000 & -3.86481700 \\
\hline $\mathrm{H}$ & -1.09986200 & 2.13798700 & -3.63319300 \\
\hline $\mathrm{H}$ & -0.23163300 & 0.86898800 & -1.67743500 \\
\hline $\mathrm{H}$ & 3.46901700 & 3.02028000 & -1.92391100 \\
\hline $\mathrm{C}$ & -2.71225000 & -2.08562500 & 4.84333300 \\
\hline $\mathrm{C}$ & -3.55510800 & -0.81026800 & 4.77915600 \\
\hline $\mathrm{C}$ & -2.87030400 & 0.24471600 & 3.90918300 \\
\hline $\mathrm{C}$ & -2.61751300 & -0.27283200 & 2.48685700 \\
\hline $\mathrm{C}$ & -1.82729700 & -1.60007400 & 2.54069100 \\
\hline $\mathrm{C}$ & -2.48244400 & -2.65542500 & 3.44079300 \\
\hline $\mathrm{H}$ & -1.89727400 & 0.51126200 & 4.34282400 \\
\hline $\mathrm{H}$ & -3.72483100 & -0.41134500 & 5.78359100 \\
\hline $\mathrm{H}$ & -4.54464700 & -1.04818100 & 4.36194900 \\
\hline $\mathrm{H}$ & -3.19701500 & -2.83793500 & 5.47263700 \\
\hline $\mathrm{H}$ & -1.74461100 & -1.85661200 & 5.30826300 \\
\hline $\mathrm{H}$ & -3.57456800 & -0.43698600 & 1.97182800 \\
\hline $\mathrm{H}$ & -1.82995400 & -3.53335200 & 3.47154200 \\
\hline
\end{tabular}




\begin{tabular}{|c|c|c|c|}
\hline $\mathrm{H}$ & -3.44489500 & -2.99265700 & 3.04049000 \\
\hline $\mathrm{H}$ & -3.46004100 & 1.16733400 & 3.86133000 \\
\hline $\mathrm{N}$ & -1.81302600 & 0.64723000 & 1.70318900 \\
\hline $\mathrm{H}$ & -0.79393400 & 0.55466900 & 1.87252700 \\
\hline $\mathrm{N}$ & -1.45205300 & -2.06910500 & 1.20570700 \\
\hline $\mathrm{H}$ & -0.47858700 & -1.90222100 & 0.94032900 \\
\hline $\mathrm{C}$ & -2.28055000 & -2.37606300 & 0.19367400 \\
\hline $\mathrm{C}$ & -3.66455400 & -2.60392400 & 0.32832600 \\
\hline $\mathrm{C}$ & -2.45232000 & -2.81627400 & -2.15402100 \\
\hline $\mathrm{H}$ & -0.73007400 & -2.26163600 & -1.17061100 \\
\hline $\mathrm{C}$ & -4.44558400 & -3.03779000 & -0.74227900 \\
\hline $\mathrm{H}$ & -4.08783200 & -2.53125300 & 1.31284700 \\
\hline $\mathrm{C}$ & -1.78027200 & -2.83299800 & -3.38924400 \\
\hline $\mathrm{C}$ & -3.84074500 & -3.05237200 & -2.07277100 \\
\hline $\mathrm{C}$ & -2.49518700 & -3.03187600 & -4.54928100 \\
\hline $\mathrm{H}$ & -0.70787000 & -2.66633600 & -3.40487800 \\
\hline $\mathrm{C}$ & -4.54357700 & -3.19975100 & -3.28936900 \\
\hline $\mathrm{C}$ & -3.88841700 & -3.19269300 & -4.50227700 \\
\hline $\mathrm{H}$ & -1.97863400 & -3.04102800 & -5.50331300 \\
\hline $\mathrm{H}$ & -5.62006400 & -3.28536000 & -3.28697700 \\
\hline $\mathrm{H}$ & -4.45508200 & -3.30416300 & -5.41999600 \\
\hline $\mathrm{N}$ & -1.72051700 & -2.52690500 & -1.02266700 \\
\hline $\mathrm{C}$ & -2.22486900 & 1.77518100 & 1.11998100 \\
\hline $\mathrm{C}$ & -3.56258100 & 2.21412500 & 1.07608900 \\
\hline $\mathrm{C}$ & -1.52680300 & 3.71998700 & -0.07980000 \\
\hline $\mathrm{C}$ & -3.91989500 & 3.37524100 & 0.39262600 \\
\hline $\mathrm{H}$ & -4.30527500 & 1.59466800 & 1.55398500 \\
\hline $\mathrm{C}$ & -2.84525100 & 4.22578200 & -0.12362600 \\
\hline $\mathrm{N}$ & -1.26933900 & 2.49673900 & 0.49611800 \\
\hline $\mathrm{C}$ & -0.44845300 & 4.46235600 & -0.59054200 \\
\hline $\mathrm{C}$ & -0.66339900 & 5.72404500 & -1.09732500 \\
\hline $\mathrm{C}$ & -1.94974500 & 6.28309700 & -1.07210600 \\
\hline $\mathrm{C}$ & 8.79327100 & -0.76751500 & 0.64148400 \\
\hline $\mathrm{C}$ & 8.22651600 & -1.75124700 & -0.15437900 \\
\hline $\mathrm{C}$ & 6.93884500 & -1.60827500 & -0.68307900 \\
\hline $\mathrm{C}$ & 6.24637400 & -0.44462400 & -0.34784300 \\
\hline $\mathrm{C}$ & 6.79816700 & 0.58971900 & 0.42152400 \\
\hline $\mathrm{C}$ & 8.08743000 & 0.40167900 & 0.91452200 \\
\hline $\mathrm{H}$ & 9.79339900 & -0.90157400 & 1.04095400 \\
\hline $\mathrm{H}$ & 8.79236000 & -2.65056600 & -0.38187700 \\
\hline $\mathrm{C}$ & 6.38367200 & -2.67386200 & -1.61200300 \\
\hline $\mathrm{C}$ & 6.01283300 & 1.87033400 & 0.64719700 \\
\hline $\mathrm{H}$ & 8.54848700 & 1.17301800 & 1.52196100 \\
\hline $\mathrm{C}$ & 7.27624700 & -2.82914600 & -2.85013700 \\
\hline $\mathrm{H}$ & 5.39876600 & -2.35239000 & -1.96561800 \\
\hline $\mathrm{C}$ & 6.21321500 & -4.01231100 & -0.88584000 \\
\hline
\end{tabular}




\begin{tabular}{|c|c|c|c|}
\hline $\mathrm{H}$ & 5.78125400 & -4.75840800 & -1.56063800 \\
\hline $\mathrm{H}$ & 5.55986800 & -3.90221500 & -0.01771100 \\
\hline $\mathrm{H}$ & 7.18273700 & -4.39256800 & -0.54608600 \\
\hline $\mathrm{H}$ & 8.27324500 & -3.18879500 & -2.57586600 \\
\hline $\mathrm{H}$ & 7.39471900 & -1.87762300 & -3.37651900 \\
\hline $\mathrm{H}$ & 6.83973300 & -3.55657300 & -3.54194400 \\
\hline $\mathrm{H}$ & 4.99194700 & 1.57996300 & 0.93056600 \\
\hline $\mathrm{C}$ & 5.94298400 & 2.68021500 & -0.65633000 \\
\hline $\mathrm{C}$ & 6.57838100 & 2.74896900 & 1.76346000 \\
\hline $\mathrm{H}$ & 5.33905800 & 3.58434300 & -0.51437200 \\
\hline $\mathrm{H}$ & 5.51238200 & 2.09014800 & -1.47061100 \\
\hline $\mathrm{H}$ & 6.94818300 & 2.99137300 & -0.95999300 \\
\hline $\mathrm{H}$ & 7.55088500 & 3.16756600 & 1.48262800 \\
\hline $\mathrm{H}$ & 6.70697800 & 2.19800600 & 2.70018200 \\
\hline $\mathrm{H}$ & 5.90301800 & 3.58880400 & 1.95209100 \\
\hline $\mathrm{H}$ & -0.86545300 & -1.35086600 & 3.00396000 \\
\hline $\mathrm{H}$ & -0.31381500 & 2.07868000 & 0.45362100 \\
\hline $\mathrm{C}$ & -3.01267700 & 5.54857400 & -0.59082200 \\
\hline $\mathrm{H}$ & -3.98166900 & 6.02198500 & -0.54112400 \\
\hline $\mathrm{H}$ & -2.11086100 & 7.29747100 & -1.41967900 \\
\hline $\mathrm{H}$ & 0.17295700 & 6.29177400 & -1.49170000 \\
\hline $\mathrm{H}$ & 0.54628200 & 4.02768200 & -0.56850000 \\
\hline $\mathrm{C}$ & -6.26250500 & 2.85011500 & 0.87560700 \\
\hline $\mathrm{C}$ & -5.82734500 & 4.56393100 & -0.77514100 \\
\hline $\mathrm{C}$ & -7.57748100 & 3.46240300 & 0.40259700 \\
\hline $\mathrm{H}$ & -6.16385000 & 1.81568000 & 0.51444300 \\
\hline $\mathrm{H}$ & -6.14812500 & 2.84626400 & 1.96434900 \\
\hline $\mathrm{C}$ & -7.21376500 & 3.96091700 & -0.99569800 \\
\hline $\mathrm{H}$ & -5.92636100 & 5.59135900 & -0.40619400 \\
\hline $\mathrm{H}$ & -5.20937000 & 4.56992100 & -1.67545400 \\
\hline $\mathrm{H}$ & -7.85668700 & 4.30335700 & 1.04521600 \\
\hline $\mathrm{H}$ & -8.39448300 & 2.73885300 & 0.41037700 \\
\hline $\mathrm{H}$ & -7.91814300 & 4.69152400 & -1.39744000 \\
\hline $\mathrm{H}$ & -7.15149300 & 3.11955700 & -1.69375500 \\
\hline $\mathrm{C}$ & -6.53673300 & -4.32056000 & -1.35725200 \\
\hline $\mathrm{C}$ & -6.30104000 & -3.31794400 & 0.83211700 \\
\hline $\mathrm{C}$ & -7.44085400 & -5.03115000 & -0.35083100 \\
\hline $\mathrm{H}$ & -7.14728400 & -3.75137500 & -2.06787800 \\
\hline $\mathrm{H}$ & -5.89022200 & -5.00260200 & -1.91332600 \\
\hline $\mathrm{C}$ & -7.68536700 & -3.94371300 & 0.69496300 \\
\hline $\mathrm{H}$ & -5.68508500 & -3.89430900 & 1.53810300 \\
\hline $\mathrm{H}$ & -6.32141500 & -2.27530600 & 1.16722400 \\
\hline $\mathrm{H}$ & -6.91092600 & -5.87663100 & 0.09941800 \\
\hline $\mathrm{H}$ & -8.35476200 & -5.40516500 & -0.81564800 \\
\hline $\mathrm{H}$ & -8.05759900 & -4.32824700 & 1.64593500 \\
\hline $\mathrm{H}$ & -8.40059700 & -3.20602600 & 0.31702100 \\
\hline
\end{tabular}




\section{$\mathrm{N} \quad \begin{array}{llll}\mathrm{N} & -5.72968100 & -3.40925600 & -0.51949400\end{array}$ \\ $\mathrm{N} \quad-5.23048200 \quad 3.69410000 \quad 0.25781000$ \\ Structure C2 syn-(2R,3S)-TS}

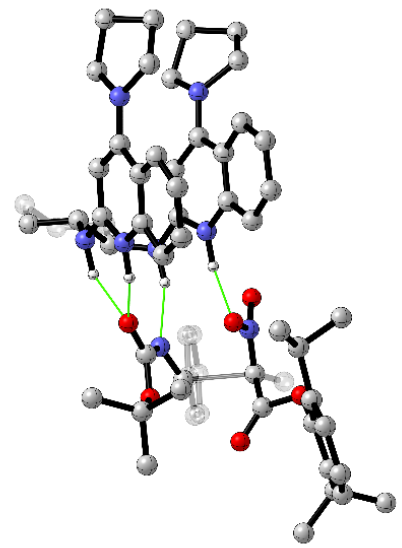

- Thermochemistry -

Zero-point correction $=$

Thermal correction to Energy=

Thermal correction to Enthalpy=

Thermal correction to Gibbs Free Energy=

Sum of electronic and zero-point Energies=

Sum of electronic and thermal Energies $=$

Sum of electronic and thermal Enthalpies=

Sum of electronic and thermal Free Energies=
1.286386 (Hartree/Particle)

1.353849

1.354793

1.185392

$-3180.027695$

$-3179.960233$

$-3179.959289$

$-3180.128689$

Number of Imaginary Frequencies $=1$

E (Single Point Energy) [SMD(Toluene)M06-2X/6-311++G(2d,2p)] = -3182.366929

$\begin{array}{llcl}\mathrm{O} & -4.93705700 & 0.40052500 & 0.41312100 \\ \mathrm{C} & -4.24313400 & 0.27488000 & -0.56118700 \\ \mathrm{O} & -4.27114800 & -0.80324200 & -1.38205800 \\ \mathrm{C} & -3.32820200 & 1.33297100 & -1.11497300 \\ \mathrm{C} & -3.91593600 & 2.16653300 & -2.21907100 \\ \mathrm{H} & -4.13987200 & 1.54078400 & -3.09165500 \\ \mathrm{H} & -3.22453800 & 2.95690800 & -2.51450100\end{array}$




\begin{tabular}{|c|c|c|c|}
\hline $\mathrm{H}$ & -4.84637600 & 2.62528200 & -1.87271700 \\
\hline $\mathrm{N}$ & -2.03256000 & 0.90120500 & -1.27820900 \\
\hline $\mathrm{O}$ & -1.22461100 & 1.54482200 & -1.95554500 \\
\hline $\mathrm{O}$ & -1.63582500 & -0.04373300 & -0.53119000 \\
\hline $\mathrm{C}$ & -2.77583800 & 2.58336300 & 0.57878900 \\
\hline $\mathrm{C}$ & -2.81016000 & 3.89904200 & -0.08715600 \\
\hline $\mathrm{C}$ & -1.68117400 & 4.44209600 & -0.71266900 \\
\hline $\mathrm{C}$ & -4.01290400 & 4.61245900 & -0.10252800 \\
\hline $\mathrm{C}$ & -1.76415100 & 5.67843300 & -1.34304600 \\
\hline $\mathrm{C}$ & -4.08979000 & 5.85480700 & -0.72292400 \\
\hline $\mathrm{C}$ & -2.96459900 & 6.38837700 & -1.34694600 \\
\hline $\mathrm{C}$ & -1.62061100 & 1.09270100 & 1.91761100 \\
\hline $\mathrm{O}$ & -0.56795100 & 0.53664900 & 2.28134800 \\
\hline $\mathrm{O}$ & -2.80774300 & 0.74094400 & 2.39851400 \\
\hline $\mathrm{C}$ & -3.01255000 & -0.46091000 & 3.19799400 \\
\hline $\mathrm{C}$ & -2.77998000 & -1.67444400 & 2.30455100 \\
\hline $\mathrm{H}$ & -1.79116700 & -1.62978200 & 1.83608000 \\
\hline $\mathrm{H}$ & -2.85437600 & -2.59124700 & 2.89967500 \\
\hline $\mathrm{C}$ & -4.48199600 & -0.36257000 & 3.59211400 \\
\hline $\mathrm{H}$ & -5.10328300 & -0.26792300 & 2.69901100 \\
\hline $\mathrm{H}$ & -4.64498500 & 0.50754800 & 4.23487300 \\
\hline $\mathrm{H}$ & -4.77690800 & -1.26153200 & 4.14110300 \\
\hline $\mathrm{C}$ & -2.13538500 & -0.46840600 & 4.44929000 \\
\hline $\mathrm{H}$ & -2.53520900 & -1.20622900 & 5.15163400 \\
\hline $\mathrm{H}$ & -2.16630900 & 0.51190800 & 4.93423900 \\
\hline $\mathrm{H}$ & -1.09696400 & -0.71696100 & 4.23049100 \\
\hline $\mathrm{N}$ & -1.60181900 & 2.14299300 & 1.05372400 \\
\hline $\mathrm{H}$ & -3.70065600 & 2.26838300 & 1.06388700 \\
\hline $\mathrm{H}$ & -3.02265300 & 7.35513500 & -1.83705500 \\
\hline $\mathrm{H}$ & -0.89045300 & 6.09155700 & -1.83826600 \\
\hline $\mathrm{H}$ & -5.02523600 & 6.40488400 & -0.72108800 \\
\hline $\mathrm{H}$ & -4.88933000 & 4.18651500 & 0.38064700 \\
\hline $\mathrm{H}$ & -0.75378100 & 3.87935600 & -0.72709800 \\
\hline $\mathrm{C}$ & 2.74909600 & 5.39880300 & 1.61161100 \\
\hline $\mathrm{C}$ & 3.72301600 & 4.67828600 & 2.54779100 \\
\hline $\mathrm{C}$ & 3.12694000 & 3.36986500 & 3.07491500 \\
\hline $\mathrm{C}$ & 2.71458000 & 2.45944300 & 1.91637700 \\
\hline $\mathrm{C}$ & 1.70912200 & 3.19800100 & 0.99719600 \\
\hline $\mathrm{C}$ & 2.31817300 & 4.49337300 & 0.45356000 \\
\hline $\mathrm{H}$ & 2.24246600 & 3.57966800 & 3.69068500 \\
\hline $\mathrm{H}$ & 3.99641700 & 5.32597100 & 3.38580800 \\
\hline $\mathrm{H}$ & 4.65263700 & 4.45842800 & 2.00362300 \\
\hline $\mathrm{H}$ & 3.20264600 & 6.31447900 & 1.22090500 \\
\hline $\mathrm{H}$ & 1.85901500 & 5.70304400 & 2.17755600 \\
\hline $\mathrm{H}$ & 3.60794800 & 2.21561300 & 1.33122500 \\
\hline $\mathrm{H}$ & 1.57830900 & 4.99039700 & -0.18173600 \\
\hline
\end{tabular}




\begin{tabular}{|c|c|c|c|}
\hline $\mathrm{H}$ & 3.18890500 & 4.27163400 & -0.17869000 \\
\hline $\mathrm{H}$ & 3.84040200 & 2.83765600 & 3.71279800 \\
\hline $\mathrm{N}$ & 2.10989000 & 1.21129500 & 2.37022400 \\
\hline $\mathrm{H}$ & 1.09156600 & 1.25434500 & 2.49972700 \\
\hline $\mathrm{N}$ & 1.10439500 & 2.31589500 & -0.00405500 \\
\hline $\mathrm{H}$ & 0.10151000 & 2.14039700 & 0.19923600 \\
\hline $\mathrm{C}$ & 1.76616500 & 1.31966700 & -0.61994400 \\
\hline $\mathrm{C}$ & 3.10444300 & 1.41882000 & -1.06787700 \\
\hline $\mathrm{C}$ & 1.69771900 & -0.95048600 & -1.34031000 \\
\hline $\mathrm{H}$ & 0.08052700 & 0.12696700 & -0.62017300 \\
\hline $\mathrm{C}$ & 3.73025500 & 0.36544600 & -1.72147900 \\
\hline $\mathrm{H}$ & 3.57543000 & 2.38547600 & -0.98790500 \\
\hline $\mathrm{C}$ & 0.95419200 & -2.14533600 & -1.35328300 \\
\hline $\mathrm{C}$ & 3.04326900 & -0.91963100 & -1.75880000 \\
\hline $\mathrm{C}$ & 1.56672900 & -3.32260800 & -1.71856200 \\
\hline $\mathrm{H}$ & -0.08842400 & -2.11019200 & -1.04496200 \\
\hline $\mathrm{C}$ & 3.64271500 & -2.15037700 & -2.10594600 \\
\hline $\mathrm{C}$ & 2.92579100 & -3.32862100 & -2.08046400 \\
\hline $\mathrm{H}$ & 1.00015900 & -4.24904700 & -1.71043000 \\
\hline $\mathrm{H}$ & 4.69108400 & -2.18015200 & -2.37277800 \\
\hline $\mathrm{H}$ & 3.41281000 & -4.26239000 & -2.34190700 \\
\hline $\mathrm{N}$ & 1.09903300 & 0.17497100 & -0.83523400 \\
\hline $\mathrm{C}$ & 2.55840100 & -0.02450700 & 2.07947500 \\
\hline $\mathrm{C}$ & 3.88890700 & -0.33340900 & 1.75778100 \\
\hline $\mathrm{C}$ & 1.97384100 & -2.33867900 & 1.86372300 \\
\hline $\mathrm{C}$ & 4.27889200 & -1.62494800 & 1.39396100 \\
\hline $\mathrm{H}$ & 4.59781900 & 0.47828600 & 1.75881800 \\
\hline $\mathrm{C}$ & 3.29714700 & -2.70408700 & 1.53307400 \\
\hline $\mathrm{N}$ & 1.64712300 & -1.02209900 & 2.09346200 \\
\hline $\mathrm{C}$ & 0.95437500 & -3.30002200 & 1.97217600 \\
\hline $\mathrm{C}$ & 1.24658300 & -4.63361000 & 1.79556400 \\
\hline $\mathrm{C}$ & 2.56804200 & -5.03158000 & 1.54469900 \\
\hline $\mathrm{H}$ & 2.81291700 & -6.08450100 & 1.46029700 \\
\hline $\mathrm{C}$ & 3.56667600 & -4.08770600 & 1.42551600 \\
\hline $\mathrm{H}$ & 4.57737100 & -4.43711900 & 1.28280000 \\
\hline $\mathrm{C}$ & -6.47304100 & -4.15568600 & -0.30275100 \\
\hline $\mathrm{C}$ & -7.13824400 & -2.98174000 & -0.62561200 \\
\hline $\mathrm{C}$ & -6.44006300 & -1.82714800 & -0.99577900 \\
\hline $\mathrm{C}$ & -5.04808100 & -1.91031600 & -1.00505200 \\
\hline $\mathrm{C}$ & -4.34030800 & -3.08684800 & -0.72279000 \\
\hline $\mathrm{C}$ & -5.08293100 & -4.21027800 & -0.36440600 \\
\hline $\mathrm{H}$ & -7.03732300 & -5.03743000 & -0.01625500 \\
\hline $\mathrm{H}$ & -8.22413000 & -2.95468900 & -0.59712600 \\
\hline $\mathrm{C}$ & -7.20059700 & -0.57984600 & -1.40943500 \\
\hline $\mathrm{C}$ & -2.83167000 & -3.12068800 & -0.89639500 \\
\hline $\mathrm{H}$ & -4.57387700 & -5.14029700 & -0.13236500 \\
\hline
\end{tabular}




\begin{tabular}{|c|c|c|c|}
\hline $\mathrm{C}$ & -8.07374100 & -0.86542900 & -2.63823300 \\
\hline $\mathrm{H}$ & -6.47692800 & 0.18910500 & -1.69773200 \\
\hline $\mathrm{C}$ & -8.04368100 & -0.03097900 & -0.25370500 \\
\hline $\mathrm{H}$ & -8.55261400 & 0.88895200 & -0.55891600 \\
\hline $\mathrm{H}$ & -7.41843400 & 0.18477300 & 0.61547100 \\
\hline $\mathrm{H}$ & -8.81271600 & -0.75287200 & 0.04196400 \\
\hline $\mathrm{H}$ & -8.57764900 & 0.04954000 & -2.96517600 \\
\hline $\mathrm{H}$ & -8.84608700 & -1.60691000 & -2.40893400 \\
\hline $\mathrm{H}$ & -7.47574000 & -1.24788200 & -3.47036100 \\
\hline $\mathrm{H}$ & -2.43947900 & -2.16079300 & -0.54438300 \\
\hline $\mathrm{C}$ & -2.49689000 & -3.23816000 & -2.39110100 \\
\hline $\mathrm{C}$ & -2.15126700 & -4.23713500 & -0.10336400 \\
\hline $\mathrm{H}$ & -1.41289000 & -3.27172900 & -2.55211000 \\
\hline $\mathrm{H}$ & -2.89657200 & -2.38575600 & -2.94679600 \\
\hline $\mathrm{H}$ & -2.92908200 & -4.15482600 & -2.80689200 \\
\hline $\mathrm{H}$ & -2.39284000 & -5.22672200 & -0.50689300 \\
\hline $\mathrm{H}$ & -2.44267600 & -4.21954100 & 0.95298500 \\
\hline $\mathrm{H}$ & -1.06132900 & -4.12183100 & -0.15769000 \\
\hline $\mathrm{H}$ & 0.85027400 & 3.48244500 & 1.62374800 \\
\hline $\mathrm{H}$ & 0.66248100 & -0.76060500 & 2.22500600 \\
\hline $\mathrm{H}$ & -3.53663500 & -1.71241700 & 1.51437300 \\
\hline $\mathrm{H}$ & -0.05546700 & -2.97457500 & 2.20775100 \\
\hline $\mathrm{H}$ & 0.45668600 & -5.37328200 & 1.87897300 \\
\hline $\mathrm{C}$ & 6.49118900 & -0.69807100 & 0.90207800 \\
\hline $\mathrm{C}$ & 6.07546700 & -2.98182800 & 0.22084200 \\
\hline $\mathrm{C}$ & 7.76977400 & -1.31207200 & 0.34158500 \\
\hline $\mathrm{H}$ & 6.09674900 & 0.07646600 & 0.22663100 \\
\hline $\mathrm{H}$ & 6.62458500 & -0.26146400 & 1.89652400 \\
\hline $\mathrm{C}$ & 7.22460600 & -2.39011900 & -0.59227000 \\
\hline $\mathrm{H}$ & 6.45756500 & -3.73928000 & 0.91609700 \\
\hline $\mathrm{H}$ & 5.29740200 & -3.42754000 & -0.40189200 \\
\hline $\mathrm{H}$ & 8.35693200 & -1.76639900 & 1.14579300 \\
\hline $\mathrm{H}$ & 8.39474200 & -0.57178900 & -0.16150800 \\
\hline $\mathrm{H}$ & 7.96029300 & -3.14352100 & -0.87901300 \\
\hline $\mathrm{H}$ & 6.83040200 & -1.92057000 & -1.50097400 \\
\hline $\mathrm{C}$ & 5.41276400 & -0.16064700 & -3.51441900 \\
\hline $\mathrm{C}$ & 5.64805800 & 1.82630400 & -2.15673900 \\
\hline $\mathrm{C}$ & 6.24913400 & 0.89243400 & -4.24250600 \\
\hline $\mathrm{H}$ & 6.04369500 & -1.02324500 & -3.27442500 \\
\hline $\mathrm{H}$ & 4.55207700 & -0.51131600 & -4.08901000 \\
\hline $\mathrm{C}$ & 6.84809900 & 1.69282200 & -3.08560800 \\
\hline $\mathrm{H}$ & 4.99149100 & 2.63635900 & -2.50605800 \\
\hline $\mathrm{H}$ & 5.90974300 & 2.02437800 & -1.11127300 \\
\hline $\mathrm{H}$ & 5.60440000 & 1.53631400 & -4.84912700 \\
\hline $\mathrm{H}$ & 6.99591700 & 0.43941400 & -4.89704400 \\
\hline $\mathrm{H}$ & 7.24920800 & 2.66241000 & -3.38487300 \\
\hline
\end{tabular}




$\begin{array}{rrrr}\mathrm{H} & 7.64649200 & 1.12217900 & -2.59785300 \\ \mathrm{~N} & 4.96660600 & 0.52785700 & -2.27739700 \\ \mathrm{~N} & 5.54349800 & -1.82159000 & 0.95634900\end{array}$

Structure C2 anti-(2R,3R)-TS Precomplex

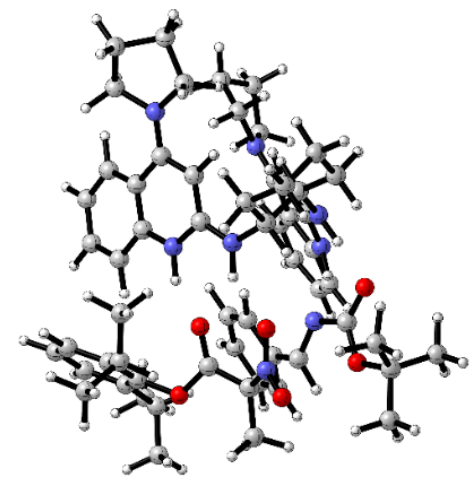

- Thermochemistry -

Zero-point correction=

Thermal correction to Energy=

Thermal correction to Enthalpy=

Thermal correction to Gibbs Free Energy=

Sum of electronic and zero-point Energies=

Sum of electronic and thermal Energies=

Sum of electronic and thermal Enthalpies=

Sum of electronic and thermal Free Energies=
1.286544 (Hartree/Particle)

1.355573

1.356517

1.180815

$-3180.044962$

$-3179.975933$

$-3179.974989$

$-3180.150691$

Number of Imaginary Frequencies $=0$

E (Single Point Energy) [SMD(Toluene)M06-2X/6-311++G(2d,2p)] = -3182.386712

$\begin{array}{llll}\mathrm{O} & 1.66251800 & 1.05210900 & -0.85070300 \\ \mathrm{C} & 2.85280800 & 1.38634700 & -0.90295600 \\ \mathrm{O} & 3.17482200 & 2.71731100 & -0.80327600 \\ \mathrm{C} & 3.99200900 & 0.56952300 & -1.07285300 \\ \mathrm{C} & 5.39404900 & 1.09714000 & -1.10956700 \\ \mathrm{H} & 6.06367900 & 0.43151100 & -0.55698900 \\ \mathrm{H} & 5.78040600 & 1.15418400 & -2.13327200 \\ \mathrm{H} & 5.43236400 & 2.09025000 & -0.66303900\end{array}$




\begin{tabular}{|c|c|c|c|}
\hline $\mathrm{N}$ & 3.83017600 & -0.73743000 & -1.50427600 \\
\hline $\mathrm{O}$ & 2.71187800 & -1.31074400 & -1.51094500 \\
\hline $\mathrm{O}$ & 4.84045600 & -1.33201300 & -1.90121800 \\
\hline $\mathrm{C}$ & 3.00437000 & -0.84093500 & 1.66320300 \\
\hline $\mathrm{C}$ & 2.33852500 & 0.29857600 & 2.30816500 \\
\hline $\mathrm{C}$ & 3.00198400 & 1.00313600 & 3.31825300 \\
\hline $\mathrm{C}$ & 1.01486800 & 0.62787500 & 1.98301700 \\
\hline $\mathrm{C}$ & 2.32542100 & 1.97911500 & 4.04437600 \\
\hline $\mathrm{C}$ & 0.35602200 & 1.63185700 & 2.68028100 \\
\hline $\mathrm{C}$ & 1.00261600 & 2.28686100 & 3.72947000 \\
\hline $\mathrm{C}$ & 2.93593800 & -2.97201500 & 0.69107400 \\
\hline $\mathrm{O}$ & 2.24651500 & -3.94318300 & 0.42181500 \\
\hline $\mathrm{O}$ & 4.23470900 & -2.88428000 & 0.55604200 \\
\hline $\mathrm{C}$ & 5.02598100 & -4.02091900 & 0.02921000 \\
\hline $\mathrm{C}$ & 6.42481900 & -3.42894100 & -0.06610300 \\
\hline $\mathrm{H}$ & 6.75692900 & -3.07700000 & 0.91554500 \\
\hline $\mathrm{H}$ & 7.12211100 & -4.19731900 & -0.41094000 \\
\hline $\mathrm{H}$ & 6.43083000 & -2.59453200 & -0.77041600 \\
\hline $\mathrm{C}$ & 4.97388800 & -5.15148600 & 1.04961700 \\
\hline $\mathrm{H}$ & 3.96163200 & -5.54735000 & 1.15241800 \\
\hline $\mathrm{H}$ & 5.62810600 & -5.96120200 & 0.71306100 \\
\hline $\mathrm{H}$ & 5.33206400 & -4.80697300 & 2.02469500 \\
\hline $\mathrm{C}$ & 4.50773500 & -4.44143200 & -1.34231700 \\
\hline $\mathrm{H}$ & 4.33240200 & -3.55265500 & -1.95242100 \\
\hline $\mathrm{H}$ & 5.27373700 & -5.05651000 & -1.82473800 \\
\hline $\mathrm{H}$ & 3.59049000 & -5.02645000 & -1.26247900 \\
\hline $\mathrm{N}$ & 2.30395900 & -1.84144800 & 1.27150400 \\
\hline $\mathrm{H}$ & 4.09553300 & -0.83409400 & 1.58271400 \\
\hline $\mathrm{H}$ & 0.47801600 & 3.05084400 & 4.29552200 \\
\hline $\mathrm{H}$ & 2.83100200 & 2.50436000 & 4.84859500 \\
\hline $\mathrm{H}$ & -0.66958600 & 1.88598700 & 2.42594200 \\
\hline $\mathrm{H}$ & 0.52579000 & 0.08348400 & 1.18029800 \\
\hline $\mathrm{H}$ & 4.03550500 & 0.76236800 & 3.55373100 \\
\hline $\mathrm{C}$ & -0.98257400 & -4.98184700 & -2.97467300 \\
\hline $\mathrm{C}$ & -0.09026700 & -4.05961700 & -3.80682400 \\
\hline $\mathrm{C}$ & -0.29048900 & -2.59870400 & -3.40003300 \\
\hline $\mathrm{C}$ & -0.04804900 & -2.42301500 & -1.89523600 \\
\hline $\mathrm{C}$ & -0.90563800 & -3.37104500 & -1.02530400 \\
\hline $\mathrm{C}$ & -0.69422500 & -4.81900300 & -1.48156100 \\
\hline $\mathrm{H}$ & 0.96083900 & -4.33555400 & -3.65206000 \\
\hline $\mathrm{H}$ & -0.29808100 & -4.18153100 & -4.87407500 \\
\hline $\mathrm{H}$ & -2.03857100 & -4.74491800 & -3.17207900 \\
\hline $\mathrm{H}$ & -0.83543300 & -6.02587300 & -3.26628300 \\
\hline $\mathrm{H}$ & 0.99251500 & -2.71114800 & -1.70519000 \\
\hline $\mathrm{H}$ & -1.96960600 & -3.13070600 & -1.10871600 \\
\hline $\mathrm{H}$ & 0.34908000 & -5.09388500 & -1.27369100 \\
\hline
\end{tabular}




\begin{tabular}{|c|c|c|c|}
\hline $\mathrm{H}$ & -1.32745300 & -5.47657600 & -0.87779200 \\
\hline $\mathrm{N}$ & -0.09756000 & -1.03186200 & -1.45068400 \\
\hline $\mathrm{N}$ & -0.50540000 & -3.24194400 & 0.36697300 \\
\hline $\mathrm{H}$ & 0.46868000 & -3.53110700 & 0.53305700 \\
\hline $\mathrm{C}$ & -1.08437300 & -2.50020600 & 1.31525600 \\
\hline $\mathrm{C}$ & -2.41129500 & -2.02783600 & 1.28770100 \\
\hline $\mathrm{C}$ & -0.77554300 & -1.50399400 & 3.47715000 \\
\hline $\mathrm{C}$ & -2.95872000 & -1.32844800 & 2.36278500 \\
\hline $\mathrm{H}$ & -3.01975900 & -2.29051700 & 0.43814200 \\
\hline $\mathrm{C}$ & 0.13034400 & -1.23067900 & 4.51466900 \\
\hline $\mathrm{C}$ & -2.07822300 & -0.96621100 & 3.47822500 \\
\hline $\mathrm{C}$ & -0.22750400 & -0.36986100 & 5.52736300 \\
\hline $\mathrm{H}$ & 1.11858200 & -1.68025400 & 4.48066500 \\
\hline $\mathrm{C}$ & -2.39014900 & -0.04971900 & 4.50704100 \\
\hline $\mathrm{C}$ & -1.48879700 & 0.24407100 & 5.50952100 \\
\hline $\mathrm{H}$ & 0.47833100 & -0.14696300 & 6.32053100 \\
\hline $\mathrm{H}$ & -3.33811700 & 0.46696600 & 4.50510500 \\
\hline $\mathrm{H}$ & -1.75892600 & 0.95553600 & 6.28205900 \\
\hline $\mathrm{N}$ & -0.33443400 & -2.26533400 & 2.41681700 \\
\hline $\mathrm{C}$ & -1.16241200 & -0.22522100 & -1.42268800 \\
\hline $\mathrm{C}$ & -2.47422500 & -0.59414400 & -1.80922800 \\
\hline $\mathrm{C}$ & -1.98872600 & 1.93775300 & -0.80115200 \\
\hline $\mathrm{C}$ & -3.54256200 & 0.29168800 & -1.73304700 \\
\hline $\mathrm{H}$ & -2.60708500 & -1.56786200 & -2.24581600 \\
\hline $\mathrm{C}$ & -3.32227700 & 1.59041900 & -1.09906900 \\
\hline $\mathrm{N}$ & -0.96672800 & 1.04383700 & -1.00342100 \\
\hline $\mathrm{C}$ & -1.65995300 & 3.18797200 & -0.25254700 \\
\hline $\mathrm{C}$ & -2.66396000 & 4.07652000 & 0.06063000 \\
\hline $\mathrm{C}$ & -4.00746500 & 3.72128200 & -0.14182300 \\
\hline $\mathrm{H}$ & -4.79989800 & 4.40346000 & 0.14583300 \\
\hline $\mathrm{C}$ & -4.32660200 & 2.50438400 & -0.70914000 \\
\hline $\mathrm{H}$ & -5.36974100 & 2.25032000 & -0.83011100 \\
\hline $\mathrm{C}$ & 0.30112000 & 5.63386400 & -1.46957600 \\
\hline $\mathrm{C}$ & 0.61511300 & 4.73712500 & -2.48254200 \\
\hline $\mathrm{C}$ & 1.57570600 & 3.73742600 & -2.29679600 \\
\hline $\mathrm{C}$ & 2.16758400 & 3.64678300 & -1.03083600 \\
\hline $\mathrm{C}$ & 1.87231500 & 4.53167700 & 0.01593100 \\
\hline $\mathrm{C}$ & 0.93612600 & 5.53793800 & -0.23382300 \\
\hline $\mathrm{H}$ & -0.43100500 & 6.41630100 & -1.64492700 \\
\hline $\mathrm{H}$ & 0.12988300 & 4.82956800 & -3.45056300 \\
\hline $\mathrm{C}$ & 2.00107000 & 2.86033100 & -3.46496100 \\
\hline $\mathrm{C}$ & 2.60218300 & 4.39566300 & 1.33932900 \\
\hline $\mathrm{H}$ & 0.69611900 & 6.25308900 & 0.54638700 \\
\hline $\mathrm{C}$ & 2.44382000 & 3.72597600 & -4.65263600 \\
\hline $\mathrm{H}$ & 2.87201400 & 2.27306300 & -3.15610100 \\
\hline $\mathrm{C}$ & 0.89843300 & 1.88328900 & -3.89207400 \\
\hline
\end{tabular}




\begin{tabular}{|c|c|c|c|}
\hline $\mathrm{H}$ & 1.19593300 & 1.35542200 & -4.80398300 \\
\hline $\mathrm{H}$ & 0.71484000 & 1.13797800 & -3.11405800 \\
\hline $\mathrm{H}$ & -0.03725600 & 2.41572300 & -4.10003400 \\
\hline $\mathrm{H}$ & 2.85630100 & 3.09321200 & -5.44423400 \\
\hline $\mathrm{H}$ & 1.60171400 & 4.28215100 & -5.07771900 \\
\hline $\mathrm{H}$ & 3.20891600 & 4.44725300 & -4.35310400 \\
\hline $\mathrm{H}$ & 2.61109500 & 3.32656800 & 1.59158900 \\
\hline $\mathrm{C}$ & 4.05943200 & 4.86222000 & 1.20529200 \\
\hline $\mathrm{C}$ & 1.91883100 & 5.15479900 & 2.47537100 \\
\hline $\mathrm{H}$ & 4.59265700 & 4.71668000 & 2.15069700 \\
\hline $\mathrm{H}$ & 4.09320700 & 5.92831400 & 0.95612000 \\
\hline $\mathrm{H}$ & 4.58494300 & 4.31019000 & 0.42306700 \\
\hline $\mathrm{H}$ & 1.99615900 & 6.23803900 & 2.33064100 \\
\hline $\mathrm{H}$ & 2.40429400 & 4.91783300 & 3.42709100 \\
\hline $\mathrm{H}$ & 0.85788500 & 4.89444600 & 2.55828700 \\
\hline $\mathrm{H}$ & -2.40802000 & 5.04442600 & 0.48024900 \\
\hline $\mathrm{H}$ & -0.61325100 & 3.42471600 & -0.07331400 \\
\hline $\mathrm{C}$ & -5.13218100 & -0.70753400 & 3.50544500 \\
\hline $\mathrm{C}$ & -5.09603000 & -1.31535300 & 1.16452600 \\
\hline $\mathrm{C}$ & -6.51781900 & -1.17298300 & 3.05915900 \\
\hline $\mathrm{H}$ & -5.15365500 & 0.36972200 & 3.70744300 \\
\hline $\mathrm{H}$ & -4.76687900 & -1.22746500 & 4.39370800 \\
\hline $\mathrm{C}$ & -6.49758200 & -0.86511500 & 1.56192600 \\
\hline $\mathrm{H}$ & -5.07849900 & -2.39920800 & 0.97525200 \\
\hline $\mathrm{H}$ & -4.70758300 & -0.80179200 & 0.28003500 \\
\hline $\mathrm{H}$ & -6.62442300 & -2.25035100 & 3.22132300 \\
\hline $\mathrm{H}$ & -7.31456500 & -0.66186800 & 3.60244100 \\
\hline $\mathrm{H}$ & -7.27351700 & -1.38703100 & 0.99847900 \\
\hline $\mathrm{H}$ & -6.60989000 & 0.21153400 & 1.39351100 \\
\hline $\mathrm{C}$ & -5.84345500 & 0.80046100 & -2.66404700 \\
\hline $\mathrm{C}$ & -4.95103600 & -1.44700100 & -2.73692900 \\
\hline $\mathrm{C}$ & -6.53323600 & -0.00824600 & -3.76217900 \\
\hline $\mathrm{H}$ & -5.43365300 & 1.74955300 & -3.01632600 \\
\hline $\mathrm{H}$ & -6.55359400 & 1.00097000 & -1.85272600 \\
\hline $\mathrm{C}$ & -6.38584000 & -1.44233700 & -3.25368700 \\
\hline $\mathrm{H}$ & -4.76628500 & -2.19592500 & -1.95664000 \\
\hline $\mathrm{H}$ & -4.24516200 & -1.63092600 & -3.56024600 \\
\hline $\mathrm{H}$ & -7.56979400 & 0.30063400 & -3.90834800 \\
\hline $\mathrm{H}$ & -5.99974500 & 0.11235700 & -4.71044300 \\
\hline $\mathrm{H}$ & -7.08590800 & -1.62902000 & -2.43220000 \\
\hline $\mathrm{H}$ & -6.54862500 & -2.19860900 & -4.02316000 \\
\hline $\mathrm{N}$ & -4.76236400 & -0.09301400 & -2.19810900 \\
\hline $\mathrm{N}$ & -4.27279900 & -1.00010700 & 2.34080100 \\
\hline $\mathrm{H}$ & -1.30278900 & -2.27777700 & -3.67815700 \\
\hline $\mathrm{H}$ & 0.40917800 & -1.94297500 & -3.92817600 \\
\hline $\mathrm{H}$ & 0.68126600 & -2.34669500 & 2.27884100 \\
\hline
\end{tabular}




$$
\begin{aligned}
& \mathrm{H} \quad 0.00379100 \quad 1.35501200 \quad-0.83665400 \\
& \mathrm{H} \quad 0.82499100 \quad-0.61524300 \quad-1.24947900
\end{aligned}
$$

\section{Structure C2 anti-(2S,3S)-TS Precomplex}

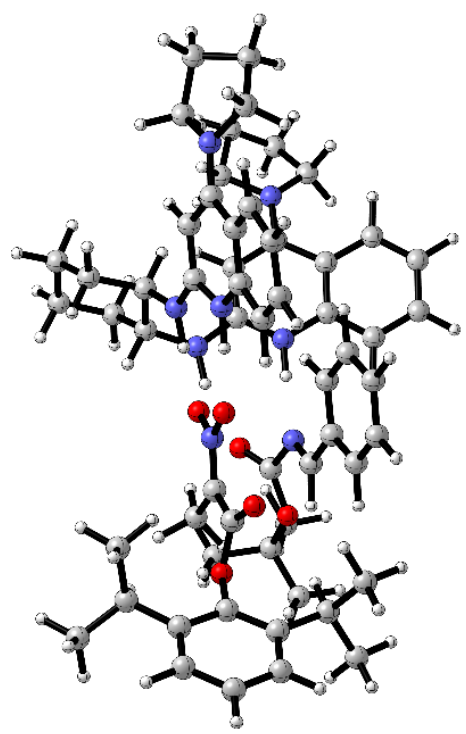

- Thermochemistry -

Zero-point correction=

Thermal correction to Energy=

Thermal correction to Enthalpy=

Thermal correction to Gibbs Free Energy=

Sum of electronic and zero-point Energies=

Sum of electronic and thermal Energies=

Sum of electronic and thermal Enthalpies=

Sum of electronic and thermal Free Energies=
1.284706 (Hartree/Particle)

1.354471

1.355415

1.174029

$-3180.035465$

$-3179.965700$

$-3179.964756$

$-3180.146142$

Number of Imaginary Frequencies $=0$

E (Single Point Energy) [SMD(Toluene)M06-2X/6-311++G(2d,2p)] = -3182.385705

$\mathrm{O}$ 


\begin{tabular}{|c|c|c|c|}
\hline$\sqrt{2}$ & 4.01867700 & 0.65505600 & 0.29920800 \\
\hline O & 5.19750700 & 0.06850800 & 0.69141500 \\
\hline $\mathrm{C}$ & 2.91723600 & -0.12783200 & 0.87138200 \\
\hline 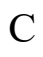 & 3.18689700 & -1.41664300 & 1.58761900 \\
\hline & 2.25196500 & -1.93039200 & 1.80968900 \\
\hline & 3.71982400 & -1.24217900 & 2.52920000 \\
\hline $\mathrm{H}$ & 3.82508600 & -2.06490700 & 0.97929100 \\
\hline & 1.67552300 & 0.36357200 & 0.83022900 \\
\hline $\mathrm{O}$ & 1.42676500 & 1.50085500 & 0.33546100 \\
\hline $\mathrm{O}$ & 0.69707300 & -0.33072100 & 1.29005800 \\
\hline $\mathrm{C}$ & 1.86909200 & -1.62653000 & -1.41084700 \\
\hline $\mathrm{C}$ & 1.54400900 & -0.40529000 & -2.13686100 \\
\hline $\mathrm{C}$ & 2.56302400 & 0.26115400 & -2.82150300 \\
\hline $\mathrm{C}$ & 0.24886600 & 0.13601000 & -2.11565500 \\
\hline $\mathrm{C}$ & 2.29053900 & 1.43234100 & -3.51876800 \\
\hline $\mathrm{C}$ & -0.02309300 & 1.29822100 & -2.81934300 \\
\hline $\mathrm{C}$ & 0.99603400 & 1.94307400 & -3.52616100 \\
\hline $\mathrm{C}$ & 1.31386400 & -3.47008800 & -0.08141000 \\
\hline $\mathrm{O}$ & 0.55594800 & -3.84202000 & 0.79191500 \\
\hline $\mathrm{O}$ & 2.46425400 & -4.03023600 & -0.42223300 \\
\hline $\mathrm{C}$ & 2.94064100 & -5.25708100 & 0.23696100 \\
\hline $\mathrm{C}$ & 1.95174700 & -6.38461900 & -0.03645400 \\
\hline $\mathrm{H}$ & 1.00060900 & -6.20951300 & 0.46868100 \\
\hline $\mathrm{H}$ & 2.37336000 & -7.32580200 & 0.32815400 \\
\hline $\mathrm{H}$ & 1.77872500 & -6.48252400 & -1.11244800 \\
\hline $\mathrm{C}$ & 3.14761900 & -5.01913100 & 1.72838700 \\
\hline $\mathrm{H}$ & 3.85378200 & -4.20090500 & 1.89502800 \\
\hline $\mathrm{H}$ & 3.57174800 & -5.92574800 & 2.17051900 \\
\hline $\mathrm{H}$ & 2.20644900 & -4.79362300 & 2.23120300 \\
\hline $\mathrm{C}$ & 4.26956100 & -5.51179900 & -0.46122400 \\
\hline $\mathrm{H}$ & 4.11932900 & -5.65302500 & -1.53505100 \\
\hline $\mathrm{H}$ & 4.73578300 & -6.41185800 & -0.05176100 \\
\hline $\mathrm{H}$ & 4.94920600 & -4.66801300 & -0.30926500 \\
\hline $\mathrm{N}$ & 0.96002900 & -2.34951200 & -0.85966700 \\
\hline $\mathrm{H}$ & 2.92462900 & -1.91240500 & -1.35492800 \\
\hline $\mathrm{H}$ & 0.77763200 & 2.85996100 & -4.06588900 \\
\hline $\mathrm{H}$ & 3.08882800 & 1.94509400 & -4.04513800 \\
\hline $\mathrm{H}$ & -1.02394600 & 1.71966800 & -2.80653800 \\
\hline $\mathrm{H}$ & -0.52249400 & -0.34094000 & -1.51801700 \\
\hline $\mathrm{H}$ & 3.57230500 & -0.14014100 & -2.80268600 \\
\hline $\mathrm{C}$ & -2.93967000 & -1.22506000 & 5.14861100 \\
\hline $\mathrm{C}$ & -3.56295900 & 0.15142000 & 4.90859300 \\
\hline $\mathrm{C}$ & -2.73057600 & 0.95618200 & 3.90901700 \\
\hline $\mathrm{C}$ & -2.59625000 & 0.21400200 & 2.57168200 \\
\hline $\mathrm{C}$ & -1.97866800 & -1.17978900 & 2.81975000 \\
\hline $\mathrm{C}$ & -2.78989600 & -1.99534100 & 3.83405000 \\
\hline
\end{tabular}




\begin{tabular}{|c|c|c|c|}
\hline $\mathrm{H}$ & -1.72172500 & 1.12305000 & 4.30825400 \\
\hline $\mathrm{H}$ & -3.65033100 & 0.70192900 & 5.84988400 \\
\hline $\mathrm{H}$ & -4.58391100 & 0.02631700 & 4.51971200 \\
\hline $\mathrm{H}$ & -3.54406800 & -1.80560400 & 5.85187800 \\
\hline $\mathrm{H}$ & -1.95123800 & -1.09834100 & 5.60917300 \\
\hline $\mathrm{H}$ & -3.59564900 & 0.09545100 & 2.12735300 \\
\hline $\mathrm{H}$ & -0.99124000 & -0.99558900 & 3.26291200 \\
\hline $\mathrm{H}$ & -2.28530700 & -2.95330700 & 3.99037500 \\
\hline $\mathrm{H}$ & -3.78748700 & -2.22428600 & 3.43863900 \\
\hline $\mathrm{H}$ & -3.16765700 & 1.94569100 & 3.73523100 \\
\hline $\mathrm{N}$ & -1.73905000 & 0.90440100 & 1.62871300 \\
\hline $\mathrm{H}$ & -0.77835900 & 0.52821200 & 1.50830900 \\
\hline $\mathrm{N}$ & -1.66554700 & -1.89405500 & 1.58239300 \\
\hline $\mathrm{H}$ & -0.67153200 & -2.03078800 & 1.39563200 \\
\hline $\mathrm{C}$ & -2.47880000 & -2.08205700 & 0.53248900 \\
\hline $\mathrm{C}$ & -3.88641300 & -2.04333600 & 0.59018700 \\
\hline $\mathrm{C}$ & -2.59343000 & -2.52399900 & -1.81470800 \\
\hline $\mathrm{H}$ & -0.84871900 & -2.35403200 & -0.70119900 \\
\hline $\mathrm{C}$ & -4.67162300 & -2.33224100 & -0.52455500 \\
\hline $\mathrm{H}$ & -4.33886000 & -1.90364200 & 1.55588000 \\
\hline $\mathrm{C}$ & -1.86680800 & -2.67165000 & -3.00917400 \\
\hline $\mathrm{C}$ & -4.00313700 & -2.47322300 & -1.81770300 \\
\hline $\mathrm{C}$ & -2.53513800 & -2.72079800 & -4.21242500 \\
\hline $\mathrm{H}$ & -0.78262800 & -2.71663900 & -2.96029300 \\
\hline $\mathrm{C}$ & -4.64737400 & -2.47931900 & -3.07429100 \\
\hline $\mathrm{C}$ & -3.93294600 & -2.60316500 & -4.24735800 \\
\hline $\mathrm{H}$ & -1.97451300 & -2.82554000 & -5.13530100 \\
\hline $\mathrm{H}$ & -5.71781700 & -2.34796000 & -3.13464100 \\
\hline $\mathrm{H}$ & -4.45470600 & -2.59843100 & -5.19779500 \\
\hline $\mathrm{N}$ & -1.88844500 & -2.37453400 & -0.64225400 \\
\hline $\mathrm{C}$ & -2.03723100 & 2.02021000 & 0.95412800 \\
\hline $\mathrm{C}$ & -3.29259700 & 2.66107600 & 0.96488900 \\
\hline $\mathrm{C}$ & -1.16488000 & 3.70896900 & -0.48440000 \\
\hline $\mathrm{H}$ & -0.11346300 & 2.04297000 & 0.20980900 \\
\hline $\mathrm{C}$ & -3.52652600 & 3.79901800 & 0.19185600 \\
\hline $\mathrm{H}$ & -4.08148600 & 2.20818000 & 1.54498700 \\
\hline $\mathrm{C}$ & -2.38777000 & 4.41433400 & -0.49368500 \\
\hline $\mathrm{N}$ & -1.05263500 & 2.51451700 & 0.18220100 \\
\hline $\mathrm{C}$ & -0.01367900 & 4.21563300 & -1.11559500 \\
\hline $\mathrm{C}$ & -0.06578000 & 5.44946600 & -1.72439100 \\
\hline $\mathrm{C}$ & -1.25024100 & 6.20340400 & -1.69177900 \\
\hline $\mathrm{H}$ & -1.27477600 & 7.19557500 & -2.12864900 \\
\hline $\mathrm{C}$ & -2.38062600 & 5.69849900 & -1.08483600 \\
\hline $\mathrm{H}$ & -3.25709900 & 6.32683700 & -1.03364600 \\
\hline $\mathrm{C}$ & 8.79249300 & 2.05369600 & 0.10505700 \\
\hline $\mathrm{C}$ & 8.16192800 & 2.08680500 & 1.34572100 \\
\hline
\end{tabular}




$\begin{array}{lrrr}\mathrm{C} & 6.93989900 & 1.44654600 & 1.54484600 \\ \mathrm{C} & 6.38110200 & 0.76946500 & 0.45353500 \\ \mathrm{C} & 6.98397200 & 0.71581100 & -0.80191700 \\ \mathrm{C} & 8.20624900 & 1.37650300 & -0.95515700 \\ \mathrm{H} & 9.74412900 & 2.55729000 & -0.03235200 \\ \mathrm{H} & 8.63045400 & 2.61608600 & 2.16934600 \\ \mathrm{C} & 6.19446500 & 1.50710800 & 2.86583400 \\ \mathrm{C} & 6.34980500 & -0.00841800 & -1.97338600 \\ \mathrm{H} & 8.70296300 & 1.35795900 & -1.92179800 \\ \mathrm{C} & 7.11990700 & 1.64003200 & 4.07625800 \\ \mathrm{H} & 5.63841300 & 0.56807200 & 2.96772900 \\ \mathrm{C} & 5.17944500 & 2.66088700 & 2.84262500 \\ \mathrm{H} & 4.59014800 & 2.67303700 & 3.76571900 \\ \mathrm{H} & 4.49486000 & 2.59137100 & 1.99221800 \\ \mathrm{H} & 5.70752600 & 3.61759000 & 2.76250100 \\ \mathrm{H} & 6.54128800 & 1.54214200 & 4.99977600 \\ \mathrm{H} & 7.60664200 & 2.62064800 & 4.10295100 \\ \mathrm{H} & 7.89877200 & 0.87172700 & 4.07482800 \\ \mathrm{H} & 5.44160700 & -0.50273300 & -1.60651500 \\ \mathrm{C} & 7.27432100 & -1.09413500 & -2.53499000 \\ \mathrm{C} & 5.94922900 & 0.99640500 & -3.06043900 \\ \mathrm{H} & 6.77759300 & -1.63920800 & -3.34463700 \\ \mathrm{H} & 7.56045200 & -1.81000600 & -1.75912100 \\ \mathrm{H} & 8.19134000 & -0.65794500 & -2.94429600 \\ \mathrm{H} & -5.25379200 & 1.73613700 & -2.65245600 \\ \mathrm{H} & -5.48030700 & 0.48640400 & -3.91127900 \\ \mathrm{H} & 6.83112700 & 1.52345400 & -3.43980200 \\ \mathrm{H} & 0.82229700 & 5.85192200 & -2.20077700 \\ \mathrm{H} & -5.90127700 & 3.62876500 & -1.07919000 \\ \mathrm{H} & -6.92263000 & -3.21094400 & -1.26904600 \\ \mathrm{C} & -6.63879500 & -2.24678800 & 0.93219800 \\ \mathrm{C} & -7.99984200 & -3.73460200 & -0.32032000 \\ \mathrm{C} & -7.37539400 & -2.54507900 & -2.01298000 \\ \mathrm{H} & -6.38062800 & -4.00414000 & -1.78832300 \\ \mathrm{H} & -8.10374900 & -2.60984000 & 0.70969400 \\ \mathrm{C} & -6.18412900 & -2.91839300 & 1.67495200 \\ \mathrm{H} & -6.48611900 & -1.21426300 & 1.26491000 \\ \mathrm{H} & -7.66106900 & -4.65774700 & 0.16075900 \\ \mathrm{H} & -8.93732300 & -3.94043700 & -0.83988200 \\ \mathrm{H} & -36905300 & 3.71028400 & 0.90560000 \\ \mathrm{H} & 5.10844800 & 4.49204600 & 0.48019500 \\ \mathrm{H} & 2.64600900 & 0.64626300 \\ \mathrm{H} & 3.78252800 & 1.97629000\end{array}$




$\begin{array}{llrc}\mathrm{C} & -6.80595100 & 4.79955400 & -0.98621500 \\ \mathrm{H} & -5.22094900 & 6.23262700 & -0.67214300 \\ \mathrm{H} & -4.80465900 & 4.99736600 & -1.88418700 \\ \mathrm{H} & -7.18595700 & 5.42054000 & 1.05464700 \\ \mathrm{H} & -8.02644100 & 3.92035000 & 0.62717300 \\ \mathrm{H} & -7.41891900 & 5.60180900 & -1.40096400 \\ \mathrm{H} & -6.94829100 & 3.90307200 & -1.59875200 \\ \mathrm{~N} & -4.78054500 & 4.31046400 & 0.12338700 \\ \mathrm{~N} & -6.01331500 & -2.45669700 & -0.38173700\end{array}$

\section{Structure C2 syn-(2S,3R)-TS Precomplex}

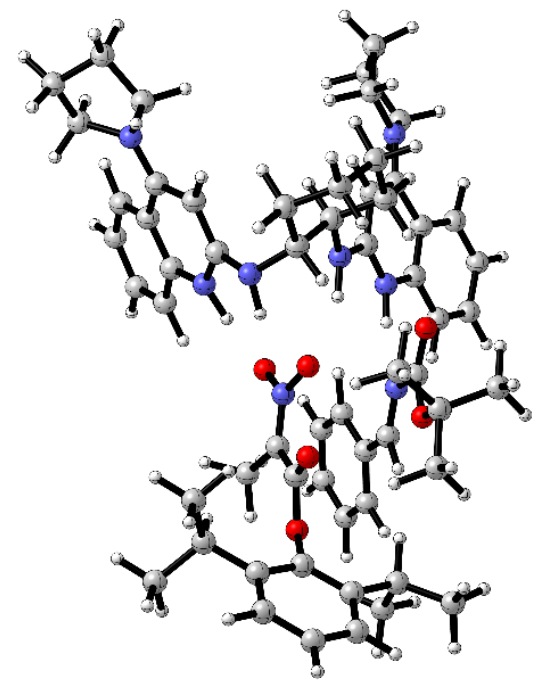

- Thermochemistry -

Zero-point correction=

Thermal correction to Energy=

Thermal correction to Enthalpy=

Thermal correction to Gibbs Free Energy=

Sum of electronic and zero-point Energies=

Sum of electronic and thermal Energies=

Sum of electronic and thermal Enthalpies=

Sum of electronic and thermal Free Energies=
1.286037 (Hartree/Particle)

1.355318

1.356262

1.176840

$-3180.040520$

$-3179.971239$

$-3179.970295$

$-3180.149718$ 
Number of Imaginary Frequencies $=0$

E (Single Point Energy) [SMD(Toluene)M06-2X/6-311++G(2d,2p)] = -3182.385480

$\begin{array}{lrrr}\mathrm{O} & 3.57493100 & -1.30945300 & 0.80190900 \\ \mathrm{C} & 3.64160100 & -0.93937200 & -0.34408400 \\ \mathrm{O} & 4.83895800 & -0.74373800 & -0.97656700 \\ \mathrm{C} & 2.53929600 & -0.67638500 & -1.28523800 \\ \mathrm{C} & 2.71662300 & -0.69375600 & -2.77391200 \\ \mathrm{H} & 3.77484400 & -0.63566600 & -3.02388700 \\ \mathrm{H} & 2.18626700 & 0.13855800 & -3.24269500 \\ \mathrm{H} & 2.30515400 & -1.61849400 & -3.19687700 \\ \mathrm{~N} & 1.30191700 & -0.57214100 & -0.81142600 \\ \mathrm{O} & 1.02820700 & -0.56961800 & 0.43832000 \\ \mathrm{O} & 0.32649400 & -0.42276300 & -1.63452400 \\ \mathrm{C} & 2.40635100 & 2.23915800 & -0.57376900 \\ \mathrm{C} & 2.26791500 & 2.52101600 & -2.00289400 \\ \mathrm{C} & 3.42033100 & 2.81022600 & -2.74089900 \\ \mathrm{C} & 1.02436600 & 2.46101300 & -2.64842600 \\ \mathrm{C} & 3.33431800 & 3.06388600 & -4.10509400 \\ \mathrm{C} & 0.94243800 & 2.71558300 & -4.01091800 \\ \mathrm{C} & 2.09417300 & 3.02122900 & -4.73847200 \\ \mathrm{C} & 1.57837100 & 1.82944700 & 1.57786200 \\ \mathrm{O} & 0.61733800 & 1.83171300 & 2.31710000 \\ \mathrm{O} & 2.82413800 & 1.53825100 & 1.90571400 \\ \mathrm{C} & 3.14234600 & 1.05719100 & 3.25981100 \\ \mathrm{C} & 2.32896500 & -0.19619800 & 3.57281000 \\ \mathrm{H} & 1.29420800 & 0.05718200 & 3.81518700 \\ \mathrm{H} & 2.77649400 & -0.69669600 & 4.43727800 \\ \mathrm{H} & 2.35726600 & -0.87388800 & 2.71467900 \\ \mathrm{C} & 2.89804700 & 2.18216400 & 4.25944300 \\ \mathrm{H} & 3.47811500 & 3.06841200 & 3.98441000 \\ \mathrm{H} & 3.22578800 & 1.85458000 & 5.25077800 \\ \mathrm{H} & 1.84029800 & 2.44521400 & 4.30979200 \\ \mathrm{C} & 4.62625000 & 0.73162500 & 3.16092500 \\ \mathrm{H} & 4.99537600 & 0.40146100 & 4.13623300 \\ \mathrm{H} & 5.18318000 & 1.62648800 & 2.86621600 \\ \mathrm{H} & 4.79806600 & -0.06246000 & 2.42811300 \\ \mathrm{~N} & 1.39508500 & 2.18219100 & 0.21231000 \\ \mathrm{H} & 3.42311300 & 2.08856800 & -0.19655800 \\ \mathrm{H} & 2.02474900 & 3.21565600 & -5.80420300 \\ \mathrm{H} & 4.23062500 & 3.29028600 & -4.67288900 \\ \mathrm{H} & -0.01835700 & 2.66351900 & -4.51277400 \\ \mathrm{H} & 0.14466800 & 2.16065200 & -2.08884900\end{array}$




\begin{tabular}{|c|c|c|c|}
\hline $\mathrm{H}$ & 4.38354100 & 2.83192200 & -2.23893400 \\
\hline $\mathrm{C}$ & -1.88396500 & -1.85354600 & 4.78270700 \\
\hline $\mathrm{C}$ & -2.72660800 & -0.57828800 & 4.70779900 \\
\hline $\mathrm{C}$ & -2.23524300 & 0.34622200 & 3.59187500 \\
\hline $\mathrm{C}$ & -2.21738200 & -0.37531800 & 2.23665800 \\
\hline $\mathrm{C}$ & -1.34888300 & -1.64047400 & 2.34428600 \\
\hline $\mathrm{C}$ & -1.87805900 & -2.57601900 & 3.43249700 \\
\hline $\mathrm{H}$ & -1.21360500 & 0.69285600 & 3.79440200 \\
\hline $\mathrm{H}$ & -2.70355100 & -0.04753700 & 5.66427300 \\
\hline $\mathrm{H}$ & -3.77588000 & -0.84993500 & 4.52314600 \\
\hline $\mathrm{H}$ & -2.26313400 & -2.52025200 & 5.56301000 \\
\hline $\mathrm{H}$ & -0.85289400 & -1.59392100 & 5.05697200 \\
\hline $\mathrm{H}$ & -3.23723200 & -0.68890500 & 1.96264200 \\
\hline $\mathrm{H}$ & -1.25496500 & -3.47473900 & 3.45914400 \\
\hline $\mathrm{H}$ & -2.90404900 & -2.89136400 & 3.20086300 \\
\hline $\mathrm{H}$ & -2.86252200 & 1.24183600 & 3.52605400 \\
\hline $\mathrm{N}$ & -1.68162100 & 0.46170800 & 1.16572400 \\
\hline $\mathrm{H}$ & -0.68614300 & 0.33745000 & 0.94695800 \\
\hline $\mathrm{N}$ & -1.10050800 & -2.26090000 & 1.03864700 \\
\hline $\mathrm{H}$ & -0.22215700 & -1.88927400 & 0.66013100 \\
\hline $\mathrm{C}$ & -2.04935100 & -2.46729600 & 0.09211900 \\
\hline $\mathrm{C}$ & -3.29293200 & -3.06964500 & 0.33857900 \\
\hline $\mathrm{C}$ & -2.56753600 & -2.32018900 & -2.23044700 \\
\hline $\mathrm{H}$ & -0.87496700 & -1.52896100 & -1.32059600 \\
\hline $\mathrm{C}$ & -4.17046800 & -3.38314900 & -0.70307000 \\
\hline $\mathrm{H}$ & -3.47726400 & -3.41831900 & 1.34081400 \\
\hline $\mathrm{C}$ & -2.14871300 & -1.85104700 & -3.48861200 \\
\hline $\mathrm{C}$ & -3.83770300 & -2.90852200 & -2.04612800 \\
\hline $\mathrm{C}$ & -3.00860200 & -1.92384100 & -4.56046500 \\
\hline $\mathrm{H}$ & -1.15494600 & -1.41983300 & -3.57455600 \\
\hline $\mathrm{C}$ & -4.70579900 & -2.91979600 & -3.16188300 \\
\hline $\mathrm{C}$ & -4.30276200 & -2.44247700 & -4.39075300 \\
\hline $\mathrm{H}$ & -2.69186800 & -1.56060500 & -5.53253300 \\
\hline $\mathrm{H}$ & -5.72039900 & -3.27426200 & -3.05764300 \\
\hline $\mathrm{H}$ & -4.99396700 & -2.46150200 & -5.22607300 \\
\hline $\mathrm{N}$ & -1.71622200 & -2.13957100 & -1.16704400 \\
\hline $\mathrm{C}$ & -2.23845400 & 1.62339200 & 0.79255500 \\
\hline $\mathrm{C}$ & -3.60042200 & 1.92695700 & 0.99356400 \\
\hline $\mathrm{C}$ & -1.91518600 & 3.74990400 & -0.24073300 \\
\hline $\mathrm{C}$ & -4.16346000 & 3.10804000 & 0.51617100 \\
\hline $\mathrm{H}$ & -4.21179800 & 1.17048600 & 1.45939400 \\
\hline $\mathrm{C}$ & -3.26477700 & 4.11583500 & -0.04780000 \\
\hline $\mathrm{N}$ & -1.46131500 & 2.50591200 & 0.13846600 \\
\hline $\mathrm{C}$ & -0.98445700 & 4.65907700 & -0.77374800 \\
\hline $\mathrm{C}$ & -1.37926100 & 5.94223200 & -1.08032500 \\
\hline $\mathrm{C}$ & -2.69911800 & 6.34953400 & -0.83697500 \\
\hline
\end{tabular}




\begin{tabular}{|c|c|c|c|}
\hline $\mathrm{C}$ & 8.29423100 & -2.12680000 & 0.85928700 \\
\hline $\mathrm{C}$ & 7.39546700 & -3.03225700 & 0.31306100 \\
\hline $\mathrm{C}$ & 6.21537300 & -2.59985200 & -0.29821100 \\
\hline $\mathrm{C}$ & 5.98034900 & -1.22458800 & -0.32574300 \\
\hline $\mathrm{C}$ & 6.86063000 & -0.28125100 & 0.21535400 \\
\hline $\mathrm{C}$ & 8.02876000 & -0.76138000 & 0.80700200 \\
\hline $\mathrm{H}$ & 9.20624700 & -2.48253400 & 1.32787300 \\
\hline $\mathrm{H}$ & 7.60927000 & -4.09669700 & 0.35956100 \\
\hline $\mathrm{C}$ & 5.24285100 & -3.60445400 & -0.88933900 \\
\hline $\mathrm{C}$ & 6.54395100 & 1.19985100 & 0.11098900 \\
\hline $\mathrm{H}$ & 8.73894600 & -0.06420000 & 1.23865700 \\
\hline $\mathrm{C}$ & 5.92920400 & -4.50942100 & -1.91904700 \\
\hline $\mathrm{H}$ & 4.45660800 & -3.05313600 & -1.41773100 \\
\hline $\mathrm{C}$ & 4.58212700 & -4.43402100 & 0.21835100 \\
\hline $\mathrm{H}$ & 3.85560600 & -5.13297900 & -0.20937100 \\
\hline $\mathrm{H}$ & 4.07115200 & -3.78663800 & 0.93541300 \\
\hline $\mathrm{H}$ & 5.33421400 & -5.02021600 & 0.75784500 \\
\hline $\mathrm{H}$ & 6.69852200 & -5.13117000 & -1.44979800 \\
\hline $\mathrm{H}$ & 6.40560400 & -3.92225300 & -2.70920000 \\
\hline $\mathrm{H}$ & 5.19774500 & -5.18123800 & -2.37930000 \\
\hline $\mathrm{H}$ & 5.48293200 & 1.32107500 & 0.37607500 \\
\hline $\mathrm{C}$ & 6.74372100 & 1.68411100 & -1.33261700 \\
\hline $\mathrm{C}$ & 7.36982300 & 2.06325800 & 1.06473600 \\
\hline $\mathrm{H}$ & 6.53143300 & 2.75716300 & -1.41132200 \\
\hline $\mathrm{H}$ & 6.09907000 & 1.14114600 & -2.02954100 \\
\hline $\mathrm{H}$ & 7.78385100 & 1.52843400 & -1.63798500 \\
\hline $\mathrm{H}$ & 8.41990200 & 2.09862200 & 0.75476500 \\
\hline $\mathrm{H}$ & 7.33281700 & 1.68825500 & 2.09170700 \\
\hline $\mathrm{H}$ & 6.99351600 & 3.09089000 & 1.05949300 \\
\hline $\mathrm{H}$ & -0.34878800 & -1.30949700 & 2.65821600 \\
\hline $\mathrm{H}$ & -0.43504000 & 2.33743100 & 0.09404200 \\
\hline $\mathrm{C}$ & -3.61600200 & 5.45516100 & -0.32641000 \\
\hline $\mathrm{H}$ & -4.60983600 & 5.81281500 & -0.10318200 \\
\hline $\mathrm{H}$ & -2.99871300 & 7.37281300 & -1.03386200 \\
\hline $\mathrm{H}$ & -0.65775600 & 6.64456000 & -1.48434100 \\
\hline $\mathrm{H}$ & 0.04401300 & 4.33821100 & -0.90797300 \\
\hline $\mathrm{C}$ & -6.34602400 & 2.29218800 & 1.27400800 \\
\hline $\mathrm{C}$ & -6.32899600 & 4.19360100 & -0.22138900 \\
\hline $\mathrm{C}$ & -7.76682500 & 2.81220200 & 1.07788900 \\
\hline $\mathrm{H}$ & -6.22178300 & 1.31473600 & 0.78522900 \\
\hline $\mathrm{H}$ & -6.05650200 & 2.19052400 & 2.32486900 \\
\hline $\mathrm{C}$ & -7.67662800 & 3.47720800 & -0.29546100 \\
\hline $\mathrm{H}$ & -6.45551900 & 5.16596600 & 0.26874100 \\
\hline $\mathrm{H}$ & -5.86593500 & 4.34796600 & -1.19844000 \\
\hline $\mathrm{H}$ & -8.00808800 & 3.55505600 & 1.84478700 \\
\hline $\mathrm{H}$ & -8.50799900 & 2.01276600 & 1.12899000 \\
\hline
\end{tabular}




$\begin{array}{lrrr}\mathrm{H} & -8.49592800 & 4.16802000 & -0.50255200 \\ \mathrm{H} & -7.65632900 & 2.71747800 & -1.08374800 \\ \mathrm{C} & -6.04426000 & -4.93603200 & -1.39510700 \\ \mathrm{C} & -5.59822100 & -4.50374200 & 0.94414300 \\ \mathrm{C} & -6.60043300 & -6.06811500 & -0.53234300 \\ \mathrm{H} & -6.87029500 & -4.36034700 & -1.82890800 \\ \mathrm{H} & -5.39510200 & -5.28586700 & -2.20073200 \\ \mathrm{C} & -6.84535000 & -5.37067700 & 0.80553000 \\ \mathrm{H} & -4.76855600 & -5.08843400 & 1.36605100 \\ \mathrm{H} & -5.74466700 & -3.61577300 & 1.56757500 \\ \mathrm{H} & -5.84947200 & -6.85611500 & -0.41607700 \\ \mathrm{H} & -7.49873700 & -6.50956700 & -0.96753700 \\ \mathrm{H} & -6.95995200 & -6.06083700 & 1.64291000 \\ \mathrm{H} & -7.74084100 & -4.74335800 & 0.74986300 \\ \mathrm{~N} & -5.28202800 & -4.10911900 & -0.43770900 \\ \mathrm{~N} & -5.50305100 & 3.29518800 & 0.60870200\end{array}$

\section{Structure C2 syn-(2R,3S)-TS Precomplex}

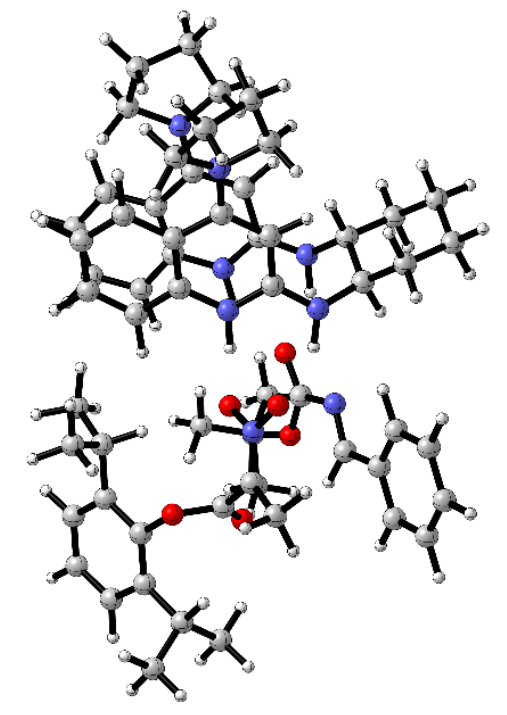

- Thermochemistry -

Zero-point correction=

Thermal correction to Energy=

1.285564 (Hartree/Particle)

Thermal correction to Enthalpy=

1.354404

Thermal correction to Gibbs Free Energy=

1.355349

Sum of electronic and zero-point Energies=

1.182657

Sum of electronic and thermal Energies=

$-3180.038004$

$-3179.969163$ 
Sum of electronic and thermal Enthalpies $=\quad-3179.968219$

Sum of electronic and thermal Free Energies $=\quad-3180.140911$

Number of Imaginary Frequencies $=0$

E (Single Point Energy) [SMD(Toluene)M06-2X/6-311++G(2d,2p)] = -3182.379347

\begin{tabular}{|c|c|c|c|}
\hline $\mathrm{O}$ & -4.68708000 & 0.33694400 & 0.18935400 \\
\hline $\mathrm{C}$ & -4.10429400 & 0.15838700 & -0.85164800 \\
\hline $\mathrm{O}$ & -4.24426200 & -0.95085900 & -1.61143400 \\
\hline $\mathrm{C}$ & -3.21403600 & 1.15691100 & -1.50740600 \\
\hline $\mathrm{C}$ & -3.73982600 & 2.14297800 & -2.49522300 \\
\hline $\mathrm{H}$ & -3.87610700 & 1.68249400 & -3.48138400 \\
\hline $\mathrm{H}$ & -3.03440400 & 2.97123200 & -2.59010800 \\
\hline $\mathrm{H}$ & -4.70881900 & 2.53301700 & -2.16669900 \\
\hline $\mathrm{N}$ & -1.93938200 & 1.06915000 & -1.22159600 \\
\hline $\mathrm{O}$ & -1.07343800 & 1.87848500 & -1.67098300 \\
\hline $\mathrm{O}$ & -1.52972400 & 0.14023400 & -0.38211600 \\
\hline $\mathrm{C}$ & -2.76149700 & 2.88044500 & 0.90240800 \\
\hline $\mathrm{C}$ & -2.85393400 & 4.06964400 & 0.06409400 \\
\hline $\mathrm{C}$ & -1.72264800 & 4.66899500 & -0.50510600 \\
\hline $\mathrm{C}$ & -4.12555900 & 4.59014100 & -0.20550000 \\
\hline $\mathrm{C}$ & -1.86856200 & 5.77748800 & -1.32660300 \\
\hline $\mathrm{C}$ & -4.26860100 & 5.70636500 & -1.02092600 \\
\hline $\mathrm{C}$ & -3.13877200 & 6.29814800 & -1.58208500 \\
\hline $\mathrm{C}$ & -1.68486500 & 1.24372700 & 2.09230700 \\
\hline $\mathrm{O}$ & -0.64430500 & 0.67917400 & 2.42274300 \\
\hline $\mathrm{O}$ & -2.88194300 & 0.85223400 & 2.46780600 \\
\hline $\mathrm{C}$ & -3.11776200 & -0.42015400 & 3.15608000 \\
\hline $\mathrm{C}$ & -2.77899200 & -1.56056900 & 2.20365900 \\
\hline
\end{tabular}




\begin{tabular}{|c|c|c|c|}
\hline $\mathrm{H}$ & -1.76731100 & -1.45509200 & 1.80068800 \\
\hline $\mathrm{H}$ & -2.85982000 & -2.51199900 & 2.74106200 \\
\hline $\mathrm{C}$ & -4.61566200 & -0.38344000 & 3.43061000 \\
\hline $\mathrm{H}$ & -5.16106300 & -0.25738200 & 2.49251000 \\
\hline $\mathrm{H}$ & -4.86107900 & 0.44059100 & 4.10697300 \\
\hline $\mathrm{H}$ & -4.92446400 & -1.32247500 & 3.89865000 \\
\hline $\mathrm{C}$ & -2.33504900 & -0.48145700 & 4.46560900 \\
\hline $\mathrm{H}$ & -2.73406600 & -1.30012300 & 5.07190300 \\
\hline $\mathrm{H}$ & -2.46537300 & 0.44884000 & 5.02674900 \\
\hline $\mathrm{H}$ & -1.26968300 & -0.64968600 & 4.30609900 \\
\hline $\mathrm{N}$ & -1.63518400 & 2.42541600 & 1.33913100 \\
\hline $\mathrm{H}$ & -3.70285600 & 2.37888500 & 1.14238200 \\
\hline $\mathrm{H}$ & -3.24698900 & 7.16520900 & -2.22626300 \\
\hline $\mathrm{H}$ & -0.99513400 & 6.23622700 & -1.77905200 \\
\hline $\mathrm{H}$ & -5.25471000 & 6.11064200 & -1.22383500 \\
\hline $\mathrm{H}$ & -4.99962500 & 4.10858900 & 0.22671200 \\
\hline $\mathrm{H}$ & -0.74506100 & 4.23811100 & -0.32071900 \\
\hline $\mathrm{C}$ & 2.84897600 & 5.42514800 & 1.55009900 \\
\hline $\mathrm{C}$ & 3.77569000 & 4.68485500 & 2.51802400 \\
\hline $\mathrm{C}$ & 3.12889000 & 3.39619400 & 3.03336900 \\
\hline $\mathrm{C}$ & 2.72145100 & 2.49096500 & 1.86879900 \\
\hline $\mathrm{C}$ & 1.76279200 & 3.24761900 & 0.91761600 \\
\hline $\mathrm{C}$ & 2.42813300 & 4.52205000 & 0.38716200 \\
\hline $\mathrm{H}$ & 2.23424400 & 3.63405500 & 3.62384700 \\
\hline $\mathrm{H}$ & 4.04212500 & 5.32943900 & 3.36070200 \\
\hline $\mathrm{H}$ & 4.71419600 & 4.43706500 & 2.00204800 \\
\hline $\mathrm{H}$ & 3.33833600 & 6.32556100 & 1.16710400 \\
\hline $\mathrm{H}$ & 1.95174000 & 5.75708700 & 2.08887000 \\
\hline
\end{tabular}




\begin{tabular}{|c|c|c|c|}
\hline $\mathrm{H}$ & 3.62186400 & 2.21699300 & 1.30862300 \\
\hline $\mathrm{H}$ & 1.72877400 & 5.03531200 & -0.28054300 \\
\hline $\mathrm{H}$ & 3.31138600 & 4.26521700 & -0.21385900 \\
\hline $\mathrm{H}$ & 3.80938300 & 2.84728600 & 3.69284300 \\
\hline $\mathrm{N}$ & 2.06875600 & 1.26571900 & 2.31554100 \\
\hline $\mathrm{H}$ & 1.05318900 & 1.34390500 & 2.43874700 \\
\hline $\mathrm{N}$ & 1.17869900 & 2.37953100 & -0.10161900 \\
\hline $\mathrm{H}$ & 0.15832800 & 2.27986600 & -0.07389400 \\
\hline $\mathrm{C}$ & 1.81455800 & 1.34188600 & -0.68200500 \\
\hline $\mathrm{C}$ & 3.16721300 & 1.38888500 & -1.09762100 \\
\hline $\mathrm{C}$ & 1.68089300 & -0.92916800 & -1.35747800 \\
\hline $\mathrm{H}$ & 0.06574900 & 0.20904400 & -0.64462500 \\
\hline $\mathrm{C}$ & 3.76765800 & 0.30456600 & -1.72126800 \\
\hline $\mathrm{H}$ & 3.66589200 & 2.34315200 & -1.03029000 \\
\hline $\mathrm{C}$ & 0.89854800 & -2.10098300 & -1.36807900 \\
\hline $\mathrm{C}$ & 3.03537200 & -0.95303800 & -1.75188100 \\
\hline $\mathrm{C}$ & 1.47996900 & -3.30322700 & -1.70053400 \\
\hline $\mathrm{H}$ & -0.14887600 & -2.02171600 & -1.08371400 \\
\hline $\mathrm{C}$ & 3.59999900 & -2.20815200 & -2.07022100 \\
\hline $\mathrm{C}$ & 2.84486700 & -3.36119000 & -2.03752200 \\
\hline $\mathrm{H}$ & 0.88415300 & -4.21120800 & -1.69180300 \\
\hline $\mathrm{H}$ & 4.65096500 & -2.27502800 & -2.32018400 \\
\hline $\mathrm{H}$ & 3.30428300 & -4.31509400 & -2.27530100 \\
\hline $\mathrm{N}$ & 1.10765300 & 0.22059100 & -0.88289300 \\
\hline $\mathrm{C}$ & 2.49473700 & 0.01599500 & 2.06021000 \\
\hline $\mathrm{C}$ & 3.82473900 & -0.32313800 & 1.76989300 \\
\hline $\mathrm{C}$ & 1.87340500 & -2.29282200 & 1.87870300 \\
\hline $\mathrm{C}$ & 4.19739600 & -1.62583200 & 1.43089900 \\
\hline
\end{tabular}




\begin{tabular}{|c|c|c|c|}
\hline $\mathrm{H}$ & 4.54803800 & 0.47537200 & 1.76953400 \\
\hline $\mathrm{C}$ & 3.19509700 & -2.68488800 & 1.57326000 \\
\hline $\mathrm{N}$ & 1.56462500 & -0.96502800 & 2.07671600 \\
\hline $\mathrm{C}$ & 0.84080100 & -3.23738600 & 1.99651800 \\
\hline $\mathrm{C}$ & 1.11676100 & -4.57884900 & 1.85396900 \\
\hline $\mathrm{C}$ & 2.43435400 & -5.00108500 & 1.62727700 \\
\hline $\mathrm{H}$ & 2.66517600 & -6.05885900 & 1.56800200 \\
\hline $\mathrm{C}$ & 3.44699500 & -4.07334900 & 1.49872600 \\
\hline $\mathrm{H}$ & 4.45484100 & -4.43824100 & 1.37521600 \\
\hline $\mathrm{C}$ & -6.34665000 & -4.18756400 & -0.08910900 \\
\hline $\mathrm{C}$ & -7.04063100 & -3.04932500 & -0.47496300 \\
\hline $\mathrm{C}$ & -6.37421700 & -1.93139700 & -0.98805300 \\
\hline $\mathrm{C}$ & -4.98659400 & -2.01617300 & -1.08180600 \\
\hline $\mathrm{C}$ & -4.25075400 & -3.15171100 & -0.72091500 \\
\hline $\mathrm{C}$ & -4.96070700 & -4.24067300 & -0.21866500 \\
\hline $\mathrm{H}$ & -6.88584400 & -5.04234700 & 0.30673000 \\
\hline $\mathrm{H}$ & -8.12288100 & -3.02205400 & -0.38062600 \\
\hline $\mathrm{C}$ & -7.15563500 & -0.71103100 & -1.43898300 \\
\hline $\mathrm{C}$ & -2.74822600 & -3.16835500 & -0.94109900 \\
\hline $\mathrm{H}$ & -4.43072800 & -5.14090700 & 0.07583500 \\
\hline $\mathrm{C}$ & -8.13699300 & -1.07708600 & -2.55946900 \\
\hline $\mathrm{H}$ & -6.44895500 & 0.01790200 & -1.84902100 \\
\hline $\mathrm{C}$ & -7.88659300 & -0.05638600 & -0.26186000 \\
\hline $\mathrm{H}$ & -8.41788000 & 0.84075400 & -0.59580300 \\
\hline $\mathrm{H}$ & -7.18192700 & 0.22458900 & 0.52448200 \\
\hline $\mathrm{H}$ & -8.62713400 & -0.74194800 & 0.16434800 \\
\hline $\mathrm{H}$ & -8.65636300 & -0.18190500 & -2.91560300 \\
\hline $\mathrm{H}$ & -8.89567900 & -1.78222300 & -2.20412300 \\
\hline
\end{tabular}




\begin{tabular}{|c|c|c|c|}
\hline $\mathrm{H}$ & -7.61935900 & -1.53676800 & -3.40608500 \\
\hline $\mathrm{H}$ & -2.36781400 & -2.17331900 & -0.67382000 \\
\hline $\mathrm{C}$ & -2.44881900 & -3.39938600 & -2.43007300 \\
\hline $\mathrm{C}$ & -2.02541400 & -4.20844900 & -0.08519200 \\
\hline $\mathrm{H}$ & -1.36873300 & -3.39969800 & -2.61828100 \\
\hline $\mathrm{H}$ & -2.89978200 & -2.61610000 & -3.04423200 \\
\hline $\mathrm{H}$ & -2.85045600 & -4.36641800 & -2.75191200 \\
\hline $\mathrm{H}$ & -2.26122000 & -5.22901100 & -0.40737100 \\
\hline $\mathrm{H}$ & -2.28978700 & -4.11726900 & 0.97467700 \\
\hline $\mathrm{H}$ & -0.94070700 & -4.07967200 & -0.17632400 \\
\hline $\mathrm{H}$ & 0.89327900 & 3.55321900 & 1.51857100 \\
\hline $\mathrm{H}$ & 0.58763400 & -0.69271300 & 2.21833600 \\
\hline $\mathrm{H}$ & -3.48062600 & -1.57158700 & 1.36570300 \\
\hline $\mathrm{H}$ & -0.16835500 & -2.89423700 & 2.20732900 \\
\hline $\mathrm{H}$ & 0.31470400 & -5.30480100 & 1.94233500 \\
\hline $\mathrm{C}$ & 6.43260800 & -0.74536700 & 0.96283700 \\
\hline $\mathrm{C}$ & 5.98335800 & -3.02677900 & 0.29556600 \\
\hline $\mathrm{C}$ & 7.70353100 & -1.38444400 & 0.41318400 \\
\hline $\mathrm{H}$ & 6.05633200 & 0.03093100 & 0.27981400 \\
\hline $\mathrm{H}$ & 6.56646800 & -0.30514200 & 1.95569500 \\
\hline $\mathrm{C}$ & 7.14386800 & -2.45782800 & -0.51749300 \\
\hline $\mathrm{H}$ & 6.35217800 & -3.78225900 & 1.00018300 \\
\hline $\mathrm{H}$ & 5.20154300 & -3.46755500 & -0.32571400 \\
\hline $\mathrm{H}$ & 8.27929100 & -1.84391600 & 1.22273200 \\
\hline $\mathrm{H}$ & 8.34291300 & -0.65742100 & -0.09126100 \\
\hline $\mathrm{H}$ & 7.86796100 & -3.22419600 & -0.79939900 \\
\hline $\mathrm{H}$ & 6.75846100 & -1.98406400 & -1.42771200 \\
\hline $\mathrm{C}$ & 5.43633700 & -0.29614500 & -3.49945600 \\
\hline
\end{tabular}




$\begin{array}{llll}\mathrm{C} & 5.74545500 & 1.68564800 & -2.15146100 \\ \mathrm{C} & 6.31595500 & 0.71973600 & -4.22965800 \\ \mathrm{H} & 6.02978300 & -1.18534500 & -3.26102100 \\ \mathrm{H} & 4.56085700 & -0.61030100 & -4.07295700 \\ \mathrm{C} & 6.94307800 & 1.50088700 & -3.07453600 \\ \mathrm{H} & 5.12000800 & 2.51481100 & -2.51322000 \\ \mathrm{H} & 6.01006900 & 1.88737800 & -1.10740300 \\ \mathrm{H} & 5.69920900 & 1.38661000 & -4.84054700 \\ \mathrm{H} & 7.04580600 & 0.23475900 & -4.88045600 \\ \mathrm{H} & 7.38358100 & 2.45237100 & -3.37669500 \\ \mathrm{H} & 7.71631200 & 0.90088700 & -2.58134800 \\ \mathrm{~N} & 5.02076000 & 0.41028800 & -2.26070800 \\ \mathrm{~N} & 5.46456900 & -1.85167300 & 1.01718700\end{array}$




\section{Parameters for OPLS3 Conformer Searches}

The following parameters where used for the conformational searches:

Potential (parameters)

Force field $=$ OPLS3

Solvent $=$ none

Electrostatic treatment $=$ constant dielectric

Dielectric constant $=1$

Charges from force field

Cutoff $=$ normal

Van der Waals $=7.0$

Electrostatic $=12.0$

H-bond $=4$

$\underline{\text { Mini (parameters) }}$

Method $=$ PRCG

Maximum interactions $=2500$

Converge on $=$ Gradient

Convergence threshold $=0.05$

\section{CSearch (parameters)}

Method $=$ Mixed torsional/Low-mode sampling

Torsion sampling option $=$ intermediate

Maximum number of steps $=1000$ (using 100 steps per rotatable bond)

Number of structures saved from search $=100$

Energy window for saving structures $=5.02 \mathrm{kcal} / \mathrm{mol}$

Eliminate redundant conformers using: Maximum atom deviation Cutoff $=0.5$ Angstrom

Probability of a torsion rotation $/$ molecule transition $=0.5$

Minimum distance for low-mode move $=3.0$

Maximum distance for low-mode move $=6.0$
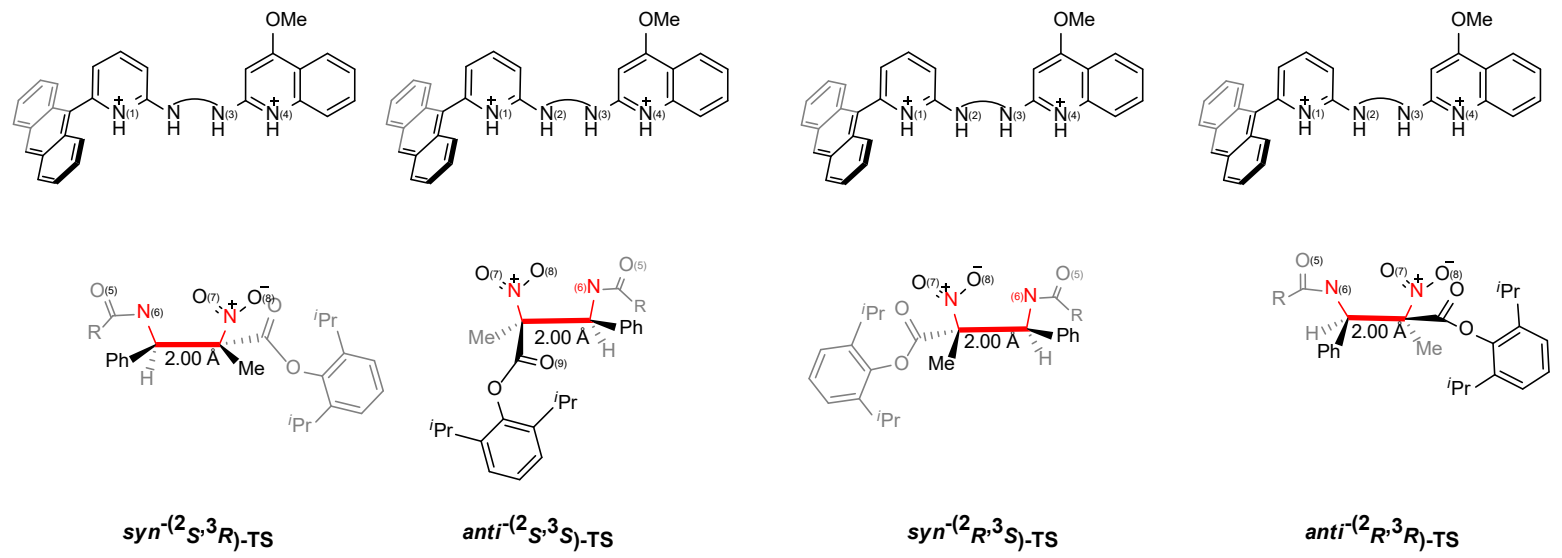

$\mathrm{R}=\mathrm{O}^{t} \mathrm{Bu}$

$\left.\operatorname{anti}^{-(2} S^{, 3} S\right)-\mathrm{TS}$

$\left.\operatorname{syn}^{-(2} R^{, 3} S\right)-\mathrm{TS}$

anti $\left.^{-(2} R^{, 3} R\right)$-TS

Figure S2. Strategy for OPLS3 Conformer Searches. 


\section{Quantum Theory of Atoms in Molecules (QTAIM)}

It is evident from the literature that there are other important relationships between energetic topological parameters and the $\nabla^{2} \rho\left(\mathbf{r}_{\mathbf{c}}\right)$ at CPs. One of the important relationships is the local form of virial theorem: ${ }^{14}$

$1 / 4 \nabla^{2} \rho(\mathrm{rc})=2 \mathrm{G}(\mathrm{rc})+\mathrm{V}(\mathrm{rc})$

$\mathrm{H}(\mathrm{rc})=\mathrm{V}(\mathrm{rc})+\mathrm{G}(\mathrm{rc})$

where $G\left(\mathbf{r}_{\mathbf{c}}\right), V\left(\mathbf{r}_{\mathbf{c}}\right)$, and $H\left(\mathbf{r}_{\mathbf{c}}\right)$ are the kinetic energy, potential energy, and the total electron energy densities respectively. $G\left(\mathbf{r}_{\mathbf{c}}\right)$ is a positive quantity and $V\left(\mathbf{r}_{\mathbf{c}}\right)$ is a negative quantity. The balance between the kinetic electron energy density $G\left(\mathbf{r}_{\mathbf{c}}\right)$ and the potential electron energy density $V\left(\mathbf{r}_{\mathbf{c}}\right)$ reveals the nature of the interaction. If $\left|V\left(\mathbf{r}_{\mathbf{c}}\right)\right|>2 \mathrm{G}\left(\mathbf{r}_{\mathbf{c}}\right)$, then the interaction is covalent in nature. If $\left|V\left(\mathbf{r}_{\mathbf{c}}\right)\right|$ is one time more than the $G\left(\mathbf{r}_{\mathbf{c}}\right)$ then $\nabla^{2} \rho\left(\mathbf{r}_{\mathbf{c}}\right)$ is positive and $H\left(\mathbf{r}_{\mathbf{c}}\right)$ is negative. In this situation, both $\nabla^{2} \rho\left(\mathbf{r}_{\mathbf{c}}\right)$ and $H\left(\mathbf{r}_{\mathbf{c}}\right)$ have been used to characterize bonding interaction. Similarly, the ratio of $-\mathrm{G}(\mathrm{rc}) \mathrm{V}(\mathrm{rc})-\mathrm{G}(\mathrm{rc}) \mathrm{V}(\mathrm{rc})$ has also been employed to classify the bonding interaction. ${ }^{15}$ If this ratio is greater than 1 then the nature of the interaction is purely non-covalent. Different criteria for describing the nature of bonding using the topological parameters such as $\rho\left(\mathbf{r}_{\mathbf{c}}\right), \nabla^{2} \rho\left(\mathbf{r}_{\mathbf{c}}\right), V\left(\mathbf{r}_{\mathbf{c}}\right), G\left(\mathbf{r}_{\mathbf{c}}\right)$, and $H\left(\mathbf{r}_{\mathbf{c}}\right)$ have been summarized in previous reports. ${ }^{16,17}$ 
The images below showcase different angles of a QTAIM analysis performed on transition state syn-(2S,3R)-TS.
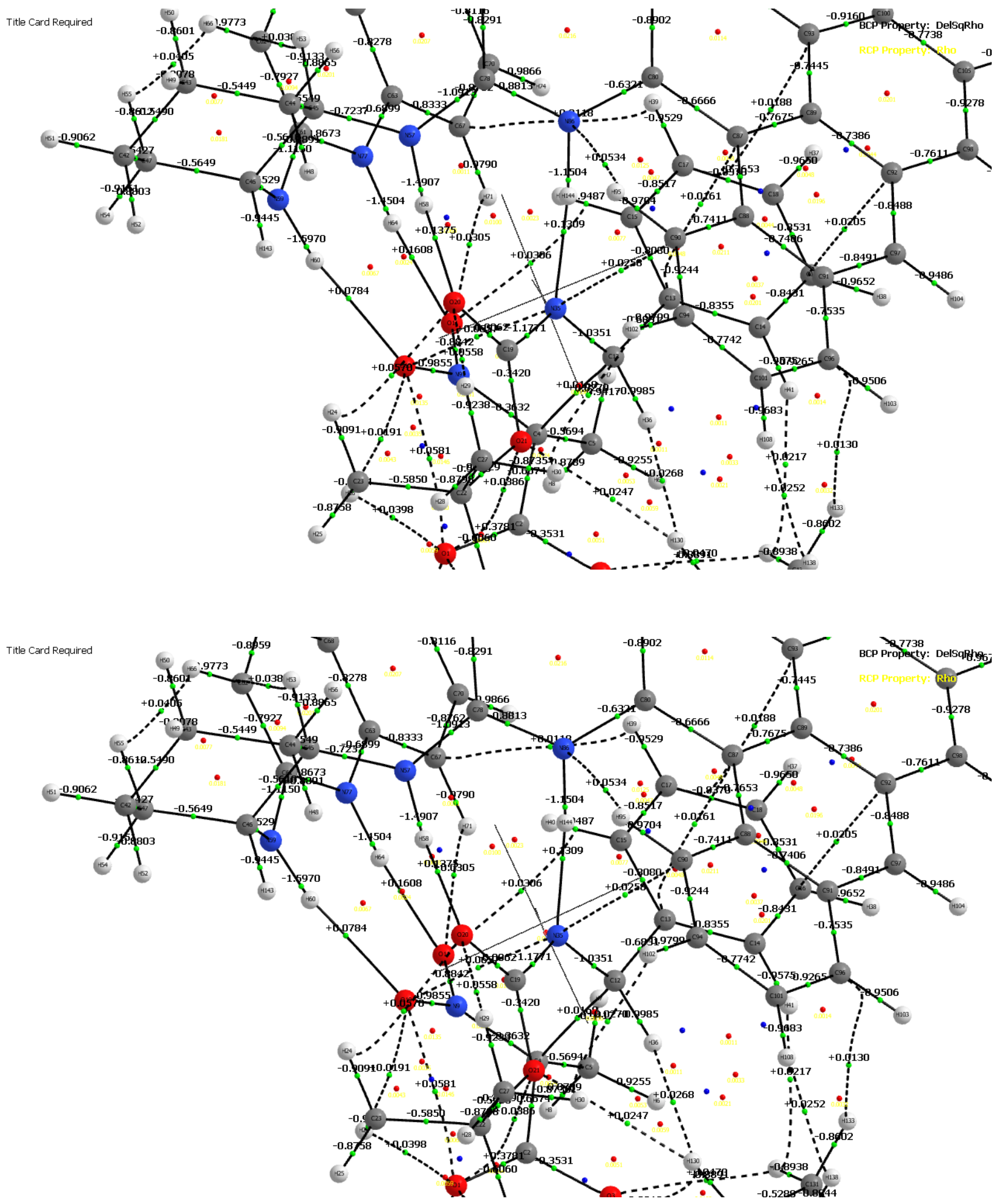


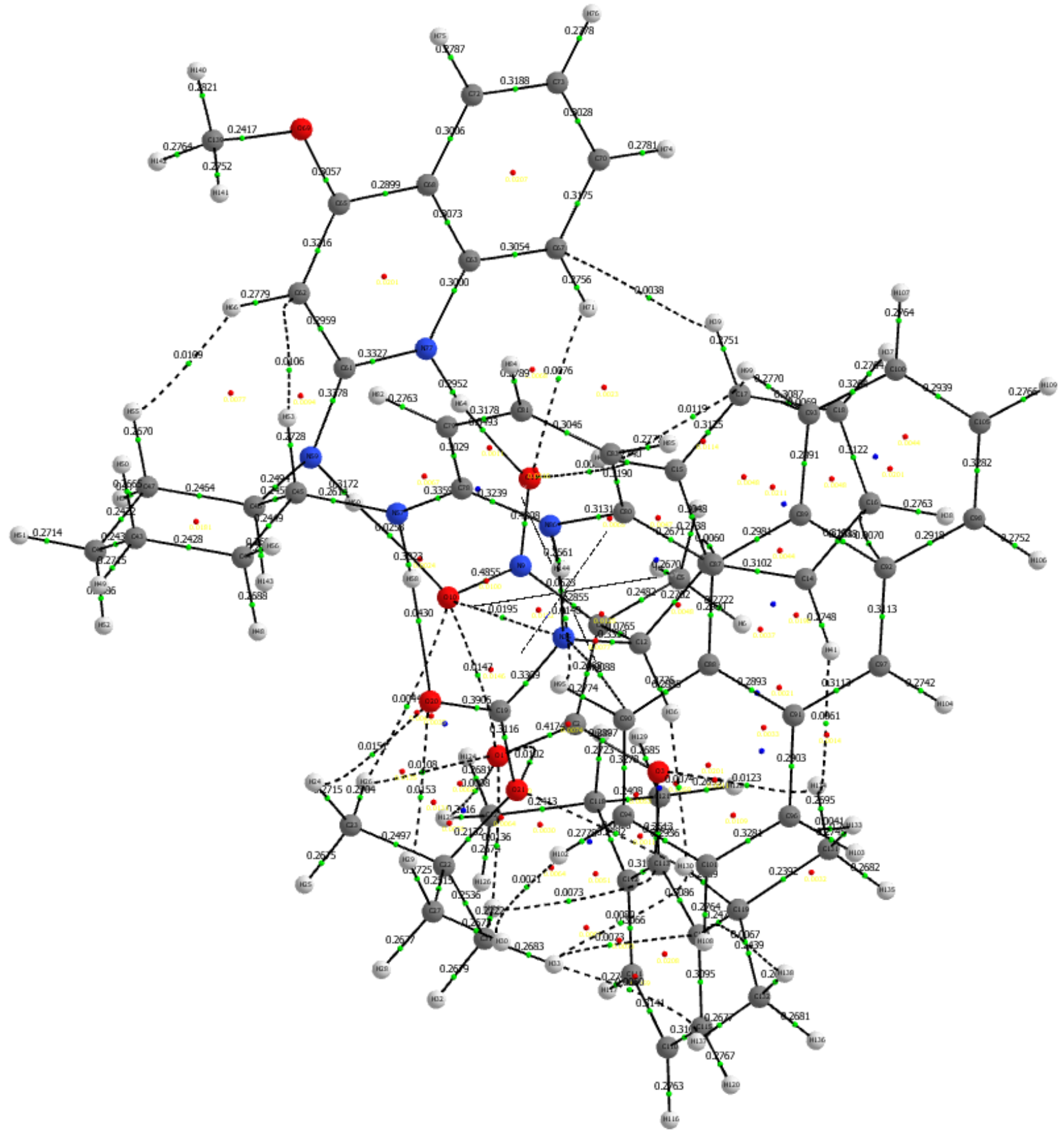



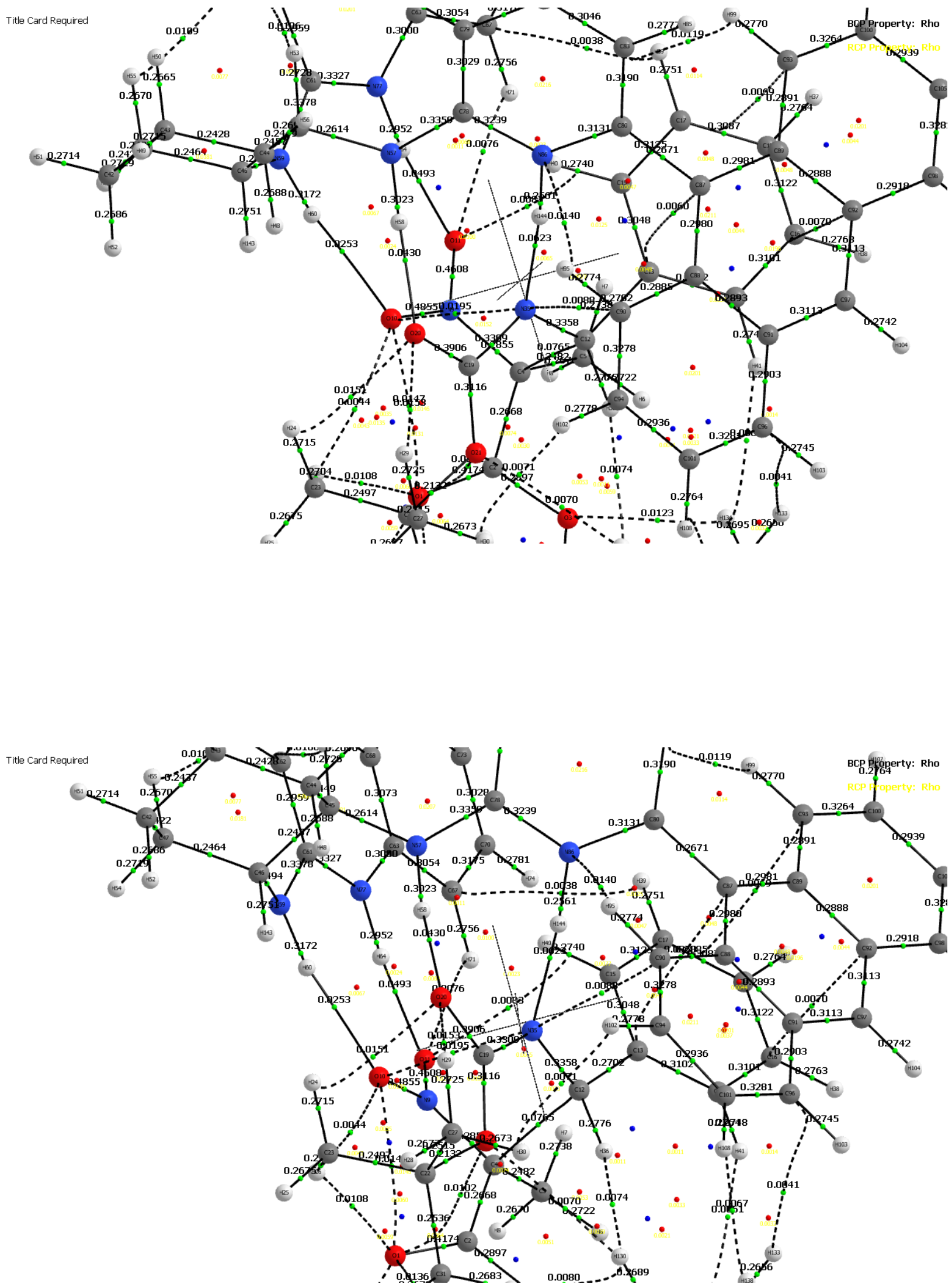


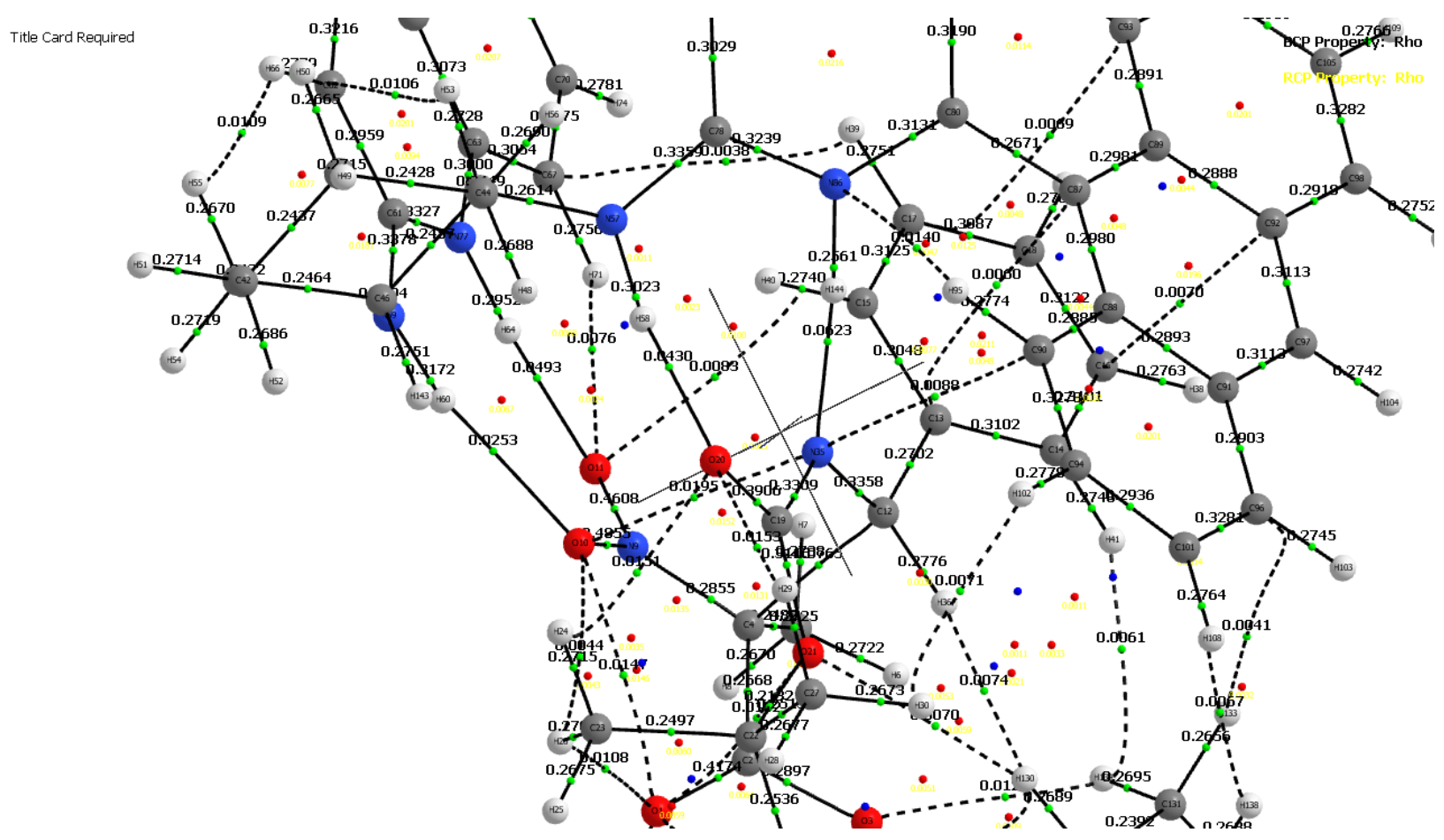


NCI Plots: Additional, Enlarged Views

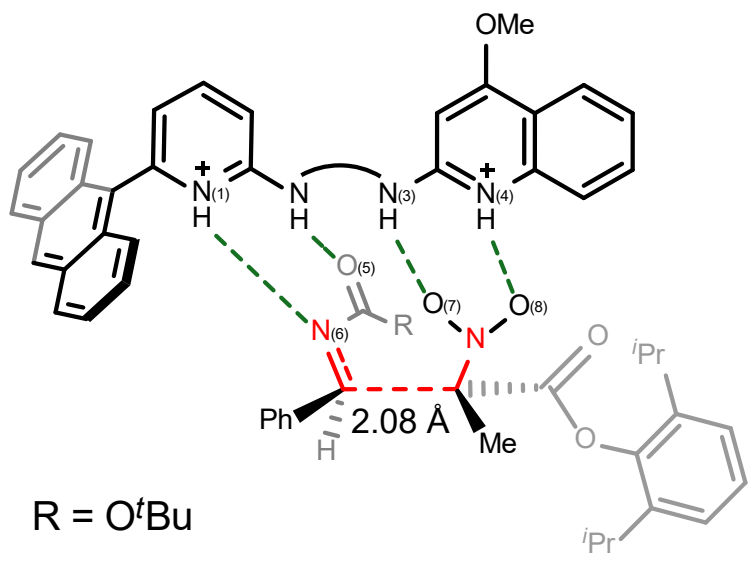

$\boldsymbol{s y n}^{-}(\mathbf{2 S}, 3 \boldsymbol{B})^{-\mathrm{TS}} \Delta \mathrm{G}=0.0 \mathrm{kcal} \mathrm{mol}^{-1}$

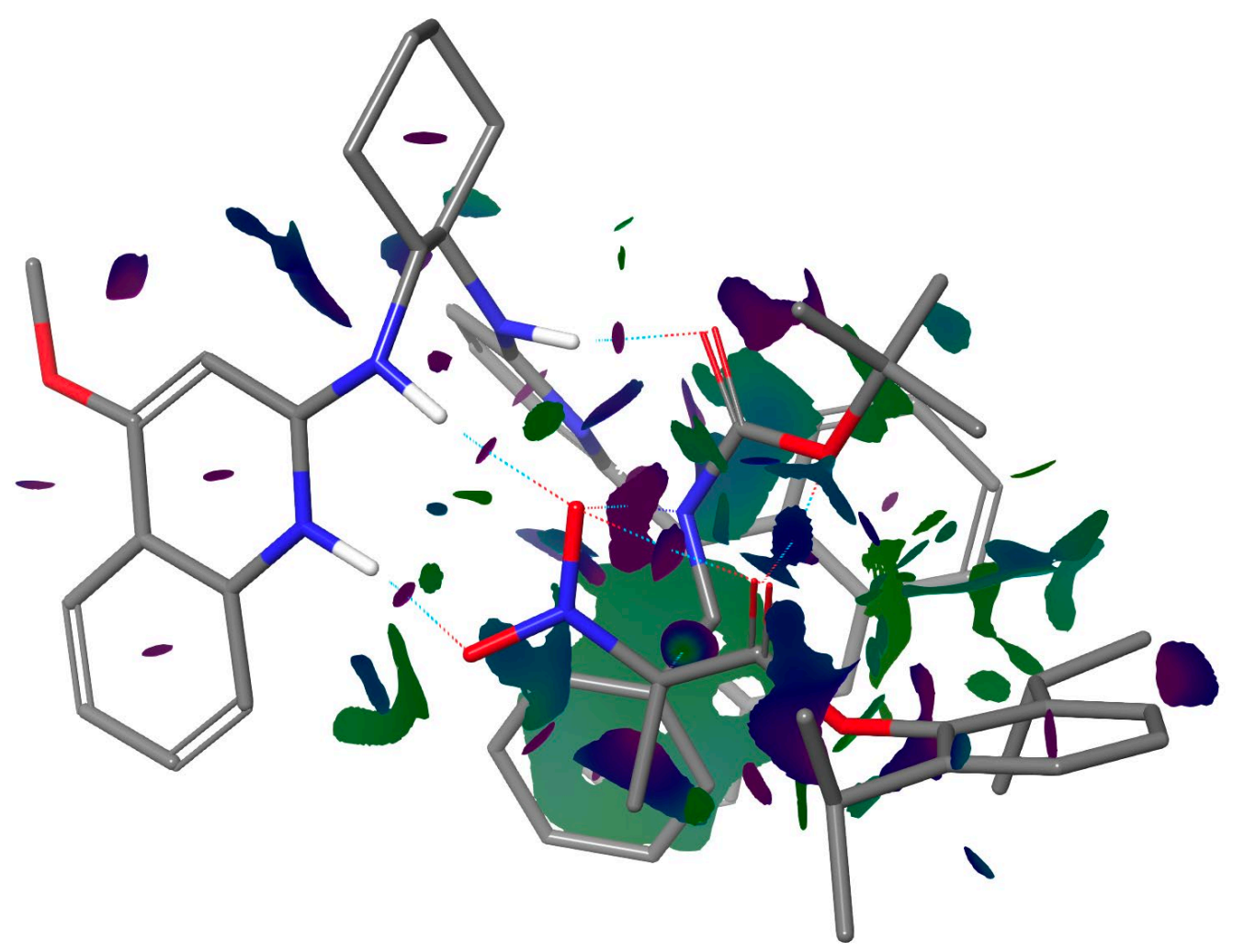




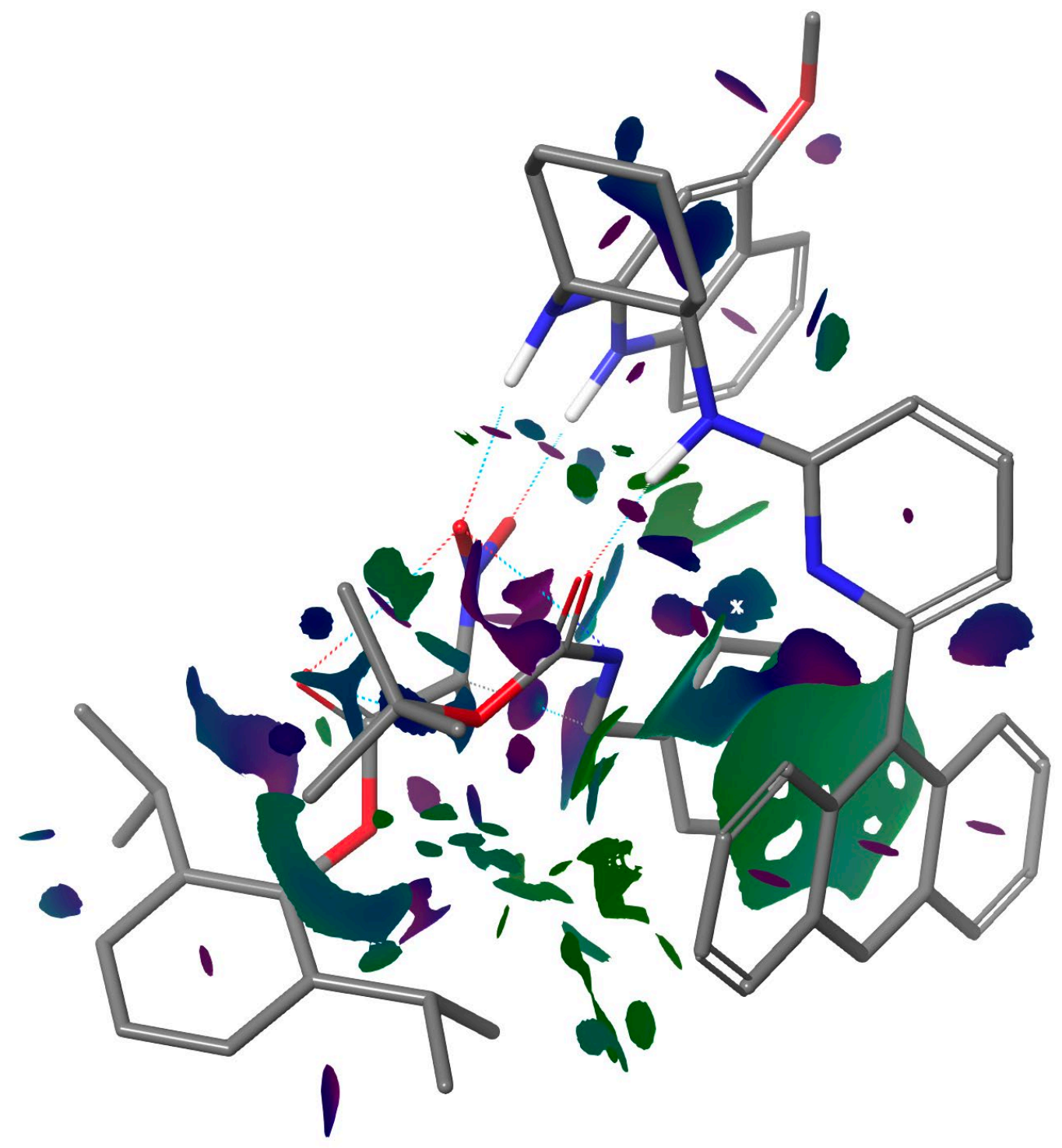




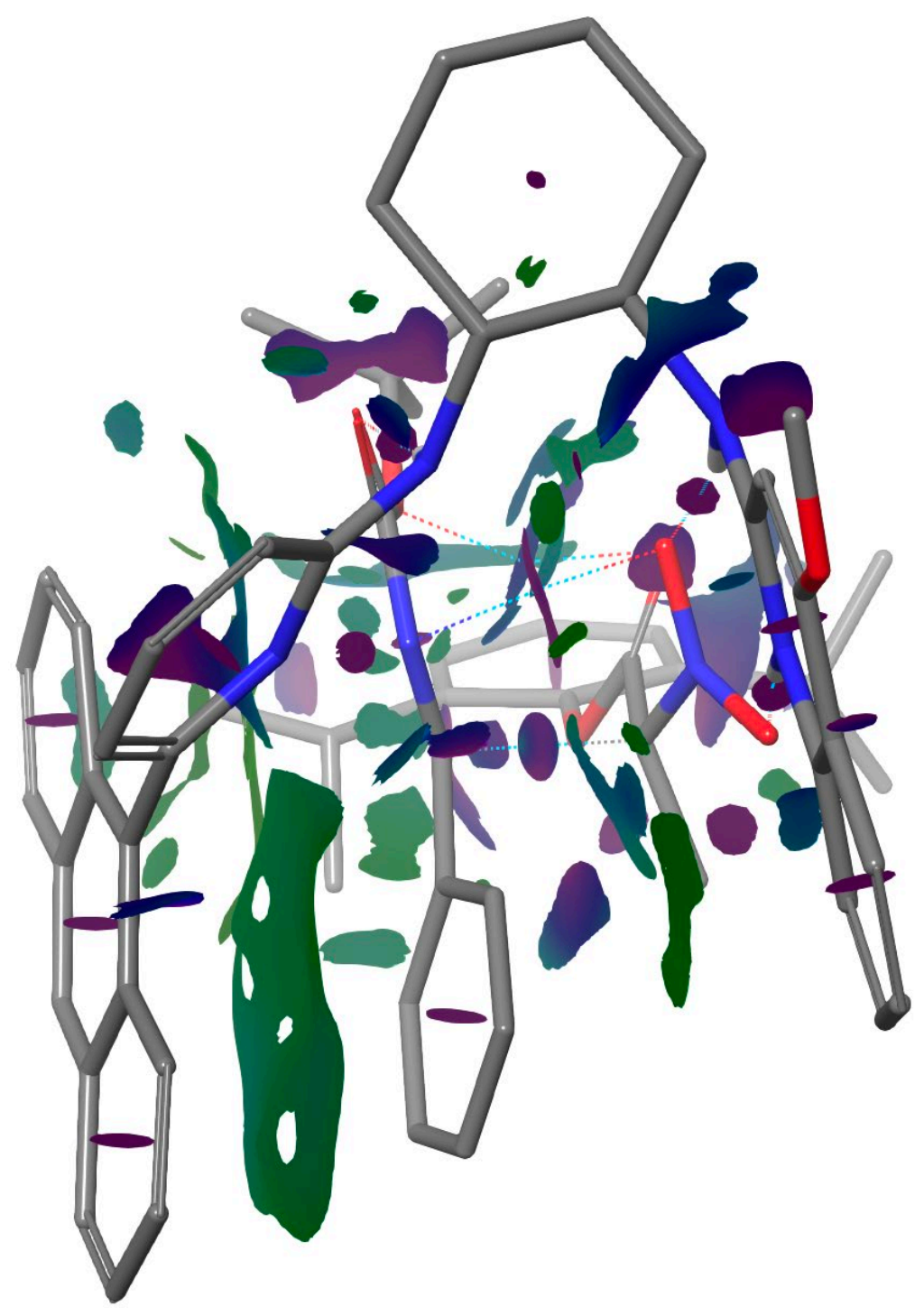




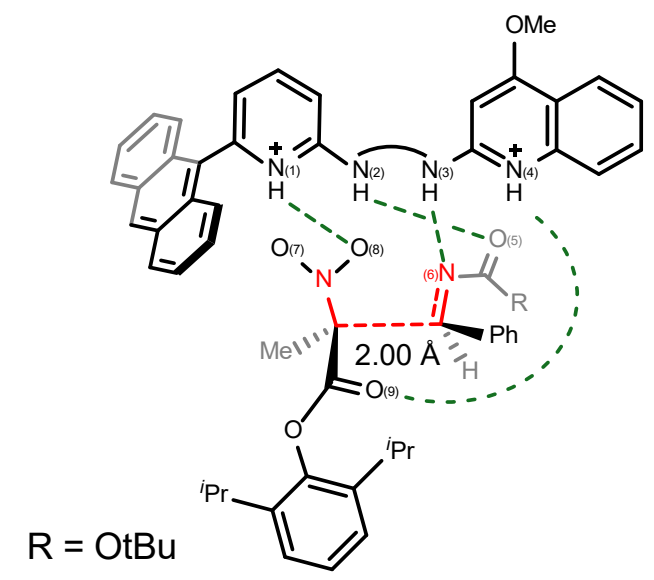

anti- $^{-}(2 S, 3 S)^{-T S} \Delta \mathrm{G}=0.5 \mathrm{kcal} \mathrm{mol}{ }^{-1}$

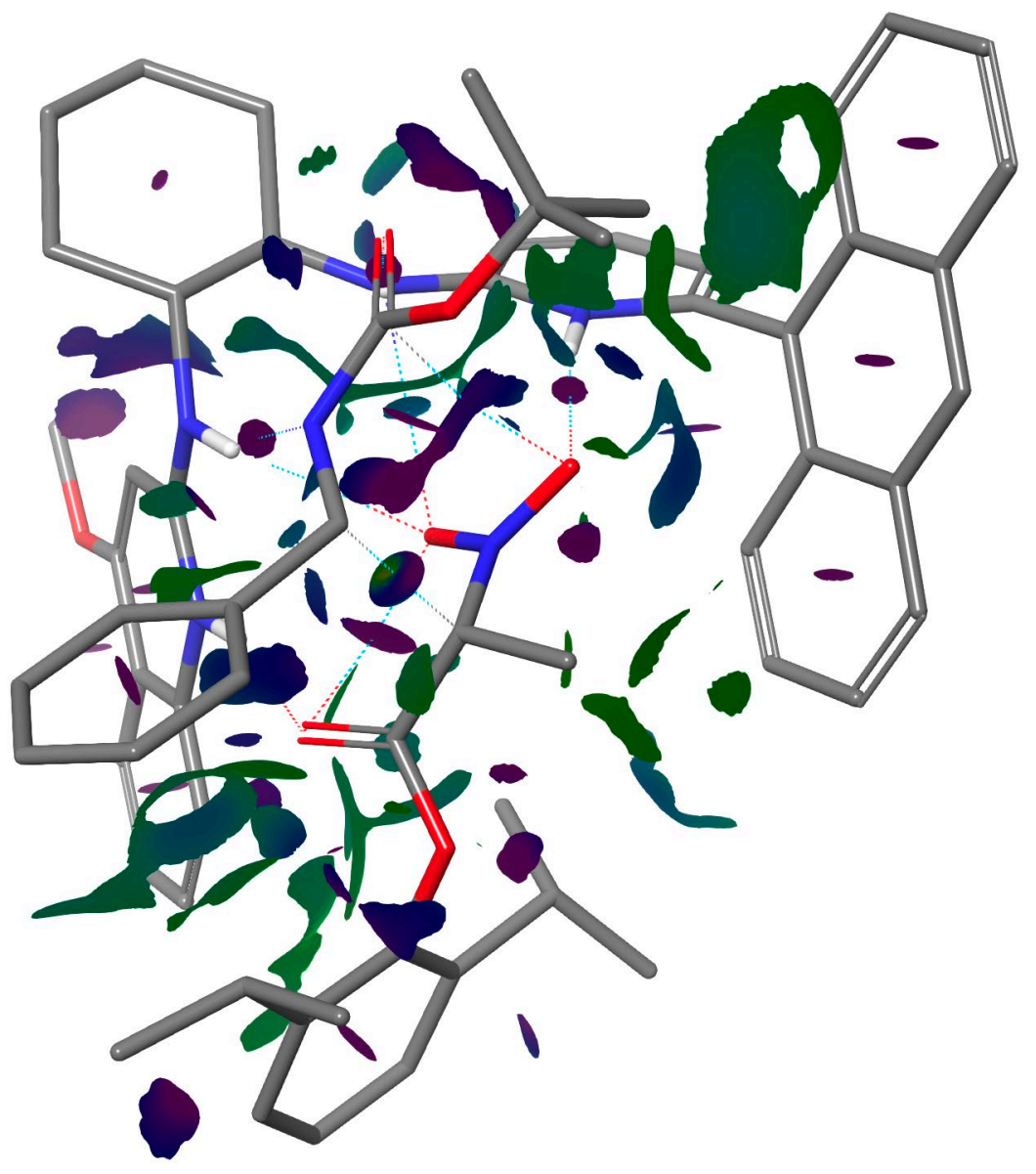




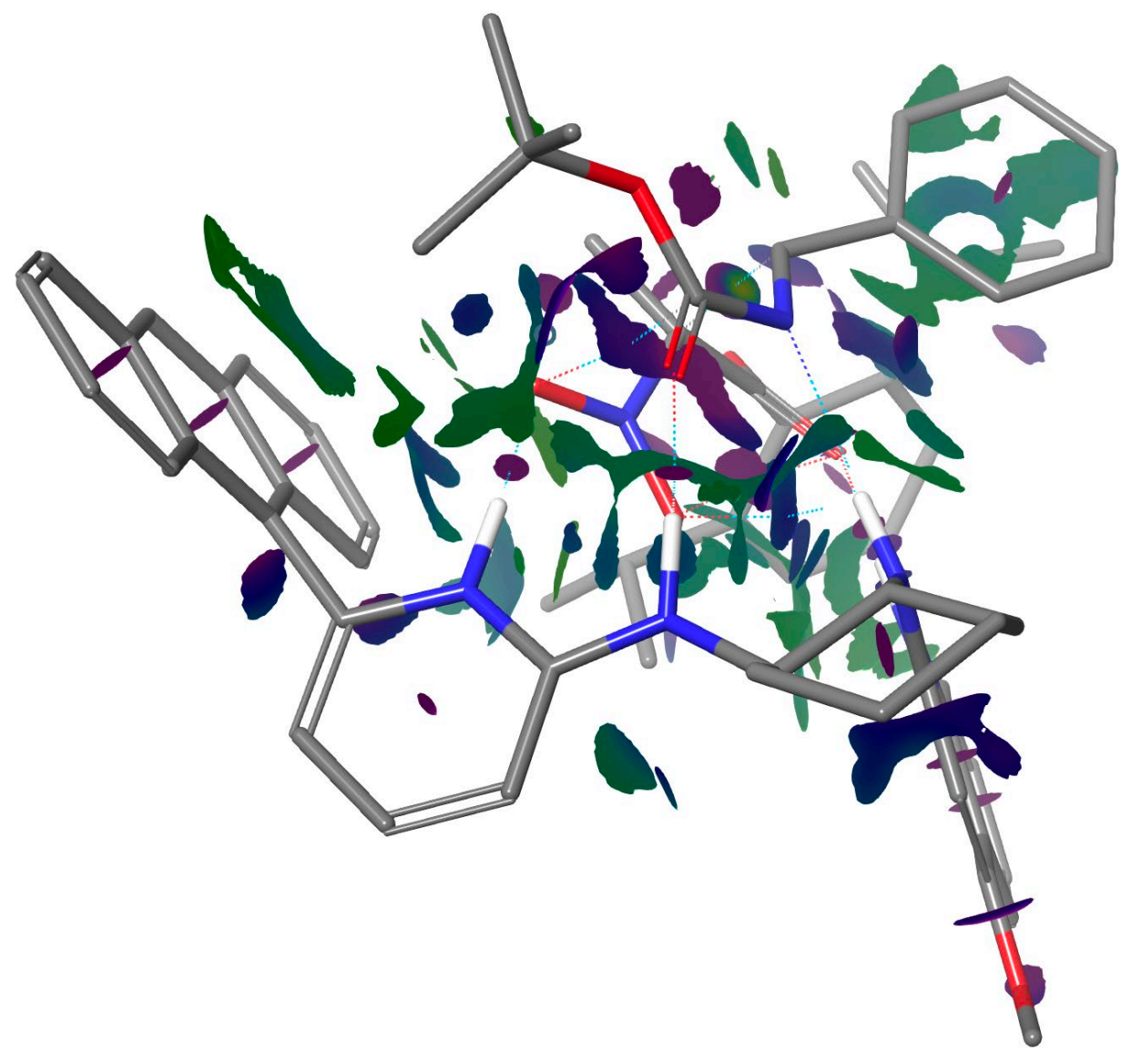




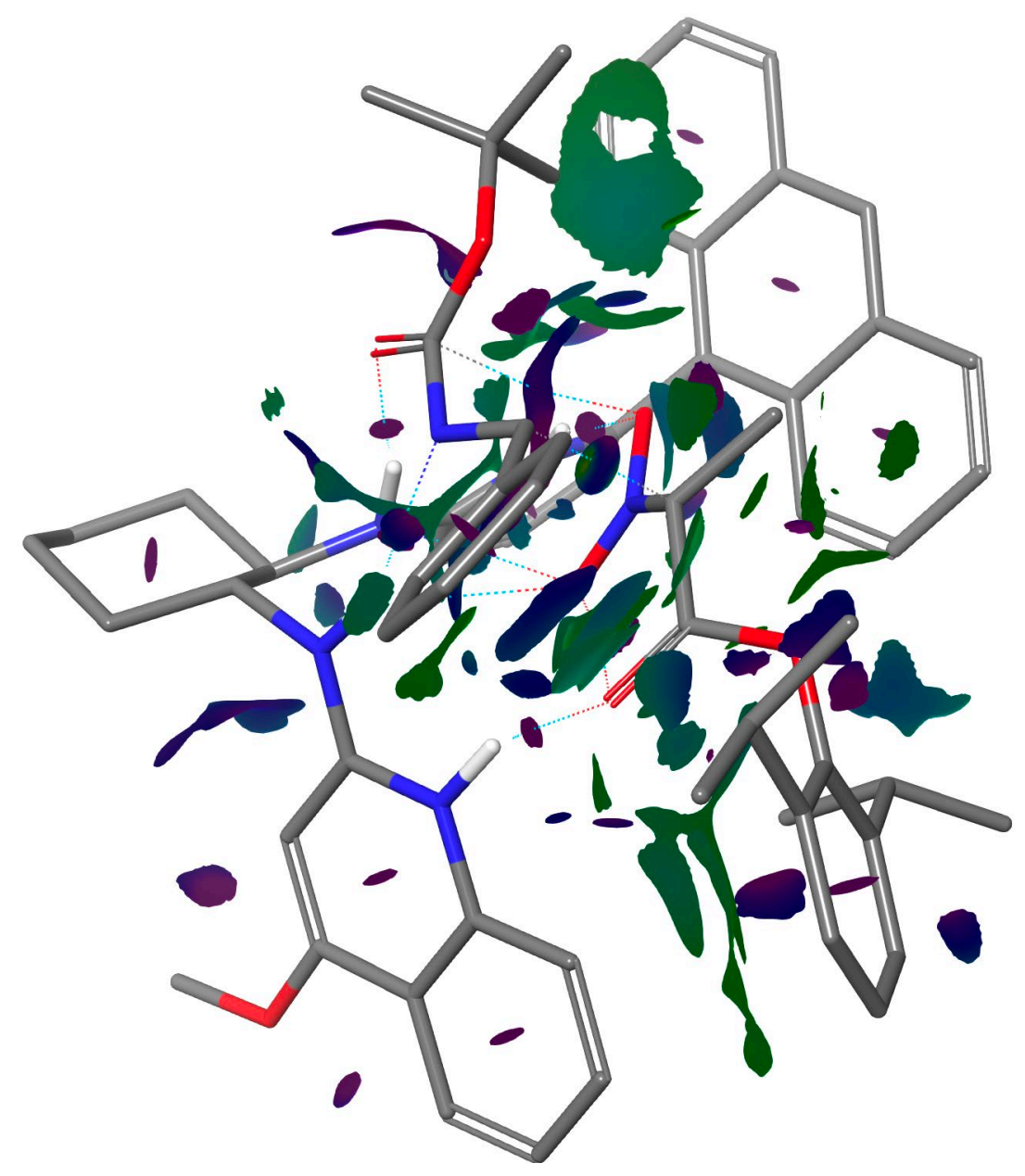




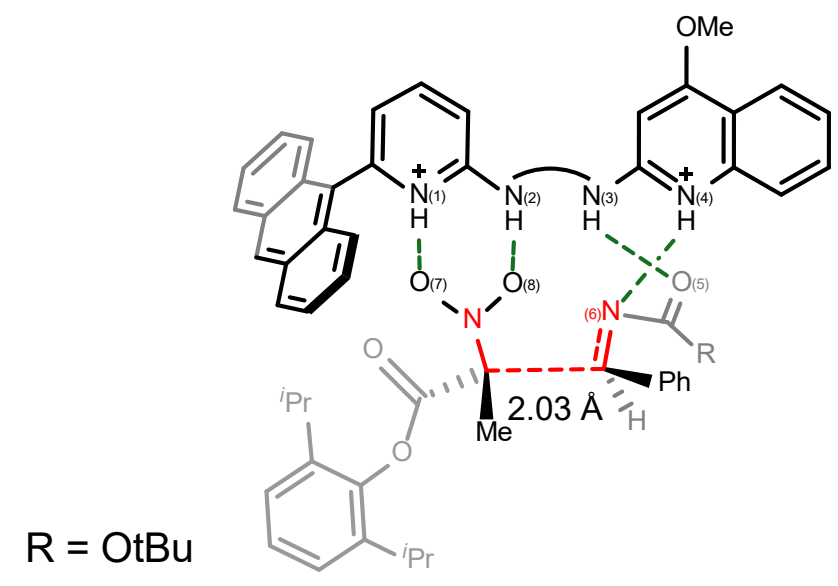

$\operatorname{syn}^{-}(\mathbf{2} \boldsymbol{R}, 3 S)-\mathbf{T S} \Delta \mathrm{G}=1 \cdot 1 \mathrm{kcal} \mathrm{mol}^{-1}$

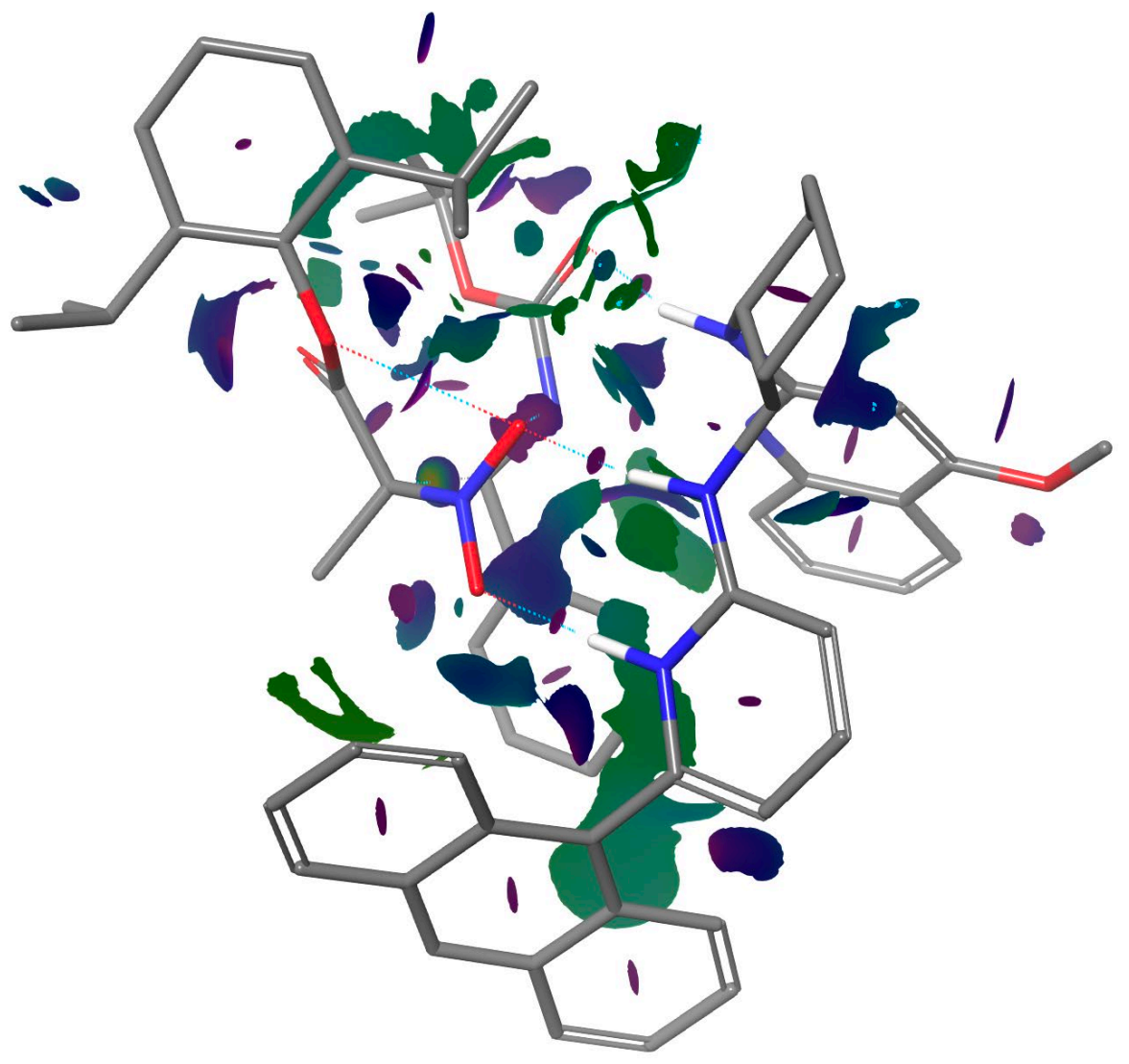




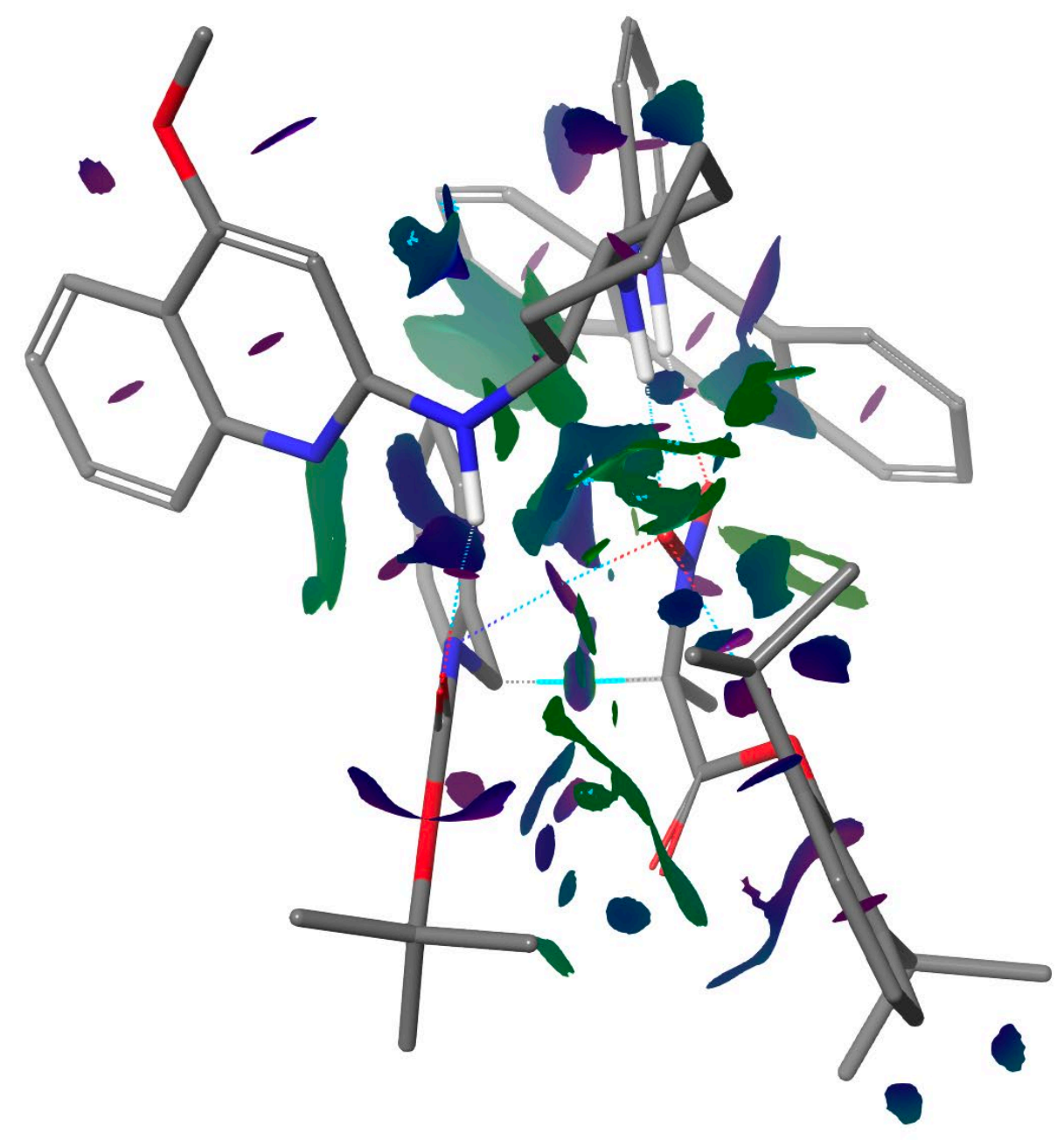




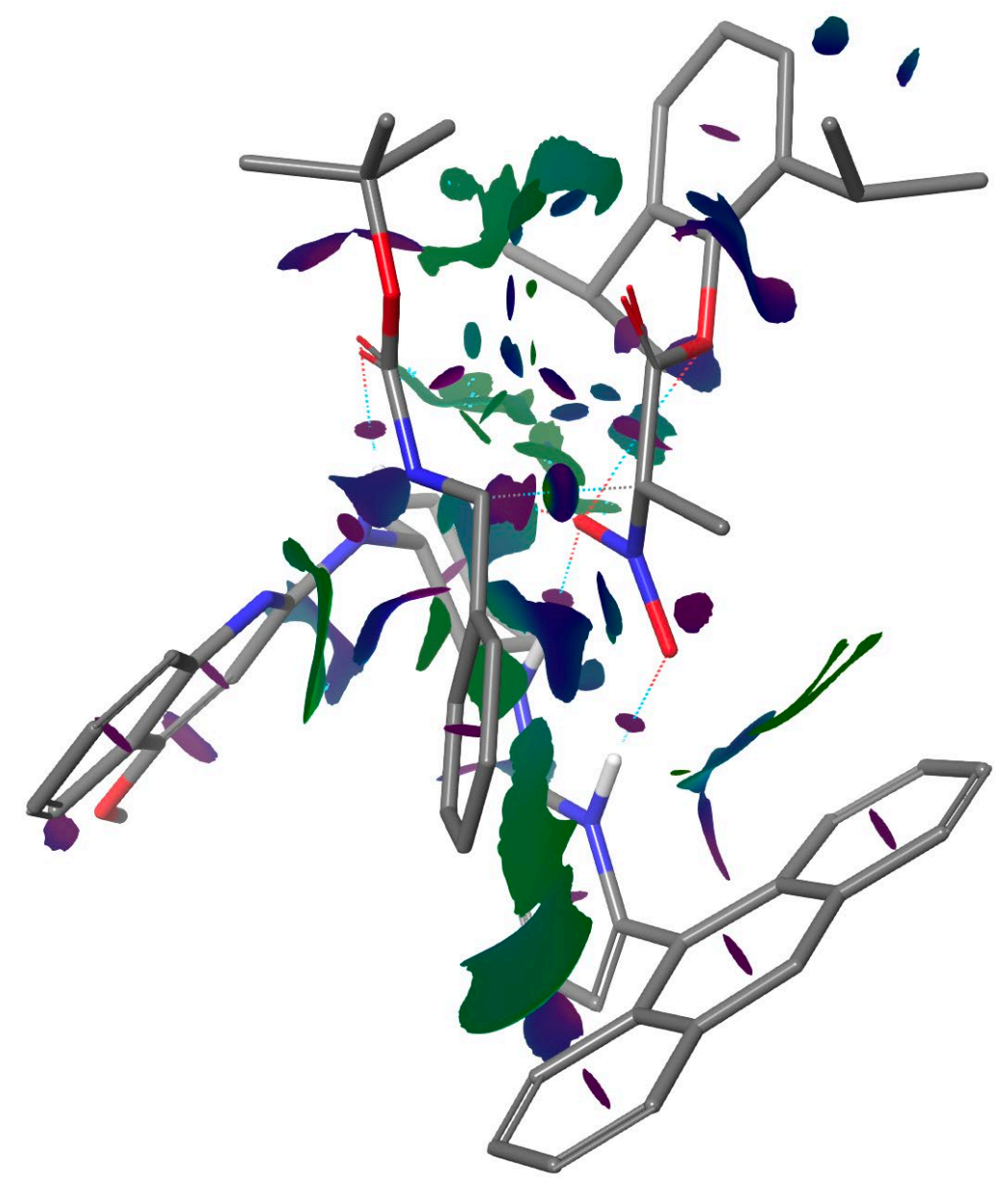




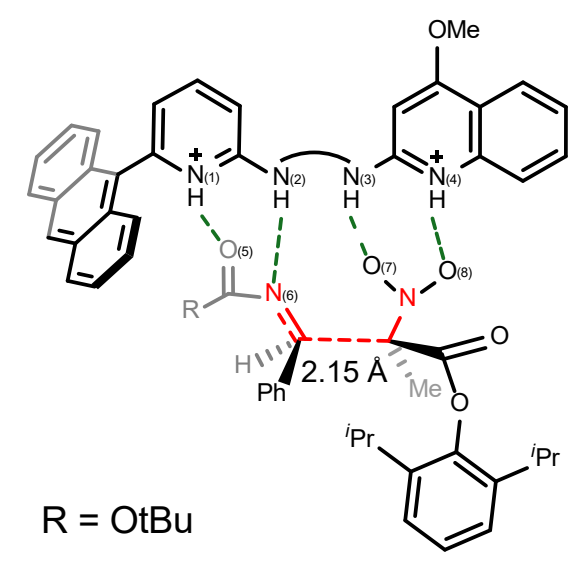

anti- $(2 R, 3 R)^{-T S} \Delta \mathrm{G}=2 \cdot 0 \mathrm{kcal} \mathrm{mol}^{-1}$

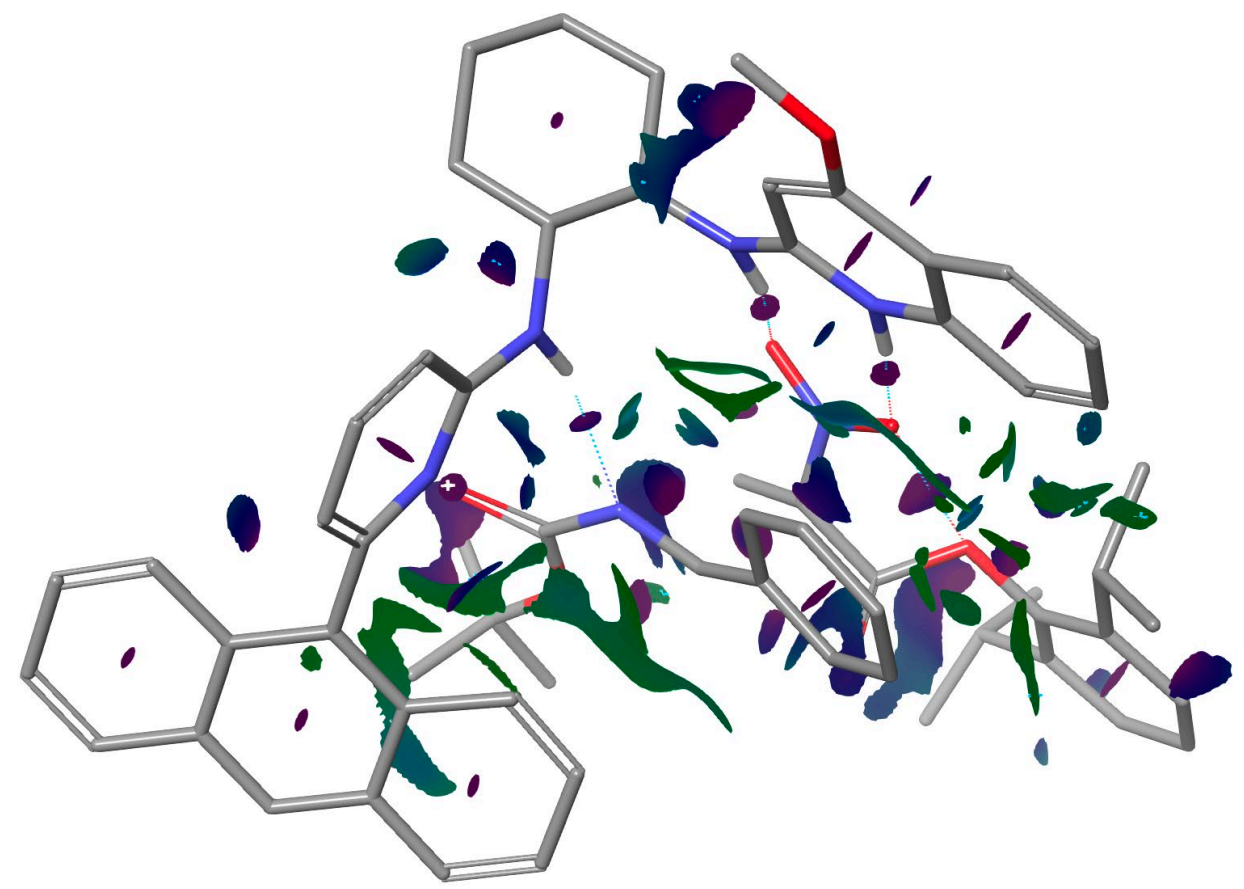



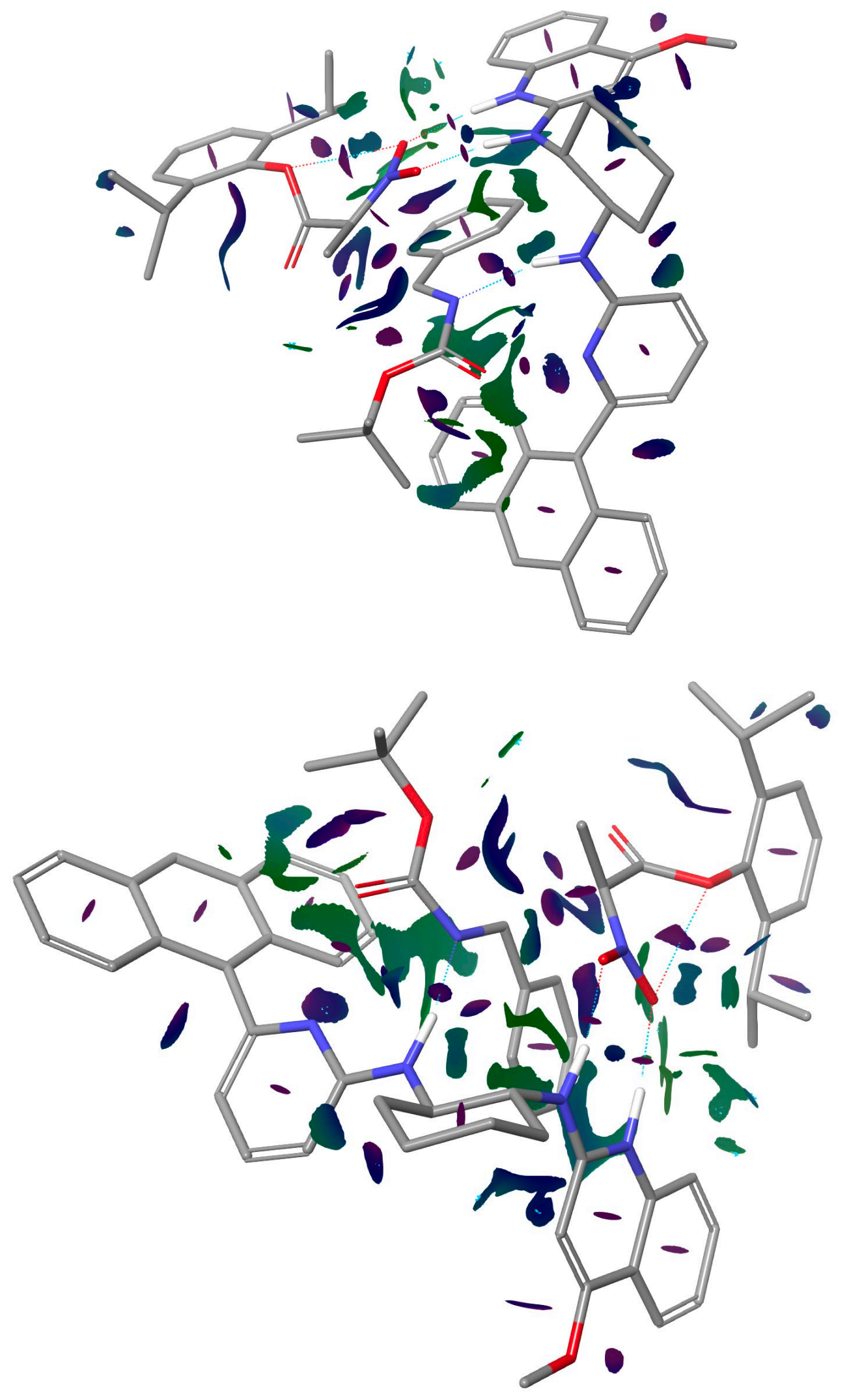


\section{References}

1 M. J. Frisch, G. W. T., H. B. Schlegel, G. E. Scuseria, M. A. Robb, J. R. Cheeseman, G. Scalmani, V. Barone, G. A. Petersson, H. Nakatsuji, X. Li, M. Caricato, A. Marenich, J. Bloino, B. G. Janesko, R. Gomperts, B. Mennucci, H. P. Hratchian, J. V. Ortiz, A. F. Izmaylov, J. L. Sonnenberg, D. Williams-Young, F. Ding, F. Lipparini, F. Egidi, J. Goings, B. Peng, A. Petrone, T. Henderson, D. Ranasinghe, V. G. Zakrzewski, J. Gao, N. Rega, G. Zheng, W. Liang, M. Hada, M. Ehara, K. Toyota, R. Fukuda, J. Hasegawa, M. Ishida, T. Nakajima, Y. Honda, O. Kitao, H. Nakai, T. Vreven, K. Throssell, J. A. Montgomery, Jr., J. E. Peralta, F. Ogliaro, M. Bearpark, J. J. Heyd, E. Brothers, K. N. Kudin, V. N. Staroverov, T. Keith, R. Kobayashi, J. Normand, K. Raghavachari, A. Rendell, J. C. Burant, S. S. Iyengar, J. Tomasi, M. Cossi, J. M. Millam, M. Klene, C. Adamo, R. Cammi, J. W. Ochterski, R. L. Martin, K. Morokuma, O. Farkas, J. B. Foresman, and D. J. Fox, Gaussian, Inc., Wallingford CT, 2016.

2 Zhao, Y. \& Truhlar, D. G. The M06 Suite of Density Functionals for Main Group Thermochemistry, Thermochemical Kinetics, Non-covalent Interactions, Excited States, and Transition Elements. Theor. Chem. Acc. 120, 215-241, (2008). Walker, M., Harvey, A. J. A., Sen, A. \& Dessent, C. E. H. Performance of M06, M06-2X, and M06-HF Density Functionals for Conformationally Flexible Anionic Clusters: M06 Functionals Perform Better than B3LYP for a Model System with Dispersion and lonic Hydrogen-Bonding Interactions. J. Phys. Chem. A 117, 12590-12600, (2013).

3 Becke, A. D. Density-functional Thermochemistry. III. The Role of Exact Exchange. J. Chem. Phys. 1993, $98,5648$.

4 Lee, C.; Yang, W.; Parr, R. G. Development of the Colle-Salvetti Correlation-energy Formula into a Functional of the Electron Density. Phys. Rev. B 1988, 37, 785.

5 Grimme, S. Semiempirical GGA-Type Density Functional Constructed with a Long-Range Dispersion Correction. J. Comput. Chem. 2006, 27, 1787.

6 Marenich, A. V., Cramer, C. J. \& Truhlar, D. G. Universal Solvation Model Based on Solute Electron Density and on a Continuum Model of the Solvent Defined by the Bulk Dielectric Constant and Atomic Surface Tensions. J. Phys. Chem. B 113, 6378-6396, (2009).

7Tomasi, J., Mennucci, B. \& Cancès, E. The IEF version of the PCM solvation method: an overview of a new method addressed to study molecular solutes at the $\mathrm{QM}$ ab initio level. J. Mol. Struct.: THEOCHEM 464, 211-226, (1999).

8 Experiment results in dichloroethane and toluene were quite similar. Since most uses of 2-3 are documented in toluene, the computational work used toluene as well.

9 Schrödinger Release 2018-4: Jaguar, Schrödinger, LLC, New York, NY, 2018.

10 Legault, C. Y. CYLview, version 1.0b; Universitéde Sherbrooke: Quebec, Canada, 2009; http://www.cylview.org.

11 Zhurko, G.; Zhurko, D. ChemCraft, Version 1.8 http://www.chemcraftprog.com.

12 Storer, J. W., Giesen, D. J., Cramer, C. J. \& Truhlar, D. G. Class IV charge models: A new semiempirical approach in quantum chemistry. J. Comput.-Aided Mol. Des. 9, 87-110, (1995).

13 Schrödinger Release 2019-2: MacroModel, Schrödinger, LLC, New York, NY, 2019. 
14 (a) Bader, R. F. W. 1990 In Atoms in Molecules: A Quantum Theory (Oxford: Clarendon Press); (b) Bader, R. F. W. Atoms in Molecules.Acc. Chem. Res. 18 9-15, (1985); (c) Bader, R. F. W. A Quantum Theory of Molecular Structure and Its Applications. Chem. Rev. 91, 893-928, (1991).

15 Ziółkowski, M., Grabowski, S. J. \& Leszczynski, J. Cooperativity in Hydrogen-Bonded Interactions: Ab Initio and "Atoms in Molecules" Analyses. J. Phys. Chem. A 110, 6514-6521, (2006).

16 (a) Grabowski, S. J. What is the Covalency of Hydrogen Bonding? Chem. Rev. 111, 2597-2625, (2011); (b) Grabowski, S. J. QTAIM Characteristics of Halogen Bond and Related Interactions. J. Phys. Chem. A 116, 1838-1845, (2012).

17 Matta, C. F. 2006 In Hydrogen Bonding - New Insights S J Grabowski (Ed.) (New York: Springer) Ch. 9 